\title{
SCULPTING DESIRED TOPOGRAPHIES USING ABRASIVE JET MICRO-MACHINING
}

\author{
by \\ Mohammadreza Sookhaklari \\ BSc, Mechanical Engineering, Iran Shiraz University, 2008 \\ MSc, Mechanical Engineering, Shiraz University, 2011
}

\author{
A dissertation \\ presented to Ryerson University \\ in partial fulfillment of the \\ requirement for the degree of \\ Doctor of Philosophy \\ in the program of \\ Mechanical Engineering \\ Toronto, Ontario, Canada, 2016 \\ (C) Mohammadreza Sookhaklari, 2016
}




\section{AUTHOR'S DECLARATION}

I hereby declare that I am the sole author of this dissertation. This is a true copy of the dissertation, including any required final revisions, as accepted by my examiners.

I authorize Ryerson University to lend this dissertation to other institutions or individuals for the purpose of scholarly research.

I further authorize Ryerson University to reproduce this dissertation by photocopying or by other means, in total or in part, at the request of other institutions or individuals for the purpose of scholarly research.

I understand that my dissertation may be made electronically available to the public. 


\title{
SCULPTING DESIRED TOPOGRAPHIES \\ USING ABRASIVE JET MICRO-MACHINING
}

\author{
Mohammadreza Sookhaklari \\ Doctor of Philosophy, 2016 \\ Mechanical Engineering \\ Ryerson University
}

\begin{abstract}
Abrasive jet micromachining (AJM) uses a jet of high-speed particles to erode a wide variety of materials. Given a set of process parameters, surface evolution models capable of predicting the shape of straight, constant-depth channels in a wide variety of materials are wellestablished. This dissertation presents novel methods for solving the unaddressed more challenging and industrially relevant inverse problem of determining the process parameters required to machine a particular user-specified feature topography.

Since the air driven jet used in AJM is divergent, the edges of the desired features are usually defined using a mask which is attached to the surface of the target material. This dissertation presents alternate techniques using stationary or moving shadow masks that can be moved over the surface and maskless techniques, in order to allow direct writing of desired features on the surface. A mathematical framework is then introduced to determine the direct writing source velocity function and path required to create a desired shallow topography. It is also shown how the methodology can be used with existing surface evolution models to predict the feature shape at any depth. The methodologies are demonstrated to work well for the AJM of constant depth micro-channels with user-specified cross-sectional shape, gradient etched micro-channels with specified texture along their length, and pockets with texture in two perpendicular directions.

Finally, a new technique is introduced that utilizes a rotating patterned mask in order to control the AJM erosive footprint size and shape. Models for predicting the rotating mask pattern required to create virtually any desired footprint are presented, and experimentally verified for symmetric and asymmetric W-shaped, trapezoidal and wedge shaped footprints.
\end{abstract}




\section{Acknowledgements}

I would like to sincerely thank:

* Prof. Papini for his support, patience and motivation through my Ph.D. study. It was a great opportunity having him as an advisor and a mentor for my Ph.D. study.

* The Natural Sciences and Engineering Research Council of Canada (NSERC) and the Canada Research Chairs program for financial support.

* Thanks are also due to Prof. Spelt in the department of Mechanical Engineering at the University of Toronto and Prof. G. Papini in the department of Physics at the University of Regina for useful discussions.

My father Ghodratollah, mother Parvaneh and siblings Sanaz, Roxana and Amin for all their support and love. 


\section{Dedication}

To my parents for all their love and support. I appreciated everything they have done for me and all the sacrifices they have made. Reaching this far was not possible without them. 


\section{Table of contents}

AUTHOR'S DECLARATION .................................................................................. ii

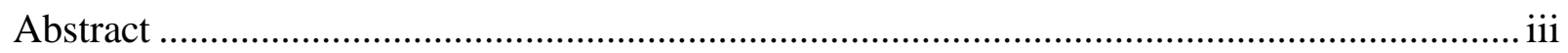

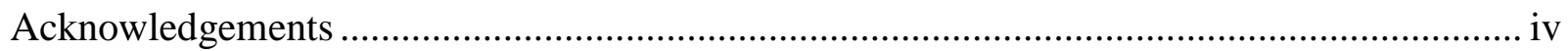

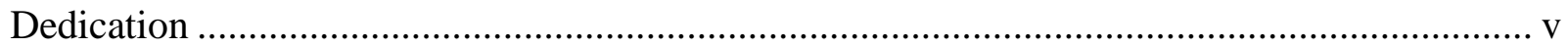

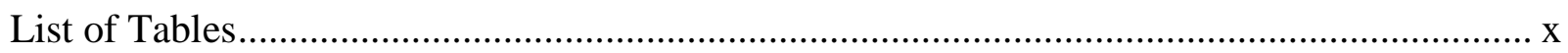

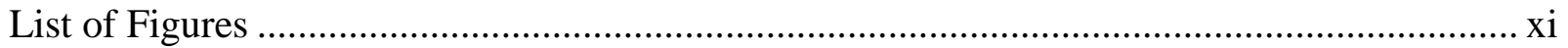

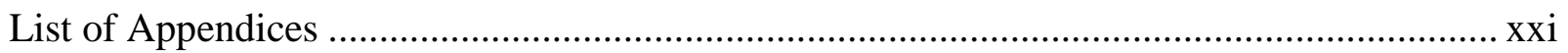

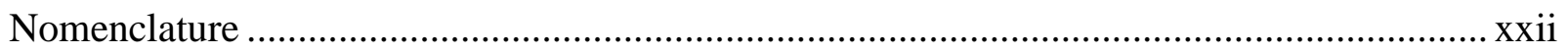

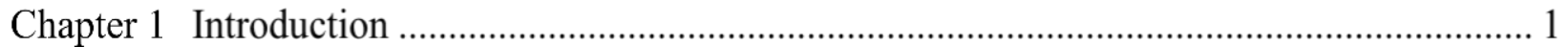

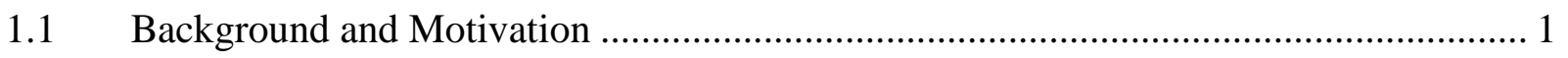

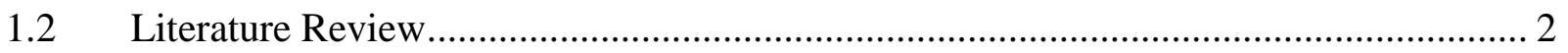

1.2.1 Solid particle erosion mechanisms......................................................... 2

1.2.2 Mask materials used in abrasive jet micro-machining ....................................... 6

1.2.3 Modeling of feature shape resulting from AJM ................................................. 8

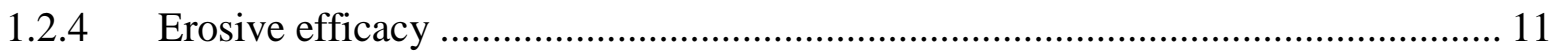

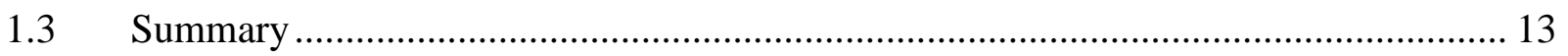

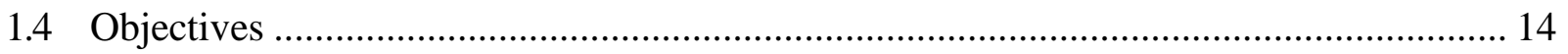

Chapter 2 Implementation of a shadow mask for direct writing in abrasive jet micro-machining

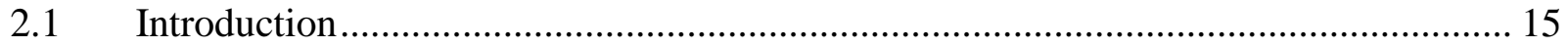

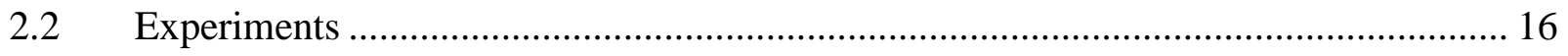

2.2.1 Shadow Mask Apparatus ............................................................................... 16

2.2.2 Measurement of channel cross-section and frosted region ................................ 21

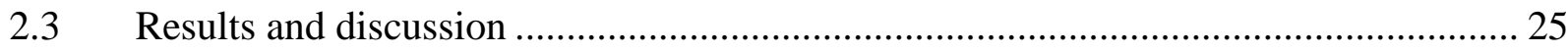

2.3.1 Clamped vs. shadow mask ....................................................................... 25

2.3.2 Effect of nozzle standoff distance ................................................................. 26

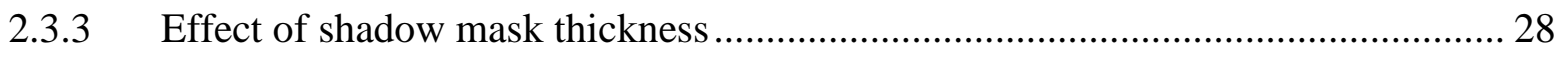

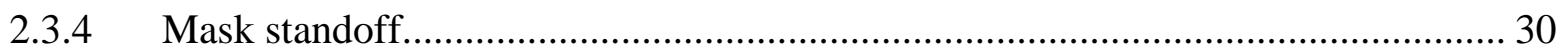




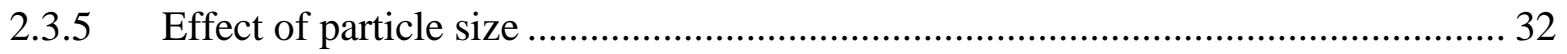

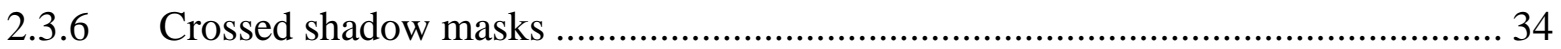

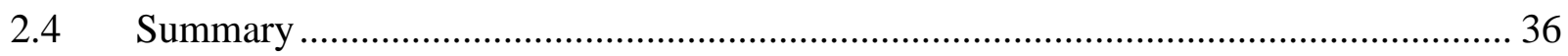

Chapter 3 Control of micro-channel cross-sectional shape in the abrasive jet micro-machining

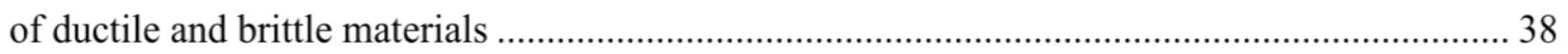

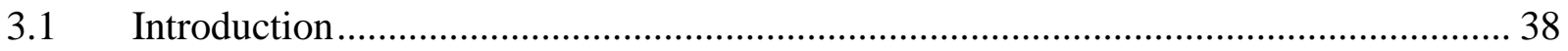

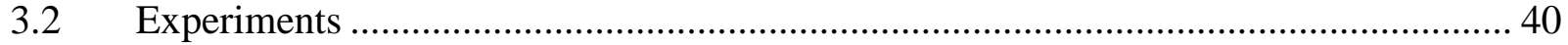

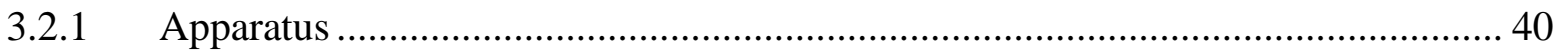

3.2.2 Measurement of shadow mask erosion ............................................................. 41

3.2.3 Micro-machining experiments using oscillating target.......................................... 43

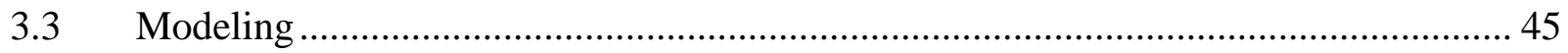

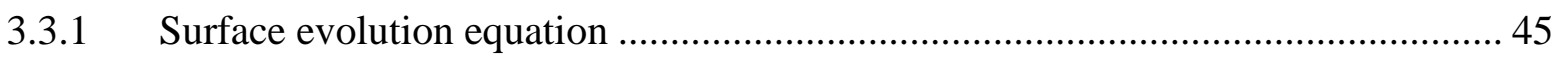

3.3.2 Determination of erosive efficacy ......................................................................... 47

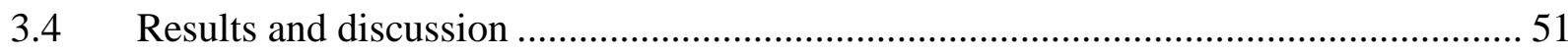

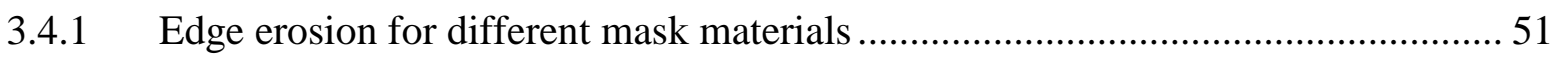

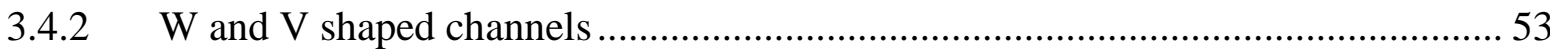

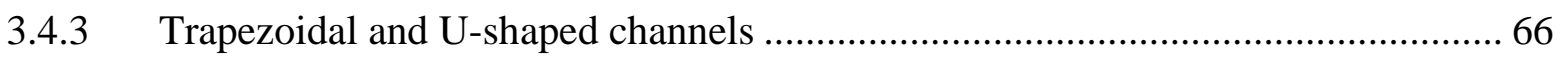

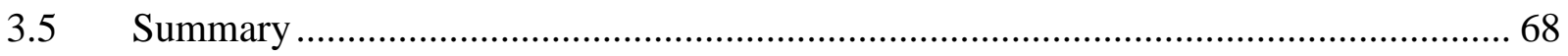

Chapter 4 Inverse methods to gradient etch three-dimensional features with prescribed topographies using abrasive jet micro-machining: Modelling....................................................... 70

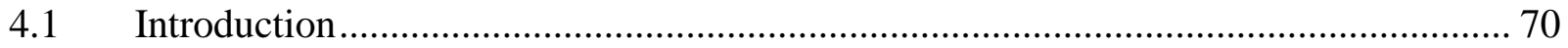

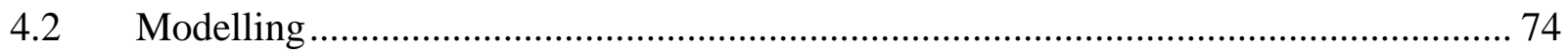

4.2.1 Total first pass erosive efficacy delivered by a moving source .............................. 74

4.2.2 Source velocity required to obtain a desired initial topography .............................. 76

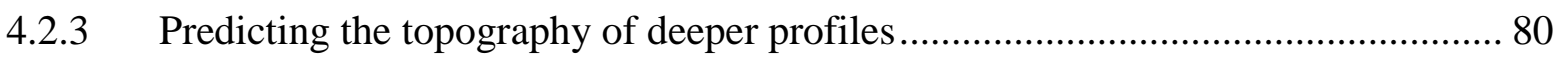

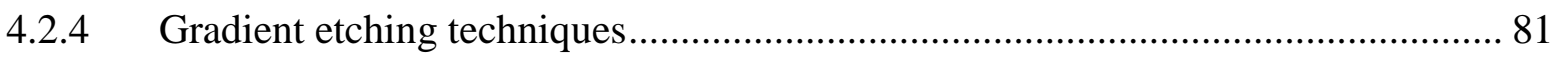

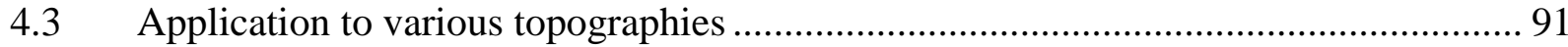

4.3.1 Channels with desired texture along their length................................................... 91

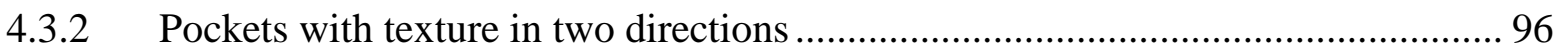

4.3.3 Channels with prescribed cross sectional shape …….............................................. 99

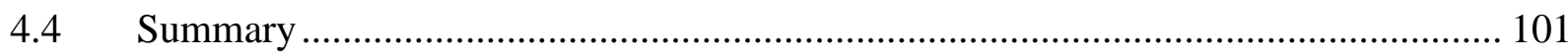


Chapter 5 Experimental verification of inverse methods to gradient etch three-dimensional features with prescribed topographies

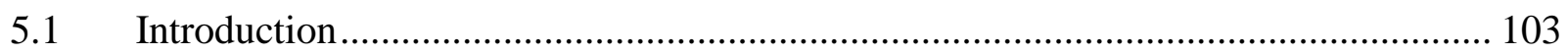

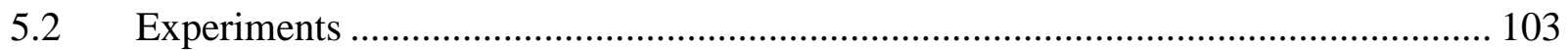

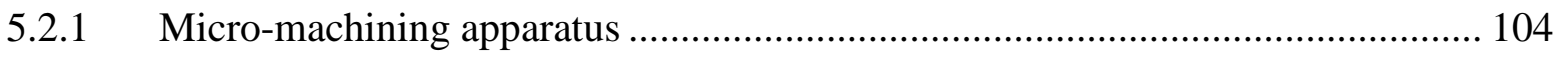

5.2.2 Effect of machining step size ..................................................................... 105

5.2.3 Micro-machining channels with desired texture along their length....................... 106

5.2.4 Pockets with texture in two directions ................................................................. 106

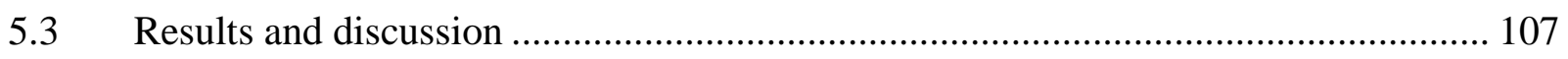

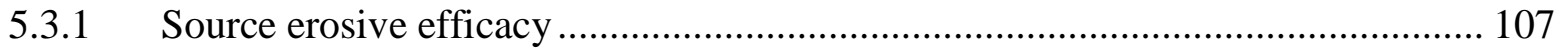

5.3.2 Micro-machining of channels with desired texture along their length .................. 107

5.3.3 Pockets with texture in two directions ........................................................... 116

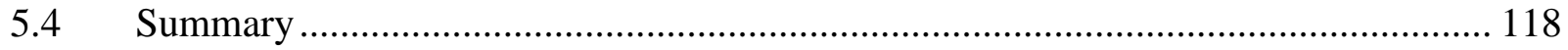

Chapter 6 A rotating mask system for sculpting of three-dimensional features using abrasive jet

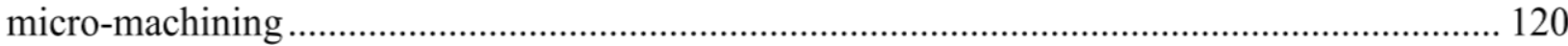

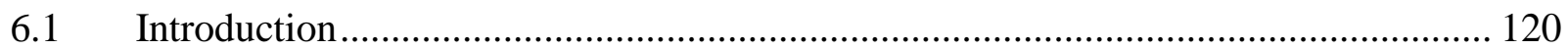

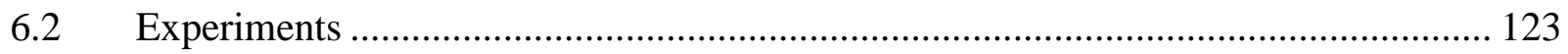

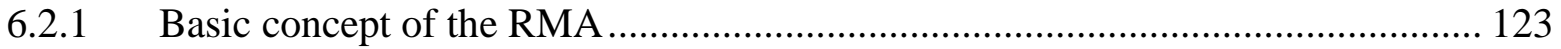

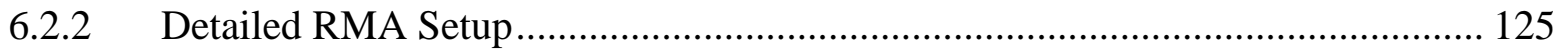

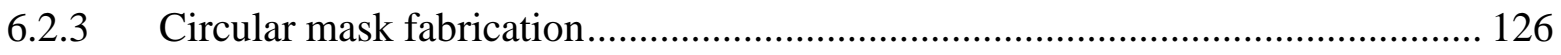

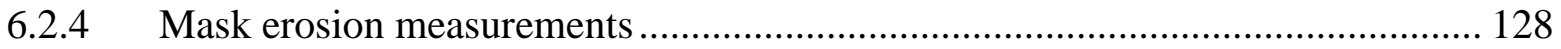

6.2.5 Effect of mask standoff on pattern transfer........................................................ 129

6.2.6 Effect of distance between circles on mask ....................................................... 130

6.2.7 Effect of disk rotational speed ...................................................................... 131

6.2.8 Machining of channels of various cross-sectional shapes using the RMA........... 132

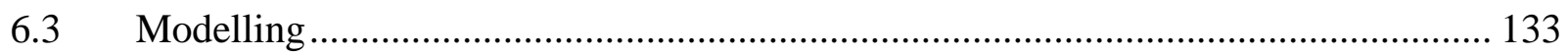

6.3.1 Functional form of the erosive efficacy through a series of holes in a given base arc

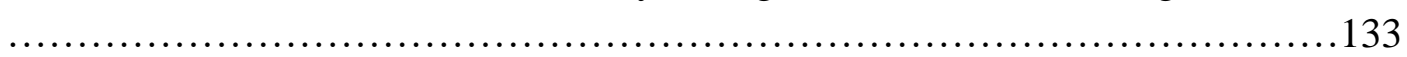

6.3.2 Net erosive efficacy through a pattern of holes on different base arcs ................. 134

6.3.3 Determination of pattern needed to produce a desired erosive efficacy footprint 136

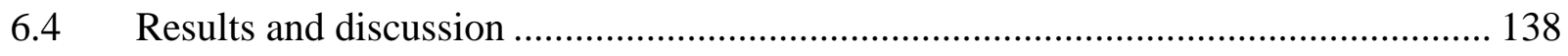


6.4.1 Mask erosion ..................................................................................... 138

6.4.2 Effect of mask standoff on pattern transfer................................................ 139

6.4.3 Effect of distance between circles on mask ................................................... 140

6.4.4 Machining of channels of various cross-sectional shapes ............................... 142

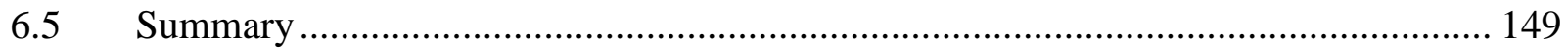

Chapter 7 Summary and Conclusions …....................................................................... 151

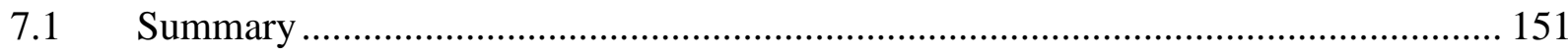

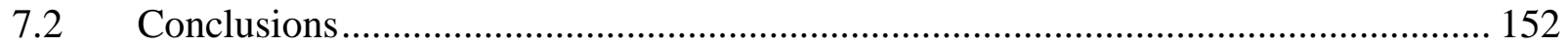

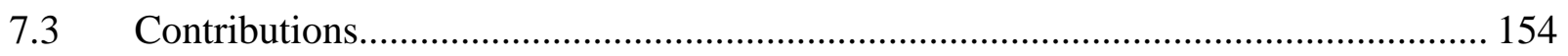

7.4 Recommendations for future work ................................................................. 154

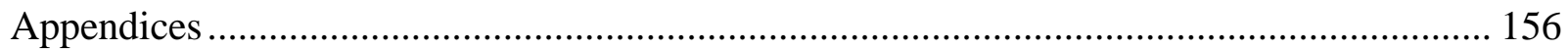

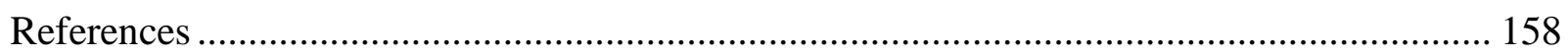




\section{List of Tables}

Table 2-1 Summary of conditions used in each experiment................................................. 20

Table 3-1 Specimen Rockwell hardness ......................................................................... 42

Table 3-2 Process parameters used in experiments ........................................................... 44

Table 4-1 Examples of desired non-dimensional microchannel textures and velocity functions necessary to produce them using method of Section 4.2.2.1. Gaussian source erosive efficacy with

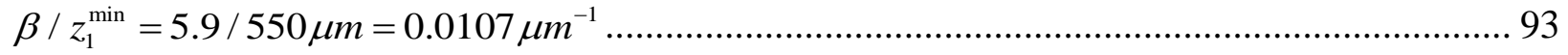




\section{List of Figures}

Figure 1-1 Schematic of a typical abrasive jet apparatus. ................................................. 2

Figure 1-2 Schematic of crack formation during solid particle impact [26]............................ 6

Figure 1-3 Schematic of coordinates used for masked channel cross-sectional profiles [37]....... 8

Figure 1-4 Two spherical particles approaching the mask opening [43] ............................... 12

Figure 2-1 Shadow mask apparatus. Nozzle standoff $(N S)$ and mask standoff $(M S)$ were defined relative to the top of the mask. Only two of three springs shown. ............................................ 18

Figure 2-2 Relative orientation of nozzle and shadow mask........................................... 18

Figure 2-3 Jet aperture for direct writing of curved paths made using two sets of crossed shadow masks. 19

Figure 2-4 Location of edge of frosted area (dashed line) and a comparison of channel width based on (i) the slope method (dashed dotted line) and (ii) the channel crater size method (dotted line).

Figure 2-5 Top view of glass channel blasted at $200 \mathrm{kPa}, 20 \mathrm{~mm}$ nozzle standoff, with a contacting crossed shadow mask having a square opening $260 \mu \mathrm{m}$ on a side. The $30 \mu \mathrm{m}$ wide frosted regions are indicated. 24

Figure 2-6 Segmented image of Figure 2-5 showing the frosted regions in white. 24

Figure 2-7 Cross-sectional profiles of channels machined using various number of passes and the shadow mask of Figure 2-2 in contact with the surface (solid and dashed lines) or a clamped mask (symbols). Dimensions normalized using the mask opening, $W=293 \mu \mathrm{m}$. Only half of each symmetric profile is shown. Mask thickness of $800 \mu \mathrm{m}, N S=20 \mathrm{~mm}$. 26

Figure 2-8 Comparison of typical channel profiles obtained after 4 passes of the shadow mask of Figure 2-2 at three nozzle standoff distances (Figure 2-1). Depth and width normalized by mask opening $W=270 \mu \mathrm{m}$. 27 
Figure 2-9 Average channel depth (squares) and width (circles) at three nozzle standoffs. Scatter bars representing \pm 1 standard deviation for 9 measurements (3 measurements on each of 3 specimens) were small enough to fit within the symbols.

Figure 2-10 Average frosted width at three nozzle standoffs (Figure 2-1). The scatter bars represent \pm 1 standard deviation for 9 measurements ( 3 measurements on each of 3 specimens). ............ 28

Figure 2-11 Effect of mask thickness on typical channel profiles obtained after 4 passes of the shadow mask of Figure 2-2. Channel depth and width normalized by mask opening $W=270 \mu \mathrm{m}$.

Figure 2-12 Average channel depth, width and frosted width for mask thicknesses of 0.8 and 1.6 $\mathrm{mm}$. The scatter bars represent \pm 1 standard deviation for 9 measurements ( 3 measurements on each of 3 specimens).

Figure 2-13 A particle striking the edge of a thin mask (a) is more likely to strike the surface, while that striking a thick mask (b) collides with the opposing mask. 30

Figure 2-14 Typical channel profiles obtained after 4 passes of the shadow mask of Figure 2-2 using mask-to-surface standoff distances of 0 and $2 \mathrm{~mm}$ (Figure 2-1). Channel depth and width normalized by mask opening $W=270 \mu \mathrm{m}$. 31

Figure 2-15 Average depth, width and frosted width for mask standoffs of 0 and $2 \mathrm{~mm}$. The scatter bars represent \pm 1 standard deviation for 9 measurements ( 3 measurements on each of 3 specimens).

Figure 2-16 Channel profiles obtained after 4 passes of the shadow mask of Figure 2-2 using 10 and $25 \mu \mathrm{m}$ particles. Channel depth and width normalized by mask opening $W=290 \mu \mathrm{m}$. 33 Figure 2-17 Average channel depth, width and frosted width for particle sizes of 10 and $25 \mu \mathrm{m}$. The scatter bars represent \pm 1 standard deviation for 9 measurements ( 3 measurements on each of 3 specimens). 33

Figure 2-18 Profiles of channels made after 4 passes of the crossed shadow mask (Figure 2-3) and the regular shadow mask (Figure 2-2). Channel depth and width normalized by mask opening $W=290 \mu \mathrm{m}$ 35 
Figure 2-19 Footprints of the erodent jets produced using the crossed shadow mask (left) and the regular mask (right). For the crossed mask, the slot of the top set of masks was in the vertical direction.

Figure 2-20 Ryerson University logo machined in glass using direct writing technique with crossed shadow masks. 36

Figure 3-1 Conceptual schematic of shadow clamp of Chapter 2 41

Figure 3-2 Schematic of an oscillating pass. $L$ is the lead, which when divided by the scan speed, gave the frequency. The location of the center of the source relative to the oscillating target is described by $x_{0}(t)$ 44

Figure 3-3 Schematic of coordinates used for masked channel cross-sectional profiles. 45

Figure 3-4 Footprint left by the nozzle. 47

Figure 3-5 Coordinate system for a channel resulting from a stationary erosive efficacy source $E_{L}$, of length $L_{c}$ 48

Figure 3-6 Erosion rate for different mask materials with error bars representing one standard deviation 52

Figure 3-7 Profile of edges after erosion tests. Edge of samples prior to erosion is indicated by the dashed line. 53

Figure 3-8 Predicted normalized erosive efficacy using the model of Section 3.3.2 (solid line, Eq. (3.10) and symbols, Eq. (3.18)). Amplitude $A=400 \mu \mathrm{m}, \omega=15 \mathrm{~Hz}$. Widths, $x$, normalized by dividing by width of oscillating channel. Erosive efficacies, $E$, normalized by dividing by nonoscillating pass (dashed line) centerline erosive efficacy machined at $v_{s}=1 \mathrm{~mm} / \mathrm{s}$ using maskless technique. 54

Figure 3-9 Effect of amplitude on measured (symbols) and predicted (lines) micro-channel profiles made using oscillation on glass. A shadow mask opening of $156 \mu \mathrm{m}, \omega=10 \mathrm{~Hz}, v_{s}=2 \mathrm{~mm} / \mathrm{s}$, and 2 passes were used. The circles for $\mathrm{A}=0$ represent the non-oscillating pass. 56 
Figure 3-10 Effect of amplitude on channel shape for $A=100,200,300$ and $400 \mu \mathrm{m}$ and $\omega=15$ Hz. Width $\mathrm{x}$, and depth $z$, normalized by dividing by $2 A+W_{f}$, and $E(x=0)$ of widest channel, respectively. 56

Figure 3-11 Schematic of foot prints of nozzle at the beginning and at half a period time span; the dashed line shows the path of oscillation. 57

Figure 3-12 A typical "W" shape profile machined in glass made by oscillation of the target below the shadow mask setup. Experimental conditions; mask opening $=156 \mu \mathrm{m}, A=250 \mu \mathrm{m}, v_{s}=2 \mathrm{~mm} / \mathrm{s}$ and $\omega=9 \mathrm{~Hz}$. The darker horizontal line a distance behind the front edge is an artifact of the SEM

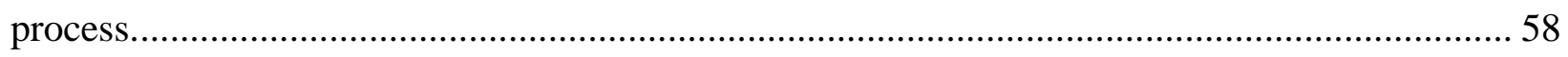

Figure 3-13 Effect of mask opening on non-oscillating channels (two passes machined at $v_{s}=1$ $\mathrm{mm} / \mathrm{s})$. 59

Figure 3-14 Predicted (lines) and measured (symbols) micro-channel profiles machined using different mask openings for 4 passes at $v_{s}=1 \mathrm{~mm} / \mathrm{s}$ with target oscillating at $\omega=15 \mathrm{~Hz}$, and $A=$ 250. Symbols are the experimental results and solid lines are the results from the model. 59

Figure 3-15 Comparison of measured (symbols) and predicted (lines) micro-channel crosssectional profiles machined using oscillation with $A=320 \mu \mathrm{m}, v_{s}=1 \mathrm{~mm} / \mathrm{s}$ and $\omega=15 \mathrm{~Hz}$ in glass using the shadow mask with opening of $410 \mu \mathrm{m}$. The depth and width are normalized by $2 A+W_{f}=$ $1237 \mu \mathrm{m}$. Number of passes is shown on each line. (a) shallow channels; (b) deeper channels... 61

Figure 3-16 Edge frosting for a typical W-shaped channel. Solid lines show the edge of the channel and the dashed lines show the edge of the frosted area.

Figure 3-17 Comparison of predicted (symbols) and measured (lines) cross-sectional profiles of channels machined at amplitude of $320 \mu \mathrm{m}, v_{s}=1 \mathrm{~mm} / \mathrm{s}$ and $\omega=15 \mathrm{~Hz}$ in PMMA using maskless technique with various number of passes from experiments (symbols) and probability model (lines). The depth and width are normalized by $2 A+W_{f}=1081 \mu \mathrm{m}$. Number of passes is shown on each line. (a) shallow channels; (b) deeper channels; (c) very deep channels. 64

Figure 3-18 A typical W shaped channel machined in PMMA at amplitude of $250 \mu \mathrm{m}, v_{s}=1 \mathrm{~mm} / \mathrm{s}$ and $\omega=15 \mathrm{~Hz}$ in PMMA using maskless technique. 65

Figure 3-19 An example of small W shaped channel machined using shadow mask in PMMA. 65 xiv 
Figure 3-20 Predicted (lines) and measured (symbols) typical trapezoidal channel cross sections machined in glass by using oscillation method followed by additional passes at the center. Oscillation was at $A=300 \mu \mathrm{m}$ and $\omega=15 \mathrm{~Hz}$, with one (circles) and two (squares) passes at $v_{s}=1$ $\mathrm{mm} / \mathrm{s}$, followed by 1 (circles) and 2 extra non-oscillating passes (squares), in the center with $v_{s}=$ $3.5 \mathrm{~mm} / \mathrm{s}$

Figure 3-21 SEM images of trapezoidal and U shaped channels machined in (a) glass and (b) PMMA.

Figure 3-22 A triple via channel machined in glass by two oscillating passes $(A=300 \mu \mathrm{m}, \omega=15$ $\mathrm{Hz}$ and $v_{s}=1 \mathrm{~mm} / \mathrm{s}$ ) followed by three middle non-oscillating passes with scan speed of $3.5 \mathrm{~mm} / \mathrm{s}$.

Figure 4-1 Schematic showing moving AJM source whose centerline is instantaneously located at $s=s_{0}$ 75

Figure 4-2 Non-dimensionalized erosive efficacy through a $490 \mu \mathrm{m}$ shadow mask opening (circles), and a maskless circular source of $460 \mu \mathrm{m}$ diameter at $100 \mu \mathrm{m}$ standoff (dashed line). The solid line shows the least squares best fit curve of Eq. (4.6) with $\beta=5.9$, assuming that the length dimensions are normalized by the width of the footprint $(550 \mu \mathrm{m})$, and the erosive efficacy by its value at $s^{*}=0$. 77

Figure 4-3 Source moving in $y$-direction at velocity $v_{y}(y)$ to create a channel with a varying depth. 82

Figure 4-4 Prediction of source velocity and path to obtain desired pocket texture. (a) Desired double sinusoidal texture on a pocket having wavelength and amplitude of, respectively, 2 and 1, and 100/3 and 1, in the $x$ and $y$ directions, respectively. (b) Centerline profiles of three adjacent strips. (c) Predicted profile of one strip. (d) Resultant texture from three adjacent strips. .85

Figure 4-5 Schematic of two adjacent strips showing strip width $\left(w_{s}\right)$, width of erosive efficacy $\left(w_{e}\right)$ and width of overlapped area $\left(w_{o}\right)$....... 86

Figure 4-6 Schematic of foot prints of nozzle at the beginning and at half a period time span; the dashed line shows the path of oscillation. 87 
Figure 4-7 Expected shape of feature resulting from oscillation of a Gaussian source in range $1<x^{*}<1$ 89

Figure 4-8 A Gaussian source (dashed line) moving in the $x$-direction with velocity $v_{s}^{*}+C$ to create the desired feature (solid line) centered at $x^{*}=0$ with bounds at $x^{*}=1+W / 2 A$ and $-1-W / 2 A$. $W$ is the width of the source. 90

Figure 4-9 Predicted profiles using finite Gaussian (solid line) and point source (dashed line) solutions for a desired (circles, wavelength $=452 \mu \mathrm{m}$, amplitude $=35 \mu \mathrm{m}$ and $\beta=0.4$ ) sinusoidal texture of lower wavelength. It has been assumed that the point source strength, $K=z_{c} v_{c}=1 . .95$

Figure 4-10 Predicted velocities to machine the desired profile of Figure 4-9 using Gaussian finite (solid line) and point source (dashed line) solutions to inverse problem. It has been assumed that the point source strength, $K=z_{c} v_{c}=1$. 96

Figure 4-11 Desired patterns of $z^{*}\left(y^{*}\right)=11+10 \cos \left(6 \pi y^{*} / 100\right)$ (squares), $z^{*}\left(y^{*}\right)=11-y^{*} / 10$ (line) and $z^{*}\left(y^{*}\right)=1+y^{*} / 10$ (dashed line) that change every $\Delta x^{*}=0.75$. 97

Figure 4-12 Velocity functions required to machine the profiles of Figure 4-11 (symbols match with the ones in Figure 4-11). 98

Figure 4-13 Predicted pocket with desire texture of Figure 4-11 using a Gaussian foot print with $\beta / z_{1}^{\min }=3.89 / 50 \mu m=0.0778 \mu m^{-1}$ with strip width $w_{s}=0.75 w_{e}$ 98 Figure 4-14 Predicted texture for strip to efficacy width ratios $w_{s} / w_{e}$ of (a) 0.5 , (b) 0.4 (c) 0.3 and (d) 0.2. As the offset becomes smaller, the pattern deviates from the desired one. 99 Figure 4-15 Desired W-shaped channel cross section with $n=1 / 2, D=0.9$, and $C_{2}=0.5$ (dashed line) and that predicted to be machined using Gaussian source $\left(\beta / A=0.0085 \mu m^{-1}\right)$ with $v_{s}{ }^{*}=$ $0.834 \sqrt{1-x_{0}^{* 2}}$ (line) 100

Figure 4-16 The predicted required velocity function to machine the $\mathrm{W}$ - shaped channel with $\mathrm{n}=1 / 2$ and $\mathrm{D}=0.9$ (dashed line) in Figure 4-15. Solid line represents $v_{s}^{*}=0.834 \sqrt{1-x_{0}^{* 2}}$ (line). 100 
Figure 5-1 Erosive efficacies of shadow mask and maskless nozzle, normalized in width, $W$, by $411 \mu \mathrm{m}$ and $515 \mu \mathrm{m}$ respectively, and in depth by the value of erosive efficacy at $x=0$. For the shadow mask, $E^{*}\left(x^{*}\right)=e^{-\left(\beta x^{*}\right)^{2}}$ with $x^{*}=\frac{x}{411 \mu m}$ and $\beta=3.873$.

Figure 5-2 (a) Desired, predicted, and measured channel centerline profiles for a desired linear depth of $z_{1}(y)=51+128 y / 5000 \mu \mathrm{m}$; (b) predicted velocity function required to machine the desired profile. A maskless source was used with an equivalent point source strength of $K=z_{c} v_{c}=128 \times 10^{3} \mu m^{2} / s$ 109

Figure 5-3 (a) Measured and (b) predicted 3D profile for channel of Figure 5-1. Dimensions in $\mu \mathrm{m}$. 110

Figure 5-4 Desired, predicted, and measured channel centerline profiles for a desired parabolic depth of $z_{1}(y)=-490(y / 5000)^{2}+490 y / 5000+49 \mu \mathrm{m}$; (b) predicted velocity function required to machine the desired profile. A maskless source was used with an equivalent point source strength of $K=z_{c} v_{c}=123 \times 10^{3} \mu m^{2} / s$.

Figure 5-5 (a) Measured and (b) predicted 3D profile for channel of Figure 5-4. Dimensions in $\mu \mathrm{m}$.

Figure 5-6 Desired, predicted, and measured channel centerline profiles for a desired sinusoidal depth of $z_{1}(y)=140+100 \cos (4 \pi y / 5000) \mu \mathrm{m}$; (b) predicted velocity function required to machine the desired profile. A maskless source was used with an equivalent point source strength of $K=z_{c} v_{c}=100 \times 10^{3} \mu m^{2} / s$. 113

Figure 5-7 (a) Measured and (b) predicted 3D profile for channel of Figure 5-6. Dimensions in $\mu \mathrm{m}$.

Figure 5-8 SEM image (a) of a channel machined using the maskless technique with depth varying sinusoidally for 3 periods over a $5 \mathrm{~mm}$ length; (b) shows a magnified view of the square region highlighted in (a). 
Figure 5-9 Velocity function predicted by finite Gaussian source solution and point source solution. 115

Figure 5-10 Measured profiles resulting from machining with velocity function predicted by finite Gaussian source solution and point source solution, compared to desired profile with wavelength of $5000 / 3 \mu \mathrm{m}$. The Gaussian footprint of the source was approximately $1.7 \mathrm{~mm}$ wide $(\beta=0.11)$.

Figure 5-11 (a) Desired 3D pocket texture and (b) texture resulting from machining using the predicted velocities in Figure 5-12. A maskless source was used with an equivalent point source strength of $K=z_{c} v_{c}=75 \times 10^{3} \mu \mathrm{m}^{2} / \mathrm{s}$. Dimensions are in $\mu \mathrm{m}$. 116

Figure 5-12 Velocity functions required to machine the desired 3D pocket texture in Figure 5-11 (a). 116

Figure 5-13 Predicted (right side) versus experimental (left side) textures for offset of (a) 300 and (b) $400 \mu \mathrm{m}$. As the offset becomes smaller, the pattern deviates from the desired one in Figure 511(a). Dimensions are in $\mu \mathrm{m}$.

Figure 5-14 Images of textures machined using maskless technique with (a) $\Delta x=500 \mu \mathrm{m}$ and (b) $\Delta x=400 \mu \mathrm{m}$. The camera was tilted to capture the patterns. 118

Figure 6-1 (a) Side (section g-g) and (b) top views of conceptual schematic of RMA disk, showing elastomeric mask suspended across disk slot. $r_{0 i}$ are the radial positions of base arcs on which holes were cured in the mask material. Crosshatched region is the open slot in the disk over which the patterns in the mask are suspended. Schematic is not to scale. 124

Figure 6-2 Schematic of rotating disk mechanism. 126

Figure 6-3 (a) Schematic of a mask showing the base arcs and alignment features, and (b) The mask master printed on the transparency. 127

Figure 6-4 Fixture used for mask standoff experiments. 130 Figure 6-5 Schematic of hole placement on the disk and foot print of nozzle. Figure is not to scale. 131

Figure 6-6 Schematic of a particle passing through a hole in the mask. 132 xviii 
Figure 6-7 A non-dimensionalized channel $\left(x^{*}=x / 575\right.$ and $\left.E^{*}=z^{*}=z / 112\right)$ machined with RMA and a $300 \mu \mathrm{m}$ hole pattern with single base arc (circles). Solid line shows the best fit curve of Eq.(6.3) with $\beta=4.5$.

Figure 6-8 Schematic of (a) positive directions used for Eq. (6.4) and (b) "i" and "j”" numbering for a $60^{\circ}$ portion of a pattern. " $\mathrm{n}$ " is the $\mathrm{n}^{\text {th }}$ base arc and " $\mathrm{m}$ " is the $\mathrm{m}^{\text {th }}$ hole on that arc. 136 Figure 6-9 Mask durability for three configurations. Mass flow rate was $\sim 2.1 \mathrm{~g} / \mathrm{s}$ in all cases. The scatter bars represent \pm 1 standard deviation for 3 measurements. 139

Figure 6-10 Effect of mask standoff on pattern transfer. The scatter bars represent \pm 1 standard deviation for 5 repetitions 139

Figure 6-11 Three modes of failure in elastomeric mask. (a) Abrupt tear between two adjacent holes (bridge length=400 $\mu \mathrm{m}$ ). (b) Two holes became connected (bridge length=200 $\mu \mathrm{m}$ ). (c) failure of the mask material (bridge length $=600 \mu \mathrm{m}$ ). The scales on the figures are $300 \mu \mathrm{m} . . .140$ Figure 6-12 Average dose to failure from 3 repetition of the test for different bridge lengths of holes with diameter of (a) $300 \mu \mathrm{m}$ and (b) $600 \mu \mathrm{m}$. The scatter bars represent \pm 1 standard deviation for 3 measurements. 141

Figure 6-13 Comparison between side wall slopes of normalized channels (normalized by $872 \mu \mathrm{m}$ in width) machined with hole diameters of $300 \mu \mathrm{m}, 600 \mu \mathrm{m}$ and the combination of two with equal number of holes from each. Solid line shows the prediction of Eq. (6.4). 143

Figure 6-14 Profile of a symmetric W-shaped micro-channel. Two base arcs were used with radiuses of $20.3 \mathrm{~mm}$ and $20.7 \mathrm{~mm}$ each of which had 24 holes on it (mass flow rate=5.7 $\mathrm{g} / \mathrm{s}$ ). 144 Figure 6-15 Profile of a symmetric W-shaped micro-channel. Two base arcs were used with radiuses of $20.35 \mathrm{~mm}$ and $20.65 \mathrm{~mm}$ each of which had 24 holes on it (mass flow rate=5.7 $\mathrm{g} / \mathrm{s}$ ).

Figure 6-16 Profile of an asymmetric W-shaped micro-channel. Two base arcs were used with radiuses of $20.34 \mathrm{~mm}$ and $20.66 \mathrm{~mm}$ with 40 and 24 cured holes on them, respectively (mass flow rate $=5.6 \mathrm{~g} / \mathrm{s})$. 145 
Figure 6-17 Normalized profile of a trapezoidal W-shaped micro-channel $\left(\mathrm{z}^{*}=\mathrm{z} / 59.9\right.$ and $\left.x^{*}=x / 575\right)$. Five base arcs were used with radiuses of $19.95 \mathrm{~mm}, 20.19 \mathrm{~mm}, 20.5 \mathrm{~mm}, 20.78 \mathrm{~mm}$ and $21.05 \mathrm{~mm}$ each of which had 16 holes on it (mass flow rate=4.6 g/s)......................... 146

Figure 6-18 Profile of a trapezoidal W-shaped micro-channel. Five base arcs were used with radiuses of $19.95 \mathrm{~mm}, 20.19 \mathrm{~mm}, 20.5 \mathrm{~mm}, 20.78 \mathrm{~mm}$ and $21.05 \mathrm{~mm}$ with 20, 24, 28, 30 and 32 cured holes on them, respectively (mass flow rate $=4.2 \mathrm{~g} / \mathrm{s}$ ). 147

Figure 6-19 Profile of a wavy trapezoidal micro-channel. Three base arcs were used with radiuses of $19.95 \mathrm{~mm}, 20.5 \mathrm{~mm}$ and $21.05 \mathrm{~mm}$ each of which had 24 holes on it. 148 Figure 6-20 Profile of a wavy wedge shape micro-channel. Three base arcs were used with radiuses of $19.95 \mathrm{~mm}, 20.5 \mathrm{~mm}$ and $21.05 \mathrm{~mm}$ with 13,17 and 21 holes on them, respectively. 148

Figure 6-21 Higher aspect ratio narrow micro-channels machined using RMA with $200 \mu \mathrm{m}$ holes: (a) Trapezoidal channel using 3 base arcs with base arc radii of $20.4 \mathrm{~mm}, 20.5 \mathrm{~mm}$ and $20.6 \mathrm{~mm}$ (41, 14 and 41 holes on each arc). (b) Wedge shape microchannel using 5 base arcs with base arc radii of $20.1 \mathrm{~mm}, 20.3 \mathrm{~mm}, 20.5 \mathrm{~mm}, 20.7 \mathrm{~mm}$ and $20.9 \mathrm{~mm}(11,16,21,27$ and 32 holes on each arc). 149 


\section{List of Appendices}

Appendix A Coefficients of Fourier series for features in Table 4-1 ..................................... 156

Appendix B RMA pattern arrangement for different cross sectional profiles ........................ 157 


\section{Nomenclature}

$\begin{array}{ll}\alpha & \text { Impact angle } \\ \beta & \text { Focus coefficient } \\ \delta & \text { Dirac delta function } \\ \varepsilon & \text { Curvature-dependent smoothing term } \\ \rho & \text { Density of an average erodent particle } \\ \rho_{s} & \text { The density of the target material } \\ \varphi(x) & \text { Particle mass flux } \\ \omega & \text { Frequency of oscillation } \\ \omega_{\text {max }} & \text { Maximum RDA rotational speed to ensure enough time provided for } \\ & \text { particles to pass through the mask in a given rotation } \\ \omega_{\min } & \text { Minimum RDA rotational speed to ensure resulting channel free of undue } \\ & \text { waviness }\end{array}$

Depth of a hemispherical crater

A

Amplitude of oscillation

$A_{c}$

Cross-sectional area of material removed

C

Erosion rate constant

$C_{1}$

A constant defining the height of the Gaussian at the periphery of a channel

$C_{2}$

Desired non-dimensional centerline depth for W shape

d

Mask hole diameter in RMA

$d_{c}$

Bridge length between holes in RMA

$d_{m}$

Radial size of the footprint

$d_{0}$

Circumferential size of footprint 
$d_{p}$

$d_{r}$

$d_{i, j} *$

$D$

$\operatorname{erf}()$

$E(\alpha)$

$E 90$

$E_{c}(s)$

$E_{L}(s)$

$E_{N}(s, t)$

$E_{s}(s)$

$E_{s}(0)$

$E_{T}(s)$

ER

$F^{*}\left(s^{*}\right)$

$g(\alpha)$

$H$

$\bar{H}$

$H_{v}$

$i$

$i_{\max }$

j
Particle diameter

Radial distance between base arcs for RMA

Normalized depth

Maximum $x^{*}$ occurring of central peak for a W-shaped channel

Error function

Erosion rate as a function of the local impact angle

Erosion rate at normal incidence

Erosive efficacy delivered by the moving source with a constant velocity

$v_{c}$

Stationary erosive efficacy source

Nozzle erosive efficacy

Spatial distribution of source erosive efficacy

Value of source erosive efficacy over the centerline

Total erosive efficacy delivered by the moving source

Erosion rate

Desired non-dimensional efficacy footprint profile for RMA

Function describing the impact angle dependence of erosion rate

Heaviside function

The Vickers hardness of the target

The initial target hardness

Base arc number for RMA

The total number of base arcs for RMA

Hole number on a given base arc for RMA 


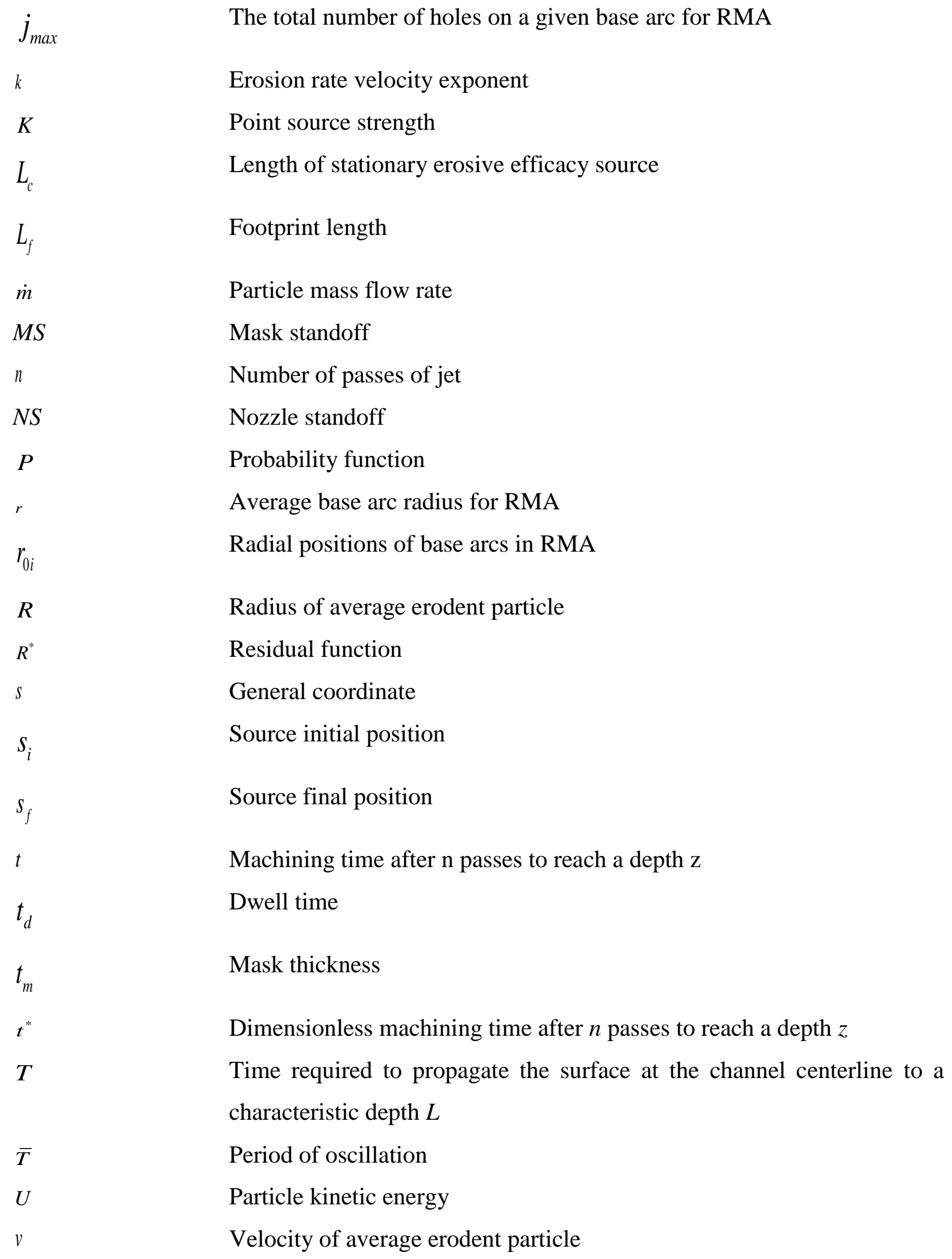

Erosion rate velocity exponent

Point source strength

Length of stationary erosive efficacy source

Footprint length

Particle mass flow rate

Mask standoff

Number of passes of jet

Nozzle standoff

Probability function

Average base arc radius for RMA

Radial positions of base arcs in RMA

Radius of average erodent particle

Residual function

General coordinate

Source initial position

Source final position

Machining time after $\mathrm{n}$ passes to reach a depth $\mathrm{z}$

Dwell time

Mask thickness

Dimensionless machining time after $n$ passes to reach a depth $z$

Time required to propagate the surface at the channel centerline to a characteristic depth $L$

Period of oscillation

Particle kinetic energy

Velocity of average erodent particle 


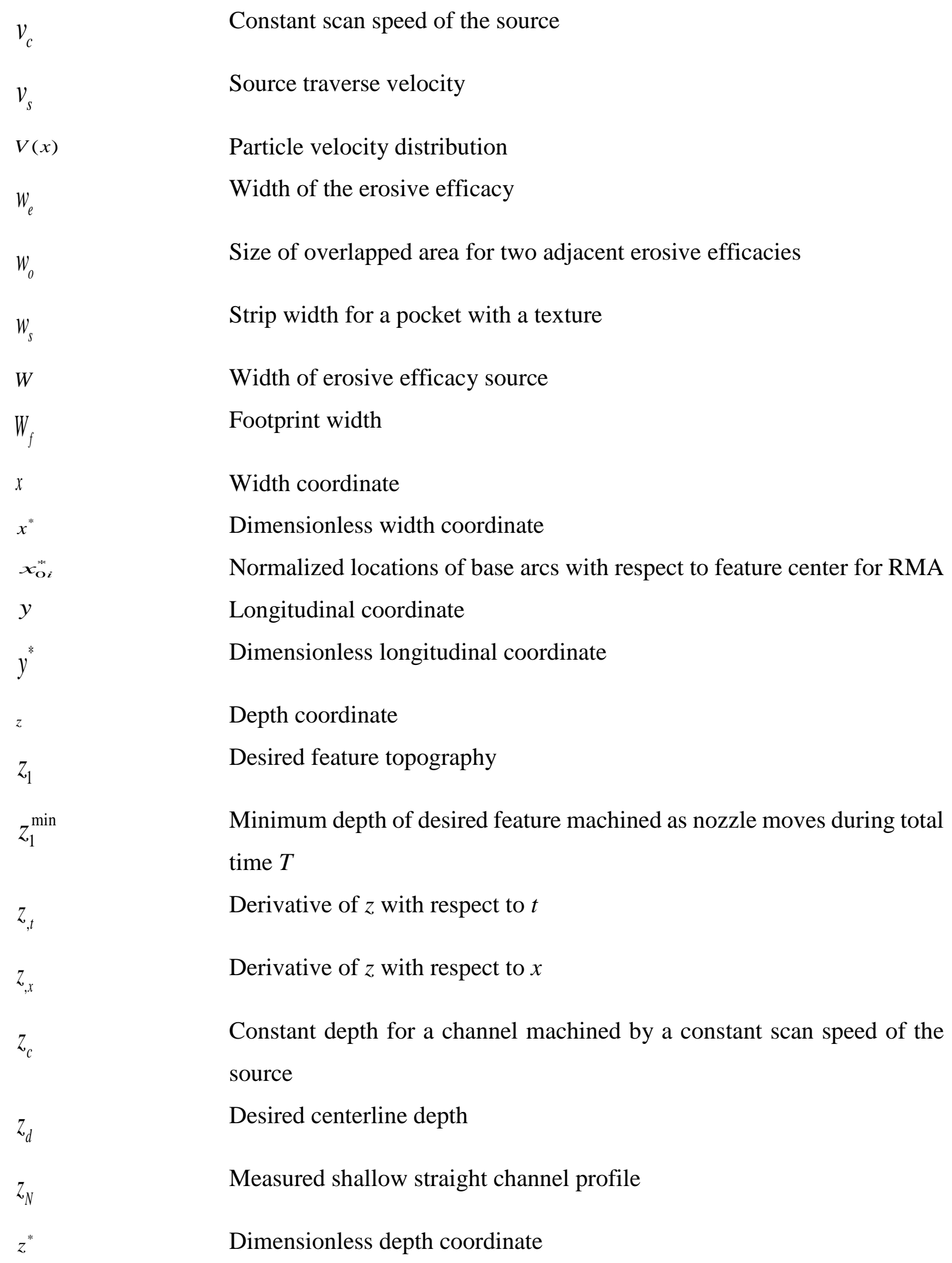

Source traverse velocity

Particle velocity distribution

Width of the erosive efficacy

Size of overlapped area for two adjacent erosive efficacies

Strip width for a pocket with a texture

Width of erosive efficacy source

Footprint width

Width coordinate

Dimensionless width coordinate

Normalized locations of base arcs with respect to feature center for RMA

Longitudinal coordinate

Dimensionless longitudinal coordinate

Depth coordinate

Desired feature topography

Minimum depth of desired feature machined as nozzle moves during total time $T$

Derivative of $z$ with respect to $t$

Derivative of $z$ with respect to $x$

Constant depth for a channel machined by a constant scan speed of the source

Desired centerline depth

Measured shallow straight channel profile

Dimensionless depth coordinate 
Expected resulting shape of dimensionless profile

$z_{p t}^{*}$

Profile resulting from point source solution 


\section{Chapter 1 Introduction}

\subsection{Background and Motivation}

Abrasive jet micro machining (AJM) uses a jet of high speed abrasive particles to mechanically etch micro-features into components used in, e.g. in micro-fluidic, microelectromechanical systems (MEMS) and optoelectronics applications [1]. Figure 1-1 shows a typical AJM setup. A micro-Abrasive blaster mixes 10-50 $\mu \mathrm{m}$ granular particles (usually aluminum oxide) with air at $200-500 \mathrm{kPa}$ and passes the resulting mixture through a $0.4-1.5 \mathrm{~mm}$ diameter nozzle. A room dehumidifier, a refrigeration air dryer, and a desiccant filter on the inlet air are used to minimize the moisture in the powder reservoir. A variable speed rotary electric mixer is used in the reservoir to avoid powder clogging and particle agglomeration. The resulting etching rate depends on parameters such as air pressure, particle size, and nozzle shape, size and inclination. By manipulating the size, shape and velocity of the erosive 'footprint' of the jet on the surface, the final shape of the machined features can be controlled.

Traditionally, control of the shape of machined features has been limited to simply defining their bounding dimensions using patterned protective masks attached the target material. However, the ability to control feature shape within the micro-feature bounds would open up a wide variety of opportunities in the design of novel three dimensional micro-components. For example, in micro-fluidics, the channel cross-sectional shape can be a significant factor in the flow and heat transfer characteristics, and also affects probability of settling of micro-fluidic particles [2]. Moreover, although surface evolution models to predict feature shape given the input AJM process parameters already exist [3], the inverse problem of determining the AJM process parameters to achieve a desired shape has not yet been addressed. The lack of machining techniques for controlling feature shape and models for solving this inverse problem form the prime motivation for the work in this dissertation. 


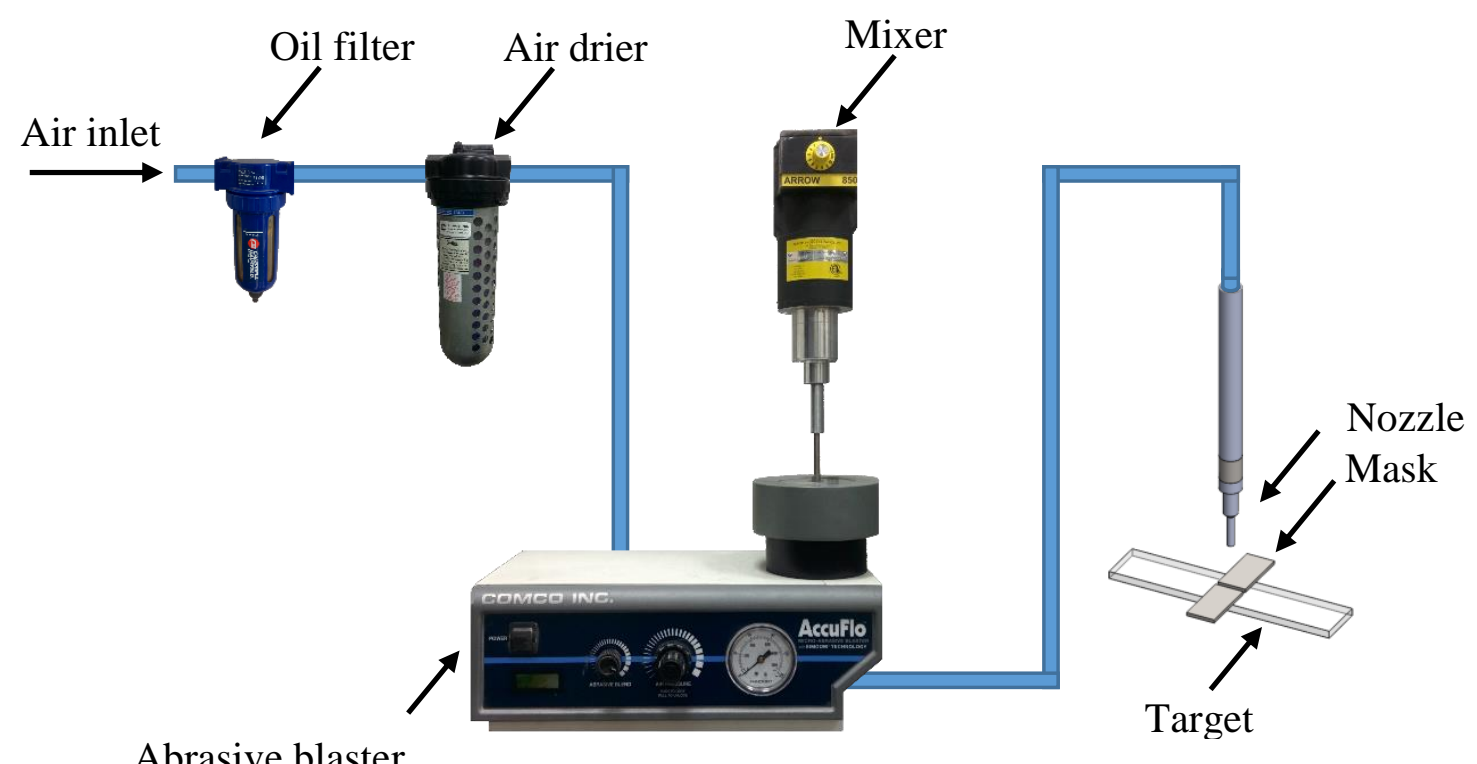

Abrasive blaster

Figure 1-1 Schematic of a typical abrasive jet apparatus.

\subsection{Literature Review}

In this section, a brief literature review is presented to facilitate a concise overview of the prevailing schools of thought on erosion mechanisms, and landmark work on AJM. It is intended to illustrate the areas where further research is required. More extensive literature reviews on specific topics are provided at the beginning of Chapters 2-6.

\subsubsection{Solid particle erosion mechanisms}

AJM removes material through solid particle erosion mechanisms, i.e. due to mechanisms that are associated with the impact of small particles at high speed. Solid particle erosion can be classified into brittle and ductile categories [4,5]. For a brittle material, material removal occurs due to deformation wear and fracture mechanism, with a maximum in erosion rate at perpendicular (normal) incidence. For ductile materials, the maximum erosion occurs at an oblique impact angle, where material removal due to cutting and ploughing mechanisms are maximized. As will be seen in Sections 4.3.3.3 and 4.3.3.4, this difference in dependence of erosion rate on angle of attack results in AJM micro-channels being V-shaped in brittle materials, and U-shaped in ductile materials. Therefore, a very brief review of typically observed erosion mechanisms is appropriate. 
There are different theories regarding the mechanics of material removal by solid particles. Among those theories, erosion by cutting, erosion by deformation wear, erosion by target melting, erosion by delamination, and erosion by crack propagation have received the most attention [6].

\subsubsection{Erosion by cutting}

Finnie [7] considered a particle's strike on a ductile surface as non-cutting if its flat surface makes contact, and cutting if its sharp edges contact the surface. In his pioneering model, the sharp edges of the rigid abrasive are assumed to cut and remove material in an ideal manner from a ductile material. Using a similar but modified approach, Hutchings [8] showed that at oblique impact angles, cutting or ploughing may occur when sharp particles impact mild steel. The erosion mechanism was found to be dependent on the impact angle of particles with respect to the target surface. High-speed photography was used to observe the erosion process. Although the presented theory was both quantitatively and qualitatively successful, there were some shortcomings. First of all, not all eroding particles are angular and second not all impacts give rise to detached chips of material. Papini and Spelt [9,10] created a rigid-plastic model of the impact of idealized rhomboid particles. The models were developed with the assumption of fully plastic target behavior and were able to predict the resulting crater shape for a variety of particle angularities. Takaffoli and Papini [11] successfully simulated chip formation and impact crater formation in erosion process using finite element and smoothed particle hydrodynamics (SPH) numerical models of more realistic multiple angularity particles. The results were verified by comparison with the kinematics and crater geometry resulting from impacts of rhomboid particles on a copper substrate. In another work, Takaffoli and Papini [12] modeled the impact of single particles on Al6061-T6 substrates using SPH. A gas gun was used to shoot angular particles in specific orientation. It was found that for normal angles of incidence, target material was mainly extruded and stacked at the edge of the crater made by the particle impact. Takaffoli and Papini $[13,14]$ presented a method of generating realistic particle geometries. This was used to model multiple non-overlapping and overlapping impact of particles on an A16061-T6 substrate. The crater size distribution was successfully modeled for both perpendicular and oblique angles of incidence. 


\subsubsection{Erosion by deformation wear}

Bitter [4,5] considered two mechanisms during solid particle erosion of both brittle and ductile materials. The first was due to repeated deformation of the target as a result of the particle collisions. The accumulated damage due to the repeated deformation eventually breaks off a piece of material. The second was due to the cutting action of the free-moving particles. It was found that in practice these two wear mechanisms were inseparable. For example, Hutchings et al. [15] launched steel spheres at oblique angles on a mild steel substrate to investigate the correlation between crater size, impact angle and sphere impact velocity. A model for crater formation was presented that could predict both the energy lost during the impact of spherical particles and the displaced volume of material. It was concluded that not all of the displaced material was removed from the target material. Jardret et al. [16] investigated the relation between the elastic behavior of material, contact mechanics and indentation process. The normal and tangential scratch forces were measured and the contact area during the indentation process was calculated by determining the penetration depth and the three-dimensional topography of the scratched surface. This made it possible to consider both elastic deformation and pile-up of material during solid particle erosion processes.

Rickerby and MacMillan [17] studied impact of WC-6\% Co spheres at normal incidence to an aluminum substrate. It was observed that material removal mechanisms were different at normal and shallow angles of incident. Oka et al. [18] proposed a solid particle erosion model based on the relationship between dynamic indentation and the surface hardness of the target. It was used as a foundation for other research on the attack angle dependence of erosion. For example, Oka et al. [19,20] reported that for ductile materials, cutting wear mostly occurs at low angles while at high angles deformation wear is the dominant erosion mechanism.

\subsubsection{Erosion by target melting}

The kinetic energy of a particle impinging a ductile material mostly converts to plastic deformation which dissipates in the form of heat. If this process occurs quickly and in a small area, the temperature can reach the melting point. This rise in temperature reduces the cohesive strength which results in easier material removal from the target [21]. Using electron microscopy, it is possible to authenticate that the melting has occurred during the machining [6]. For example, 
Andrews and Field [22] investigated mass loss of annealed copper target by shooting hardened steel spheres against the ductile target at temperatures between $70 \mathrm{~K}$ and $1350 \mathrm{~K}$. It was found that, although the target material started to lose its mass at temperatures over $900 \mathrm{~K}$, evidence of material melting in the form of small debris nevertheless was observed at all temperatures. It was concluded that the melting was a result of kinetic energy transferred from the sphere to the target during the impact.

\subsubsection{Erosion by delamination wear}

Suh [23] proposed theory of erosion by delamination for wear of metals. Based on this theory, dislocations at the surface of the material can cause subsurface cracks and voids. Shear deformation of the surface joins those cracks, eventually causing material erosion. It was concluded that adhesive, fretting and fatigue wear are all results of similar phenomena. It was also reported that the wear rate and the shear deformation of the surface layer are directly related.

Jahanmir [24] presented an approximate elastic-plastic solution to the stress problem around a subsurface microvoid during solid particle impact. The target was considered to be composed of a matrix containing harder inclusions. It was concluded that void forms in specific subsurface regions and depth at which void nucleated was directly related to impact velocity.

\subsubsection{Erosion by crack propagation}

Finnie [25] considered erosion of brittle materials as a result of crack propagation during solid particle impact. In case that the impact energy was large enough, a ring crack was developed around the impact area. As the loading was increased, the crack started to form a cone shape. This cone propagation eventually resulted in removal of the material. Wensink and Elwenspoek [26] proposed that the area beneath a sharp particle impact on a brittle surface consists of a plastically deformed region from which a radial crack forms. Their theory holds that during unloading, tensile stresses caused by the plastically deformed area will form lateral cracks that lead to material removal (Figure 1-2). 


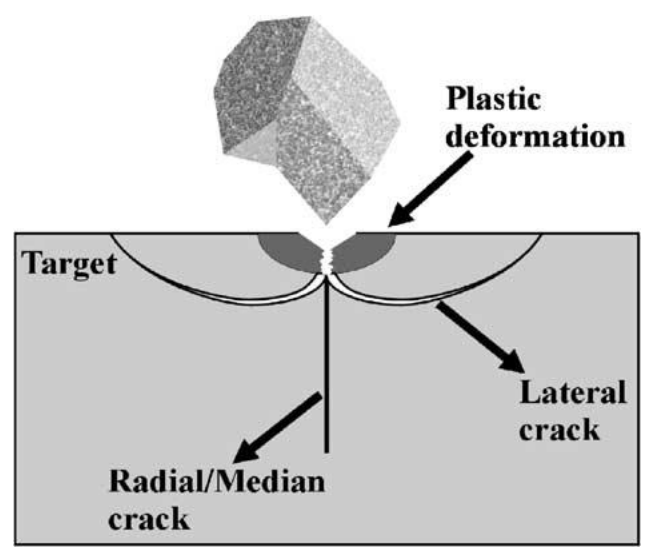

Figure 1-2 Schematic of crack formation during solid particle impact [26].

Slikkerveer et al. [27] presented a relation between different parameters of brittle indentation models. It was found that kinetic energy transfers perpendicular to the surface are responsible for lateral cracks, and thus erosion in the form of chipping.

\subsubsection{Mask materials used in abrasive jet micro-machining}

AJM involves not only the erosion of the target material, but also the patterned erosion resistant mask that is usually attached to the target to define the edges of the features to be machined. A variety of mask materials have been used in AJM. Pawlowski et al. [28] combined two polymeric mask techniques for use on glass substrates. The brittle epoxy resin SU8 and the elastic and thermo-curable polydimethylsiloxane (PDMS) were used sequentially. The former made for easier patterning because it utilized photosensitivity in its cure, while the latter provided a high erosion resistance, but at the cost of a multi-step procedure in which molds had to be produced from which the masks were cast. Performing AJM with $10 \mu \mathrm{m}$ diameter aluminum oxide particles, feature sizes of $20 \mu \mathrm{m}$ to $30 \mu \mathrm{m}$ were achieved. They also observed a reduction in the etching rate with a decrease in the openings (a 'blast lag') in the masks as they became smaller than ten times the particle size.

Wensink et al. [29] tested two polymers which could be lithographically structured and also examined metal masks. Ordyle BF400, an elastic negative resist foil was applied to a heated target and was developed using a $0.2 \% \mathrm{Na}_{2} \mathrm{CO}_{3}$ spray. The second polymer examined was polyamide, a negative liquid resist with a much higher erosion resistance than Ordyle BF40. Liao and Chen [30] used a photo etching method for creating a patterned mask on the target. After 
preheating the substrate, it was coated by polyvinyl alcohol and polyvinyl acetate (a water soluble resin) followed by another preheating and coating with a photosensitive polymer. Finally, pattern formation on the second layer was performed using AJM. After blasting, the second layer was peeled off after dissolving the first layer. This method had two advantages, a high resistance to powder blasting, and the ease of mask removal by peeling. However, a problem with underetching was observed which led to larger holes being machined than intended.

Metallic masks have also found use in AJM because of their high erosion resistance, allowing for higher resolution and higher aspect ratio features to be made than using e.g., polymeric masks. For example, Wensink et al. [29] used electroplated copper masks with AJM in order to create channels as small as $50 \mu \mathrm{m}$ wide on glass, and observed them to be highly erosion resistant. Ghobeity et al. [31] and Burzynski et al. [32] used steel shims to machine and model the surface evolution of AJM features in glass and PMMA with aspect ratios (depth to width) greater than one.

A number of authors have adapted self-adhesive elastomeric masks originally developed for sand-carving and sandblast etching applications to AJM applications. For example, Ghobeity et al. [33] used such masks to machine micro fluidic chips, compared their performance to chips made using conventional wet etching. They reported that since they could be easily patterned and applied, and since they resulted in a good machined feature resolution, the elastomeric masks had a high machining flexibility for a wide variety of applications, making them suitable for rapid prototyping applications. Despite these advantages, they were found to not be suitable for making high aspect ratio features, since they are relatively thin, erode quickly, and can deform elastically [34]. Burzynski et al. [32] modeled the effect of their wear on the surface evolution of features made using AJM, reported that the mask wear resulted in undesirable widening of the machined feature profiles in both brittle targets and ductile targets. In Chapter 6, elastomeric masks are used in a novel rotating mask configuration that extends their lifetime, whilst also increasing their flexibility to create a wider variety of features. A further review of mask utilization in AJM is presented in Section 5.1. 


\subsubsection{Modeling of feature shape resulting from AJM}

\subsubsection{Surface evolution model}

Thije Boonkkamp and Jansen [35] and Slikkerveer et al. [36] were the first to present a model for predicting the surface evolution of features machined using masked AJM in brittle materials. By taking into account the dependence of the instantaneous local erosion rate on the local angle of incidence of particles striking the sloped sidewalls of eroding features (Figure 1-3), they were able to derive the following surface evolution equation,

$$
z_{, t}=\frac{C}{\rho_{s}} V(x)^{k} \varphi(x)\left(1+z_{, x}^{2}\right)^{-k / 2}
$$

where $z_{, t}$ and $z_{, x}$ are the partial derivatives of the depth, $z, \rho_{s}$ is the density of the target material $\left(\mathrm{kg} / \mathrm{m}^{3}\right), \varphi(x)$ is the particle mass flux $\left(\mathrm{kg} / \mathrm{m}^{2} \mathrm{~s}\right)$ and $V(x)$ is the particle velocity distribution across the channel. $C$ and $k$ are erosion rate constants that are a function of particle size and shape, and can be found experimentally. For brittle materials, Equation (1.1) assumes that the erosion rate (mass of material removed per mass of erodent incident) depends on the velocity exponent, $k$, and the component of particle velocity and flux parallel to the local normal, i.e. erosion is proportional to energy transfers perpendicular to the surface. This dependence is reflected in the last term of Eq. (1.1).

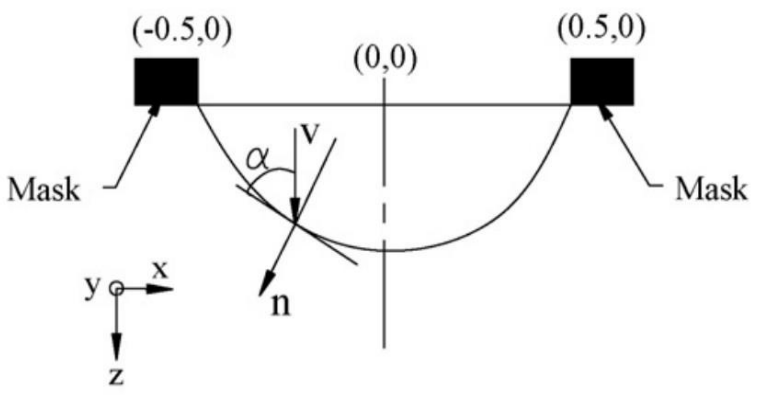

Figure 1-3 Schematic of coordinates used for masked channel cross-sectional profiles [37].

Equation (1.1) can be written in non-dimension form as [38] 


$$
z_{, t^{*}}^{*}=V^{*}\left(x^{*}\right)^{k} \varphi^{*}\left(x^{*}\right)\left(1+z_{, x^{*}}^{* 2}\right)^{-k / 2}
$$

where $z^{*}=z / L, x^{*}=x / L$ and $t^{*}=t / T$ are the non-dimensional depth, channel width and time, respectively. The time required to propagate the surface at $x=0$ to a characteristic depth, $L$, was defined as the time constant $T=\left(L \rho_{s} / C V(0)^{k} \varphi(0)\right)$. The characteristic length, $L$, can be conveniently chosen as the nozzle to surface standoff distance, $h$, for unmasked features, or the mask opening width, $w$, for masked features. The non-dimensional velocity and flux $V^{*}\left(x^{*}\right)$ and $\varphi^{*}\left(x^{*}\right)$ were found by normalizing using their values at $x=0$ (channel centerline).

For ductile materials, the impact angle dependency of erosion is different than brittle materials since the component of velocity parallel to the surface also causes erosion due to cutting mechanisms. Ghobeity et al. [39] showed that in this case, the surface evolution model becomes

$$
z_{, t^{*}}^{*}=\left(\left[V^{*}\left(x^{*}\right)\right]^{k} \varphi^{*}\left(x^{*}\right) \sqrt{\left(1+z_{, x^{*}}^{*}\right)}\right) g(\alpha)
$$

where $g(\alpha)$ is an empirical function that describes the impact angle dependence of the erosion rate. In principal, any curve fit of experimental data can be used as $g(\alpha)$, but the form suggested by Oka et al. [18-20] has been found to work well for a wide variety of metals, polymers and ceramics, i.e.

$$
g(\alpha)=(\sin \alpha)^{n 1}\left[1+H_{v}(1-\sin \alpha)\right]^{n 2}
$$

The constants $n_{1}$ and $n_{2}$ can be found by curve fitting of Equation (1.4) to erosion rate data. $H_{v}$ is the initial target hardness in GPa. The angle between the tangent to the channel surface in the $x-z$ plane and the $z$ component of the incident velocity vector, $\alpha$ (Figure 1-3), is given by [37] 


$$
\alpha=\left[\frac{\pi}{2}-\arccos \left(\frac{1}{\sqrt{1+z_{, x^{*}}^{* 2}}}\right)\right]
$$

Consequently, the erosion rate as a function of the local impact angle can be expressed as:

$$
E(\alpha)=g(\alpha) E 90
$$

where $E 90$ is erosion rate at normal incidence [19]. $g(\alpha)$ can be approximately determined experimentally by measuring the erosion rate on a surface due to a jet of particles incident at various angle of attacks. A nozzle with the smallest possible divergence should be used, and only shallow erosion scars should be made, in order to minimize the spread in local particle angles of attack.

These surface evolution equations thus allow the shape of the machined surface to be determined as a function of the jet erosive efficacy (Section 1.2.4) incident to the surface. Chapter 4 will present methods to solve the inverse problem, i.e. predicting the erosive efficacy to create a desired shallow machined shape.

\subsubsection{Numerical and computer models}

The time-dependent surface evolution models described by Equations (1.2) and (1.3) are in the form of partial differential equations which are generally difficult to solve using traditional analytical techniques. As a result, numerical methods have been used to solve them. Ghobeity et al. [39] and Getu et al. [37] solved these equations using a method of lines (MOL) approach implemented in Mathcad software. Others have used more sophisticated numerical methods. For example, Yagyu and Tabata [40] used a three-dimensional simulation for AJM of a glass substrate with a patterned mask. A cellular automaton algorithm was adopted which consisted of two orthogonal planes of two-dimensional cells. The simulation considered normal incidence of particles, a constant flux, no particle ricochet, no interference effects and no particle interactions with the mask edge. Shafiei [41] used a similar cellular approach, and discretized the surface to form a three dimensional grid of cubic cells. The simulation could simulate particle-particle and 
particle-surface collision and their kinematics. Ciampini et al. [42] presented a cellular automaton simulation for AJM of masked brittle materials. Particle to surface and mask collisions were modeled by tracking individual particles. The model made it possible to consider effects of spatial hindering, second strike and particle size in the predicted profiles. Burzynski et al. [1] presented a level set method approach to numerically solve the surface evolution equations associated with the AJM of unmasked and masked channels and holes in glass and PMMA. The level set method converts the equation of motion for a moving front (i.e. the target surface in this case) into solution of an initial value partial derivative equation. The level set method predicted surface evolution of the etched AJM profiles with better accuracy compared to previous MOL based models, and with lower solution times than cellular based methods.

\subsubsection{Erosive efficacy}

Erosive efficacy is a function of particle velocity and flux which defines the potential of an abrasive jet to do damage to a surface [39]. For a shallow profile, $z_{, x^{*}}^{*}$ in Eq. (1.2) approaches 0, and therefore a non-dimensional erosive efficacy, $E^{*}$, equivalent to the resulting non-dimensional shallow profile itself, can defined as

$$
E^{*}=z^{*}=\varphi^{*}\left(x^{*}\right) V^{*}\left(x^{*}\right)^{k}
$$

The erosive efficacy thus represents the shallow erosive 'footprint' left on the surface by a source of particles (a nozzle, a jet passing through a mask, etc.). In the presence of a mask, even if the velocity distribution is constant, the particle flux and consequently the erosive efficacy will not be constant because, depending on their particular size, only a certain proportion of particles that are close to the mask edge will reach the target surface. In other words, smaller particles will strike closer to the mask edge than larger ones, thus affecting the distribution of mass flux near the edge. Figure 1-4 shows spherical particles close to the mask edge. It can be concluded that for the particles to be able to successfully pass the mask opening they need to be at least one radius away from mask edge. Thije Boonkkamp et al. [35] presented this decrease in particle flux as a firstorder approximation (i.e. a linear decrease). 


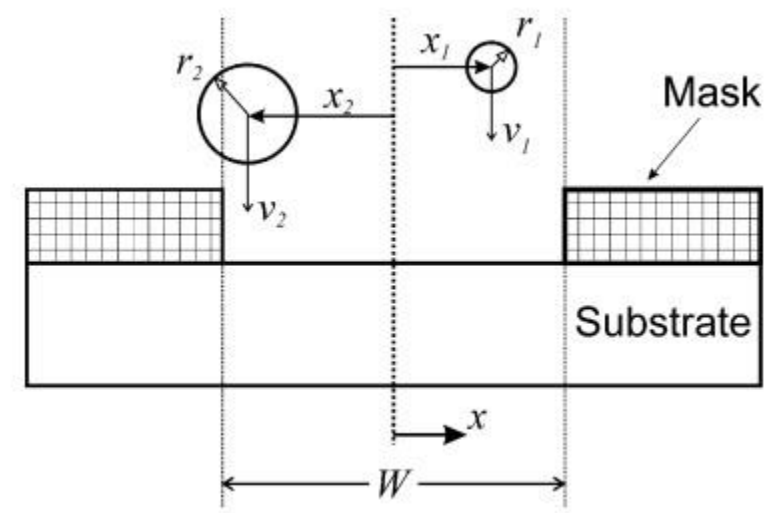

Figure 1-4 Two spherical particles approaching the mask opening [43].

$$
\varphi(x)=\left\{\begin{array}{lll}
x / \delta & \text { if } & 0 \leq x<\delta \\
1 & \text { if } \delta \leq x \leq 1-\delta \\
(1-x) / \delta & \text { if } 1-\delta<x \leq 1
\end{array}\right.
$$

In addition, a constant particle size and velocity distribution was assumed. However, the particle mass flux, velocity, and size distributions are not constant nor do they change linearly. When incorporated in the surface evolution equations his interpretation of erosive efficacy gave only fair accuracy for predicting feature shape up to an aspect ratio (depth to width) of $<0.5$. Beyond this aspect ratio, the fit was poor and the predicted channel and hole profiles showed sharp cusps along their center line. Ghobeity et al. [39] and Getu et al. [37] mitigated the cusp problem somewhat by adding a curvature-dependent smoothing term into the surface evolution equation. This term adjusts the predicted evolving speed of surface for highly curved regions of the profile, both for masked and unmasked holes and channels. To provide a more accurate estimate of the erosive efficacy distribution through a mask, Ghobeity et al. [43] presented an analytical model based on the measured particle size distribution and the mask opening width.

Ghobeity et al. [39] also introduced a semi-empirical method to define the net erosive efficacy through a mask opening. Following Eq. (1.7), a shallow eroded first-pass profile was fitted with an exponential function and used as the erosive efficacy in surface evolution equation. The advantage of this method was that the mask edge interaction effect, and particle size, velocity and flux distributions were all captured in the measured erosive efficacy. The predictions using this 
approach exhibited much better agreement to the experimental profiles compared to previous methods.

\subsection{Summary}

Abrasive jet micro machining is an effective technique for machining micro-features in a wide variety of materials. Channels machined by AJM in literature are limited to V-shaped and Ushaped cross sections for brittle and ductile materials, respectively. However, there is a demand in the next generation of micro devices to make more complex 3D shapes with multiple depth and controllable shape. Surface evolution equations are well developed to predict the shape of microchannels knowing the process parameters such as scan speed, nozzle trajectory, etc. However, there is no technique in literature to do the reverse, i.e. predict the required process parameters for a given desired shape. The existing ways in which erosive efficacy, and thus feature shape can be controlled have been limited to using masks or changing nozzle size. There is a need for methodologies to control erosive efficacy so that wider variety of shapes can be made. The objectives of this thesis are motivated by these shortcomings.

In Chapter 2, a shadow mask that is attached to the nozzle will be introduced that allows control of the erosive efficacy footprint width so that AJM can be used as a direct write technology. In Chapter 3 models to predict the profile of nontraditional W-shaped micro-channels machined by oscillation of the target will be presented. In Chapter 4 and Chapter 5 a new approach will be presented that can change the net shape of the footprint incident to the surface by controlling its traverse velocity, thus making direct writing of three-dimensional (multiple depths in same feature) possible. Chapter 6 will present a novel rotating disk apparatus that can produce almost any shape of erosive efficacy. The models that will be presented in Chapter 4 and Chapter 6 can predict the final shape of erosive efficacy of these two techniques, and also how to control process parameters to obtain a desired erosive efficacy. These predictions can be used in surface evolution equations of Section 1.2.3.1 to predict the final profiles of deeper micro-channel machined by AJM. 


\subsection{Objectives}

The main objective of this dissertation is to develop methodologies that allow the predictable sculpting of micro-features of desired shape. The following secondary objectives will serve as milestones to achieve this main objective:

$>$ Design, development and testing of a new AJM direct-write masking technique (Chapter 2).

$>$ An analytical model to predict the shape of micro-channels machined by oscillation of the target (Chapter3).

$>$ Development of an analytical solution for the inverse AJM problem so that the machining parameters required for direct-write of a wide variety of desired shapes can be predicted (Chapter 4).

$>$ Experimental verification of the inverse models by comparing typically desired machined channel and pocket shapes with those actually machined using the parameters predicted by the inverse model (Chapter 5).

$>$ Design, development and testing of a rotating mask apparatus that allows virtually any desired micro-channel shape (Chapter 6). 


\section{Chapter 2 Implementation of a shadow mask for direct writing in abrasive jet micro-machining}

This chapter is based on the following published paper:

*A. Nouhi, *M. R. Sookhak Lari, J. K. Spelt, and M. Papini. "Implementation of a shadow mask for direct writing in abrasive jet micro-machining." Journal of Materials Processing Technology, vol. 223, pp. 232-239, 2015.

* The first two authors contributed equally to this paper.

\subsection{Introduction}

Abrasive jet micro-machining (AJM) uses compressed air to create a jet of small particles to machine small-scale features in, for example, micro-fluidic, micro-electromechanical systems (MEMS) and opto-electronic components. AJM is attractive because of its low capital and operating cost, its very high etch rate on brittle materials such as glass, its ability to machine a wide range of materials, and its directional etch capability. Because the size of the jet footprint is typically on the order of several millimeters, a potential drawback of AJM is the need to apply erosion resistant masks in order to etch micro-scale features. Conventionally, the jet of particles is directed through an erosion resistant mask opening that is patterned to define the required features.

A number of devices have been recently fabricated using AJM in conjunction with patterned masks attached to the surface. For example, Belloy et al. [44] fabricated glass inertial sensors, Schlautmann et al. [45] electrophoresis capillaries, Park et al. [46] micro-molds in steel, and Yamahata et al. [47] ball valve micro pumps in glass. Masaki et al. [48] added a circular vane to slot nozzles in order to achieve a more uniform particle velocity distribution in order to machine large areas.

A number of different materials have been used to fabricate patterned masks. For example, Lomas et al. [49] developed a precision hot embossing metal mold in stainless steel by highresolution powder blasting in which PDMS and SU-8 were used as masks. Pawlowski et al. [28] used photolithographic methods to apply either epoxy or polydimethylsiloxane masks to glass 
substrates. This allowed feature sizes of $20-30 \mu \mathrm{m}$ to be achieved when blasting with $10 \mu \mathrm{m}$ diameter aluminum oxide particles. They also observed a reduction in the etching rate as the mask opening become smaller than ten times the particle size. Wensink et al. [29] also tested two polymers which could be lithographically structured on glass, and investigated the use of electroplated copper applied to glass over a titanium binding film. The resulting copper mask was highly erosion resistant, and channels of less than $50 \mu \mathrm{m}$ width were achievable. Liao and Chen [30] used various lithographic approaches to manufacture erosion resistant masks on glass and Ghobeity et al. [33] achieved feature sizes as small as 20-50 $\mu \mathrm{m}$, and channel aspect ratios of approximately 1 by using this method. Ghobeity et al. [39] also utilized a clamped mask consisting of two parallel $3 \mathrm{~mm}$ thick tempered steel strips in order to make straight channels in glass plates. The steel strips were milled to give sharp $90^{\circ}$ corners and smooth edges bounding the masked region. The use of these relatively thick steel strips improved channel edge definition by minimizing mask undercutting, providing greater resistance to lifting by the air stream and decreasing the number of particles striking the target surface at oblique angles after scattering from the corner edges of the mask.

As described above, conventional masks are either bonded or clamped to the target substrate. This limits flexibility and increases costs, since each device design requires a unique mask. This chapter introduces a new adjustable shadow mask system which is attached to a stationary nozzle, producing a narrow stream of abrasive particles to machine a target which is scanned in front of it, in order to produce the desired pattern. This avoids the cost and inconvenience of manufacturing, applying and removing fixed masks, as is the need for chemical solvents. The present work investigates the effects of shadow mask and nozzle stand-off, particle mass flux, and particle size on the resolution of the resulting features.

\subsection{Experiments}

\subsubsection{Shadow Mask Apparatus}

All experiments were conducted using an AccuFlo AF10 Micro-Abrasive Blaster, (Comco, Inc. Burbank, CA, USA) operating at $200 \mathrm{kPa}$ air pressure. A dehumidifier, a refrigeration air dryer, and a desiccant filter on the inlet air were used to minimize the moisture in the powder reservoir. A variable speed rotary electric mixer (Arrow Model 850, Arrow Engineering Co., Inc., 
Hillside, NJ, USA) was used in the reservoir to avoid powder clogging and particle agglomeration, and the powder level inside the reservoir was kept constant in order to reduce fluctuations in particle mass flux based on Ghobeity et al. [50].

Aluminum oxide $\left(\mathrm{Al}_{2} \mathrm{O}_{3}\right)$ powders with nominal diameters of $10 \mu \mathrm{m}$ (mean: $11.6 \mu \mathrm{m}$, standard deviation: $3.31 \mu \mathrm{m}$ ) and $25 \mu \mathrm{m}$ (mean: $24.1 \mu \mathrm{m}$, standard deviation: $16.2 \mu \mathrm{m}$; Comco, Inc. Burbank, CA, USA) were blasted on $3.3 \mathrm{~mm}$ thick borosilicate glass targets (Borofloat, Swift glass Co. Inc., Elmira, NY, USA). The glass samples were clamped to a programmable computercontrolled linear stage (Aerotech Inc., Pittsburgh, PA, USA) with a positioning resolution of 0.5 $\mu \mathrm{m}$. The stage permitted independent motion in two directions and could be used to provide curvilinear motion of the target under the fixed jet.

For the machining of straight channels, the clamp shown in Figure 2-1 was designed to hold the shadow mask and nozzle as the target was moved past them. A set of 3 equally-spaced springs between the nozzle and mask holder maintained a uniform contact pressure between the mask and the target surface. The nozzle was attached to the apparatus using a set screw, and the mask was clamped to the apparatus, in order to keep the jet centerline normal to the shadow mask and target. The nozzle-to-surface standoff was maintained at $20 \mathrm{~mm}$ unless otherwise mentioned (Figure 2-1). A relatively large $0.3 \times 3.8 \mathrm{~mm}$ rectangular nozzle (Comco, Inc. Burbank, CA, USA) was used with its long axis perpendicular to the slot of the mask opening (Figure 2-2) in order to minimize the effect of small misalignments of the jet center. 


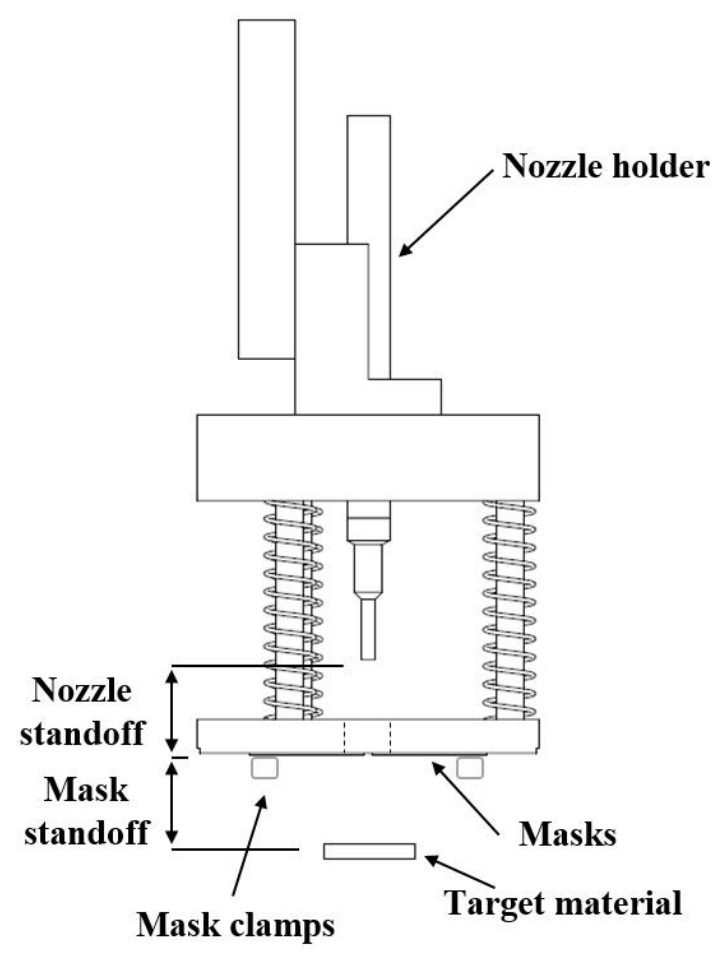

Figure 2-1 Shadow mask apparatus. Nozzle standoff $(N S)$ and mask standoff $(M S)$ were defined relative to the top of the mask. Only two of three springs shown.

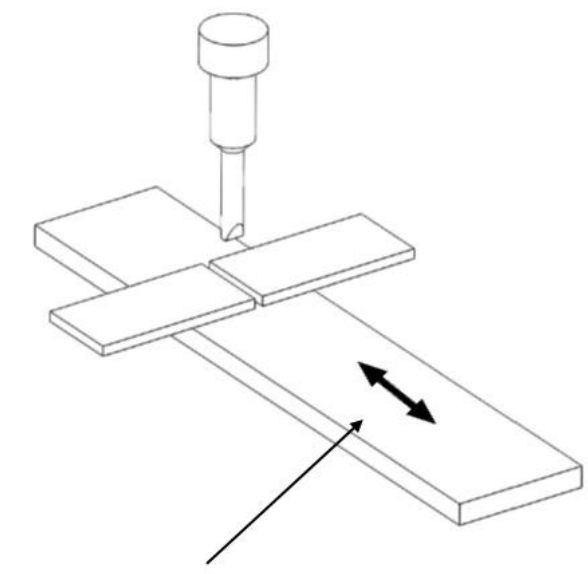

Direction of movement of target

Figure 2-2 Relative orientation of nozzle and shadow mask. 


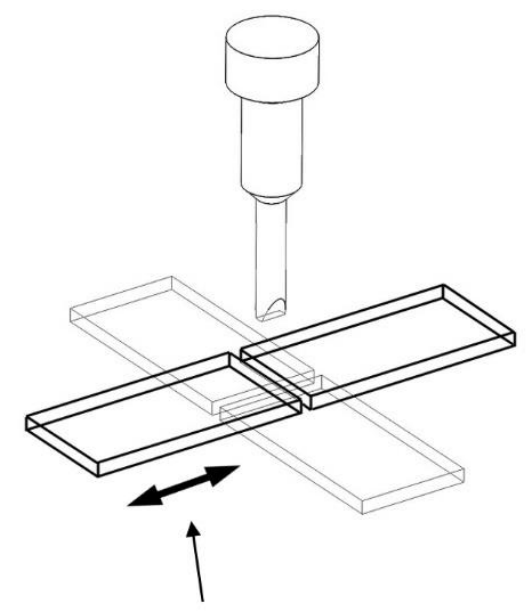

\section{Direction of movement of target}

Figure 2-3 Jet aperture for direct writing of curved paths made using two sets of crossed shadow masks.

The desired mask opening was obtained by placing a steel sheet of known thickness between the movable masks, and then clamping them in place. The mask opening was then photographed to determine its width with an accuracy of $\pm 6 \mu \mathrm{m}$ using digital image analysis. The mask openings for all experiments were in the range of 250 to $300 \mu \mathrm{m}$. The masks were investigated for any defect before and after each experiment and they were regularly changed to prevent any effect of mask erosion on the machined channels. Linear channels of $30 \mathrm{~mm}$ length were machined by moving the glass target under the mask at various mask standoff $(M S)$ and nozzle standoff $(N S)$ distances (Figure 2-1) in a straight line at a traverse velocity of $1 \mathrm{~mm} / \mathrm{s}$. All experiments under a given set of conditions were repeated on three specimens.

The mass flow rate before and after each experiment was measured by weighing the abrasive collected after 2 min of blasting into a cylinder covered with an air filter. The change in the mass flow rate during the course of a 2.5 min experiment was less than $10 \%$.

A final set of experiments was conducted using two sets of crossed masks to provide a square-shaped mask opening (Figure 2-3). This was used to machine linear and curvilinear channels. Table 2-1 summarizes the conditions used in the various experiments. 
Table 2-1 Summary of conditions used in each experiment.

\begin{tabular}{|c|c|c|c|c|c|c|c|}
\hline Experiment & $\begin{array}{c}\text { Particle } \\
\text { size }(\mu \mathrm{m})\end{array}$ & $\begin{array}{c}\text { Pressure } \\
(\mathrm{kPa})\end{array}$ & $\begin{array}{c}\text { Mask } \\
\text { opening } \\
(\mu \mathrm{m})\end{array}$ & $\begin{array}{c}\text { Powder mass } \\
\text { flow rate }(\mathrm{g} / \mathrm{min})\end{array}$ & $\begin{array}{c}\text { Nozzle } \\
\text { standoff } \\
(\mathrm{cm})\end{array}$ & $\begin{array}{c}\text { Mask } \\
\text { thickness } \\
(\mathrm{mm})\end{array}$ & $\begin{array}{c}\text { Mask } \\
\text { standoff } \\
(\mathrm{mm})\end{array}$ \\
\hline Clamped vs. shadow mask & 25 & 200 & 293 & 6.1 & 2 & 1.6 & 0 \\
\hline Effect of nozzle stand-off distance $(N S)$ & 25 & 200 & 270 & 5.9 & $1-2-3$ & 1.6 & 0 \\
\hline Effect of mask thickness & 25 & 200 & 270 & 6.2 & 2 & $0.8-1.6$ & 0 \\
\hline Effect of mask standoff $(M S)$ & 25 & 200 & 270 & 4.6 & 2 & 1.6 & $0-2$ \\
\hline Effect of particle size & $10-25$ & 200 & 290 & 4.7 & 2 & 1.6 & 0 \\
\hline Crossed-mask linear channels & 25 & 200 & $290 \times 290$ & 5.6 & 2 & 1.6 & 0 \\
\hline Crossed-mask curvilinear channels & 25 & 200 & $575 \times 575$ & 5.4 & 2 & 1.6 & 0 \\
\hline
\end{tabular}




\subsubsection{Measurement of channel cross-section and frosted region}

The channels were scanned using an optical profilometer (NANOVEA ST400 Micro Photonics Inc., Irvine, CA, USA) having a depth resolution of $25 \mathrm{~nm}$ and a lateral resolution of $0.1 \mu \mathrm{m}$, in order to determine the effect of various process parameters on the resulting channel dimensions and shape. It was found that the glass adjacent to the edges of the channels was slightly eroded ("frosted") as illustrated in Figure 2-4, likely due to particles penetrating under the masks. The width of this frosted region was measured using image analysis of photomicrographs as explained below. The erosion in the frosted regions was too small to produce a significant change in the average elevation of the glass target.

The frosted region complicated the definition of channel width. In the present work, the channel edge was defined as the point at which the average eroded depth of 10 consecutive profilometer measurement points, each $0.5 \mu \mathrm{m}$ apart, was equal to the depth of a crater created by a single particle of average size. According to Slikkerveer et al. [27] and by referring to Marshall et al. [51], the depth $a$ of a hemispherical crater is given by:

$$
a=\left(\frac{3 U}{2 \pi \bar{H}}\right)^{\frac{1}{3}}
$$

where $\bar{H}$ is the Vickers hardness of the target, and, $U$, the kinetic energy, is

$$
U=\frac{1}{2} \rho\left(\frac{4}{3} \pi R^{3}\right) v^{2}
$$

and $\rho, R$ and $v$ are the density, radius and velocity of an average erodent particle, respectively. Substituting Eq. (2.2) into Eq. (2.1), the depth of the indented zone becomes

$$
a=R\left(\frac{\rho v^{2}}{\bar{H}}\right)^{\frac{1}{3}}
$$


For borosilicate glass, the Vickers hardness is $5.68 \mathrm{GPa}$ and the density of aluminum oxide is $3,950 \mathrm{~kg} / \mathrm{m}^{3}$. Assuming a particle diameter $d_{p}=25 \mu \mathrm{m}$, the particle velocity model of Li et al. [52] was used to estimate an average particle velocity of $150 \mathrm{~m} / \mathrm{s}$ at a nozzle to surface standoff distance of $20 \mathrm{~mm}$. These values result in predicted crater depth $a=3.15 \mu \mathrm{m}$, which is approximately equal to $1 / 8^{\text {th }}$ of the particle diameter. Thus, the edge of the channel was considered as the point where the eroded depth was equal to $d / 8$, and the channel width was the average of three cross-sectional profiles, each on three separate specimens ( 9 measurements).

As shown in Figure 2-4, this definition of channel width typically agreed to within $10 \%$ with that obtained using a second method (slope method) whereby the edge of a channel was defined as the point, beginning from the sidewall of a channel and moving outward toward the periphery, where the absolute slope of a straight line fitted to 5 consecutive points spaced $5 \mu \mathrm{m}$ apart along a given channel cross-section was less than $10 \%$. Since the crater size method was easier to implement and was less sensitive to the resolution of the scanned profiles, it was used throughout the chapter.

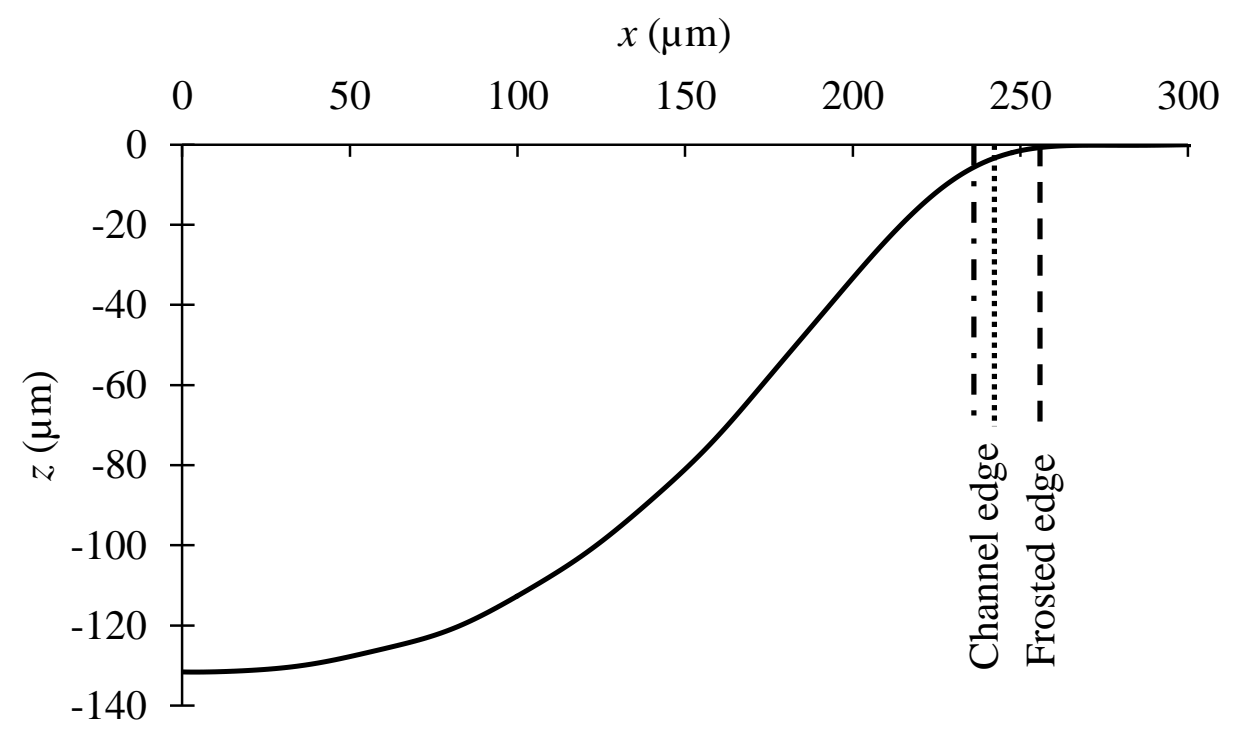

Figure 2-4 Location of edge of frosted area (dashed line) and a comparison of channel width based on (i) the slope method (dashed dotted line) and (ii) the channel crater size method (dotted line).

In order to obtain a measure of the width of the frosted region, three distinct subregions in a complete image of a channel (Figure 2-5) could be roughly recognized by differences in their 
color; i.e. an uneroded region relatively far from the channel (lightest), a frosted zone (grey), and finally the channel itself (dark grey). Once the channel width for a given channel was determined as described above, it was centered within the dark area, as shown in Figure 2-5 (the innermost two lines representing the channel width), and all pixels within the channel width were assigned totally black greyscale values. The outer edges of the frosted region were determined by performing image segmentation using Matlab (The Mathworks, Inc.), adjusting the threshold greyscale value until the greyscale of all pixels in the uneroded region were visually judged to be included. The pixels in this segmented area, which represented the uneroded area, were then also set to black. This resulted in the frosted regions being white, while the channel and surrounding uneroded glass were black, as shown in Figure 2-6. The average width of the white areas was then determined by multiplying the image width (vertical dimension of Figure 2-6) by the ratio of the white to black areas. Finally, the width of the frosted zone on each side of a channel was considered as half of the measured total width. The reported frosted widths were the average of the values on 3 separate specimens. Figure 2-4 shows the typical location of the frosted edge on a cross-sectional profile. While these definitions were somewhat subjective, they provided a consistent means to assess the dependence of the channel and frosted area widths on the process parameters. 


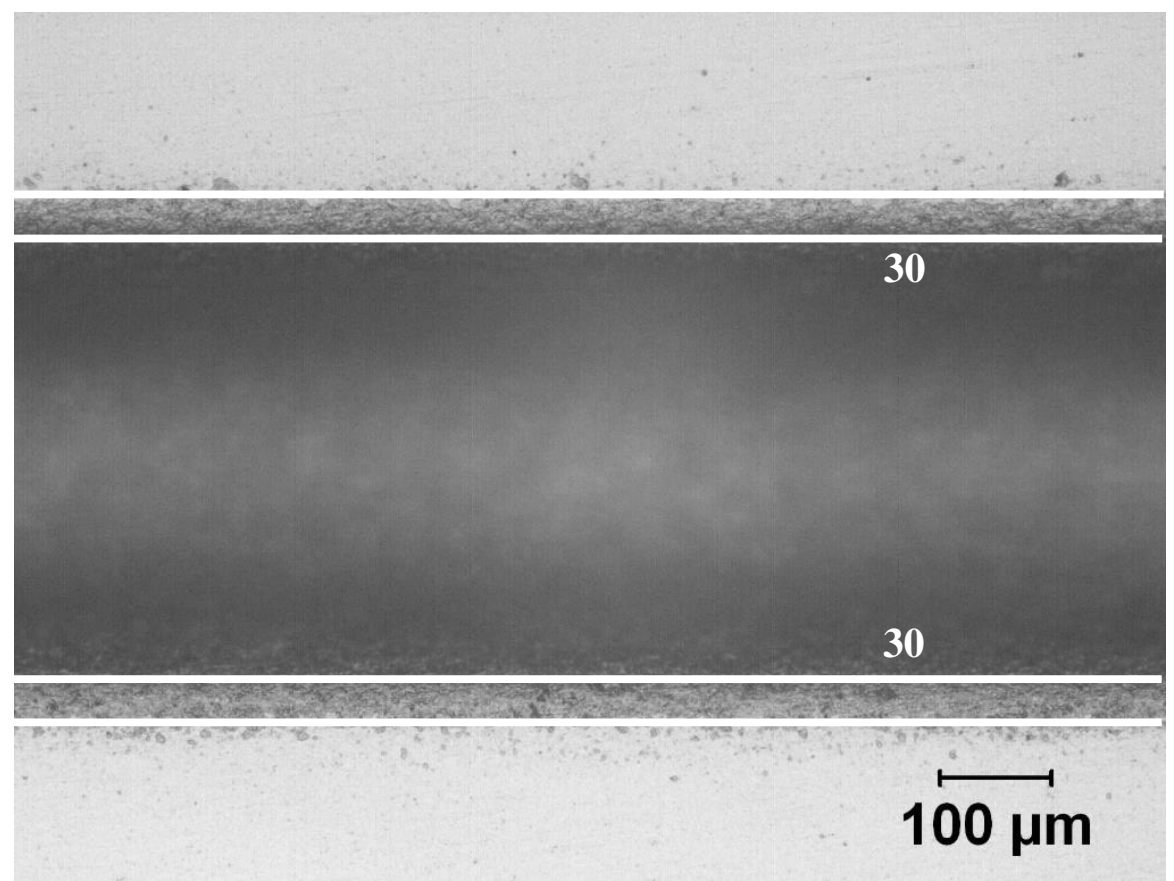

Figure 2-5 Top view of glass channel blasted at $200 \mathrm{kPa}, 20 \mathrm{~mm}$ nozzle standoff, with a contacting crossed shadow mask having a square opening $260 \mu \mathrm{m}$ on a side. The $30 \mu \mathrm{m}$ wide frosted regions are indicated.

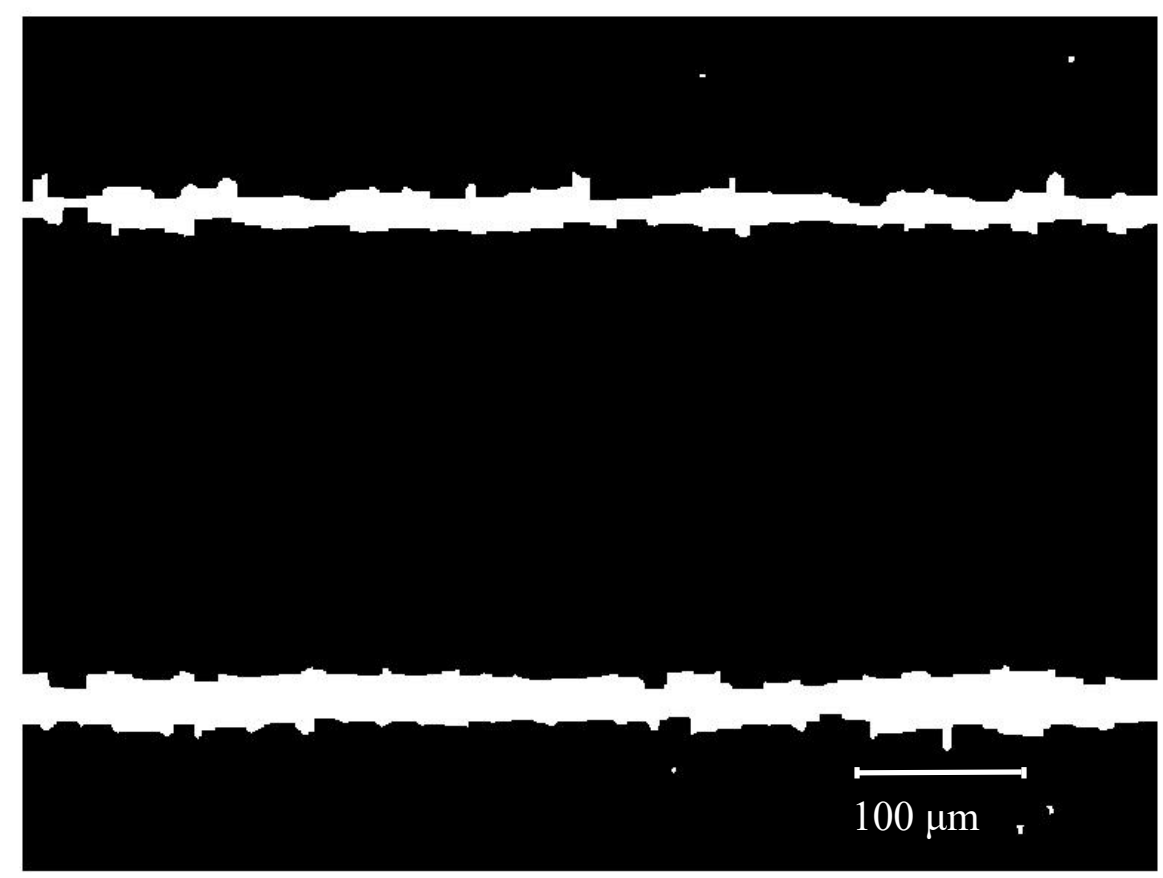

Figure 2-6 Segmented image of Figure 2-5 showing the frosted regions in white. 


\subsection{Results and discussion}

\subsubsection{Clamped vs. shadow mask}

Figure 2-7 compares the measured cross-sections of typical channels made using either the shadow mask of Figure 2-2 sliding on the glass surface (i.e. mask standoff=0 in Figure 2-1) or a traditional steel mask that was clamped to the glass. In both cases the mask opening was $293 \mu \mathrm{m}$, the mask thickness was $800 \mu \mathrm{m}$, and the nozzle standoff was NS=20 mm. It is evident that the shadow mask produced slightly wider and shallower channels than did the clamped mask. Depending on the number of passes, the channels machined using the shadow mask were approximately 3-15\% wider and 2-24\% shallower than those using made with the clamped masks. This widening occurred because particles could penetrate under the shadow mask and strike the surface between the mask and the target, something that is less likely to happen with clamped masks. Even though the shadow mask was pressed against the target, it had a standoff equal to approximately the diameter of the largest particles in the abrasive mixture, since these particles became trapped under the contacting shadow mask as the target surface moves. The smaller particles in the powder were then able to strike the surface under the shadow mask creating the frosted zone.

It is hypothesized that the channels were shallower when using the shadow mask for similar reasons; i.e. the leakage of particles and air under the masks resulted in fewer particles striking the surface within the mask opening. While there was no noticeable frosting of the glass at the periphery of the channels when the mask was clamped, the shadow mask produced frosted bands on either side of the channel that were approximately $60 \mu \mathrm{m}$ wide, because particles could penetrate under the mask. 


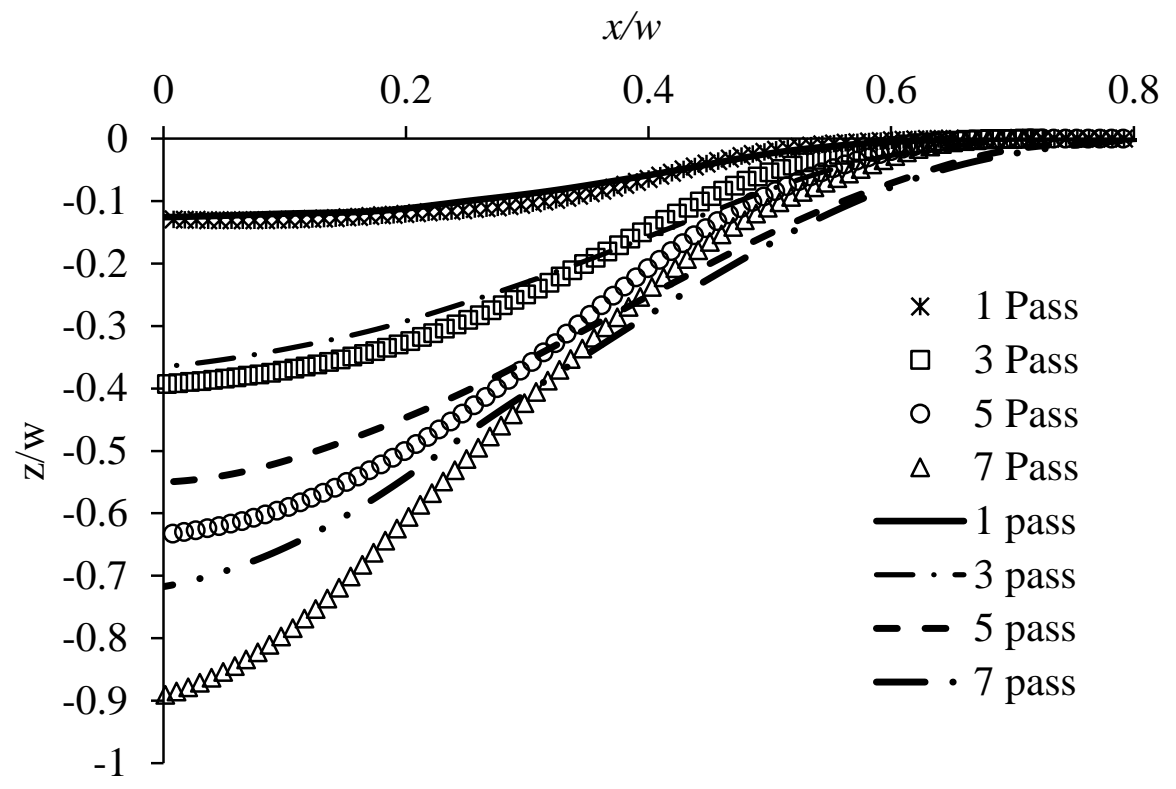

Figure 2-7 Cross-sectional profiles of channels machined using various number of passes and the shadow mask of Figure 2-2 in contact with the surface (solid and dashed lines) or a clamped mask (symbols). Dimensions normalized using the mask opening, $W=293 \mu \mathrm{m}$. Only half of each symmetric profile is shown. Mask thickness of $800 \mu \mathrm{m}, N S=20 \mathrm{~mm}$.

\subsubsection{Effect of nozzle standoff distance}

The effect of the nozzle standoff distance was investigated for the shadow mask of Figure 2-2 having an opening of $270 \mu \mathrm{m}$ with the mask and surface in contact (i.e. $M S=0$ ), and $N S=10,20$ and $30 \mathrm{~mm}$ (Figure 2-1; conditions of Table 2-1). Figure 2-8 and Figure 2-9 show that increasing $N S$ from $10 \mathrm{~mm}$ to $30 \mathrm{~mm}$ resulted in approximately $8 \%$ shallower and $7 \%$ narrower channels, and a $20 \%$ decrease in the width of the frosted region, Figure 2-10. The changes in channel depth, width and frosting were relatively small, but statistically significant ( $\mathrm{t}$-test, $\mathrm{p}<$ 0.05). These effects were attributable to the reduction in the particle flux through the mask opening that occurred with increasing nozzle standoff distance due to the divergence of the jet plume which can be found in work presented by Papini et al. [53]. This resulted in less material removal, both in the center and at the periphery of the channel, and produced fewer particles undercutting the mask edge to form the frosted regions. The slight asymmetry that was observed in the results was due to an imperfect nozzle alignment. 


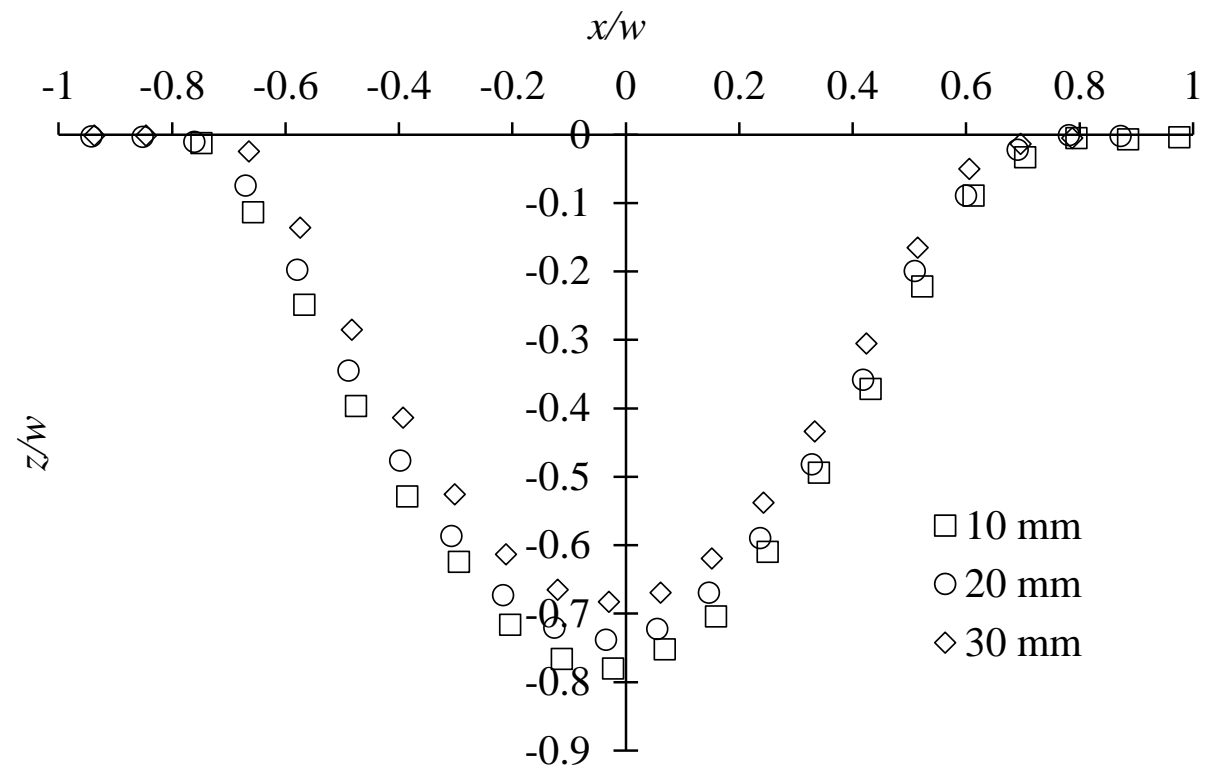

Figure 2-8 Comparison of typical channel profiles obtained after 4 passes of the shadow mask of Figure 2-2 at three nozzle standoff distances (Figure 2-1). Depth and width normalized by mask opening $W=270 \mu \mathrm{m}$.

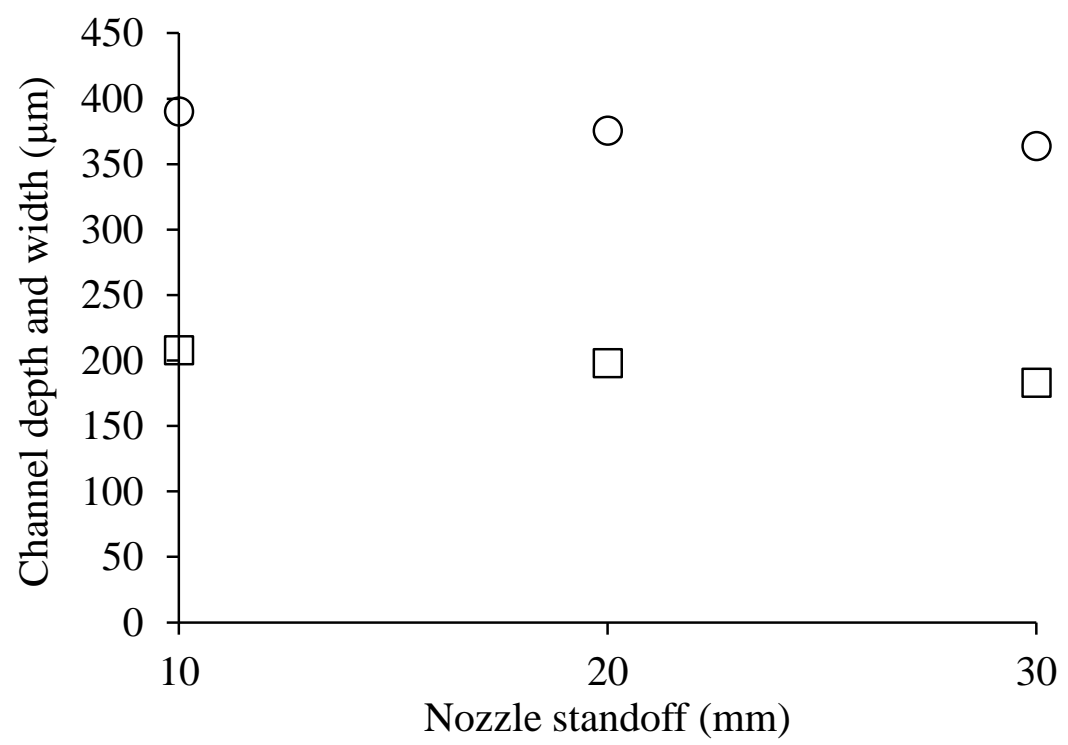

Figure 2-9 Average channel depth (squares) and width (circles) at three nozzle standoffs. Scatter bars representing \pm 1 standard deviation for 9 measurements ( 3 measurements on each of 3 specimens) were small enough to fit within the symbols. 


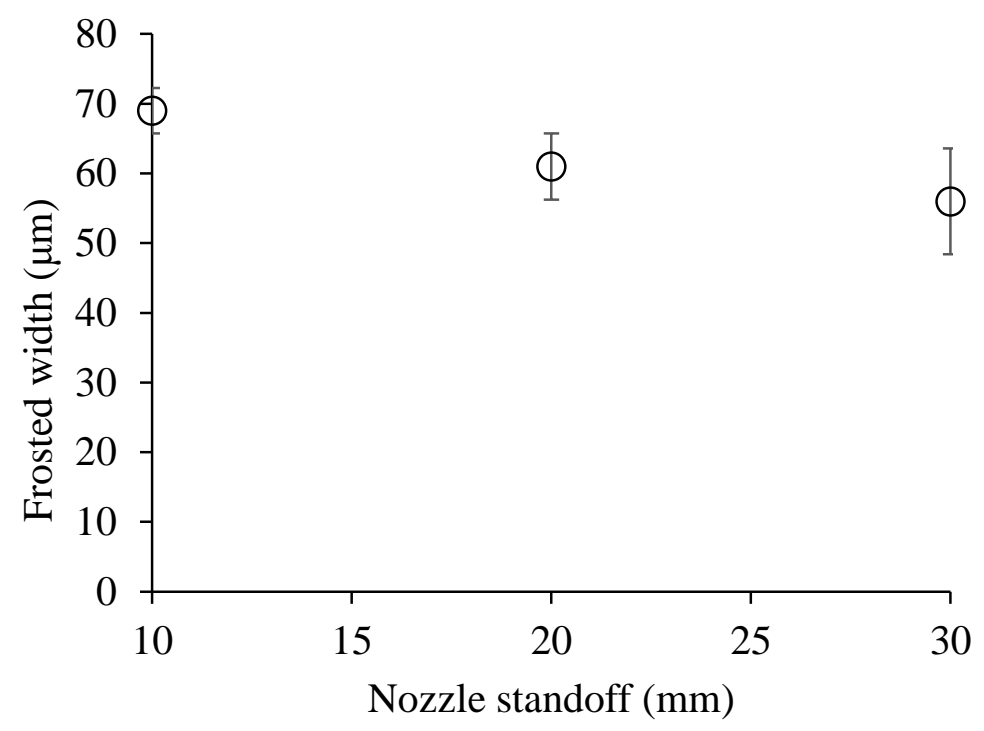

Figure 2-10 Average frosted width at three nozzle standoffs (Figure 2-1). The scatter bars represent \pm 1 standard deviation for 9 measurements ( 3 measurements on each of 3 specimens).

\subsubsection{Effect of shadow mask thickness}

The effect of shadow mask thickness on the channel profiles was investigated using 0.8 and $1.6 \mathrm{~mm}$ thick contacting shadow masks of the type shown in Figure 2-2, with an opening of $270 \mu \mathrm{m}$. Figure 2-11 and Figure 2-12 show that the thicker mask produced statistically significant decreases (t-test, $\mathrm{p}<0.05)$ in both the channel depth (17\% decrease) and width (4\% decrease). Figure 2-12 shows that the frosting width also decreased, by more than two fold when the mask thickness increased. These trends can be attributed to a reduction in the particle flux capable of erosion due to differences in particle ricochet from the mask edges, as shown in Figure 2-13. In the case of the thicker mask, it is more likely that particles which strike the edge of the mask will ricochet onto the opposite mask and lose most of their kinetic energy. Thinner masks permit more of the incident particles to reach the target surface with sufficient kinetic energy to produce erosion. Therefore, thicker shadow masks have the advantage of producing channels with much smaller frosted regions, albeit at the expense of a slightly reduced etch rate. 


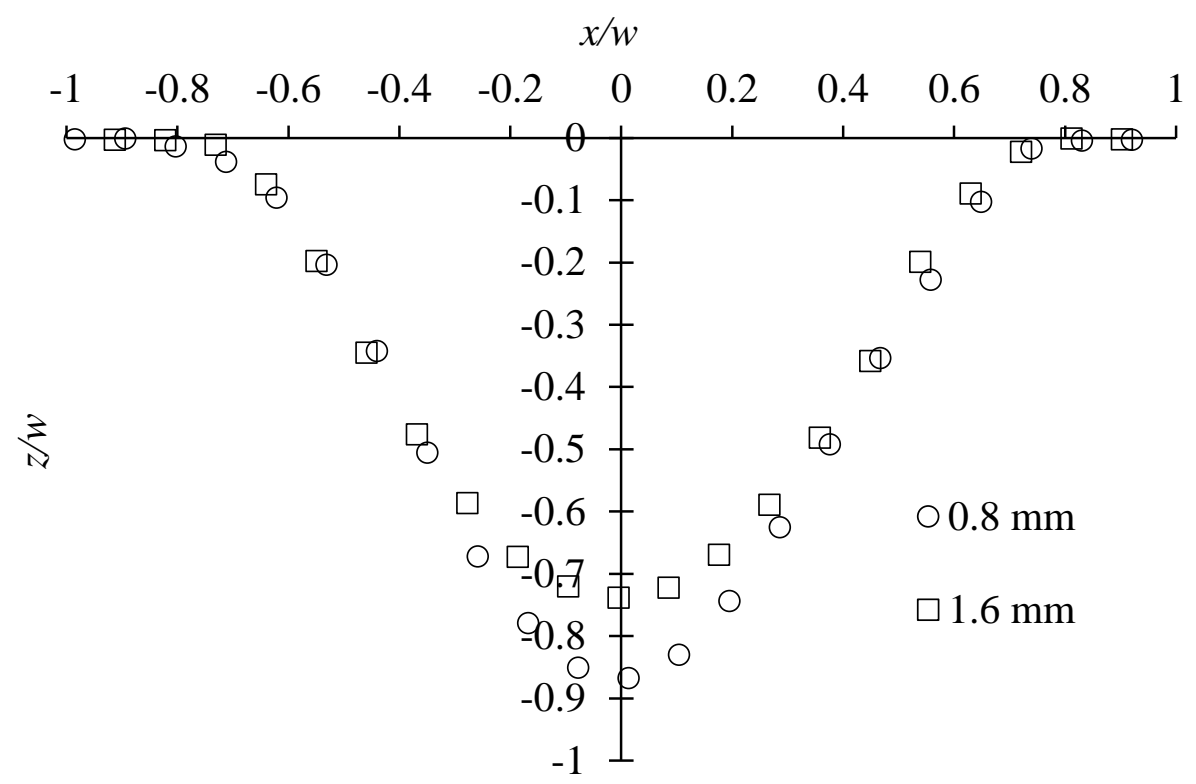

Figure 2-11 Effect of mask thickness on typical channel profiles obtained after 4 passes of the shadow mask of Figure 2-2. Channel depth and width normalized by mask opening $W=270 \mu \mathrm{m}$.

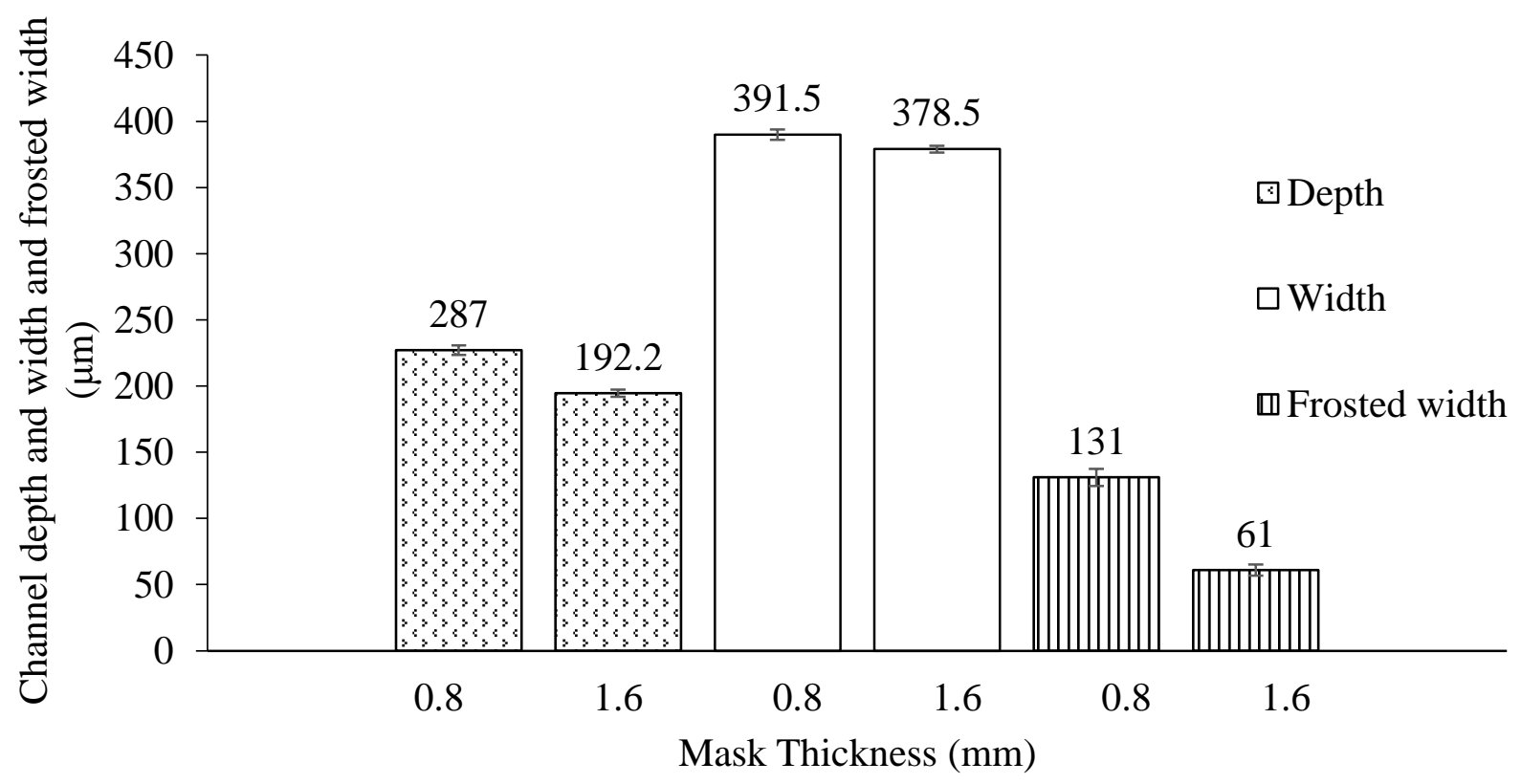

Figure 2-12 Average channel depth, width and frosted width for mask thicknesses of 0.8 and 1.6 $\mathrm{mm}$. The scatter bars represent \pm 1 standard deviation for 9 measurements ( 3 measurements on each of 3 specimens). 


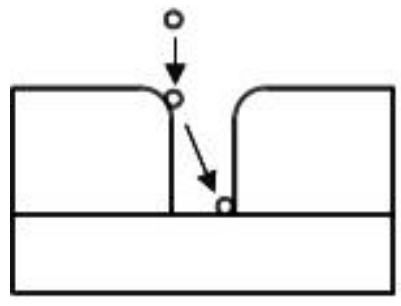

(a)

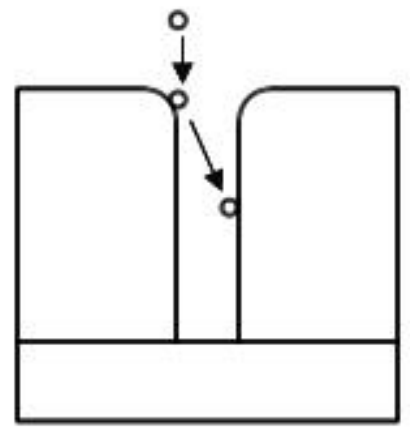

(b)

Figure 2-13 A particle striking the edge of a thin mask (a) is more likely to strike the surface, while that striking a thick mask (b) collides with the opposing mask.

\subsubsection{Mask standoff}

The effect of a $2 \mathrm{~mm}$ shadow mask standoff was investigated using the shadow mask of Figure 2-2 with a mask opening of 270 $\mu \mathrm{m}$. Figure 2-14 and Figure 2-15 show that introducing a $2 \mathrm{~mm}$ gap between the mask and the glass resulted in statistically significant changes (t-test, $\mathrm{p}<$ $0.001)$ in the depth (20\% decrease), width ( $7 \%$ increase), and frosted zone width (70\% increase) of the channels. The decrease in depth was mainly due to a reduction in the surface flux due to increased jet spreading and a decrease in the particle velocity due to drag. When the mask was not in contact with the surface, particles were decelerated quickly by the drag of the relatively quiescent air encountered after passing through the shadow mask opening. Using a modification of Li's model [52], it was found that this additional drag decreased the particle velocity from approximately $159 \mathrm{~m} / \mathrm{s}$ to $143 \mathrm{~m} / \mathrm{s}$. Assuming that the erosion, $E$, depends on the normal component of particle velocity, $V_{n}$, raised to an exponent of $K=1.43$ for glass with $\mathrm{Al}_{2} \mathrm{O}_{3}$ particles, where Ghobeity et al. [39] presented its equation as $E=C V_{n}^{1.43}$, where $C$ is a constant, and letting all other experimental conditions such as mass flux and particle size be the same, the predicted decrease in depth due to this velocity decrease was 14\%. This is in fair agreement with the actual decrease of $19 \%$, particularly since this estimate does not consider the additional effect of the decrease in surface flux over the $2 \mathrm{~mm}$ standoff.

The increase to a $2 \mathrm{~mm}$ mask standoff also allowed particles ricocheting from the mask edge to strike the target surface beneath the mask, thereby explaining the observed widening of the channel and the larger frosted zones. 


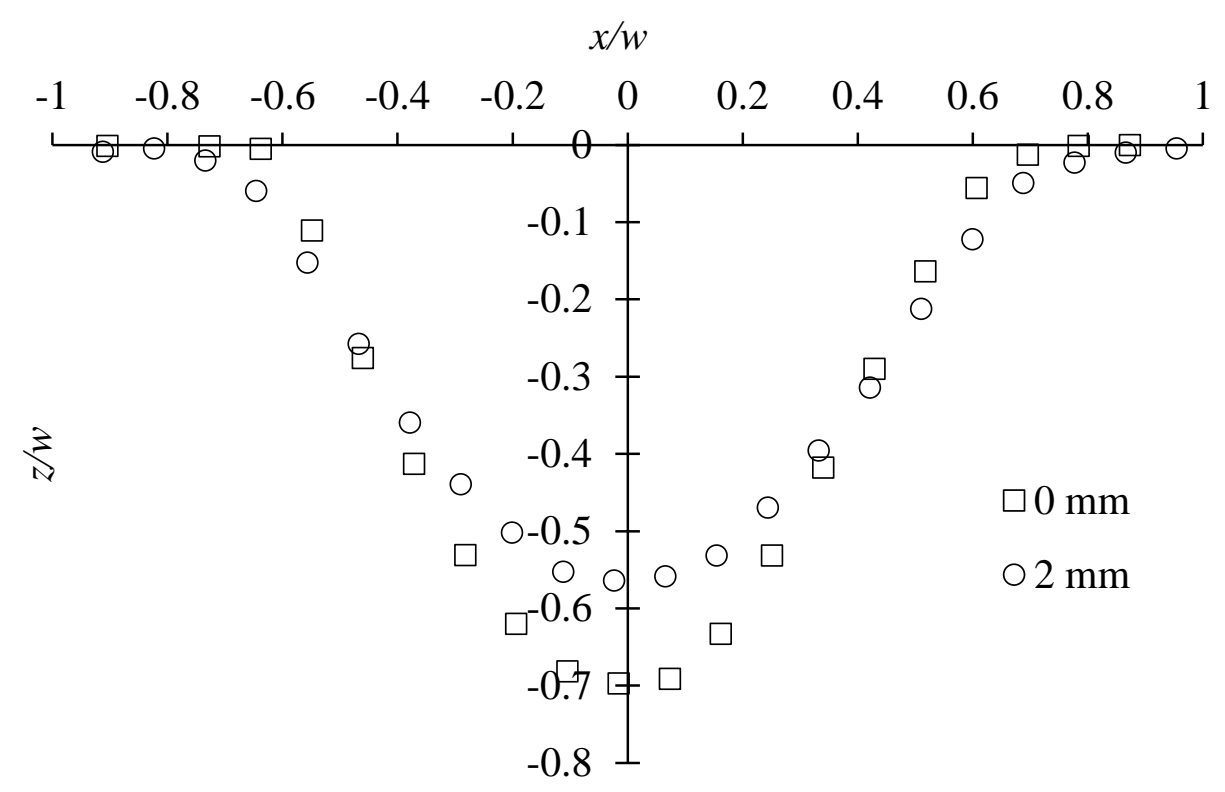

Figure 2-14 Typical channel profiles obtained after 4 passes of the shadow mask of Figure 2-2 using mask-to-surface standoff distances of 0 and $2 \mathrm{~mm}$ (Figure 2-1). Channel depth and width normalized by mask opening $W=270 \mu \mathrm{m}$.

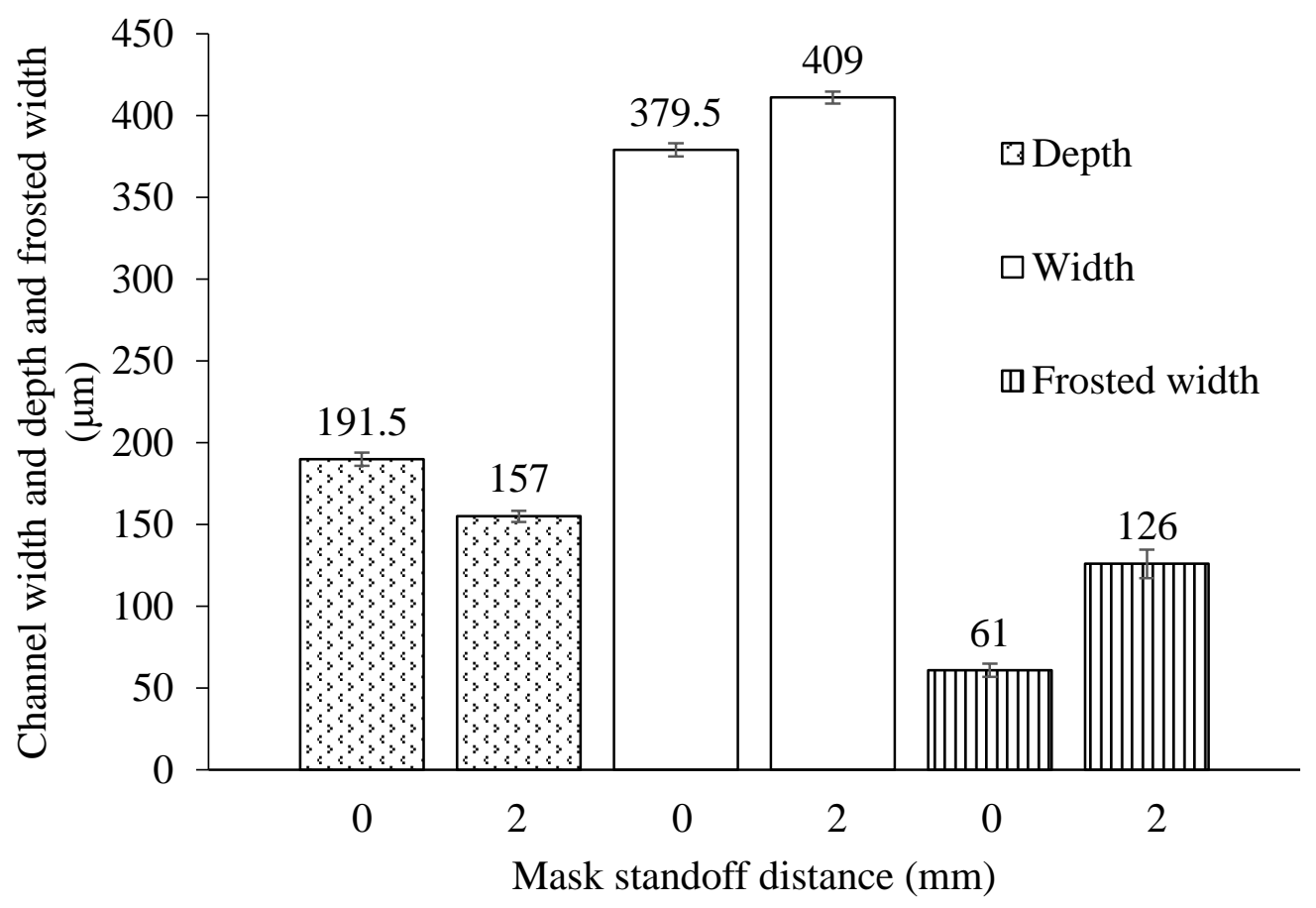

Figure 2-15 Average depth, width and frosted width for mask standoffs of 0 and $2 \mathrm{~mm}$. The scatter bars represent \pm 1 standard deviation for 9 measurements ( 3 measurements on each of 3 specimens). 


\subsubsection{Effect of particle size}

The effect of particle size was studied using 10 and $25 \mu \mathrm{m}$ alumina powder blasted at 200 $\mathrm{kPa}$ through a contacting shadow mask of the type shown in Figure 2-2 with an opening of 290 $\mu \mathrm{m}$. This produced very similar mass flow rates of 4.49 and $4.7 \mathrm{~g} / \mathrm{min}$ for the 10 and $25 \mu \mathrm{m}$ powder, respectively, and therefore the erodent doses delivered to the surface were nearly the same for both particle sizes. Increasing the particle size resulted in deeper and narrower channels, as shown in Figure 2-16 and Figure 2-17. Increasing the particle size from 10 to $25 \mu \mathrm{m}$ increased the depth by $25 \%$ and decreased the width by $8 \%$, both of which were statistically significant changes (t-test, $\mathrm{p}$ $<0.005)$. Recalling that the mass flow rates were nearly identical for both particle sizes, the increase in depth was mostly due to the greater velocity of the $25 \mu \mathrm{m}$ particles $(159 \mathrm{~m} / \mathrm{s}$, using the model presented by Li et al. [52]) compared to the $10 \mu \mathrm{m}$ particles $(141 \mathrm{~m} / \mathrm{s})$. Using the same analysis as in Section 2.3.4 (i.e, assuming that the erosion, $E$, depends on the normal component of particle velocity, $V_{n}$, raised to an exponent of $K=1.43$ for glass) with both sizes of particles, and considering the slight difference in mass flow rates noted above, the predicted increase in depth due mainly to this velocity increase was $24 \%$, which agrees well with the measured value of $25 \%$.

The increase in channel width with decreasing particle size is a well-known phenomenon and was presented by Ghobeity et al. [43], which is due to the fact that smaller particles can pass closer to the mask edge without striking the mask than can larger particles.

There was a near doubling of the frosted width when the particle size was increased from 10 to $25 \mu \mathrm{m}$ (Figure 2-17). As explained previously, since the shadow mask was sliding on the target, erodent particles became trapped under the mask and raised it slightly. This elevation was greater for $25 \mu \mathrm{m}$ particles than for $10 \mu \mathrm{m}$ particles, and consequently more of the smaller particles in the distribution were able to reach beneath the mask and produce frosting. 


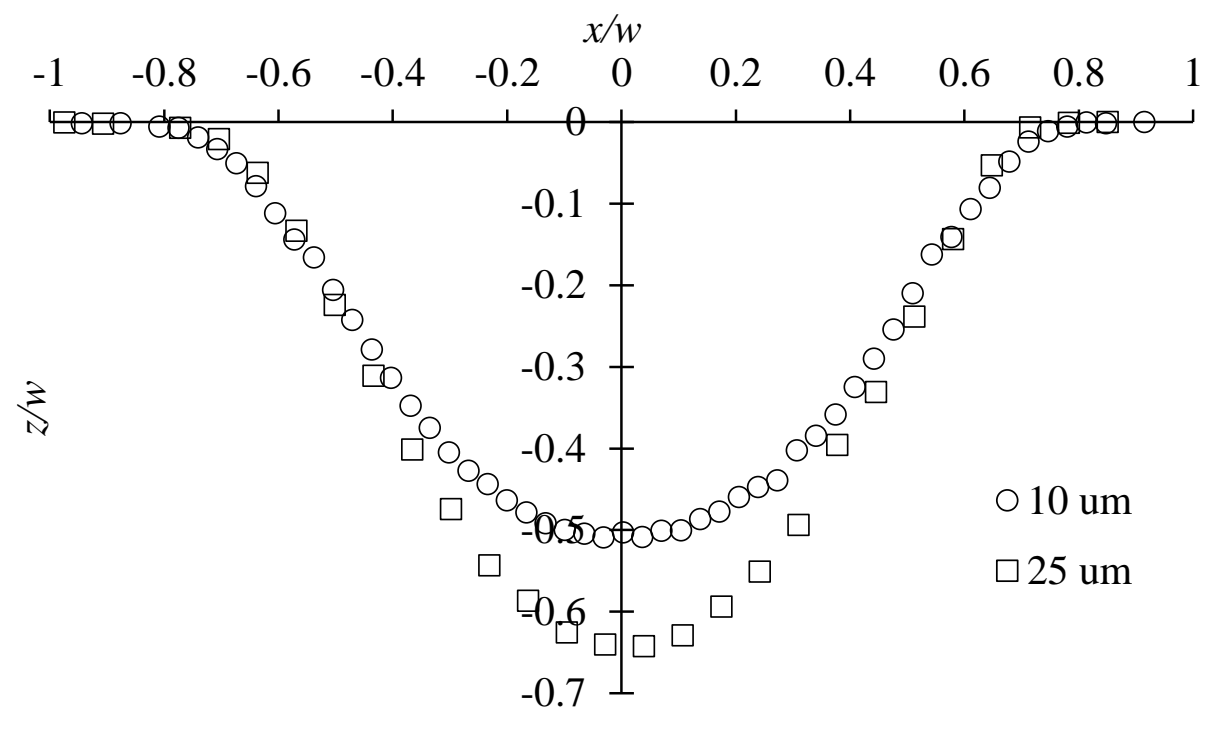

Figure 2-16 Channel profiles obtained after 4 passes of the shadow mask of Figure 2-2 using 10 and $25 \mu \mathrm{m}$ particles. Channel depth and width normalized by mask opening $W=290 \mu \mathrm{m}$.

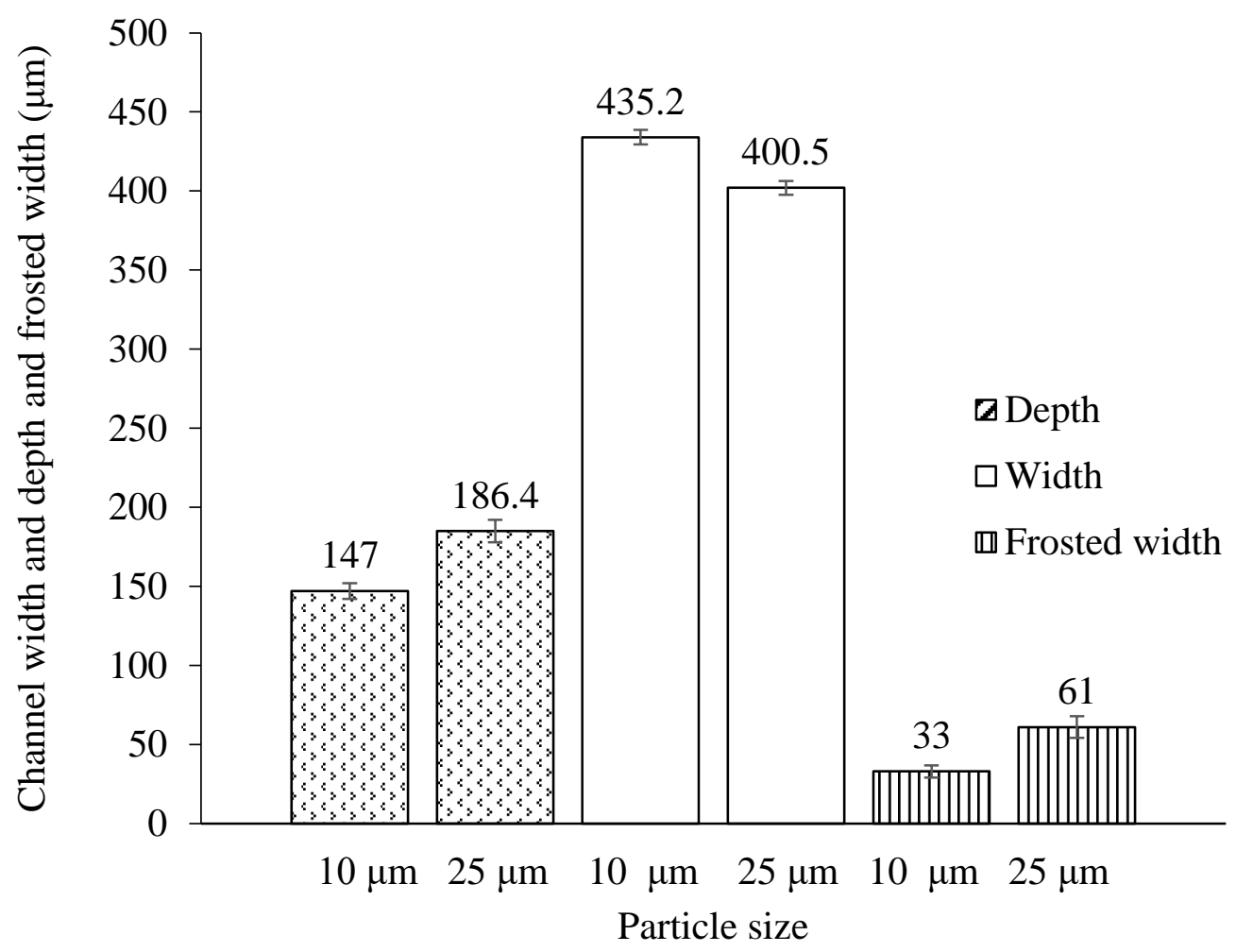

Figure 2-17 Average channel depth, width and frosted width for particle sizes of 10 and $25 \mu \mathrm{m}$. The scatter bars represent \pm 1 standard deviation for 9 measurements ( 3 measurements on each of 3 specimens). 


\subsubsection{Crossed shadow masks}

The shadow mask of Figure 2-2 produced a narrow impacting erodent jet, but with a fanshaped spread in its scanning direction due to the plume divergence in the narrow direction of the $0.3 \times 3.8 \mathrm{~mm}$ rectangular nozzle. This restricted the arrangement of Figure 2-2 to the machining of straight channels as shown. In order to achieve a more compact abrasive jet that was narrower in all directions - one with greater potential to be used as a direct write abrasive jet micromachining technology - a crossed arrangement of two mutually perpendicular sets of shadow masks was utilized (Figure 2-3).

Typical channel profiles made under identical conditions using crossed and uncrossed contacting shadow masks are shown in Figure 2-18. The average depth using the crossed mask was found to be $9 \%$ smaller than for the uncrossed mask (average of 3 measurements on each of 3 channels). This difference was found to be statistically significant ( $\mathrm{t}$-test, $\mathrm{p}<0.005)$. The channel widths for the two arrangements were, however, essentially the same; i.e. a $4 \%$ difference which was statistically insignificant (t-test, $\mathrm{p}>0.25$ ).

The decrease in the channel depth was likely due to the reduction in particle velocity with the crossed mask because of the increased effective mask standoff from the top set of masks to the surface. In addition, the crossed mask reduced the total particle dose delivered to the surface from the much smaller opening.

As shown in Figure 2-19, utilizing the crossed mask decreased the length of the jet footprint, thereby reducing the particle dose in the direction of scanning. This led to a reduced frosted zone size compared to the regular shadow mask since fewer particles were available to leak between the lower set of masks and the surface. The average width of the frosted zone using the crossed mask was the lowest of all experiments, approximately $30 \mu \mathrm{m}$, which $50 \%$ less than the frosted width for a regular shadow mask $(61 \mu \mathrm{m})$. The asymmetry in the crossed-mask footprint in Figure 2-19 occurred because of the air gap, equal to the thickness of the bottom masks, that was introduced between the top set of masks and the surface, which allowed some spreading of particles in the direction of the bottom slot (Figure 2-3). 


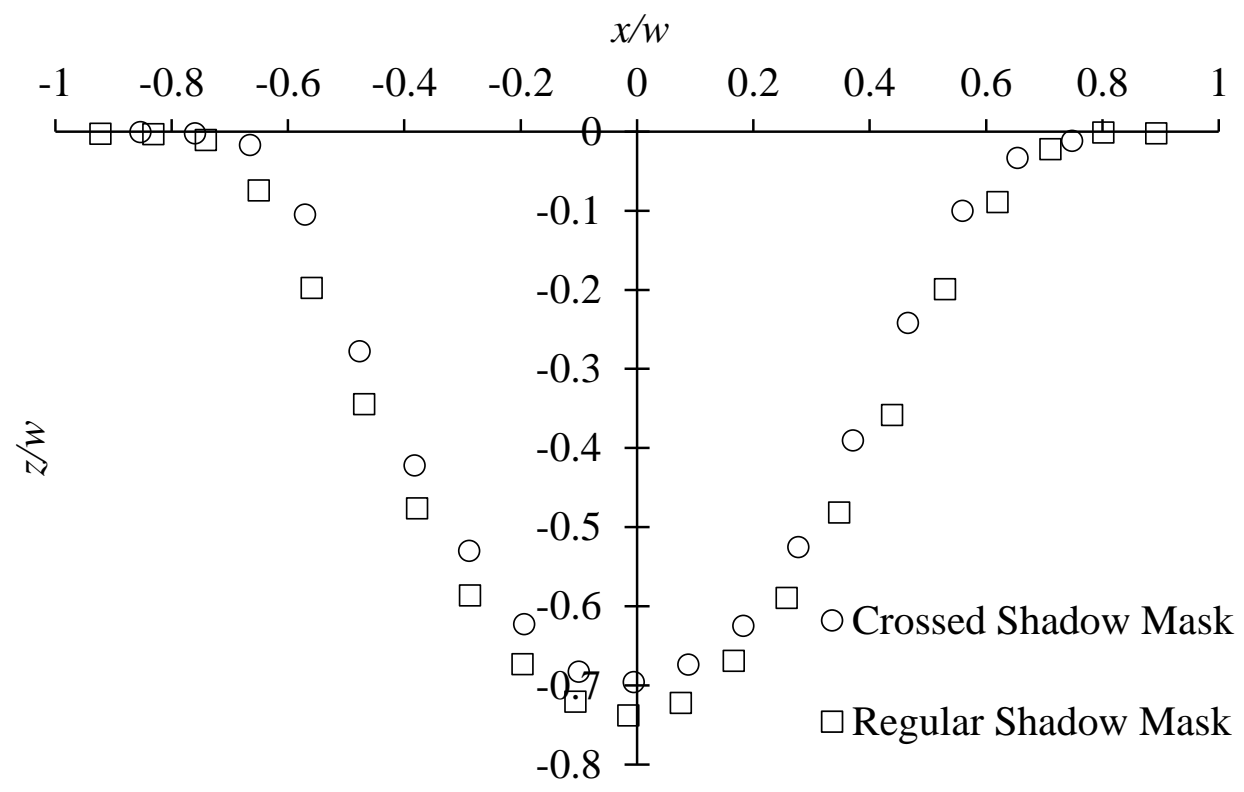

Figure 2-18 Profiles of channels made after 4 passes of the crossed shadow mask (Figure 2-3) and the regular shadow mask (Figure 2-2). Channel depth and width normalized by mask opening $W=290 \mu \mathrm{m}$.

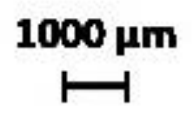

Figure 2-19 Footprints of the erodent jets produced using the crossed shadow mask (left) and the regular mask (right). For the crossed mask, the slot of the top set of masks was in the vertical direction.

Figure 2-20 shows an example of the direct writing ability of this technique for the machining of complex curved patterns. The width and depth of the lines in the machined logo are approximately, $700 \mu \mathrm{m}$ and $90 \mu \mathrm{m}$ respectively. 


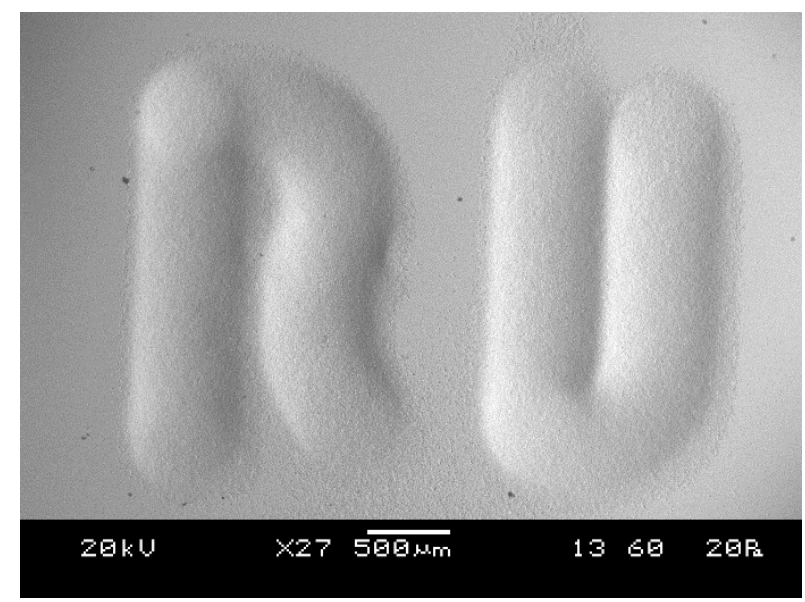

Figure 2-20 Ryerson University logo machined in glass using direct writing technique with crossed shadow masks.

\subsection{Summary}

A novel adjustable shadow mask has been developed consisting of two parallel metal strips attached to a nozzle for the abrasive jet mico-machining of straight channels. The shadow mask eliminates the need for costly and time consuming mask fabrication and attachment to the substrate, and allows for the straightforward adjustment of the mask opening. Compared to a traditional mask attached to the surface, the micro-channels machined using the shadow mask were found to be wider, and were bounded by a frosted regions adjacent to the channel edges. The effects of nozzle standoff, mask standoff, particle size and mask thickness on the channel width, depth and size of the frosted region were investigated and could be explained in terms of changes in the particle velocity and impact location. Increasing nozzle standoff and mask thickness, and decreasing mask standoff and particle size led to the narrowest features with the least amount of frosting. Taking this into account, it was possible to machine micro-channels that were only approximately $10 \%$ wider than those machined using traditional masks up to an aspect ratio of approximately 0.5 . Under these conditions, the frosted regions appeared in bands approximately $60 \mu \mathrm{m}$ wide. By using two sets of crossed masks, the channel width remained unchanged, but it was found that the frosted zone size decreased by 50\% (30 $\mu$ m wide bands). It was demonstrated that this crossed-mask configuration had the additional advantage that it could also be used to direct write curved features. 
The shadow mask that was developed in this chapter will be used in Chapters 3-5 to direct write micro-channels and other features with non-traditional topographies. 


\section{Chapter 3 Control of micro-channel cross-sectional shape in the abrasive jet micro-machining of ductile and brittle materials}

Portions of this chapter are based on the following published conference paper:

M. R. Sookhak Lari, and M. Papini. "Control of micro-channel cross-sectional shape in the abrasive jet micromachining of ductile and brittle materials." 11th International Conference on Micro Manufacturing, USA, 2016.

\subsection{Introduction}

There has recently been increasing interest in the fabrication of micro-fluidic devices having micro-channels with different cross-sectional areas from traditional isotropic wet etching. Flow characteristics and the probability of micro-fluidic particles settling are dependent on the channel cross-sectional shape [2]. Despite the increasing interest, there are very few techniques currently available to sculpt channel cross sections of desired geometry. Wilson et al. [54] showed that circular micro-fluidic channels in PDMS can be made by combining micro-milling and soft lithography. Kumi et al. [55] presented high speed multiphoton absorption polymerization (MAP) in PDMS for fabrication of different micro-fluidic channel cross-sections. Futai et al. [56] made bell shaped micro-channels in PDMS by a backside diffused-light exposure technique. Anisotropic chemical etching can be performed on silicon wafers, but the range of shapes that can be etched is limited by the orientation of the silicon crystal planes [57]. All of these techniques are limited since they are complex, time consuming, cannot create continuously curved features, and are useful for limited materials.

Abrasive jet micro-machining (AJM) can be used to machine three-dimensional (3D) features without having to deal with mentioned shortcomings of the other methods. The directional and gradient etch capability of AJM can be used to machine non-traditional shaped micro-channels [58]. AJM uses a jet of small particles to machine features for micro-fluidic, microelectromechanical systems (MEMS) and opto-electronic components. Proper control of the jet parameters such as the impact angle, the particle mass flux (mass per unit area per unit time), and 
the particle velocity, shape and size makes it possible to control the mechanical erosion of the substrate material [35]. Other advantages of AJM over other more traditional micro-machining techniques include low capital and operating cost, its very high etch rate on brittle materials such as glass, and its ability to machine a wide range of materials [59].

Typically, channels made using AJM on brittle materials such as glass with the jet at perpendicular incidence to the mask opening have a V-shaped profile [35]. However, channels machined in ductile materials, such as polymers, have a more U-shaped profile [37]. The differences in shape arise due to differences in the maximum erosion impact angle, i.e. for brittle erosive systems, at perpendicular incidence, and for ductile erosive systems, at an oblique impact angle [26]. Investigators have used AJM with the jet at perpendicular incidence to manufacture a variety of devices including glass inertial sensors [44], electrophoresis capillaries [45], microfluidic chips [33], micro-molds in steel [46], flat panel displays [60,61], high aspect ratio and complex monolithic structures in glass [58] and arrays of PZT pillars in order to manufacture piezocomposites [62]. Ghobeity et al. [33] presented a comparison between AJM and a chemical etching technique in the micro-fabrication of micro-fluidic chips and found the performance of AJM to be comparable to chemical etching in some respects.

There has been extensive work on predicting the shape of evolving micro-channels machined using AJM with a stationary nozzle at perpendicular incidence. The earliest were the mathematical models by Boonkkamp et al. [3] and Slikkerveer et al. [36]. These surface evolution models were subsequently refined by Ghobeity et al. [39] where they presented analytical and experimental methods to predict the erosive efficacy, a measure of the distribution of potential of the jet to erode a target surface, through the mask openings used in AJM. Burzynski et al. [1] used level set methods to model the surface evolution of unmasked and masked channels and holes machined in glass and polymethylmethacrylate using AJM. Ciampini and Papini [42] have also used cellular automaton methods to model more complex phenomena occurring in the AJM of glass. Finally, Ghobeity et al. [39] presented and modelled a technique to machine relatively large (> 1mm) multi-depth planar areas and transitional slopes in glass using AJM.

There are some examples of three-dimensional (3D) micro-features in the literature that were made by exploiting the directional etch capability of AJM. Examples include ball valve micro- pumps in glass [47], W-shaped micro-channels [63], and suspended structures such as 
accelerometers, millimeter size cantilever beams, complex micro-structures and monolithic microstructures $[44,58]$ in glass.

The directional etch capability of AJM makes it an ideal candidate for creating nontraditional micro-channel shapes. In this chapter, a target oscillation technique is introduced that allowed the maskless direct-writing of micro-channels having $\mathrm{W}, \mathrm{U}$ and trapezoidal shaped crosssections. Models to predict the shapes are also developed, and shown to predict measured microchannel cross sectional profiles in borosilicate glass and polymethylmethacryalate (PMMA) with good accuracy.

\subsection{Experiments}

\subsubsection{Apparatus}

An AccuFlo AF10 Micro-Abrasive Blaster, (Comco, Inc. Burbank, CA, USA) operating at $200 \mathrm{kPa}$ air pressure was used for all experiments. In order to minimize the fluctuation in particle mass flux, the techniques recommended in Ref. [50] were used. Two programmable computercontrolled linear stages (Aerotech Inc., Pittsburgh, PA, USA) with positioning resolutions in two independent perpendicular directions of $0.5 \mu \mathrm{m}$ were used to move the target relative to the stationary nozzle. More details on the experimental setup can be found in [64]. Borosilicate glass (Borofloat, Swift glass Co. Inc., Elmira, NY, USA), and polymethylmethacrylate (PMMA) (type ACRYLITE $^{\circledR}$ FF, CYRO Industries, Rockaway, NJ, USA) target materials were used to represent brittle and ductile, respectively, erosive systems. The abrasive powder was aluminum oxide $\left(\mathrm{Al}_{2} \mathrm{O}_{3}\right)$ (Comco, Inc. Burbank, CA, USA) of $25 \mu \mathrm{m}$ nominal diameter (normal distribution with mean and standard deviation of $24.1 \mu \mathrm{m}$ and $16.2 \mu \mathrm{m}$ respectively).

Two different direct-write AJM techniques were evaluated to avoid the need for attaching patterned masks to the surfaces. In the first, the developed adjustable shadow-mask system of Chapter 2 was used. The system utilizes adjustable masks which are attached to a rectangular nozzle $(0.3 \mathrm{~mm}$ by $3.8 \mathrm{~mm})$ and made to contact the surface, in order to create a jet footprint whose width can be controlled. Figure 3-1 shows a simplified schematic of the concept. The actual system, which utilizes springs to ensure continuous contact between mask and surface, is described

in more detail in Chapter 2. The mask opening was controlled in the range of $150-450 \mu \mathrm{m}$ by using feeler gauges, resulting in erosive footprints in the range of 250-550 $\mu \mathrm{m}$. The nozzle to surface 
standoff for the shadow mask machining experiments was kept constant at $20 \mathrm{~mm}$ while the mask to surface distance was $<100 \mu \mathrm{m}$.

In the second technique, a much smaller $460 \mu \mathrm{m}$ round nozzle was used with no mask, and with a very small (100 $\mu \mathrm{m}$, controlled using a feeler gauge) nozzle to surface standoff distance, in order to limit the footprint of the jet to $\sim 540 \mu \mathrm{m}$. The obvious advantage of this technique is the lack of the need for a mask which may erode and thus need replacing periodically. For both maskless machining and machining with the shadow mask, the nozzle was held normal to the target surface.

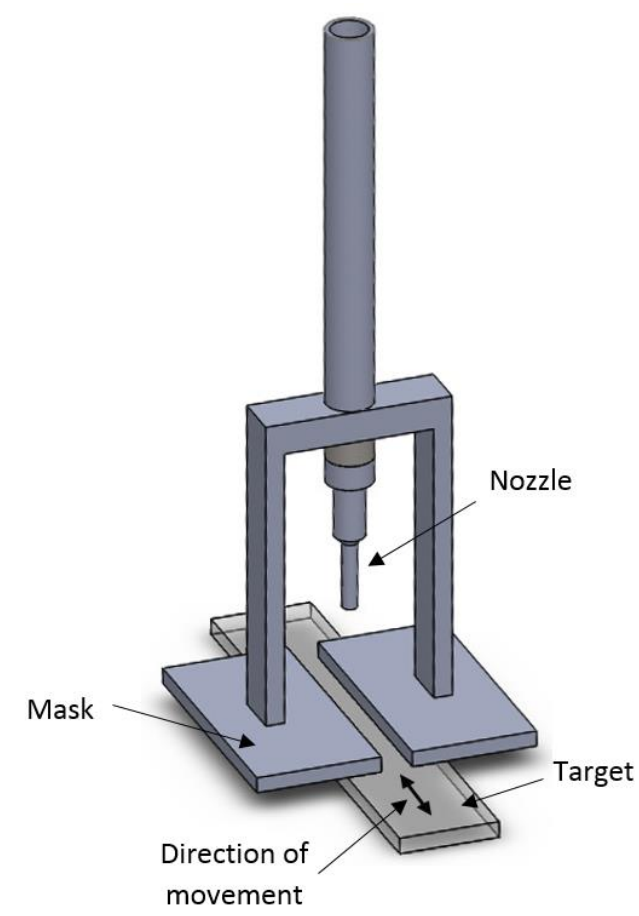

Figure 3-1 Conceptual schematic of shadow clamp of Chapter 2

\subsubsection{Measurement of shadow mask erosion}

Chapter 2 utilized the shadow mask to machine straight channels, which exposed the mild steel mask to relatively low particle doses. The oscillating target technique that was utilized in the present work, however, exposed the mask to much higher particle doses, and the mild steel masks were found to fail before the machining experiments could be completed. Previous investigators have utilized both relatively soft (e.g. copper [29]) and hard (e.g. stainless steel [34]) materials in clamped masked configurations. In an effort to determine a suitable mask material for the more 
demanding adjustable shadow mask configuration of the present work, the erosion rates of mild steel, stainless steel, tungsten carbide, copper and two samples of spring steel were measured and compared. Both the bulk erosion rates, i.e., starting from a flat surface, and the edge erosion rates, i.e., on a sharp edge, were measured. Samples of each material were cut into $1.2 \mathrm{~cm}$ by $2 \mathrm{~cm}$ specimens, and their edges were ground successively with 180 grit, 400 and 1200 grit grinding paper, using both belt and wheel grinders to ensure a sharp edge. Both the initial edge and flat profiles of each sample were measured using an optical profilometer (Nanovea ST400 Micro Photonics Inc., Irvine, CA, USA) which had a depth resolution of $25 \mathrm{~nm}$ and a lateral resolution of $0.1 \mu \mathrm{m}$. The Rockwell hardness of each specimen (Table 1) was also measured using a microhardness tester (Model LR-3E, Leco Corporation, Michigan, USA).

Table 3-1 Specimen Rockwell hardness

\begin{tabular}{ccc} 
Specimen & Indenter Type & Hardness \\
\hline Spring steel 1 (AISI 1095) & C & 90.65 \\
Spring steel 2 (AISI 1095) & C & 89.6 \\
Mild steel (AISI 302) & B & 83.6 \\
Stainless steel (AISI 316) & C & 79.85 \\
Copper (ASTM B187) & F & 66.85 \\
Tungsten carbide (C2) & C & 96.8
\end{tabular}

To reduce experimental variations associated with fluctuations in abrasive mass flow rate, the specimens were positioned side-by-side and machined without interrupting the abrasive flow. For the edge erosion tests, a rectangular nozzle $(0.2 \mathrm{~mm}$ by $3.8 \mathrm{~mm})$ was used to blast the edges of the specimens using 96 passes at a scan speed of $1 \mathrm{~mm} / \mathrm{s}$. A visual assessment of the symmetry of the resulting blast footprint ensured alignment with the edges. All samples were levelled to ensure a constant nozzle to target standoff distance of $20 \mathrm{~mm}$. Two samples of spring steel were placed at either end of the arrangement so that their erosion rates could be compared in order to assess the uniformity of the test. For the bulk erosion tests, a $1.5 \mathrm{~mm}$ diameter circular nozzle was used to blast the center (i.e. off of the edges) of the samples using 24 passes with a scan speed of $1 \mathrm{~mm} / \mathrm{s}$. 
The erosion rate $E R$ (volume of material removed per unit mass of incident abrasive) was calculated as

$$
E R=\frac{A_{c} v}{\dot{m} n}
$$

where $A_{c}$ is the cross-sectional area of material removed measured using the optical profilometer (Nanovea ST400 Micro Photonics Inc., Irvine, CA, USA), $\dot{m}$ is the particle mass flow rate, $v$ is the scan speed, and $n$ is the number of passes of the jet. Each erosion experiment was repeated two times, and for each resulting channel, five profiles were measured.

\subsubsection{Micro-machining experiments using oscillating target}

Trapezoidal, U, and $\mathrm{W}$-shaped channels were created by using the computer controlled stages to rapidly oscillate the target sideways ( $X$ direction in Figure 3-2) with an amplitude, $A$ between $100 \mu \mathrm{m}$ and $600 \mu \mathrm{m}$ at frequencies $\omega$, between $1 \mathrm{~Hz}$ and $15 \mathrm{~Hz}$, while simultaneously scanning linearly in the $Y$ direction (Figure 3-2) at scan speeds $v_{s}$, ranging between $1 \mathrm{~mm} / \mathrm{s}$ and 5 $\mathrm{mm} / \mathrm{s}$. Either the stationary nozzle at a $100 \mu \mathrm{m}$ standoff, or the contact shadow mask system with a mask opening of $150 \mu \mathrm{m}$ to $450 \mu \mathrm{m}$ (Figure 3-1), were used. The mass flow rate at the nozzle exit for the maskless (small round nozzle) and shadow mask techniques (larger rectangular nozzle) were $2.8 \mathrm{~g} / \mathrm{min}$ and $5.6 \mathrm{~g} / \mathrm{min}$ respectively. Multiple passes using the oscillating target were used to create deeper channels. Each reported profile represented the average of 15 data sets, i.e., three separately repeated repetitions and five profiles along the length of each channel. The reported profile was that which had a depth closest to the average depth of these. The maximum difference in depths for the profiles within in a given channel, and between repeats of different channels were less than 5\% and $10 \%$ respectively. Table 3-2 shows a summary of the experimental conditions used in the work.

Although it was possible to create a near uniform erosive efficacy at the center of the resulting feature where the lateral $X$ direction velocity was approximately constant, at each end of the oscillation scan, the stage decelerated, stopped and accelerated in the opposite direction. The periphery of the channel machined in this decelerating region was thus exposed to a higher erosive 
efficacy than the center, giving rise to a $\mathrm{W}$-shaped channel. Depending on the amplitude, and using post-processing passes, $\mathrm{U}$ and trapezoidal shaped micro-channels could also be fabricated.

Table 3-2 Process parameters used in experiments

\begin{tabular}{|c|c|c|c|c|c|c|}
\hline Experiment & $A(\mu \mathrm{m})$ & $\omega(\mathrm{Hz})$ & $\begin{array}{c}v_{s} \\
(\mathrm{~mm} / \mathrm{s})\end{array}$ & Nozzle & Technique & Material \\
\hline Effect of amplitude & $0-250$ & 10 & 2 & blue & $\begin{array}{c}\text { Shadow } \\
\text { mask }\end{array}$ & Glass \\
\hline Effect of foot print & 250 & 15 & 1 & blue & $\begin{array}{l}\text { Shadow } \\
\text { mask }\end{array}$ & Glass \\
\hline Glass model comparison & 320 & 15 & 1 & blue & $\begin{array}{l}\text { Shadow } \\
\text { mask }\end{array}$ & glass \\
\hline $\begin{array}{l}\text { PMMA model } \\
\text { comparison }\end{array}$ & 320 & 15 & 1 & purple & maskless & PMMA \\
\hline $\begin{array}{c}\text { Trapezoidal shaped } \\
\text { channel }\end{array}$ & $\begin{array}{c}300 \text { and } \\
0\end{array}$ & 15 & 1 and 3.5 & purple & maskless & glass \\
\hline
\end{tabular}

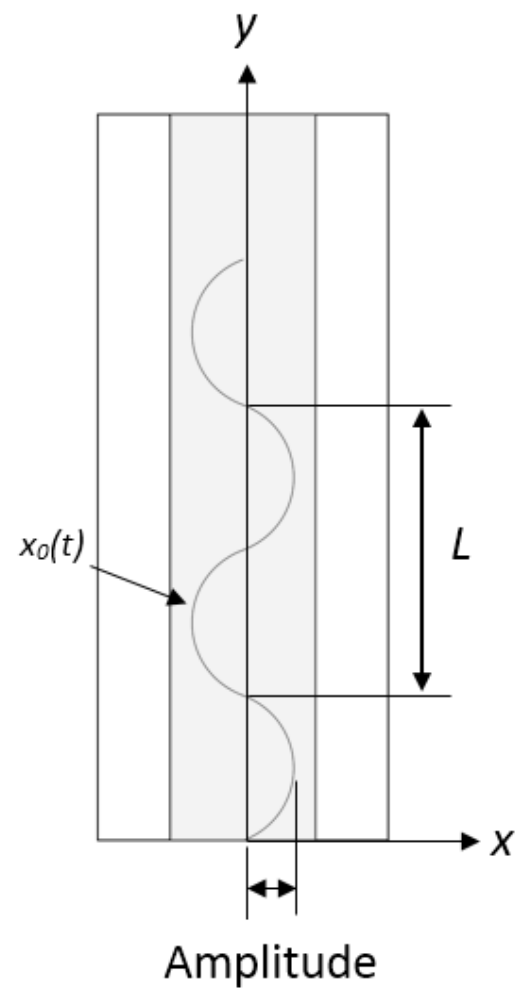

Figure 3-2 Schematic of an oscillating pass. $L$ is the lead, which when divided by the scan speed, gave the frequency. The location of the center of the source relative to the oscillating target is described by $x_{0}(t)$. 
In order to verify that the shape, amplitude and frequency of the sinusoidal oscillation produced by programming the computer controlled stages was reproduced faithfully in the machining path, the oscillation path was measured as the scar left by a machining experiment performed with a very high $Y$-direction scan speed. A comparison between the scar and a superimposed sinusoidal function having the same parameters revealed a negligible difference.

\subsection{Modeling}

\subsubsection{Surface evolution equation}

Slikkerveer et al. [36] and ten Thije Boonkkamp and Jansen [35] were the first to develop an analytical surface evolution model based on local erosion rate for brittle materials. The models were refined in subsequent work, leading to the following expression for the surface evolution of two-dimensional channel (Figure 3-3) by a non-oscillating abrasive jet [3]:

$$
z_{, t^{*}}^{*}=(1-\varepsilon \kappa) E^{*}\left(x^{*}\right)\left(1+z_{, x^{*}}^{* 2}\right)^{-k / 2}
$$

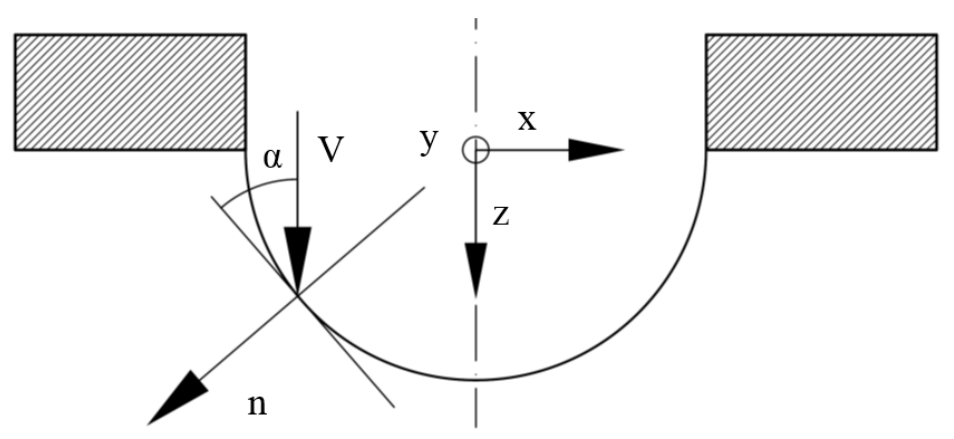

Figure 3-3 Schematic of coordinates used for masked channel cross-sectional profiles.

where $z^{*}=z / L, x^{*}=x / L$ and $t^{*}=t / T$ are the non-dimensional depth, channel width and time, respectively, and $\rho_{s}$ is the density of the target material. The use of commas and subscripts indicates partial differentiation with respect to the subscript, e.g. $z^{*}{ }_{t}{ }^{*}=\partial z^{*} / \partial t^{*}$. The normalized erosive efficacy, $E^{*}$, characterizes the potential of a jet to erode a surface [39], 


$$
E^{*}=\varphi^{*}\left(x^{*}\right)\left[V^{*}\left(x^{*}\right)\right]^{k}
$$

A time constant, $T$, was defined as the time required to propagate the surface at $x=0$ over a characteristic length, $L$, in the $z$ direction such that $T=\left(L \rho_{s} / C V(0)^{k} \varphi(0)\right)$ [64]. The appropriate value of characteristic length is discussed in Section 3.3.2. The particle mass flux and velocity were normalized by their values at $x=0$, i.e. $\varphi^{*}\left(x^{*}\right)=\varphi\left(x^{*}\right) / \varphi(0)$ and $V^{*}\left(x^{*}\right)=$ $V\left(x^{*}\right) / V(0)$, and $C$ and $k$ are experimentally determined erosion rate constants related to particle and target characteristics. The first term in Eq. (3.2) provides viscous smoothing to ensure the solution remains stable, and the curvature $\kappa=z_{, x^{*} x^{*}}^{*} /\left(1+z_{, x^{*}}^{*}\right)^{3 / 2}$ in which $\varepsilon$ is a constant smoothing factor determined using the guidelines provided by Ghobeity et al. [43].

For ductile materials Ghobeity et al. [39] showed that the normalized surface evolution model is

$$
z_{, t^{*}}^{*}=(1-\varepsilon \kappa) E^{*}\left(x^{*}\right) \sqrt{\left(1+z_{, x^{*}}^{* 2}\right)} g(\alpha)
$$

The empirical function $g(\alpha)$ describes the impact angle dependence of the erosion rate and is usually expressed in the form suggested by Oka et al. $[18,19,65]$

$$
g(\alpha)=(\sin \alpha)^{n_{1}}\left[1+H_{v}(1-\sin \alpha)\right]^{n_{2}}
$$

where the constants $n_{1}$ and $n_{2}$ are curve fitted to erosion rate data and depend on the particle hardness and other impact conditions, while $H_{v}$ is the initial target hardness in GPa [18,19]. The angle between the tangent to the channel surface in the $x-z$ plane and the $z$ component of the incident velocity vector, $\alpha$ (Figure 3-3), is given by [37]

$$
\alpha=\left[\frac{\pi}{2}-\arccos \left(\frac{1}{\sqrt{1+z_{, x^{*}}^{* 2}}}\right)\right]
$$


For $\varphi^{*}=1$ and $V^{*}=1$ everywhere, the predicted feature cross section would be flat. However, in the presence of a mask or, as in this work, a moving mask or moving nozzle, the erosive efficacy function $E^{*}$ must be determined.

\subsubsection{Determination of erosive efficacy}

The aim is to use a measured shallow straight channel profile, $z_{N}(x)$ made by scanning in the $Y$ direction without oscillating to infer the erosive efficacy delivered to the surface when it oscillates. It is assumed a sufficiently low oscillation frequency is used to not introduce significant waviness along the length of the channel. It will first be demonstrated that the erosive efficacy from scanning a nozzle over a given channel length is directly related to that delivered by an imagined stationary source whose length is equal to the channel length. Then the erosive efficacy delivered by the imagined stationary source as it oscillates will be derived.

If $\varphi_{N}(x, y)$ is the particle flux $\left(\mathrm{kg} /\left(\mathrm{m}^{2} \mathrm{~s}\right)\right)$ delivered to the surface by a nozzle that leaves an eroded footprint of length $L_{f}$ shown in Figure 3-4, then, for a straight scan of such a nozzle in the $+y$ direction, the location of the nozzle at any time is $y=L_{f}-v_{s} t$, where $v_{s}$ is the nozzle scan speed. This produces a nozzle erosive efficacy, $E_{N}(x, t)=C v^{k} \varphi_{N}\left(x, L_{f}-v_{s} t\right)$ where $C v^{k}$ is the volumetric erosion rate $\left(\mathrm{m}^{3} / \mathrm{kg}\right)$, i.e. the volume of target material removed per unit mass of incident particles [36], in which $C$ is a constant and $v$ is the particle velocity.

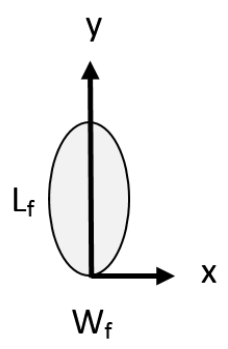

Figure 3-4 Footprint left by the nozzle.

Following the arguments presented by Ghobeity et al. [39], if the resulting $z$ profile is shallow ( zero slope) the dimensional version of the surface evolution Eqs. (3.2) and (3.3) reduce to 


$$
\frac{\partial z_{n}(x, t)}{\partial t}=E_{N}\left(x, L_{f}-v_{s} t\right)
$$

The cross-sectional profile of a shallow channel resulting from a scan over the footprint length $L_{f}$ is

$$
z_{N}(x)=\int_{0}^{L_{f} / v_{s}} E_{N}\left(x, L_{f}-v_{s} t\right) d t
$$

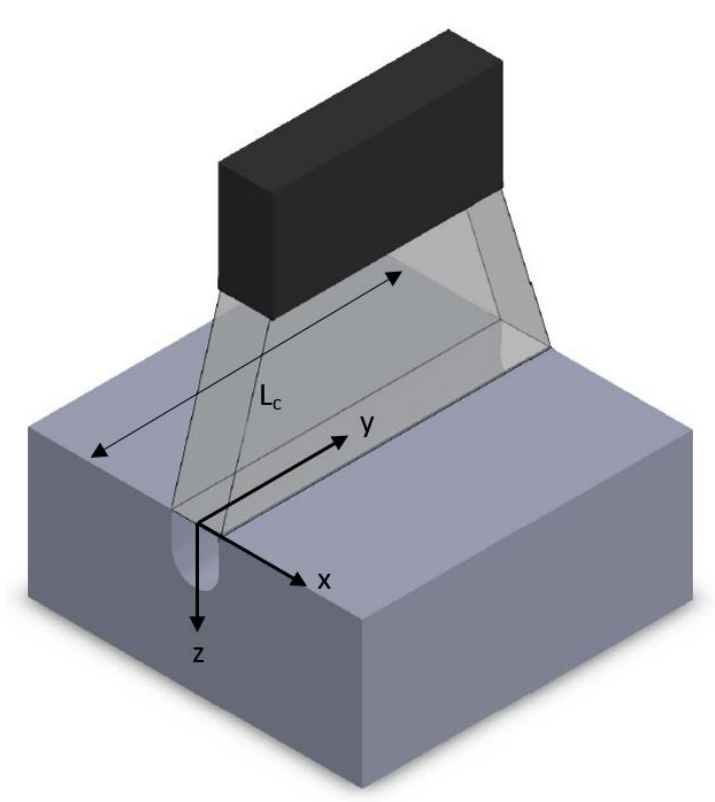

Figure 3-5 Coordinate system for a channel resulting from a stationary erosive efficacy source $E_{L}$, of length $L_{c}$

One now imagines a stationary erosive efficacy source that delivers an identical flux $\varphi_{L}(x)$ to every $y$ location along the length of a channel $L_{c}$ (Figure 3-5). If the associated erosive efficacy $E_{L}(x)=C v^{k} \varphi_{L}(x)$ is delivered for a time $t$ to the entire length of a channel of length $L_{c}$, the shallow profile $z_{L}$ that would result is

$$
z_{L}(x, t)=E_{L}(x) t
$$


If the same shallow profile is assumed to result from scanning the nozzle delivering $E_{N}$ over a length of a footprint $L_{f}$ (Figure 3-4) at a scan speed $v_{s}$, as with a stationary source delivering $E_{L}$ over the length $L_{c}$ for a dwell time $t_{d}$, (Figure 3-5), then

$$
z_{L}(x)=E_{L}(x) t_{d}=z_{N}(x)=\int_{0}^{L_{f} / v_{s}} E_{N}\left(L_{f}-v_{s} t, x\right) d t
$$

Equation (3.10) implies that $E_{L}$ can be determined to within a constant $t_{d}$, by measuring the cross sectional profile $z_{N}(x)$ of a shallow channel resulting from a straight scan of the nozzle, i.e.

$$
E_{L}(x)=\frac{z_{N}(x)}{t_{d}}
$$

If it is now assumed that this source oscillates with its center at $x_{0}(t)=A \sin (2 \pi \omega t)$ (Figure 3-2), where $A$ and $\omega$ are the amplitude and frequency (Hz), then the instantaneous erosive efficacy is $E_{L}\left(x-x_{0}(t)\right)$. The dimensionless surface evolution Eqs. (3.2) and (3.4) become

$$
z_{, t^{*}}^{*}=(1-\varepsilon \kappa) z_{N}^{*}\left(x^{*}-x_{0}^{*}(t)\right)\left(1+z_{, x^{*}}^{* 2}\right)^{-k / 2}
$$

for brittle materials, and

$$
z_{, t^{*}}^{*}=(1-\varepsilon \kappa) z_{N}^{*}\left(x^{*}-x_{0}^{*}(t)\right) \sqrt{\left(1+z_{, x^{*}}^{* 2}\right)} g(\alpha)
$$

for ductile materials. In this case, $z_{0}, z_{N}, x$, and $x_{0}$ have been normalized by $z_{N}^{*}(0)$, and $t$ has been normalized by $t_{d}$. The value of $t_{d}$ is closely related to the erosion rate of the target material at perpendicular incidence which, analogous to previous work for straight channels [39], can be determined by matching the depth of the predicted and measured first pass oscillating profiles. In other words $t_{d}$ is adjusted until the depth predicted in Eq. (3.12) or (3.13) at $t_{1}{ }^{*}$ matches that measured on a shallow single pass $\mathrm{W}$-shaped channel. The profile for deeper channels resulting from $n$ oscillating passes could be determined by solving Eqns. (3.12) and (3.13) at $t^{*}=n t_{1}{ }^{*}$. 
Equations (3.12) and (3.13) involve a time-dependent erosive efficacy. An equivalent alternate formulation which eliminates this time dependency and is more useful for visualizing the erosive efficacy can be obtained as follows. The $E^{*}$ delivered to each region $d x$ on the target depends on the instantaneous source $X$-direction (i.e. oscillating direction) velocity and, as a result, the amount of time that oscillating source spent on that region. For a sinusoidal oscillation, the time $t$ at which the source center can be found at a given location $x_{0}$ is

$$
t=\frac{1}{2 \pi \omega} \sin ^{-1}\left(\frac{x_{0}}{A}\right)
$$

Within a given oscillation where $x_{0}$ varies from $-A$ to $A$ in a time $\bar{T} / 2$, the proportion of time that the source can be found between $x_{0}$ and $x_{0}+\mathrm{d} x_{0}$ is thus

$$
\frac{d t}{\bar{T} / 2}=\frac{1}{\pi A \sqrt{1-\left(\frac{x_{0}}{A}\right)^{2}}} d x_{0}
$$

where $\bar{T}=1 / \omega$ is the period of the oscillation. Since the right hand side of Eq. (3.15) can be recognized as the probability density function of the sine wave, it can be interpreted as the probability of finding the source between $x_{0}$ and $x_{0}+\mathrm{d} x_{0}$, i.e.

$$
P\left(x_{0}\right) d x_{0}=\frac{1}{\pi A \sqrt{1-\left(\frac{x_{0}}{A}\right)^{2}}} d x_{0}
$$

The change in total erosive efficacy as the source moves laterally a distance $d x_{0}$ is the erosive efficacy of the source multiplied by the probability of being between $x_{0}$ and $x_{0}+\mathrm{d} x_{0}$, i.e. :

$$
d E(x)=P\left(x_{0}\right) E_{L}\left(x-x_{0}\right) d x_{0}
$$


and the total erosive efficacy delivered to the surface in one oscillation will be:

$$
E(x)=\int_{-A}^{A} \frac{z_{N}\left(x-x_{0}\right)}{t_{d} \pi A \sqrt{1-\left(\frac{x_{0}}{A}\right)^{2}}} d x_{0}
$$

In order to use $E(x)$ in the surface evolution Eqs. (3.2) and (3.4), it was normalized by dividing it by its value at $x=0$, and all length dimensions were divided by the sum of the width of the single pass straight foot print, $W_{f}$, and $2 A$, i.e. the characteristic length $L=W_{f}+2 A$. In this scheme, a time invariant normalized erosive efficacy delivered by one oscillation is used to propagate the surface evolution, instead of the time-dependent one in Eqs. (3.12) and (3.13). The results are identical in either case.

\subsection{Results and discussion}

\subsubsection{Edge erosion for different mask materials}

Figure 3-6 shows the measured erosion rate of the edges of the various mask materials tested for use in the shadow mask system. The two spring steel samples positioned at either end of the configuration (sample 1 machined at the beginning and sample 2 at the end) were used to measure the uniformity of the test. The erosion rate of the two samples of spring steel differed by $4.5 \%$ from the average, indicating that the mass flow rate did not significantly change during the test. The erosion rates for channels machined in the middle of the samples (not shown) followed the same trend. 


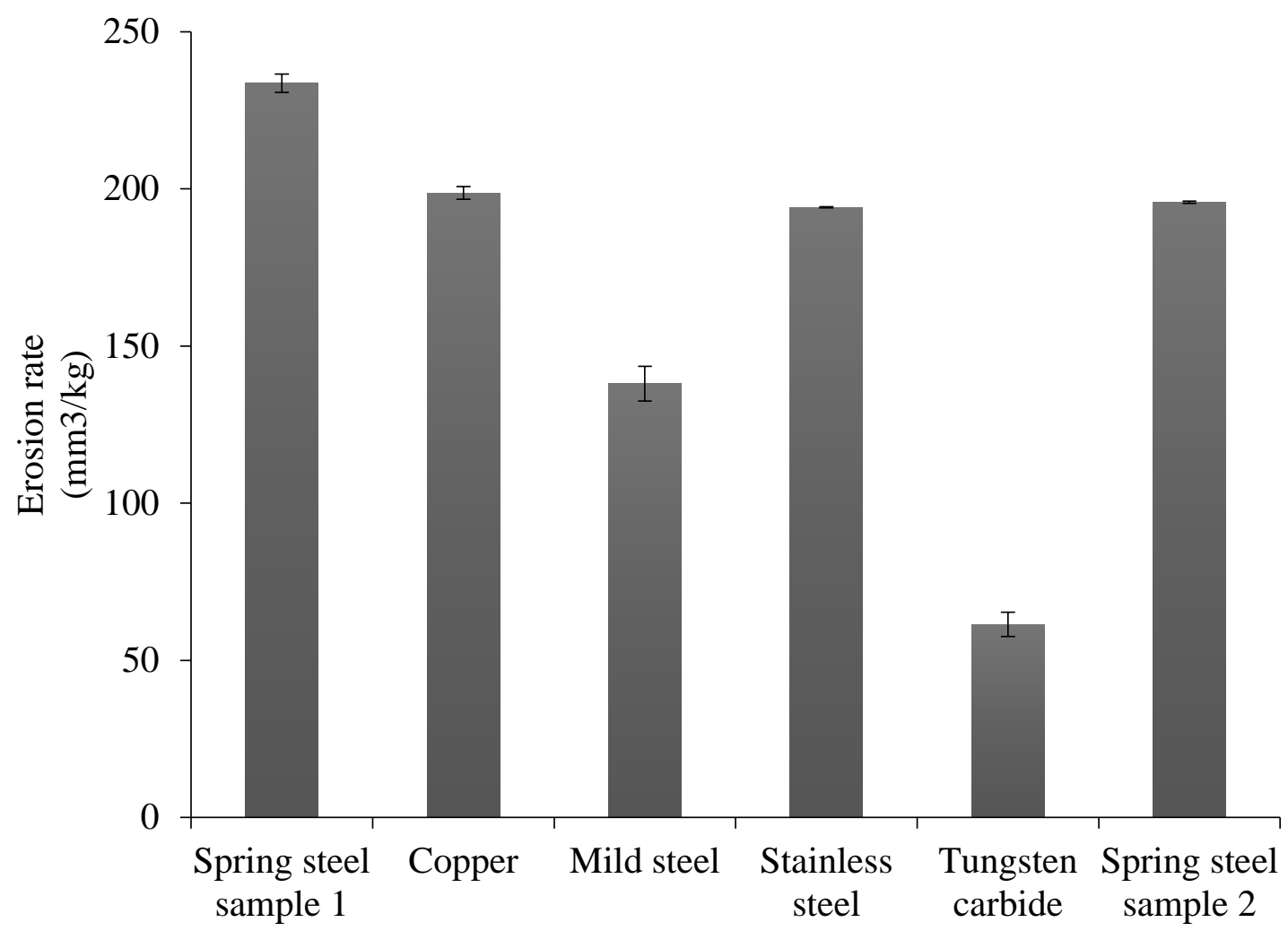

Figure 3-6 Erosion rate for different mask materials with error bars representing one standard deviation.

Figure 3-7 shows the eroded profiles of each of the tested samples. Since the local slopes are very small $\left(<2^{\circ}\right)$, there was a negligible effect of incident local angle on erosion rate, i.e. the erosion rate was measured at effectively normal incidence. The tungsten carbide and spring steel samples had the lowest and highest, respectively, erosion rates. Although the tungsten carbide was also the hardest material tested, there was, in general, no strong correlation between hardness and erosion rate. Given its much lower erosion rate, tungsten carbide was utilized in all shadow mask experiments. 


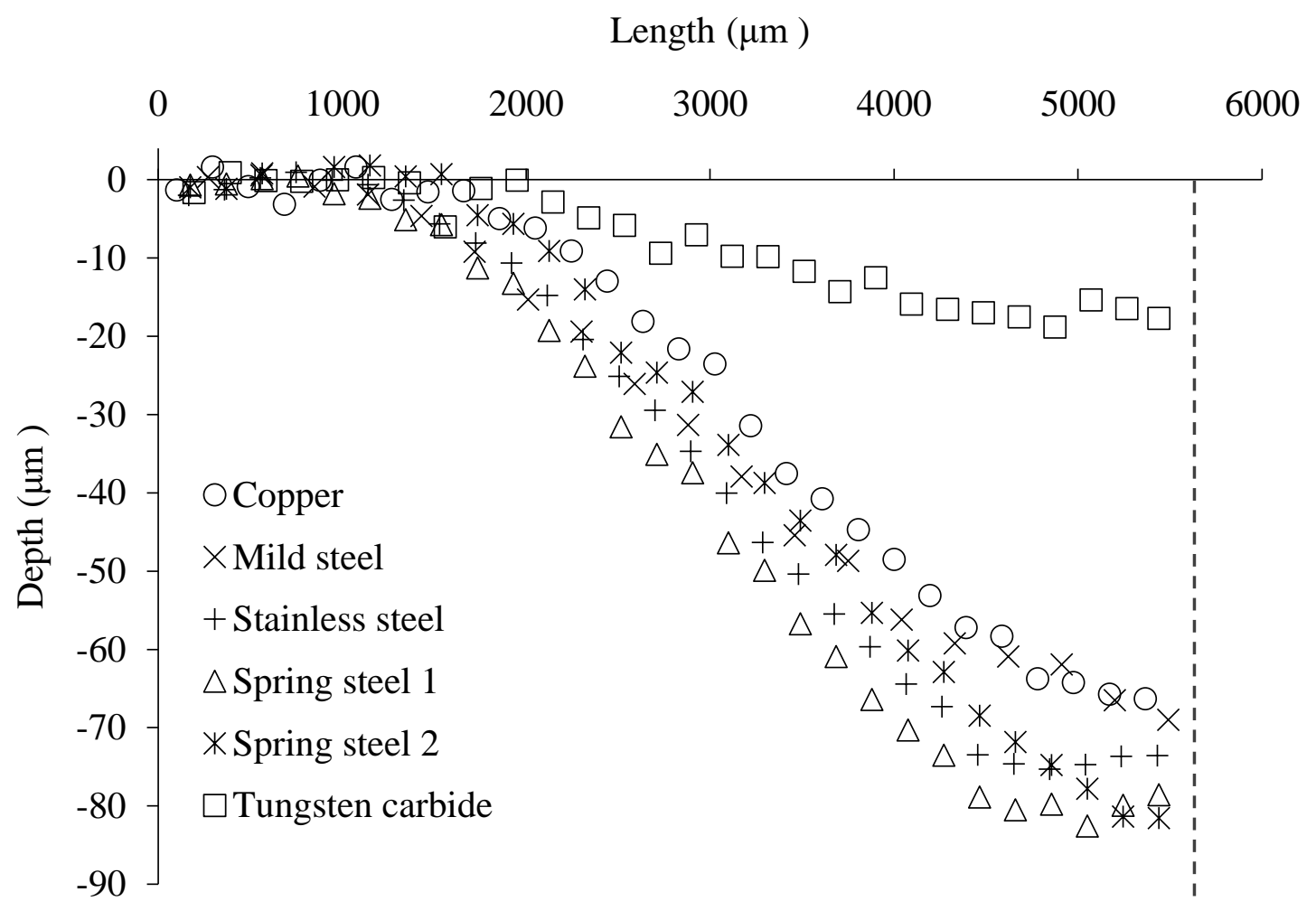

Figure 3-7 Profile of edges after erosion tests. Edge of samples prior to erosion is indicated by the dashed line.

\subsubsection{W and $V$ shaped channels}

Figure 3-8 shows a typical measured normalized non-oscillating erosive efficacy of a straight channel, i.e. the normalized Eq. (3.11), along with the distribution of erosive efficacy predicted by the model of Section 3.3.2, for an oscillation with amplitude of $400 \mu \mathrm{m}$. As expected, the oscillation caused a $W$-shaped efficacy that was much wider and shallower than the stationary source. This erosive efficacy can be used in Eqs. (3.2) and (3.4) to predict the evolving surface profile both in brittle and ductile materials. 


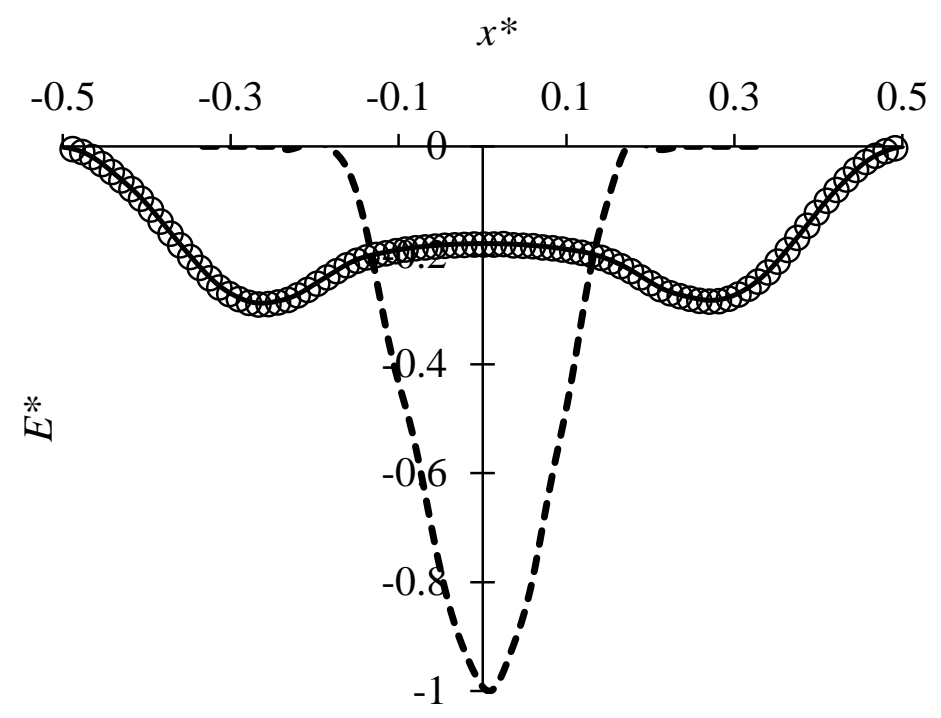

Figure 3-8 Predicted normalized erosive efficacy using the model of Section 3.3.2 (solid line, Eq. (3.10) and symbols, Eq. (3.18)). Amplitude $\mathrm{A}=400 \mu \mathrm{m}, \omega=15 \mathrm{~Hz}$. Widths, $x$, normalized by dividing by width of oscillating channel. Erosive efficacies, $E$, normalized by dividing by nonoscillating pass (dashed line) centerline erosive efficacy machined at $v_{s}=1 \mathrm{~mm} / \mathrm{s}$ using maskless technique.

\subsubsection{Glass target}

The effects of oscillation amplitude, scan speed, frequency and mask opening width on the resulting channel cross sectional shape were investigated experimentally and with the aid of the models of Section 3.3.2.

\subsection{Effect of amplitude}

Figure 3-9 shows that, for a shadow mask opening of $156 \mu \mathrm{m}$ and a frequency of $10 \mathrm{~Hz}$, as the amplitude was increased, the width of relatively shallow channels increased, and the depth decreased. This was expected since increases in amplitude imply a larger lateral ( $Y$-direction) scan distance, and, for the same scan speed, a reduced particle dose to the center of the surface. For small amplitudes, the channels were more V-shaped and as the amplitude increased, the shape of channels became more W-shaped. This was also expected since, for sufficiently small amplitudes, the erosive efficacy approaches that of the non-oscillating case. The amplitude of transition from the $\mathrm{V}$ to $\mathrm{W}$-shaped profiles depended on the size of the footprint created using either the shadow mask or maskless technique. The model predicted the transition at an amplitude $A$ between 0.75 
and 1 times of the footprint width produced by a stationary nozzle or shadow mask. For instance, increasing the oscillation amplitude above $200 \mu \mathrm{m}$ for a $156 \mu \mathrm{m}$ mask opening having a foot print width of $227 \mu \mathrm{m}$ resulted in the $\mathrm{W}$-shape, due to the deceleration of the stage at the ends of the lateral traverses which caused a larger erosive efficacy to be delivered to the periphery of the channels.

To determine the predicted profiles shown in Figure 3-9, the measured profile of a straight single pass shallow channel, $z_{N}(x)$, was measured and curve fit with a spline. It was then used in Eq. (3.12), which was solved numerically using the partial differential equation (PDE) solver (method of lines) in Mathcad 15 (PTC Inc. Needham, MA, USA). The initial condition of the problem is equation of flat surface, $z(x, 0)=0$. By comparing successively finer grids, it was found that use of $\Delta t=100$ and $\Delta x=500$, time and space steps, respectively was sufficient to ensure convergence of the numerical solution. The smoothing factor was taken as $\varepsilon=0.1$, following the suggestions of Ref. [43]. The model was able to accurately predict the shape at all amplitudes, with the exception of $\mathrm{A}=150 \mu \mathrm{m}$, which was at the temporary (i.e., a little deeper and the W-shape would disappear) transition from a $\mathrm{W}$ to $\mathrm{V}$-shaped channel.

Figure 3-10 shows a comparison between a number of predicted $\mathrm{W}$-shaped channels having the same maximum depth, using the shallow straight pass of Figure 3-9 $(A=0 \mu \mathrm{m})$. For the same depth, a decrease in amplitude led to a decrease in both the relative height and width of the central peak in the middle of the channel. This can be related to the fact that for the same frequency, the lateral scan velocity, and therefore the delivered erosive efficacy, at the center is higher if $A$ is higher. 


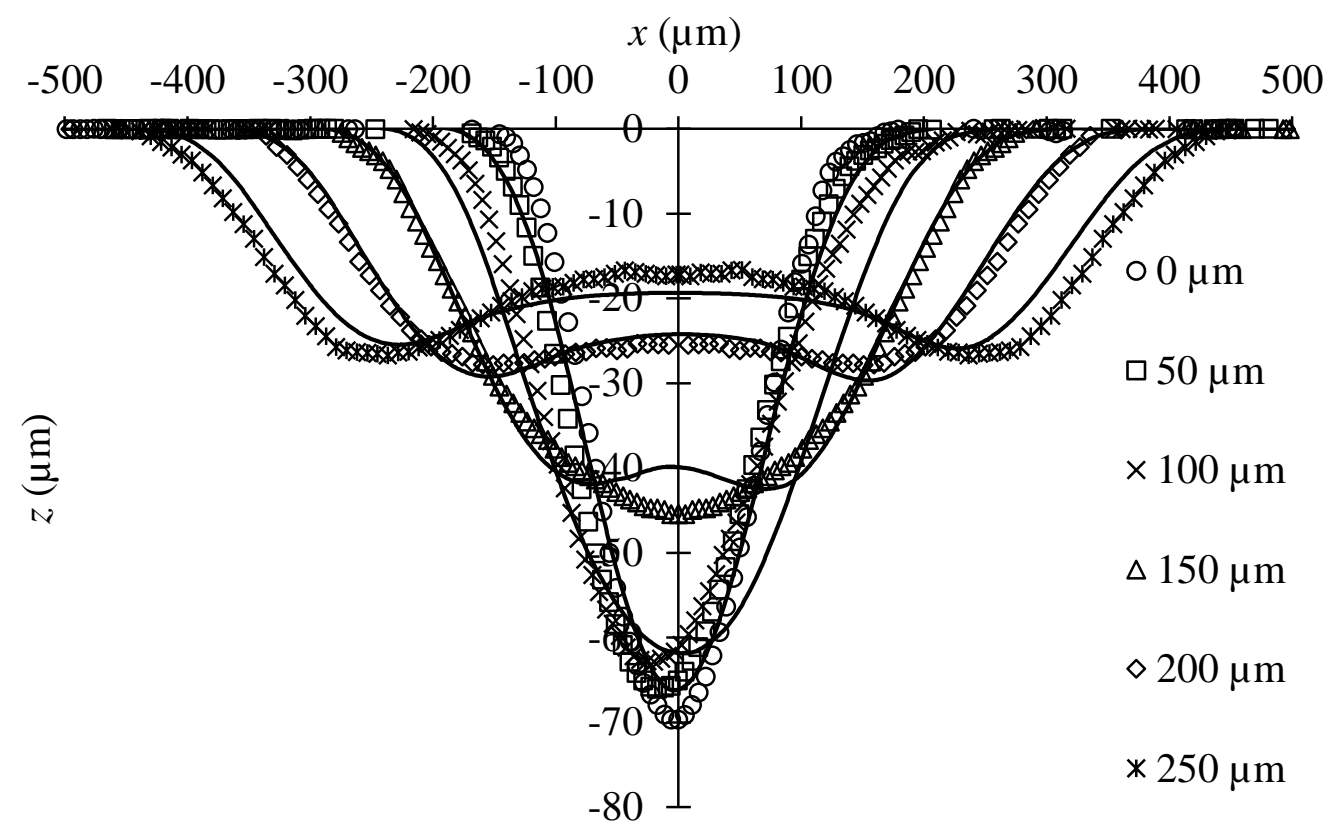

Figure 3-9 Effect of amplitude on measured (symbols) and predicted (lines) microchannel profiles made using oscillation on glass. A shadow mask opening of $156 \mu \mathrm{m}, \omega=10 \mathrm{~Hz}$, $v_{s}=2 \mathrm{~mm} / \mathrm{s}$, and 2 passes were used. The circles for $\mathrm{A}=0$ represent the non-oscillating pass.

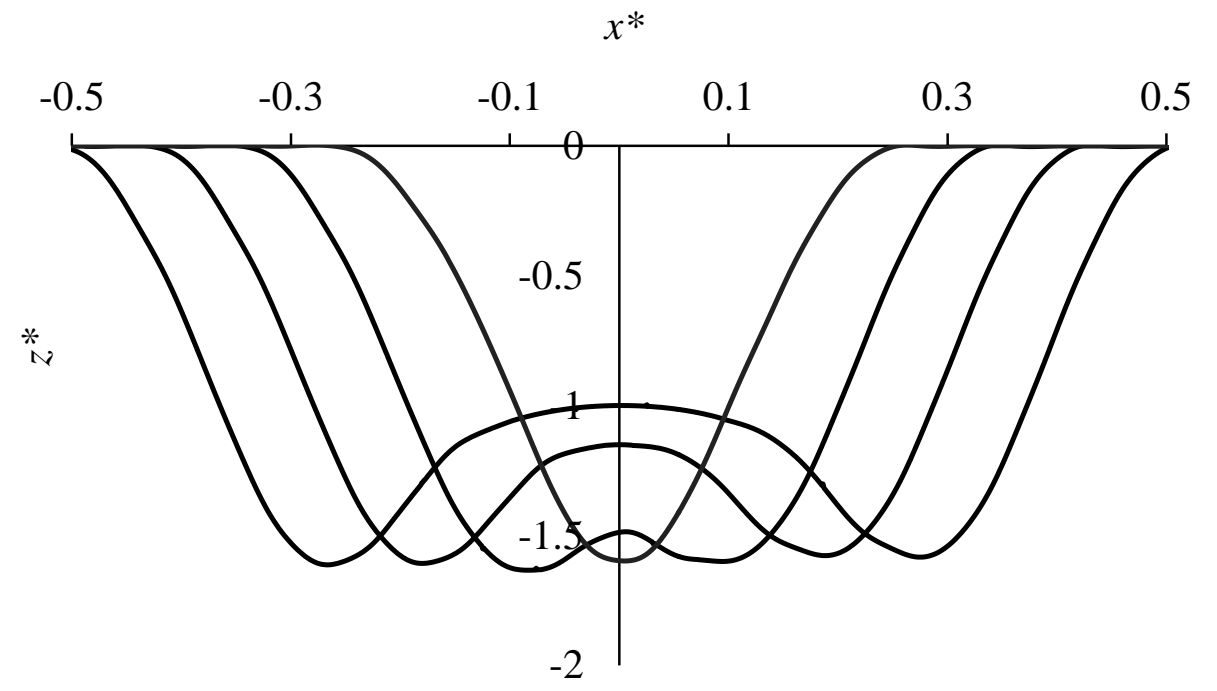

Figure 3-10 Effect of amplitude on channel shape for $A=100,200,300$ and $400 \mu \mathrm{m}$ and $\omega=15$ $\mathrm{Hz}$. Width $\mathrm{x}$, and depth $z$, normalized by dividing by $2 A+W_{f}$, and $E(x=0)$ of widest channel, respectively. 


\subsection{Effect of scan speed and frequency}

The scan speed in the $y$ direction regulated the total erosive efficacy seen by the surface, and thus the overall depth. It was found that using scan speeds between 1 and $5 \mathrm{~mm} / \mathrm{s}$, while adjusting the number of passes to keep the overall machining time constant, did not significantly change the profile shape. By maintaining the frequency above a threshold value, $\omega_{t}$, the channel shape was found to be independent of the frequency. The threshold depended on the scan speed as well as the footprint of the nozzle or mask opening, with higher scan speeds requiring higher threshold frequencies and larger footprints requiring smaller threshold frequencies. Figure 3-11 shows the overlap of footprints in a half cycle of a sinusoidal traverse, with the centerline having moved a distance $d_{f}$ in the $y$ direction in this time.

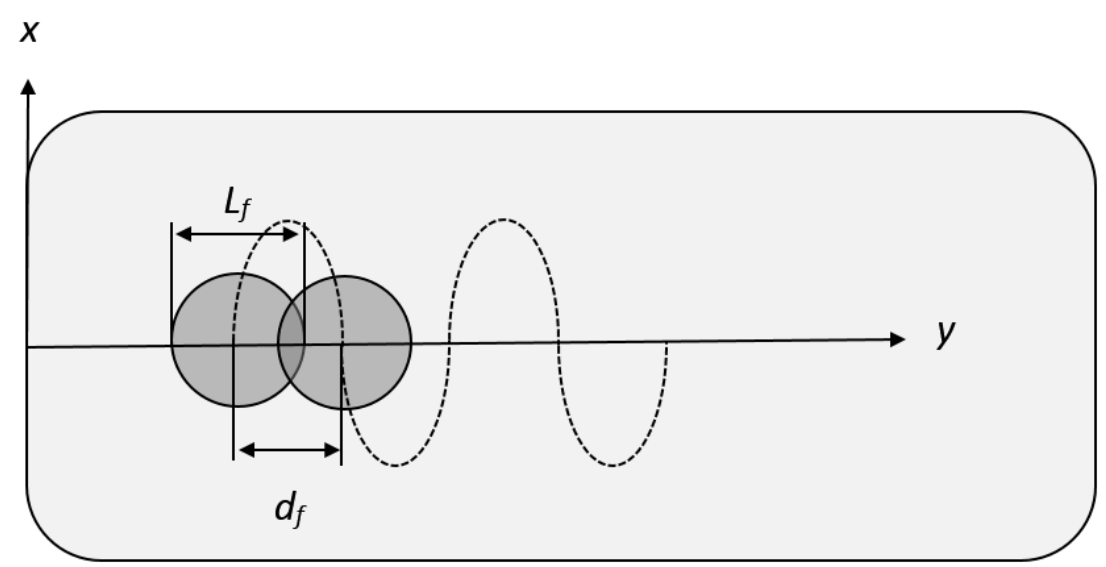

Figure 3-11 Schematic of foot prints of nozzle at the beginning and at half a period time span; the dashed line shows the path of oscillation.

A lower $d_{f}$ implies a greater degree of overlapping between the two foot prints which consequently results in lower waviness along the length of the channel. If the length travelled $d_{f}=$ $v_{s} / \omega$ is assumed to be equal the length of the footprint, i.e. $d_{f}=L_{f}$ (Figure 3-4), then the minimum required frequency to prevent significant waviness, or in other words prevent the two completely separate footprints, can be found as

$$
\omega_{t}=\frac{v_{s}}{L_{f}}
$$


For $v_{s}=2 \mathrm{~mm} / \mathrm{s}$ and a measured footprint length $L_{f}=227 \mu \mathrm{m}, \omega_{t}$ was measured as $9 \mathrm{~Hz}$, which agrees with the $8.83 \mathrm{~Hz}$ found using Eq. (3.19). The resulting measured waviness at $9 \mathrm{~Hz}$ was $0.35 \mu \mathrm{m}$, as opposed to, e.g. $0.68 \mu \mathrm{m}$ for $1 \mathrm{~Hz}$. Since the waviness of a non-oscillating straight channel was of the same order, i.e. between 0.3 and $0.4 \mu \mathrm{m}$, the threshold criteria was deemed to be acceptable. All further experiments were conducted with $\omega>\omega_{t}$. Figure 3-12 shows a typical "W" shape profile machined in this manner using an amplitude of $250 \mu \mathrm{m}$, scan speed of $2 \mathrm{~mm} / \mathrm{s}$ and frequency of $9 \mathrm{~Hz}$. The difference in depth between Figure 3-12 and Figure 3-9 was due to different abrasive flow rates and number of passes.

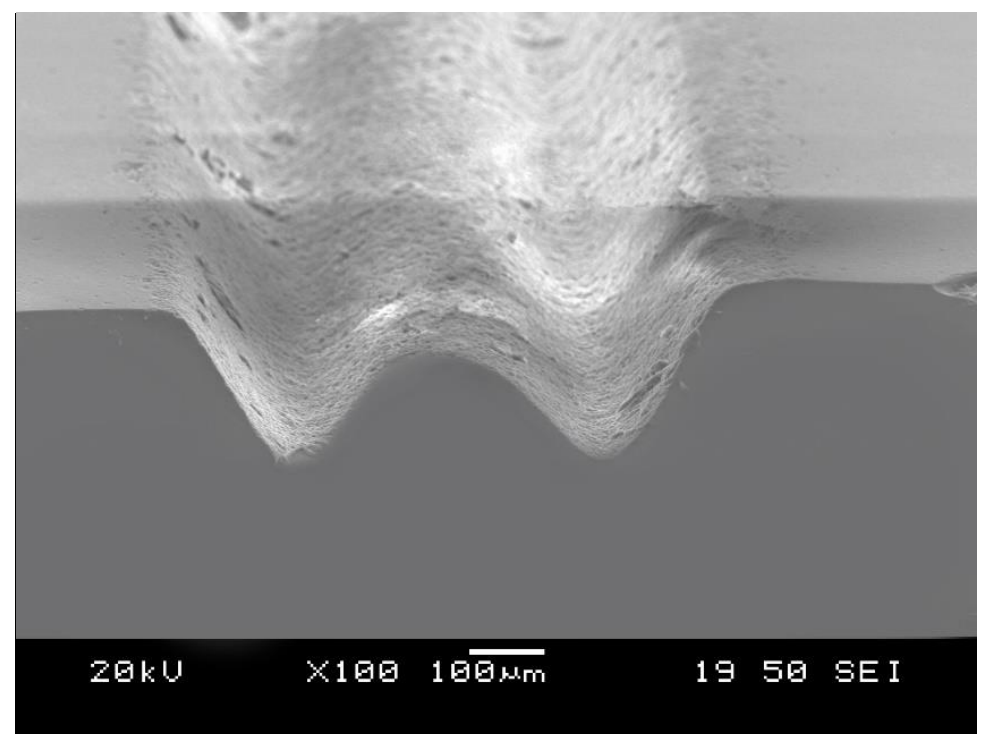

Figure 3-12 A typical "W" shape profile machined in glass made by oscillation of the target below the shadow mask setup. Experimental conditions; mask opening $=156 \mu \mathrm{m}, A=250 \mu \mathrm{m}$, $v_{s}=2 \mathrm{~mm} / \mathrm{s}$ and $\omega=9 \mathrm{~Hz}$. The darker horizontal line a distance behind the front edge is an artifact of the SEM process.

\subsection{Effect of erosive efficacy footprint width}

Figure 3-13 and Figure 3-14 show the effect of the shadow mask opening width on nonoscillating and oscillating channel profiles. Increases in mask opening (i.e. footprint width) increased the non-oscillating channel depth since it delayed the commonly observed decreased erosion resulting from the 'blast lag effect' that results when the two side walls meet at the center [63]. The increase of depth with increase in mask opening in the oscillating channel was due to the overall increase in erosive efficacy. 


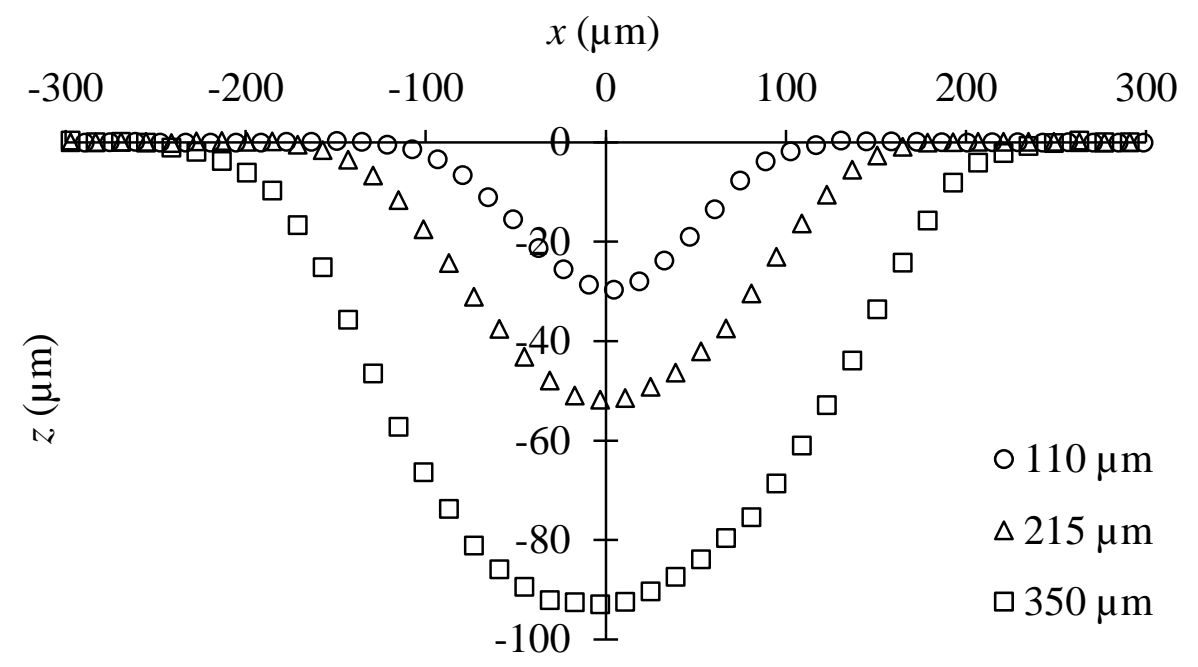

Figure 3-13 Effect of mask opening on non-oscillating channels (two passes machined at $v_{s}=1$ $\mathrm{mm} / \mathrm{s})$.

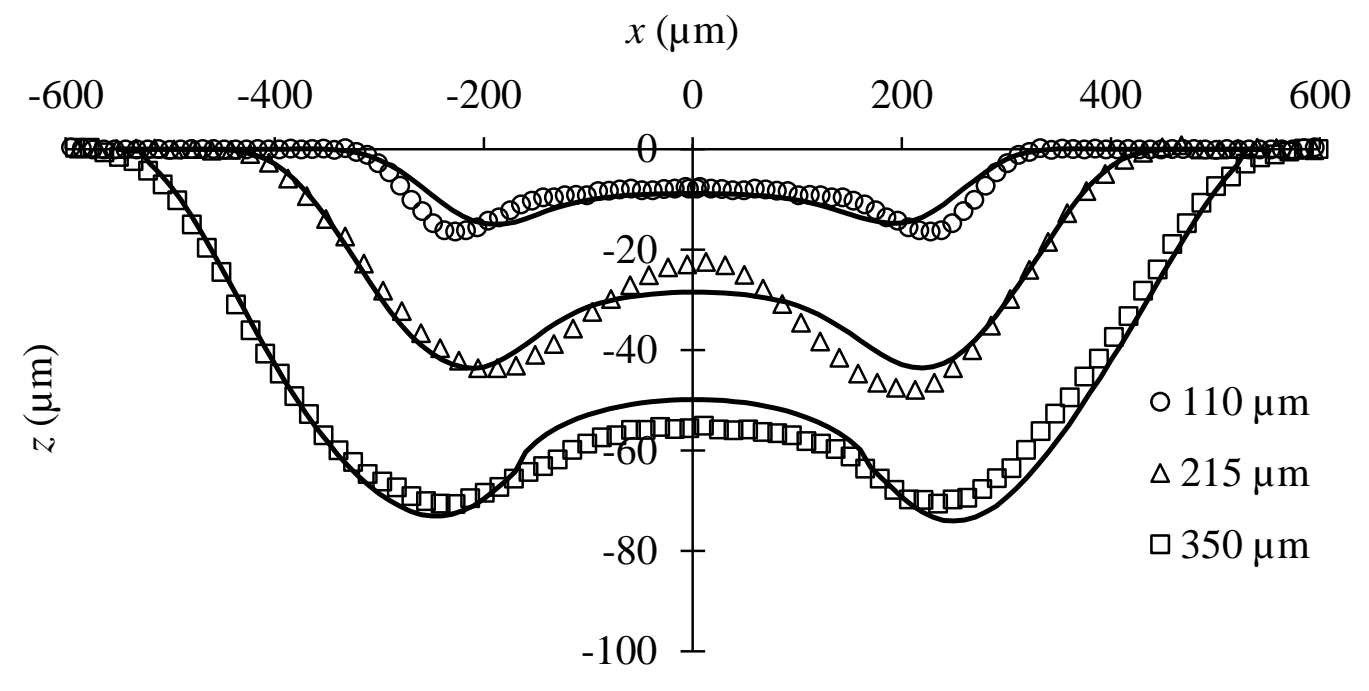

Figure 3-14 Predicted (lines) and measured (symbols) micro-channel profiles machined using different mask openings for 4 passes at $v_{s}=1 \mathrm{~mm} / \mathrm{s}$ with target oscillating at $\omega=15 \mathrm{~Hz}$, and $A=$ 250. Symbols are the experimental results and solid lines are the results from the model.

\subsection{Prediction of surface evolution}

Figure 3-15 shows the measured and predicted profiles of channels made using oscillation with multiple passes. The predictions were made in the same manner as described in Section 
3.4.2.1.1. For multiple passes, the predictions were made by scaling the time at which the solution of the surface evolution was evaluated by the number of passes. For channels below aspect ratio (ratio of depth to width) of 0.35 , the channels had a "W" shape. After that, a flat middle area began to appear which was quickly eroded because, for brittle materials, the maximum erosion occurs at normal incidence, leading to a much larger erosion rate on the flat center than the sloped sidewalls.

The predicted profiles are in very good agreement with measured ones, both in depth and shape up to an aspect ratio of approximately 0.5 (max depth difference $<8 \%$ ). Beyond this, second strike effects that resulted from ricocheted particles from the channel side-walls into the center of the channel resulted in an approximately 10\% under-prediction of depth. This well-known phenomenon causes an increase in the erosion rate at the channel center that cannot be modelled without advanced particle-tracking methods [66].

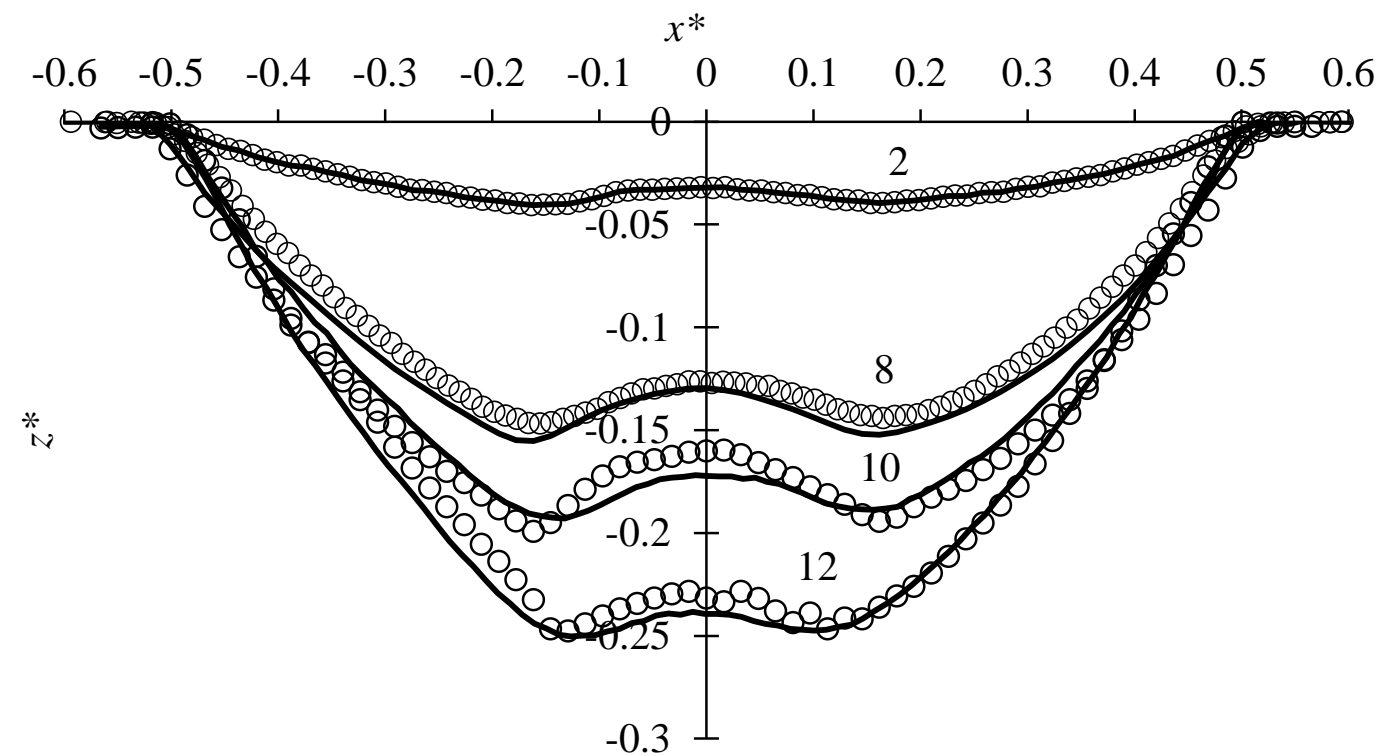

(a) 


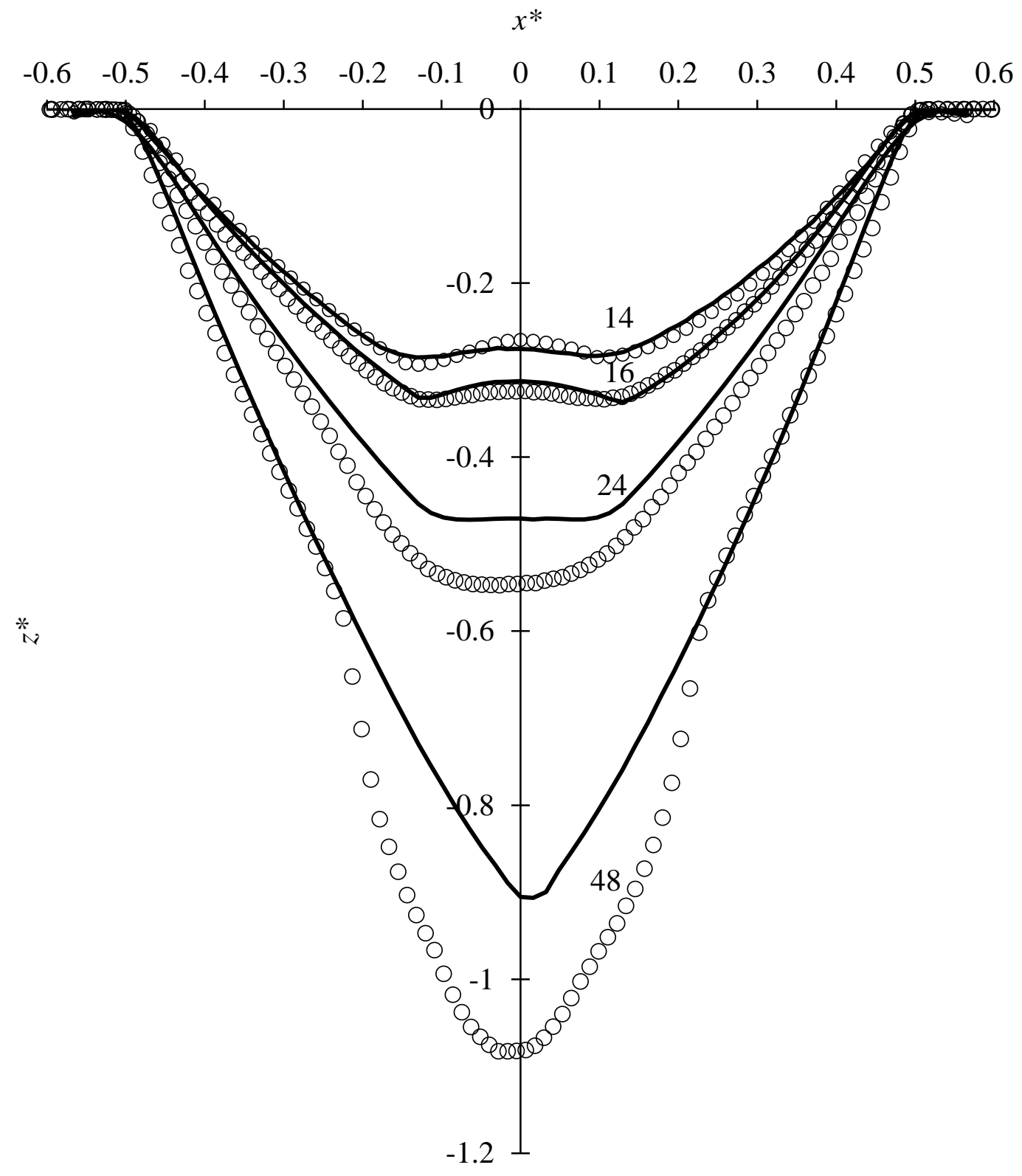

(b)

Figure 3-15 Comparison of measured (symbols) and predicted (lines) micro-channel crosssectional profiles machined using oscillation with $A=320 \mu \mathrm{m}, v_{s}=1 \mathrm{~mm} / \mathrm{s}$ and $\omega=15 \mathrm{~Hz}$ in glass using the shadow mask with opening of $410 \mu \mathrm{m}$. The depth and width are normalized by $2 A+W_{f}$ $=1237 \mu \mathrm{m}$. Number of passes is shown on each line. (a) shallow channels; (b) deeper channels. 
Both the maskless and shadow mask techniques resulted in some frosting of the surface adjacent to the channel edges. The frosted region was measured following the method presented in Chapter 2 and found to be no more than $50 \mu \mathrm{m}$ for the shadow mask and no more than $70 \mu \mathrm{m}$ for the maskless technique (Figure 3-16).

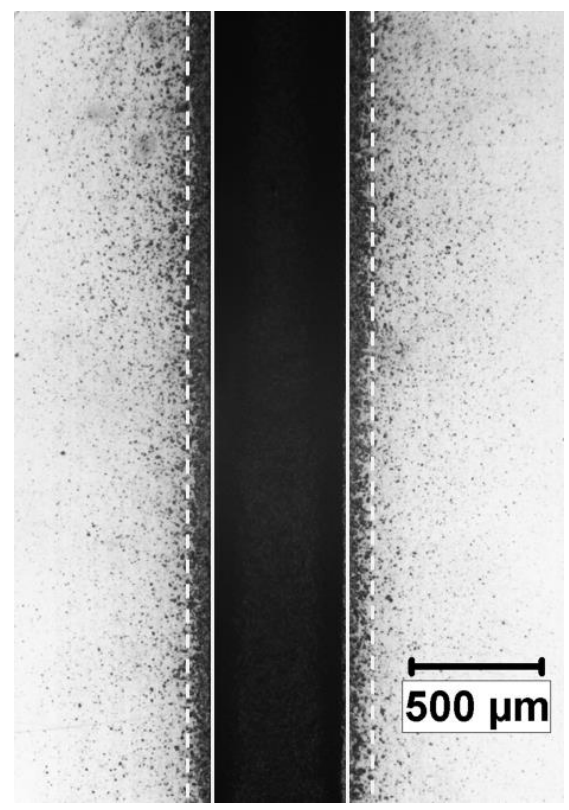

Figure 3-16 Edge frosting for a typical W-shaped channel. Solid lines show the edge of the channel and the dashed lines show the edge of the frosted area.

\subsubsection{PMMA (ductile material)}

The W-shaped channels made in PMMA were shaped differently from those machined in glass. The side walls were steeper and the profile was more curved in the deepest parts, compared to those in glass. However, the overall trends with respect to the effect of frequency, amplitude, footprint size, and scan speed on the waviness, width and depth were much the same as in glass and are thus not repeated here. Figure 3-17 presents examples of typical predicted and measured profiles of channels machined in PMMA. Predictions were obtained using the same procedure as described in Section 4.2.1 for glass except that the ductile surface evolution equation, Eq. (3.13), was solved, rather than the brittle. The values $\mathrm{n}_{1}, \mathrm{n}_{2}$, and $H v$ in Eq. (3.5) were taken as 1.27, 15.5, and $0.25 \mathrm{GPa}$, respectively, based on the data of Ref. [37]. The predictions were very good (max depth difference <4\%) up to an aspect ratio of 0.5 and even at higher aspect ratio of 2 (max depth difference $<8 \%$ ). The reason for the better agreement between model and experiments with PMMA 
than glass is the tendency of the PMMA channel sidewalls to become vertical very quickly since the maximum erosion occurs at shallow incident angles for such ductile materials. This rapidly developing steep sidewall prevents the second strike phenomena that occurs in glass channels.

It was found that, in general, the width of the frosted region in PMMA was no more than $11 \mu \mathrm{m}$ for the shadow mask and no more than $30 \mu \mathrm{m}$ for the maskless techniques, which was significantly less than glass. This can be attributed to the relatively low erosion rate of PMMA at normal incidence. Figure 3-18 shows a typical W shaped channel machined in PMMA.

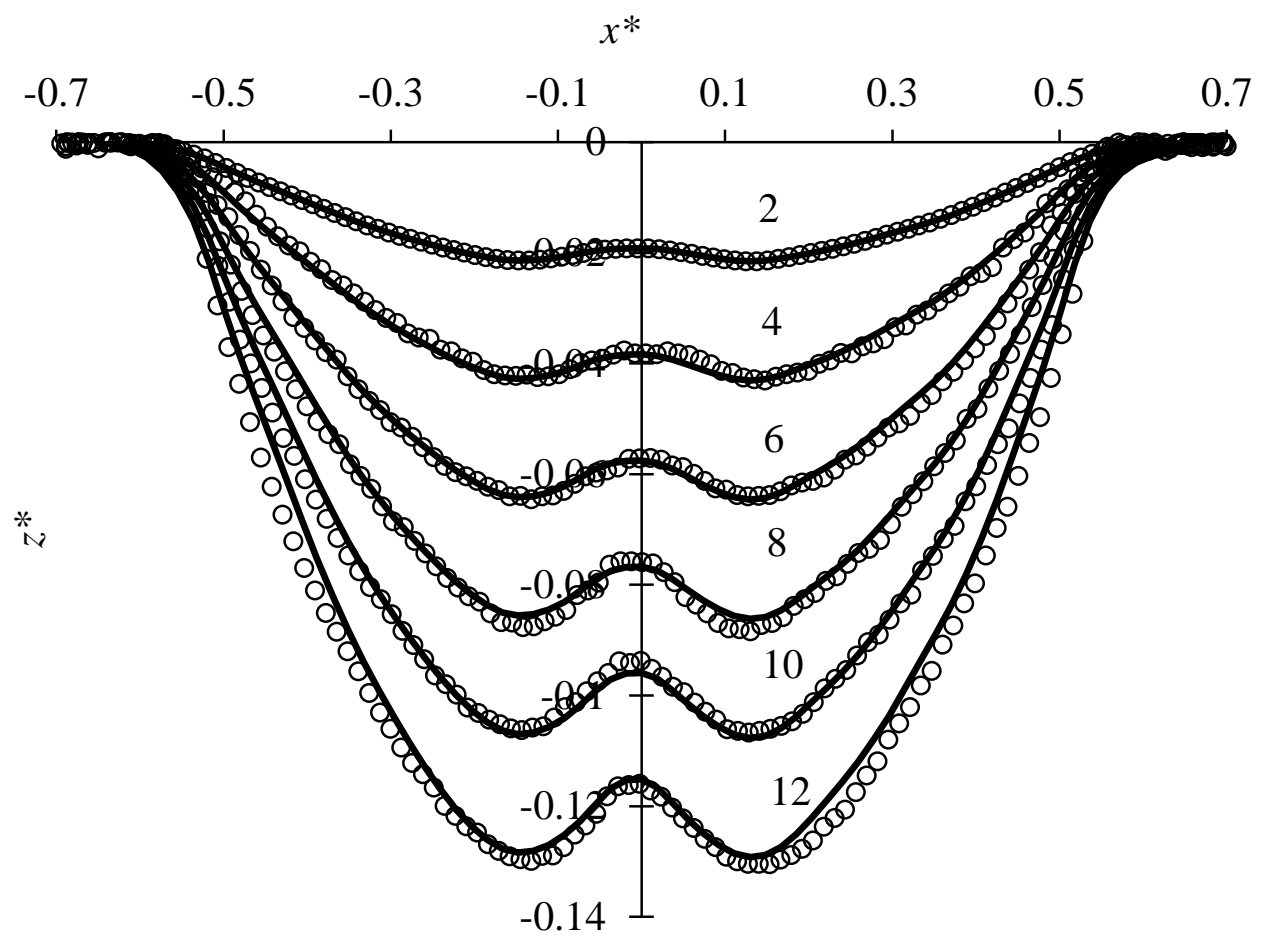

(a) 


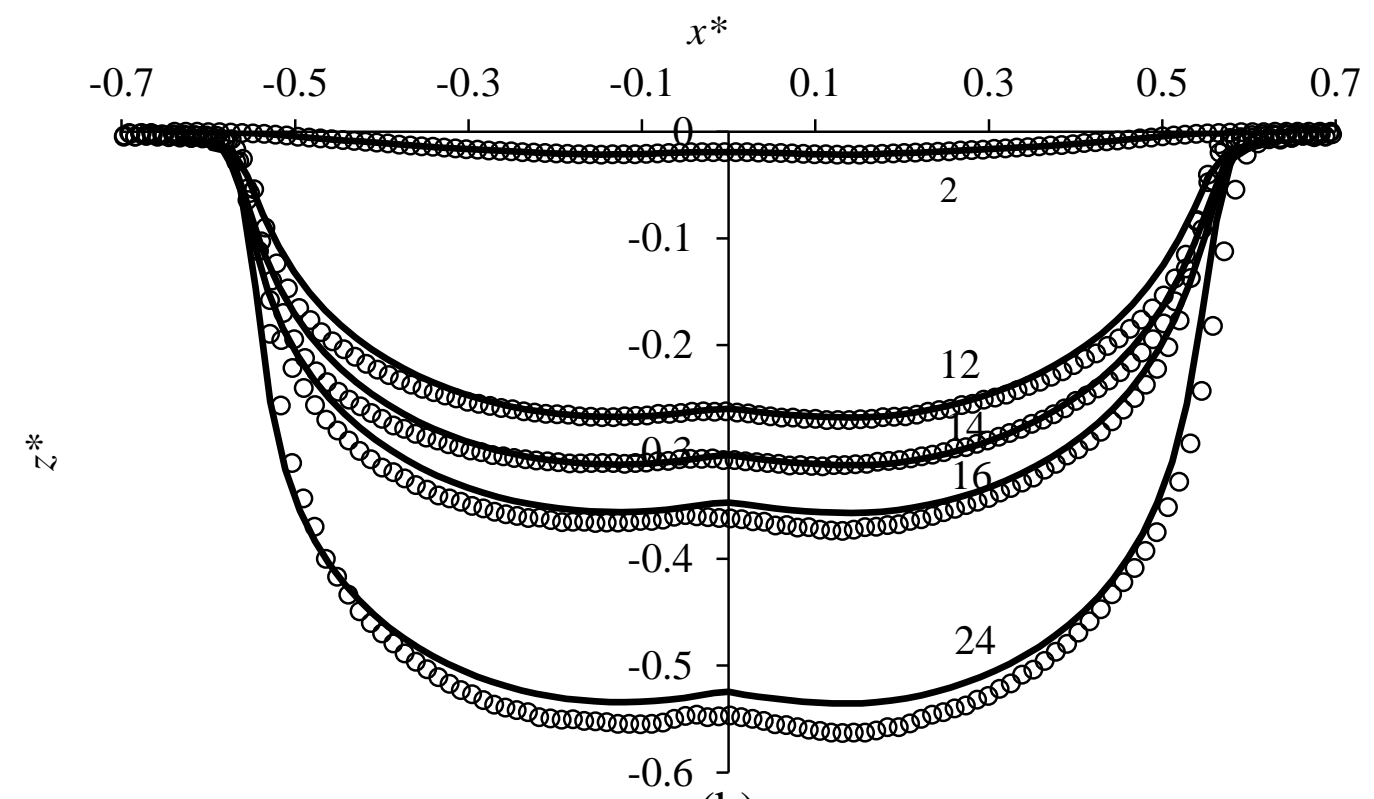

(b)

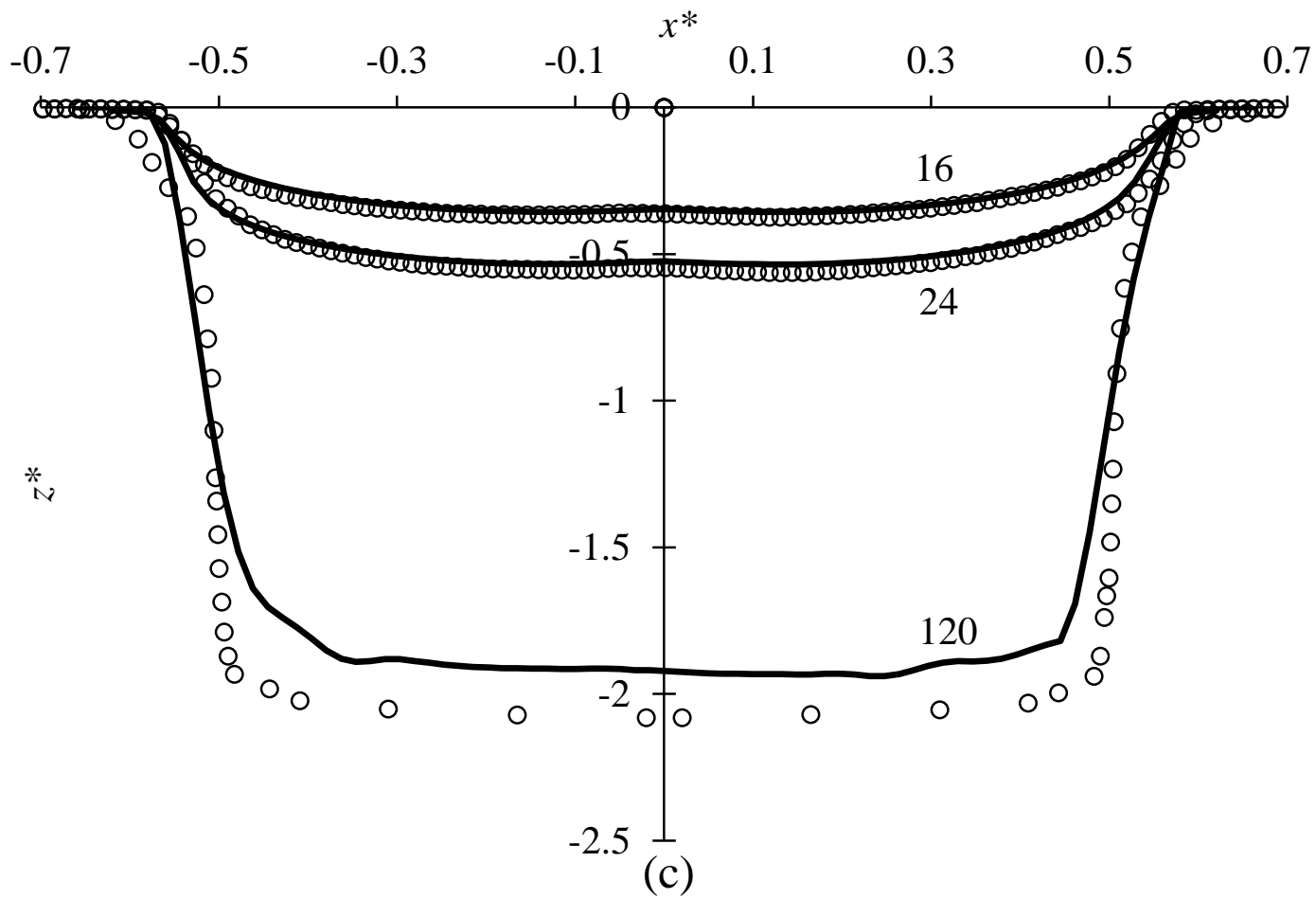

Figure 3-17 Comparison of predicted (symbols) and measured (lines) cross-sectional profiles of channels machined at amplitude of $320 \mu \mathrm{m}, v_{s}=1 \mathrm{~mm} / \mathrm{s}$ and $\omega=15 \mathrm{~Hz}$ in PMMA using maskless technique with various number of passes from experiments (symbols) and probability model (lines). The depth and width are normalized by $2 A+W_{f}=1081 \mu \mathrm{m}$. Number of passes is shown on each line. (a) shallow channels; (b) deeper channels; (c) very deep channels. 


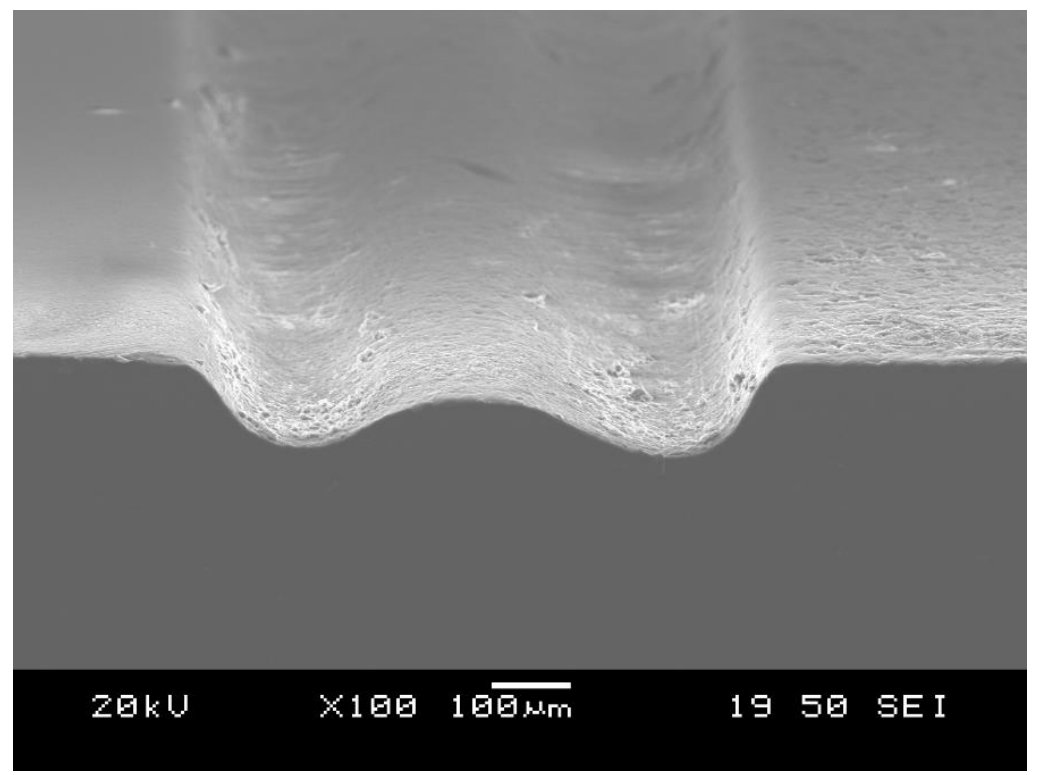

Figure 3-18 A typical W shaped channel machined in PMMA at amplitude of $250 \mu \mathrm{m}, v_{s}=1$ $\mathrm{mm} / \mathrm{s}$ and $\omega=15 \mathrm{~Hz}$ in PMMA using maskless technique.

The shadow mask can be used to make very narrow features by decreasing the mask opening. For example, Figure 3-19 shows a $400 \mu \mathrm{m}$ wide $\mathrm{W}$-shaped micro-channel made by using a mask opening of $150 \mu \mathrm{m}$.

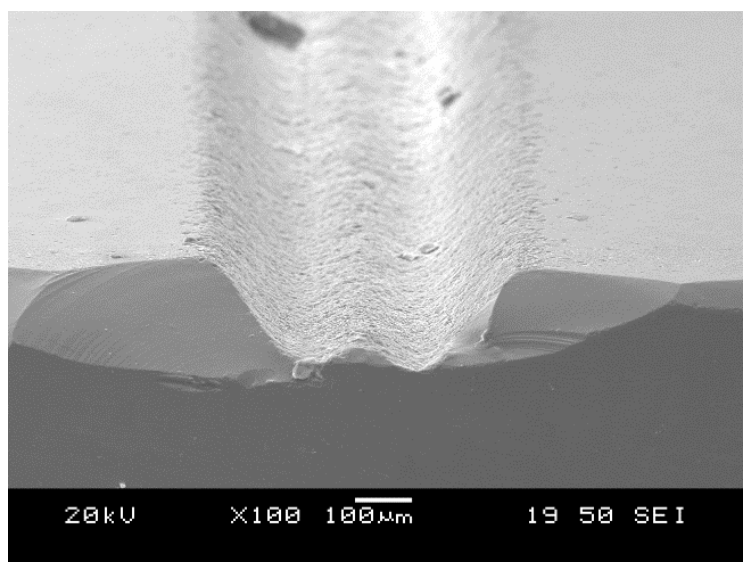

Figure 3-19 An example of small W shaped channel machined using shadow mask in PMMA. 


\subsubsection{Trapezoidal and U-shaped channels}

The $\mathrm{W}$ shaped channels machined using the techniques of Section 3.3 can be further machined to create trapezoidal (glass) or U-shaped (PMMA) channels by performing additional 'flattening' straight passes without oscillating along the centerline. To predict the proper input conditions for the additional centerline passes, an optimization routine was written in MATLAB and linked to the PDE solver in Mathcad.

The MATLAB code took as input the profile of a W-shaped channel machined using target oscillation, and the erosive efficacy measured from a 'flattening' shallow profile machined without oscillation at the same scan speed. The initial condition for the PDE was considered to be the Wshaped profile, and the objective was to determine the correct scan speed to erode the central peak sufficiently to make the middle area flat. The $\mathrm{W}$-shaped profile, the erosive efficacy for the flattening straight pass, and a trial scan speed for the flattening straight passes was sent from MATLAB to the Mathcad PDE solver. The surface evolution resulting from the flattening passes was performed and the predicted flattened profile was passed back to MATLAB, where it was compared with the desired flat profile. The average depth of the two deeper sections of the Wshaped channels was calculated and compared to the depth of the central peak. The scan speed of the non-oscillating pass required to remove the central peak was then predicted based on this depth difference, and passed back to the PDE solver in Mathcad to predict the flattened profile. This procedure repeated until the difference in depth was less than 5\%, and the appropriate scan speed for flattening was determined.

Figure 3-20 and Figure 3-21 (a) show examples of resulting trapezoidal channel machined using this technique. The agreement between predicted and measured profiles in Figure 3-20 is good, with a maximum error of 7\%. Figure 3-21 (b) shows that this technique gives rise to a more U-shaped channel in PMMA, owing to the more curved sidewalls of the original W-shape (Figure 3-18), compared to the more straight ones of glass. 


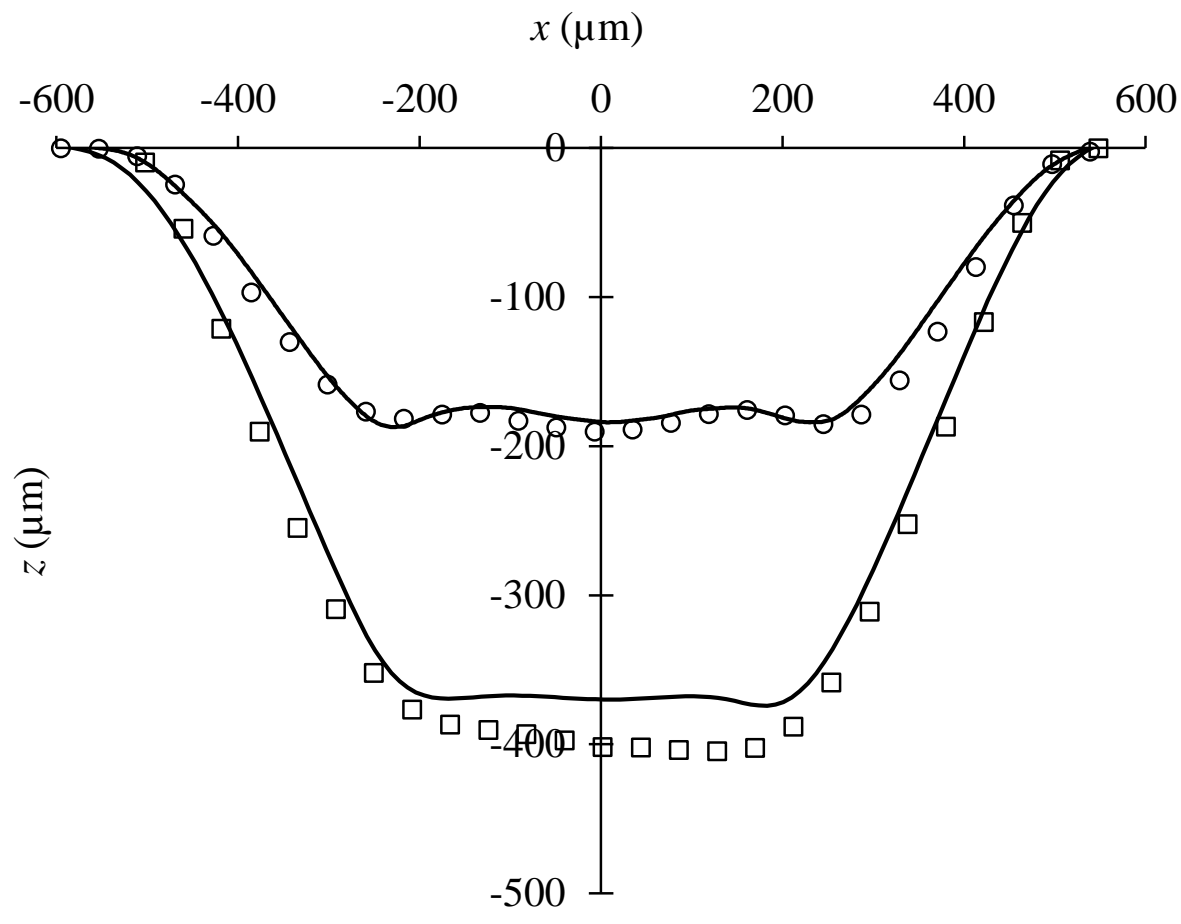

Figure 3-20 Predicted (lines) and measured (symbols) typical trapezoidal channel cross sections machined in glass by using oscillation method followed by additional passes at the center.

Oscillation was at $A=300 \mu \mathrm{m}$ and $\omega=15 \mathrm{~Hz}$, with one (circles) and two (squares) passes at $v_{s}=1$ $\mathrm{mm} / \mathrm{s}$, followed by 1 (circles) and 2 extra non-oscillating passes (squares), in the center with $v_{s}=$ $3.5 \mathrm{~mm} / \mathrm{s}$.

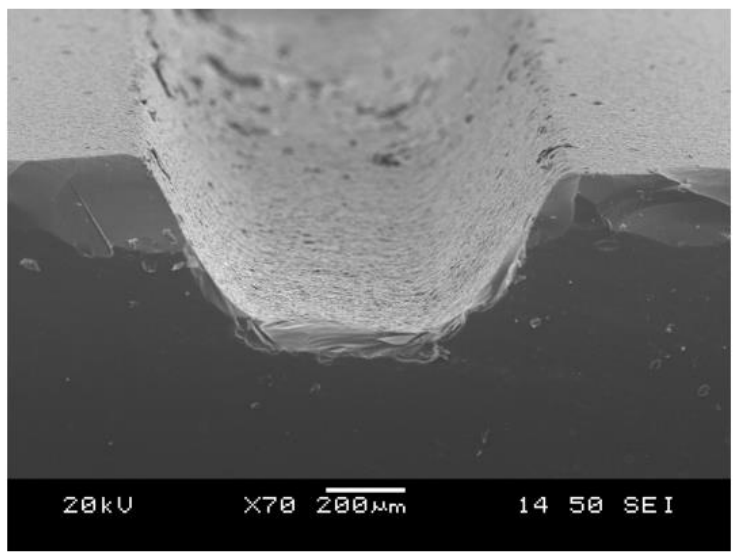

(a)

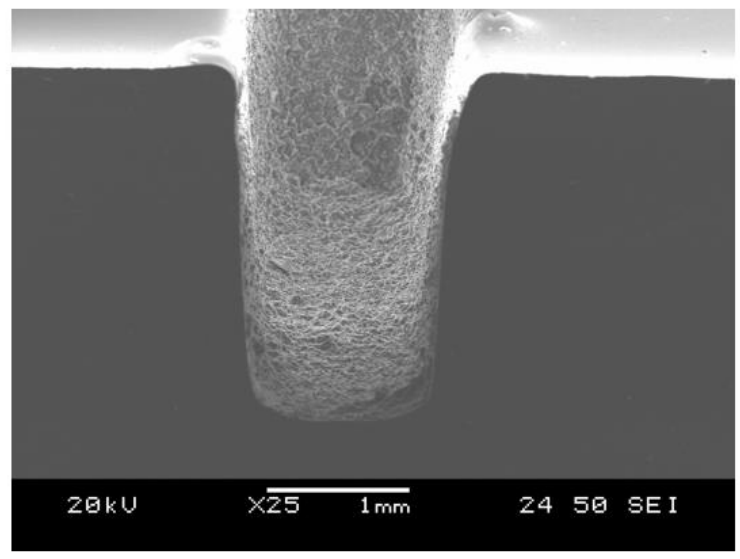

(b)

Figure 3-21 SEM images of trapezoidal and U shaped channels machined in (a) glass and (b) PMMA. 
This method of using additional passes to the middle of a W-shaped channel can also be used to machine other channel shapes that may find application in MEMS or micro-fluidics. For example, Figure 3-22 shows a triple via micro-channel that resulted from first machining a Wshaped channel, followed by three middle non-oscillating passes.

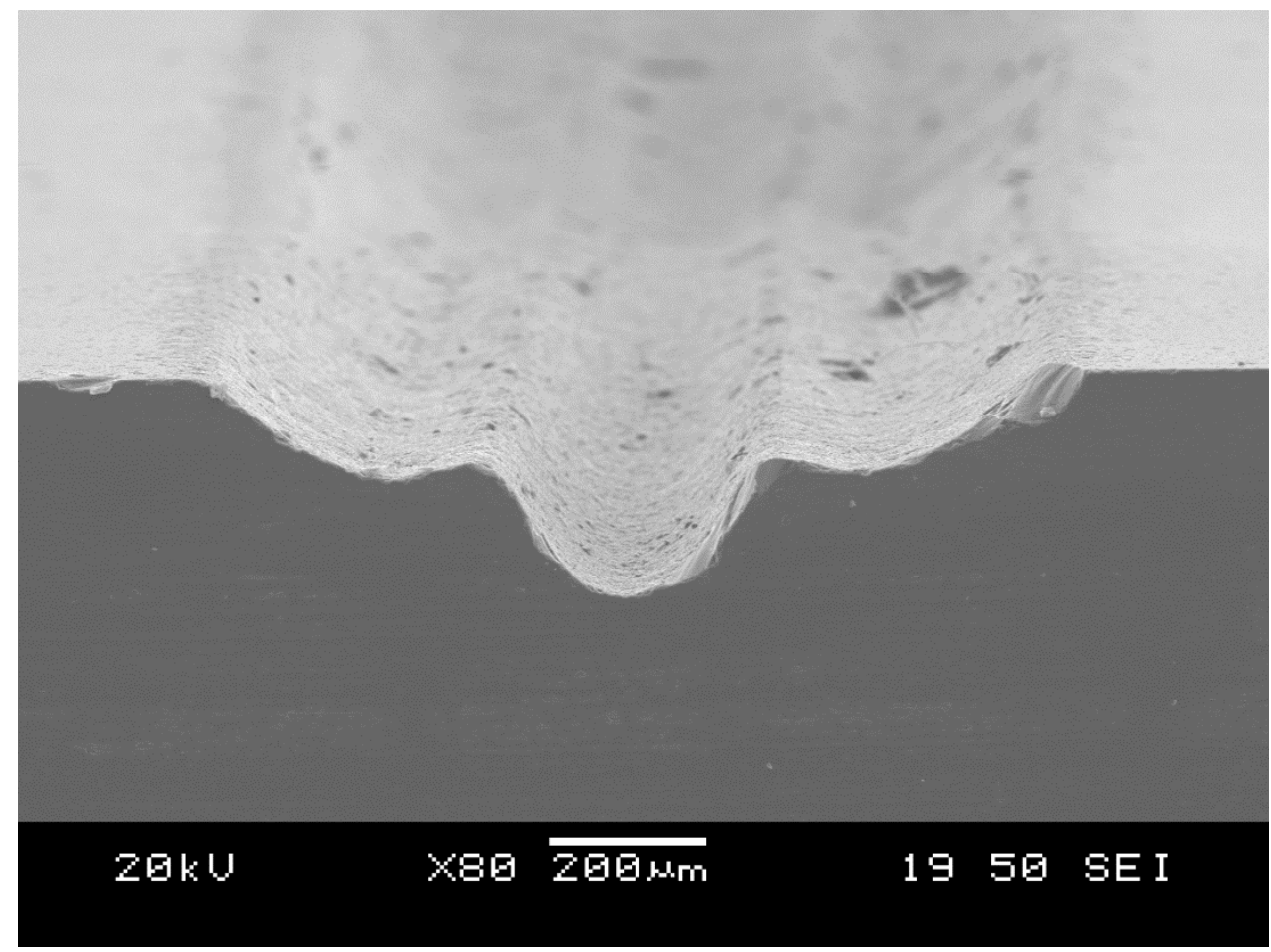

Figure 3-22 A triple via channel machined in glass by two oscillating passes $(\mathrm{A}=300 \mu \mathrm{m}, \omega=15$ $\mathrm{Hz}$ and $v_{s}=1 \mathrm{~mm} / \mathrm{s}$ ) followed by three middle non-oscillating passes with scan speed of $3.5 \mathrm{~mm} / \mathrm{s}$.

\subsection{Summary}

A novel AJM oscillating target methodology to sculpt a variety of U, W, V and trapezoidal cross-sectional micro-channel shapes into brittle (glass) and ductile (PMMA) targets was presented and modeled. Two techniques of obtaining a relatively small erosive efficacy footprint (maskless and shadow mask) were used with a sinusoidal target oscillation to create $\mathrm{W}$ and $\mathrm{V}$-shaped cross sections. The W-shaped profiles could be further processed to obtain trapezoidal (glass), U-shaped (PMMA), and other novel cross sections. The conclusions can be summarized as follows:

(i) Experiments revealed that tungsten carbide was the best material to use for the shadow-mask setup. 
(ii) Above certain threshold, the lateral oscillation frequency had no effect on the channel shape. W-shaped channels in PMMA were steeper and more curved than in glass.

(iii) Increases in longitudinal scan speed decreased the depth of the channels.

(iv) Low oscillation amplitudes resulted in V-shaped channels, which with increasing amplitude, switched to $\mathrm{W}$-shapes. For the same depth, a higher amplitude also led to a wider and taller central peak in the $\mathrm{W}$.

(v) The size of the erosive efficacy footprint produced by the nozzle, controlled using an adjustable shadow mask, strongly affected the shape and size of produced Wshapes. A larger footprint led to deeper and wider channels.

(vi) The $\mathrm{W}$-shaped profiles could be transformed into trapezoidal or U-shaped ones by performing additional passes along their centers, using an appropriate scan speed.

(vii) The predictions of the presented models agreed well with measured $\mathrm{W}, \mathrm{V}, \mathrm{U}$, and trapezoidal profiles. Typically, the maximum error was less than $7 \%$.

In Chapters 4 and 5, an inverse methodology will be presented that allows prediction of the required nozzle movement velocity to machine a variety of desired shaped micro-features, including $\mathrm{W}$-shapes. It will also be shown in Chapter 6 how a wider variety of $\mathrm{W}$-shapes than those presented in this chapter can be machined using a rotating mask apparatus. 


\section{Chapter 4 Inverse methods to gradient etch three-dimensional features with prescribed topographies using abrasive jet micro-machining: Modelling}

This chapter is based on the following published paper:

M. R. Sookhak Lari, and M. Papini. "Inverse methods to gradient etch three-dimensional features with prescribed topographies using abrasive jet micro-machining: Part I-Modeling." Precision Engineering, vol. 45, pp. 272-284, 2016.

\subsection{Introduction}

There is increasing interest in the micro-fabrication of devices having feature topographies different to those that result from traditional isotropic wet etching. This chapter aims to provide a relatively simple framework for determining the process parameters required to micro-machine features with specified desired cross-sectional shapes, textures, and/or with desired profiles of continuously varying depth, using abrasive jet micro-machining (AJM). Such a framework can find use in a variety of applications, but perhaps it is most important in micro-fluidics, where the channel cross-sectional shape can be a significant factor in the flow and heat transfer characteristics, and the probability of settling of micro-fluidic particles [2]. For example, Guan et al. [67] and Sharma et al. [68] have investigated the use of rectangular and trapezoidal microchannels, and Akbari et al. [69], Bahrami et al. [70] and Duan et al. [71] developed methodologies to analyze the flow in micro-channels of arbitrary cross-sectional shape. The waviness of a microchannel along its length can also affect the characteristics of fluid flow in micro-fluidic devices, and can offer advantages in controlling thermal and pressure distribution and dissipation. For example, changing the depth of a micro-channel gradually along it length or adding texture changes the surface area that the fluid is in contact with, and consequently the heat transfer [2]. This affects the fluid temperature, and thus its viscosity, possibly leading to desirable changes in the flow. Croce et al. [72] followed a numerical approach and presented the dependence of surface characteristics such as roughness on pressure drop and Nusselt number. Micro-channel texture has 
also been used to control the separation, mixing and velocity control of the flow in microfluidic devices [73].

Control of feature cross-sectional shape and texture requires technologies capable of anisotropic and gradient etching, but few such technologies currently exist that can be used in a simple and straightforward manner. Wilson et al. [54] combined micro-milling and soft lithography to make circular micro-fluidic channels in PDMS. Kumi et al. [55] fabricated different micro-fluidic channel cross-sections using high speed multiphoton absorption polymerization (MAP) in PDMS. Futai et al. [56] used backside diffused-light exposure to create bell shaped micro-channels, and made deformation-based micro-fluidic valves in PDMS. Anisotropically [70] and gradient-etched wedge-shaped channels [74] can be made using chemical etching on certain silicon materials, but the range of shapes that can be etched is limited by the orientation of the crystal planes. Unfortunately, all of these techniques are either relatively complex requiring multiple time consuming steps, cannot be used to create continuously curved features, or can be used to machine only a limited set of materials.

The directional and gradient etch capability of abrasive jet micro-machining (AJM) can be used to address many of the above identified shortcomings in the fabrication of three-dimensional (3D) and non-traditional shaped features [58]. AJM uses a jet of small particles passed through an erosion resistant mask to machine features into a variety of materials of interest for micro-fluidic, micro-electromechanical (MEMS) and opto-electronic devices. Typically, micro-channels have been made using AJM by scanning the jet at perpendicular incidence and a constant traverse (feed) speed over a mask opening on brittle materials such as glass, resulting in a constant-depth Vshaped profile [35]. However, channels etched in ductile materials, such as polymers, tend to result in a more U-shaped profile [37]. The differences in shape follow from differences in the local impact angle at which maximum erosion occurs for the particular target material [26]. Models that allow the prediction of the shape of constant-depth micro-channels machined in this manner (constant traverse velocity at perpendicular incidence) are well-established for both ductile and brittle materials $[1,3,31,36,37,42]$. Such models require a knowledge of the source erosive efficacy, a measure of the distribution of potential of the jet to erode a target surface. As demonstrated by Ghobeity et al. [39], this can most easily be determined by measuring a shallow channel or hole profile made using the source. 
A number of investigators have exploited the directional etch capability of AJM to fabricate three-dimensional (3D) components. For example, Yamahata et al. fabricated ball valve micropumps in glass [47] by employing AJM to make three dimensional micro-structures. Slikkerveer et al. [63] used two nozzles inclined at $60^{\circ}$ to the surface to create W-shaped micro-channels. Belloy et al. [75] further investigated oblique blasting for 3D micro-machining of brittle materials and examined the effect of secondary rebounding particles in mask underetching. Burzynski and Papini [32] used narrow band level set methods (LSM) to predict the shape of inclined channels machined at oblique incidence using AJM on glass and PMMA. AJM has also made it possible to machine a variety of suspended structures such as accelerometers in glass, millimeter size cantilever beams, complex micro-structures and monolithic micro-structures $[44,58]$. With the exception of Burzynski and Papini [32], none of these processes were rigorously modelled, and in all cases, models for predicting the process parameters leading to a particular desired shape were not presented.

Investigators working with a variety of manufacturing technologies have used partially overlapping adjacent offset straight machining passes, each at a constant traverse velocity, to create relatively wide features such as pockets, and provided superposition models to predict the resulting profiles. For example, using AJM, Ghobeity et al. [76] used such a procedure to create large (>6 $\mathrm{mm}$ ) planar pockets at various depths. Tamannaee et al. [77] recently used a similar technique for creating pockets and curved edges in a talc-filled polymer using an abrasive slurry jet (ASJ) machining technique. Kong et al. [78,79] developed a mathematical model to infer with good precision the 'footprint' (analogous to the AJM erosive efficacy) of a high-pressure abrasive waterjet (AWJ) for different machining paths, traverse velocities, and nozzle inclination angles, and used it to machine flat pockets using adjacent offset passes. They also showed that such a methodology could, in principle, also be used to predict the depth of a slot machined at variable velocity, although they were not able to further investigate this due to the process variations inherent to AWJ milling that resulted in unpredictable fluctuations in the etched profile. The use of overlapping adjacent machining passes to sculpt a surface is time-consuming, and not practical when features with sizes on the same order as the source footprint are required. The present chapter will present techniques to use AJM to fabricate very narrow ( 400 $\mu \mathrm{m}$ wide) non-flat features of a desired shape, by using traverse velocities that change instantaneously. 
Solution of the inverse problem to determine the source movement required to create a desired shape has not been considered thus far in AJM, and relatively few studies have been performed for other related processes. Booij [80] performed interesting exploratory work regarding the effect of continually changing radial nozzle velocity in the shaping and polishing of spinning disks using a fluid (water-abrasive slurry) jet polishing apparatus. Using a computer routine, they showed that by assuming a point source, they could predict reasonably well the radial velocity required to produce spherical and other simple shapes. Fang et al. [81] noting that numerical deconvolution often fails to converge, developed a discrete convolution algorithm to compute the dwell function in fluid jet polishing. Vasile et al. [82] presented a model to predict the dwell time for the ion-beam milling of different cavity shapes, using a Gaussian foot print based on calculation of the dwell time needed to machine each segment of the cross section. They also considered the overlapping effect of adjacent segments. Redeposition of removed material elsewhere on the target became important at high aspect ratios, and affected the accuracy of the results. Most recently, Bilbao Guillerna et al. [83] performed a frequency domain analysis to evaluate the feasibility of the abrasive waterjet machining of features with high frequency components. They solved the linear inverse problem by using a calibrated etching rate and a given target depth profile to calculate the required dwell time profile.

In summary, almost all previous research on AJM has focused on either (i) the use of multiple overlapping adjacent shallow passes, each with a constant traverse velocity, to create relatively wide features or (ii) the prediction of the shape of constant-depth micro-channels, given multiple traverses at a constant traverse velocity of an erosive efficacy source. Although the inverse problem has been considered for other manufacturing technologies, the solutions often require relatively complex deconvolution algorithms that do not always converge, and it is not clear whether they can be implemented or are even appropriate for AJM. This chapter uses analytical inverse deconvolution methods that can be used to predict, given a desired initial (shallow) micro-feature topography, the instantaneous non-uniform source velocity required to produce it. This nonlinear inverse problem has not been previously considered for AJM, and its solution will be shown to allow the micro-fabrication of a wide variety of three-dimensional features such as micro-channels and pockets with specified cross-sectional and/or lengthwise topographies. 


\subsection{Modelling}

The 'erosive efficacy' characterizes the potential of a source to erode a surface, and is analogous to the 'footprint' used in fluid jet polishing by e.g., Booij et al. [84], and in abrasive waterjet machining by Kong et al. [78]. The erosive efficacy, $E_{s}$, for AJM processes can be expressed as [39],

$$
\begin{aligned}
& E_{s}(x)=D \varphi(x)[V(x)]^{k} \\
& =\frac{d z(x)}{d t} \quad\left(\text { for small } \frac{d z}{d x}\right)
\end{aligned}
$$

where $\varphi(x)$ and $V(x)$ are the particle mass flux and velocity distributions, respectively, and $D$ and $k$ are experimentally determined erosion rate constants that depend, in general, on the particle and target characteristics. As shown previously [39], for a jet of particles incident in the $z$ direction perpendicular to a flat surface in the $x-y$ plane, it gives the initial rate $\mathrm{d} z / \mathrm{d} t$ at which the flat target surface will erode. It can therefore be easily approximated by measuring the $z$ profile of a shallow machined feature, and has been used together with appropriate two-dimensional surface evolution models to successfully predict the evolving time dependent shape ( $z$ profile) for constant-depth micro-channels machined at constant source traverse velocities in a variety of materials [37,39] . Such channels are typically either V-shaped (brittle targets) or U-shaped (ductile targets).

In order for more complex topographies to be made, the source must be appropriately accelerated and decelerated in either the $x$ (changing the cross sectional shape) or $y$ (changing the depth along the channel length) directions, or both. The following sections describe the methodology to determine the required instantaneous source velocity to obtain a specified topography for both of these cases.

\subsubsection{Total first pass erosive efficacy delivered by a moving source}

Figure 4-1 shows a source moving in a straight line above a surface at some traverse velocity, $v_{s}\left(s_{0}\right)$ such that its center is located at a point $S=s_{0}$ at time $t$. 


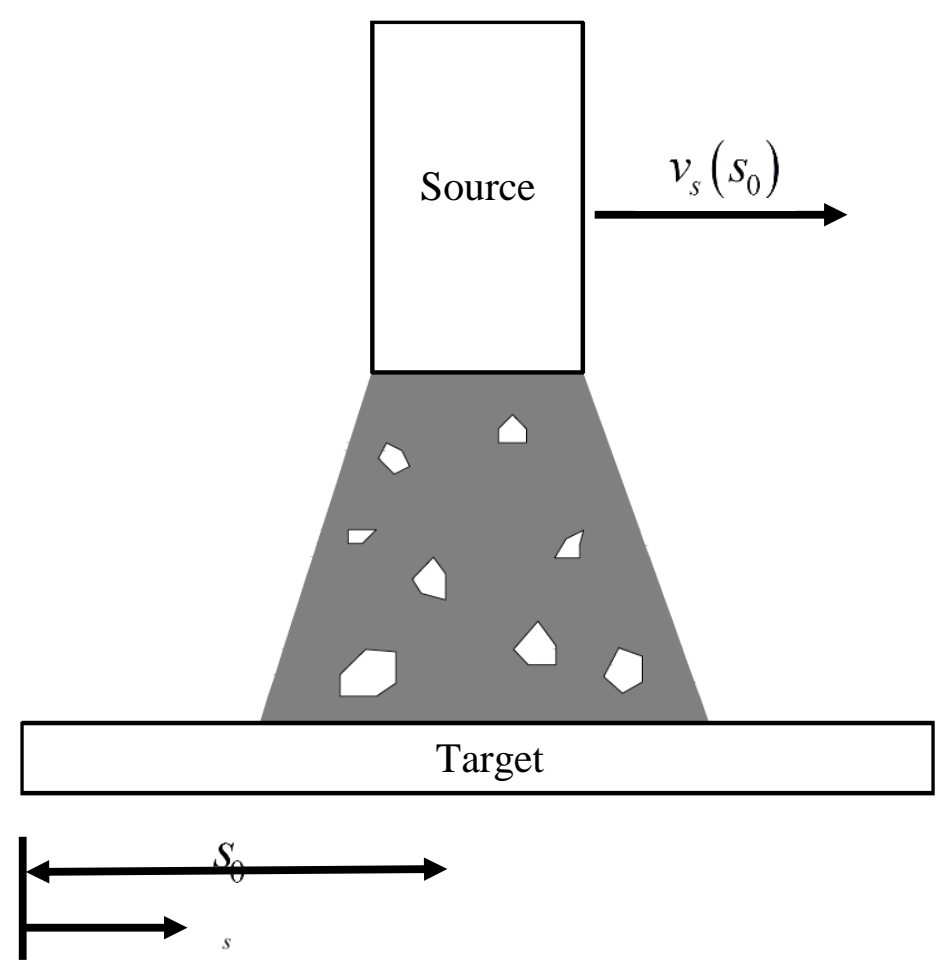

Figure 4-1 Schematic showing moving AJM source whose centerline is instantaneously located at $s=s_{0}$.

As discussed in Section 3.3.2, the proportion, $d t$, of the total traverse time $T=t_{f}-t_{i}$ which the source center spends between $S=s_{0}$ and $s_{0}+d s_{0}$, or equivalently the probability, $P\left(s_{0}\right)$ d $s_{0}$ of finding the source center between these locations is

$$
\frac{d t}{T}=P\left(s_{0}\right) d s_{0}
$$

Since $v_{s}\left(s_{0}\right)=d s_{0} / d t$ this gives the result

$$
P\left(s_{0}\right)=\frac{1}{T v_{s}\left(s_{0}\right)}
$$


Therefore, in moving from $s_{i}$ to $s_{f}$, the total erosive efficacy delivered by the moving source is

$$
\mathrm{E}_{T}(s)=\int_{s_{i}}^{s_{f}} E_{s}\left(s-s_{0}\right) P\left(s_{0}\right) d s_{0}=\int_{s_{i}}^{s_{f}} \frac{E_{s}\left(s-s_{0}\right)}{T v_{s}\left(s_{0}\right)} d s_{0}
$$

where $E_{s}(s)$ is the spatial distribution of the source erosive efficacy, which as discussed previously, can be determined from a shallow profile eroded using a stationary source, or as will be shown in Section 4.2.4.3.1, moving at a constant $v_{s}$.

\subsubsection{Source velocity required to obtain a desired initial topography}

The initial shallow shape of the desired topography resulting from moving the source can be related to the left hand side of Eq. (4.4). Assuming that the slope $d z / d s$ along the cutting front remains small during the first pass occurring over a time $T$, Eqs. (4.1) and (4.4) imply that the initial machined profile due to scanning the nozzle will be approximately

$$
E_{T}(s) T=z_{1}(s)=\int_{s_{i}}^{s_{f}} \frac{E_{s}\left(s-s_{0}\right)}{v_{s}\left(s_{0}\right)} d s_{0}
$$

If the source erosive efficacy $E_{s}$ is known, and if an initial particular topography is desired (so that $z_{1}(s)$ is specified), then the required velocity function can be determined by solving this Fredholm integral equation of the first kind with difference kernel $E_{s}$. Depending on the particular problem, it may be more appropriate or convenient to express the end limit of integration as variable, i.e. $s_{f}=s$, in which case a Volterra equation results, or one or both limits of integration as infinite, in which case a convolution or Wiener-Hopf equation results [85].

In the case of a variable limit (i.e., upper limit of Eq. (4.5) is $s$ ), then the resulting Volterra equation, in theory, can be solved using a Laplace transform technique [85]. However, since most kernels corresponding to realistic AJM source erosive efficacy distributions follow a Gaussian or more complex form, the inverse Laplace transform required of the solution is often difficult or impossible to obtain. Figure 4-2 shows two typical erosive efficacies for AJM sources, a Gaussian 
one obtained using the shadow mask apparatus of Chapter 2 and a more complex one obtained using a maskless nozzle at a very low standoff distance.

In the case where Eq. (4.5) has infinite limits, a convolution type integral equation results [85]. As mentioned in the introduction, while numerical deconvolution algorithms developed for other machining processes exist they are often complex, difficult to implement, and do not converge. Below it will be demonstrated that if the source erosive efficacy is Gaussian, then the theory of Gauss transforms [86,87] can be used obtain an analytical solution to any desired accuracy.

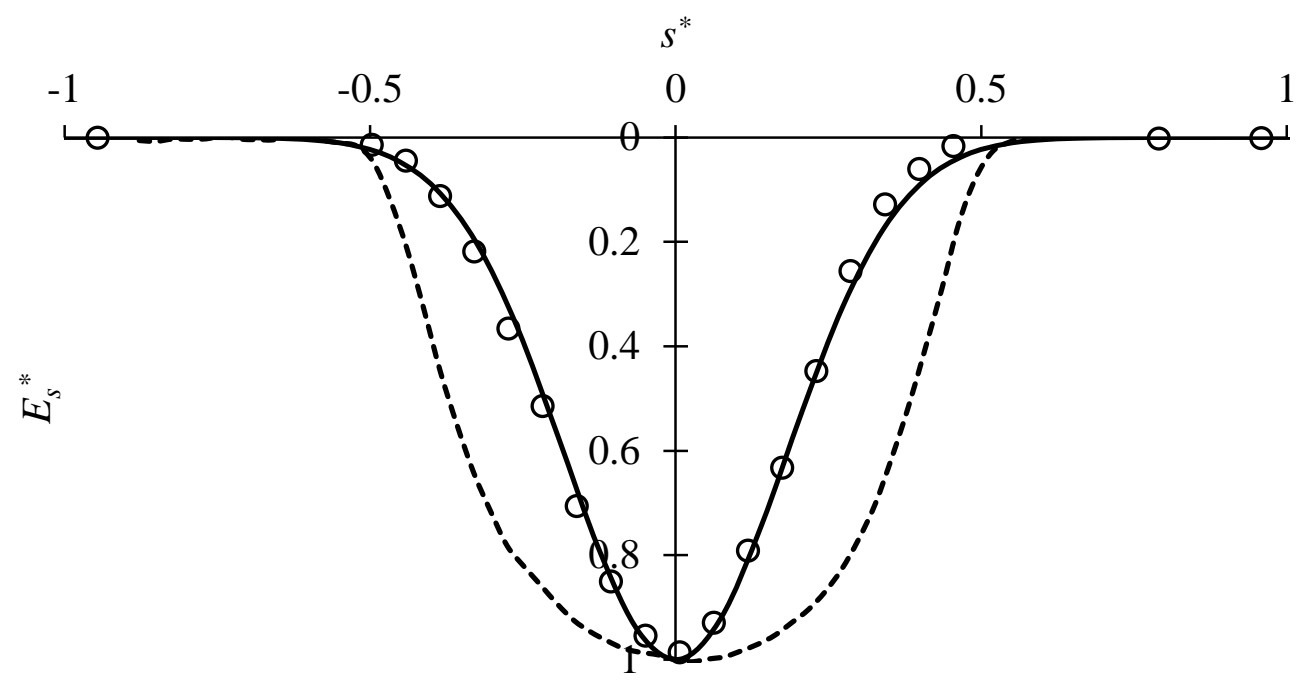

Figure 4-2 Non-dimensionalized erosive efficacy through a $490 \mu \mathrm{m}$ shadow mask opening (circles), and a maskless circular source of $460 \mu \mathrm{m}$ diameter at $100 \mu \mathrm{m}$ standoff (dashed line). The solid line shows the least squares best fit curve of Eq. (4.6) with $\beta=5.9$, assuming that the

length dimensions are normalized by the width of the footprint $(550 \mu \mathrm{m})$, and the erosive efficacy by its value at $s^{*}=0$.

\subsubsection{Analytical solution for Gaussian kernels}

The measured erosive efficacy for both shadow-masked nozzles, and unmasked nozzles at large distances [39], often have the following non-dimensional (starred quantities are dimensionless) form,

$$
E_{s}^{*}\left(s^{*}\right)=e^{-\beta^{2} s^{* 2}}
$$


where $\beta$ is a focus coefficient characterizing the footprint dimensions that can be obtained from a shallow profile eroded by the source moving in the $s$ direction, and $E_{s}^{*}=\frac{E_{s}}{E_{s}(0)}$. It is noted that the numerical value of $\beta$ depends on the factor that is used to non-dimensionalize $s$ in order to obtain $s^{*}$. An example for a shadow mask giving $\beta=5.9$ if the width of the source is the normalizing factor, is shown in Figure 4-2.

It is convenient to non-dimensionalize Eq. (4.5) using $z_{1}^{*}=\frac{z_{1}}{z_{1}^{\min }}, s^{*}=\frac{s}{z_{1}^{\min }}, v_{s}^{*}=\frac{v_{s}}{E_{s}(0)}$, $E_{T}^{*}=\frac{E_{T}}{E_{s}(0)}, T^{*}=\frac{T E_{s}(0)}{z_{1}^{\min }}$ and multiply by $\frac{\beta}{\sqrt{\pi}}$ to obtain

$$
\frac{\beta}{\sqrt{\pi}} z_{1}^{*}\left(s^{*}\right)=\frac{\beta}{\sqrt{\pi}} \int_{-\infty}^{\infty} \frac{e^{-\beta^{2}\left(s^{*}-s_{0}^{*}\right)^{2}}}{v_{s}^{*}\left(s_{0}^{*}\right)} d s_{0}^{*}
$$

The normalizing length $z_{1}^{\min }$, is the minimum depth of the desired feature machined as the nozzle moves during a total time $T$. The right hand side of Eq. (4.7) can be recognized as the Gauss transform, and an analytical solution for the velocity function which repeats indefinitely can be obtained in terms of the following series [85].

$$
v_{s}^{*}\left(s^{*}\right)=\frac{1}{\frac{\beta}{\sqrt{\pi}} \sum_{j=0}^{\infty} \frac{1}{\mathrm{j} !}\left(\frac{-1}{4 \beta^{2}}\right)^{j} \frac{d^{2 j} z_{1}^{*}}{d s^{* 2 j}}}
$$

Moreover, noting that any desired profile, $z_{1}^{*}\left(s^{*}\right)$, can be expressed to a desired accuracy as a Fourier series with that is periodic over an interval $s^{*}=[-P, P]$, and making use of the following higher derivative rules, 


$$
\frac{d^{n} \sin (s)}{d s^{n}}=\sin \left(s+\frac{n \pi}{2}\right) ; \quad \frac{d^{n} \cos (s)}{d s^{n}}=\cos \left(s+\frac{n \pi}{2}\right)
$$

leads to,

$$
v_{s}^{*}\left(s^{*}\right)=\frac{1}{\frac{\beta}{\sqrt{\pi}}\left[\frac{a_{0}}{2}+\sum_{j=0}^{\infty} \frac{1}{\mathrm{j} !}\left(\frac{-1}{4 \beta^{2}}\right)^{j} \sum_{n=1}^{\infty}\left[a_{n}\left(\frac{n \pi}{P}\right)^{2 j} \cos \left(\frac{n \pi s^{*}}{P}+\pi j\right)+b_{n}\left(\frac{n \pi}{P}\right)^{2 j} \sin \left(\frac{n \pi s^{*}}{P}+\pi j\right)\right]\right]}
$$

where the Fourier series coefficients

$$
\begin{gathered}
a_{0}=\frac{1}{P} \int_{-P}^{P} z_{1}^{*}\left(s^{*}\right) d s^{*} \\
a_{n}=\frac{1}{P} \int_{-P}^{P} z_{1}^{*}\left(s^{*}\right) \cos \left(\frac{n \pi s^{*}}{P}\right) d s^{*} \\
b_{n}=\frac{1}{P} \int_{-P}^{P} z_{1}^{*}\left(s^{*}\right) \sin \left(\frac{n \pi s^{*}}{P}\right) d s^{*}
\end{gathered}
$$

can be obtained using standard techniques, and it is assumed one wavelength of the desired feature is centered at $s^{*}=0$. It is noted that, although the solution in Eq. (4.8) is strictly valid for $z_{1}{ }^{*}$ that repeats indefinitely, it can be also be bound over an interval of interest, e.g. $s^{*}=\left[s_{i}{ }^{*}, s_{f}{ }^{*}\right]$. This solution will be demonstrated in Section 4.3, and verified experimentally in Chapter 5, to be effective for predicting the non-uniform linear velocity to obtain a desired repeating texture along the length of a channel and, with some approximations, the source oscillation velocity waveform necessary to sculpt a channel with a desired cross-sectional shape. 


\subsubsection{Point source}

Unfortunately, in some cases (e.g. the maskless dashed line in Figure 4-2), the source footprint (kernel of Eq. (4.5)) is non-Gaussian, and closed-form solutions do not exist. In these cases, an approximate solution can be obtained by assuming a point source $E_{s}\left(s-s_{0}\right)=K \delta\left(s-s_{0}\right)$ where $\delta$ is the Dirac delta function and $K$ a constant. The constant $K$ must have length ${ }^{2} /$ time units $^{2}$ to maintain dimensional compatibility with the definition of the erosion efficacy. If a channel of constant depth $z_{c}$ is made by scanning at a constant speed of $v_{c}$, then $K=z_{c} v_{c}$. Equation (4.5) can be put in non-dimensional form as,

$$
z_{1}^{*}\left(s^{*}\right)=\int_{s_{i}^{*}}^{s_{f}^{*}} \frac{\delta^{*}\left(s^{*}-s_{0}^{*}\right)}{v_{s}^{*}\left(s_{0}^{*}\right)} d s_{0}^{*}
$$

where $z_{1}^{*}=\frac{z_{1}}{z_{1}^{\min }}, s^{*}=\frac{s}{z_{1}^{\min }}, \delta^{*}=\delta z_{1}^{\min } v_{s}^{*}=\frac{v_{s} z_{1}^{\min }}{K}, E_{T}^{*}=\frac{E_{T} z_{1}^{\min }}{K}, T^{*}=\frac{T K}{\left(z_{1}^{\min }\right)^{2}}$ with $z_{1}^{\min }$ and $T$ defined as before. Equation (4.14) has the solution

$$
v_{s}^{*}\left(s^{*}\right)=\frac{H\left(s^{*}-s_{i}^{*}\right)-H\left(s^{*}-s_{f}^{*}\right)}{z_{1}^{*}\left(s^{*}\right)}
$$

where the Heaviside functions $H$ limit the bounds of the solution between $s_{i}{ }^{*}$ and $s_{f}{ }^{*}$.

Equation (4.15) implies that for a point source of erosive efficacy, the required velocity function in the position $(s)$ domain is directly proportional to the inverse of the desired profile. Conditions under which the point source approximation is effective will be discussed in Section 4.3.1.

\subsubsection{Predicting the topography of deeper profiles}

The methodologies presented in Sections 4.2.2.1 and 4.2.2.2 give the required instantaneous source velocity $v_{s}(s)$, to produce a particular desired shallow topography, i.e., a topography $z_{1}(s)$ with relatively shallow sidewalls resulting from a relatively rapid single pass. The 
total erosive efficacy resulting from this single pass, $E_{T}(s)$, can then be determined from Eq. (4.5) , and subsequently used in previously developed surface evolution equations to predict the evolution of the $z$ profile as the feature becomes deeper in response to multiple repeated identical passes. This is necessary because the erosion rate, in general, depends on the local particle impact angle, so that, e.g., the center of a relatively deep $U$ shaped channel etches at a significantly different rate than the sloped sidewalls where the particles strike at a more glancing impact [65].

The appropriate partial differential equations describing the surface evolution for brittle and ductile targets are Eqs. (3.2) and (3.4), respectively. Besides the erosive efficacy $E_{T}(s)$, they also require knowledge of the dependence of the erosion rate on incident angle and velocity, which can be found experimentally. Such surface evolution equations have been commonly used to predict the evolving two-dimensional cross sectional profile of uniform (along their length) depth channels. Section 4.2.4 discusses how they can be used to predict three-dimensional profiles for certain special cases in which the depth varies along the etched feature length.

\subsubsection{Gradient etching techniques}

Depending on the type of desired feature, the source may need to move in different directions, resulting in different methodologies to apply the solutions presented in Section 4.2.2 in order to obtain the required source velocity function.

\subsubsection{Channel with desired texture along its length}

If the aim is to create texture along the length of a micro-channel, e.g., a sloped, sinusoidal, etc., varying depth along the $y$ direction, then the nozzle moves at varying speed in the $y$ direction while remaining stationary in the $x$ direction (Figure 4-3). 


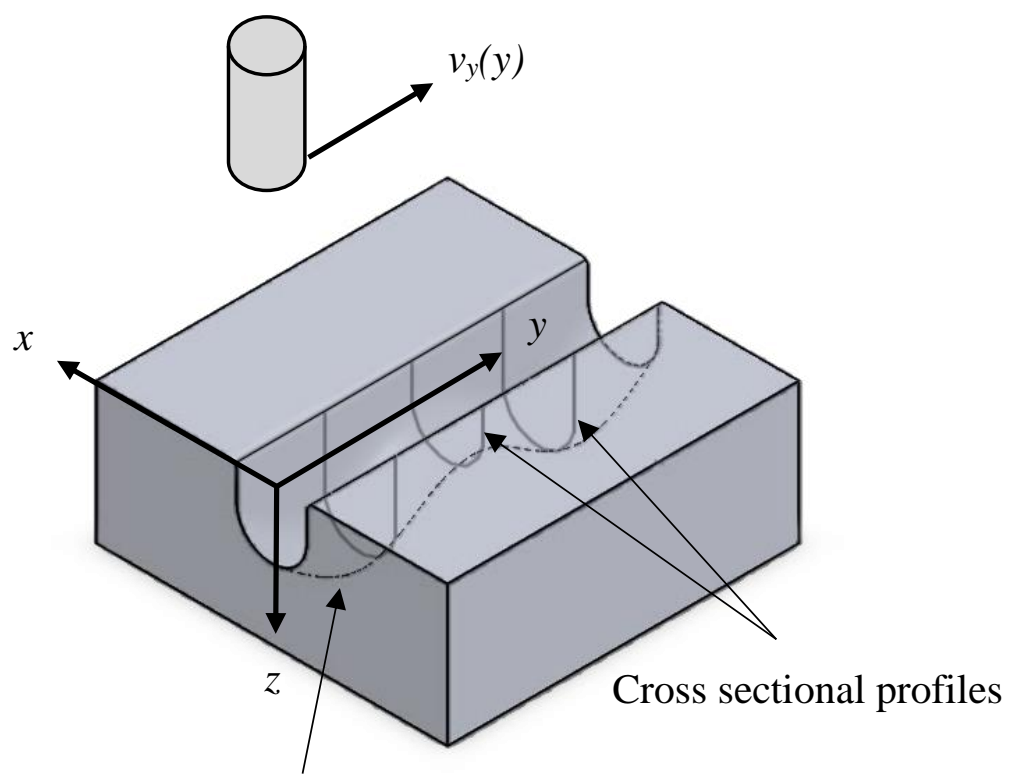

Centerline profile $z(x=0, y)$

Figure 4-3 Source moving in $y$-direction at velocity $v_{y}(y)$ to create a channel with a varying depth.

In this case, $s$ is replaced by $y$ in Eq. (4.5), thus relating the desired channel centerline profile (along $y$, at $x=0$, Figure 4-3) to that of the source. The methods of Section 4.2.2.1 (finite Gaussian source for small features) or 4.2.2.2 (point source for large features) can then be used to obtain the required velocity to obtain a desired shallow first pass centerline profile, $z_{1}{ }^{*}\left(y^{*}\right)$, and Section 4.2.3 to determine how it evolves over multiple passes. Visualization of the predicted full three-dimensional (3D) profile, however, requires a method to determine the cross-sectional profile at every $y$ location (Figure 4-3). To do this, the desired channel centerline $z^{*}\left(x^{*}=0, y^{*}\right)$ was used as the final depth required to be machined at any $y^{*}$. The symmetry of the channel in the $x$-direction produced by a nozzle at perpendicular incidence implies that the slope $\mathrm{d} z^{*} / \mathrm{d} x^{*}$ on the center line of the channel is zero at any depth. Therefore, Eq. (4.1) implies a linear relationship between time and centerline depth:

$$
t^{*}\left(y^{*}\right)=\frac{z^{*}\left(y^{*}\right)}{E_{s}^{*}(0)}
$$


in which $E_{s}^{*}(0)$ is the erosive efficacy at the center of the channel $\left(x^{*}=0\right)$. Therefore, the channel cross-sectional profiles $z^{*}\left(x^{*}\right)$ at each $y^{*}$ location can be obtained by solving the surface evolution equations described in Section 4.2.3 up to a time $t^{*}\left(y^{*}\right)$.

In solving the surface evolution equations, the partial differential equation (PDE) solver, which uses the method of lines, in Mathcad 15 (PTC Inc. Needham, MA, USA) was used with the initial condition $z^{*}\left(x^{*}, t^{*}=0\right)=0$. By performing grid size analysis on the convergence of the results, it was found that $\Delta t=100$ and $\Delta x=500$, time and space steps, respectively were sufficient to maintain the accuracy of the numerical solution for any given two-dimensional cross-sectional profile. The cross-sectional profiles were obtained at $\Delta y=5 \mu \mathrm{m}$ intervals and connected using a specially written MATLAB code that generated a matrix of data points at different $x, y$ and $z$ locations, to ultimately produce the visualization of the three-dimensional channel profiles $z^{*}\left(x^{*}, y^{*}\right)$. Figure $4-4 \mathrm{c}$ gives an example of such a generated profile, and the methodology will be extended for pocket textures in Section 4.2.4.2.

\subsubsection{Pockets with texture in two directions}

Pockets with texture in both the $x$ and $y$ directions (e.g, Figure 4-4a) can be made by machining straight channels, each with its own $y$-direction texture, side by side in the $x$-direction. The following steps are required in order to predict the required velocity function to achieve these more complex textures:

(i)The desired texture pattern is discretized into multiple strips of the same width along one direction, typically the one with the longer wavelength, e.g., the $y$-direction in Figure 4-4. The size of these strips, $w_{s}$, will affect the resolution of the machined profile. In general, a degree of overlap of adjacent strips, i.e., use of strip sizes narrower than the source footprint width, is desirable. For example, Figure 4-4b shows three and their corresponding centerline profiles.

(ii) The shallow centerline profiles of each strip from Step 1 are extracted and used to predict the set of required velocity functions required to yield them using the same procedure as in Section 4.2.4.1. The resulting velocity functions will yield the predicted erosive efficacies for each strip (Eq. (4.5)). The profiles of each of the 3D strips (Figure 4-4c) can be then generated as discussed in Section 4.2.4.1. 
(iii) A 3D visualization of the final texture can be obtained by taking into account the partial overlap of strips (Figure 4-5). This overlap means the erosive efficacies must be summed, similar to what was suggested by Tamannaee et al. [77] for the abrasive slurry jet machining of shallow flat pockets and curved edges into polymers. However, instead of flat bottoms, the presently considered pockets had textures that changed along each strip, and also in the direction normal to the strip. Therefore, the profiles needed to be superimposed in $3 \mathrm{D}$ by marching along the $y$ direction in $5 \mu \mathrm{m}$ increments, summing the $x$-direction erosive efficacies of each overlapping adjacent pass at different $y$-locations. The resulting net 3D erosive efficacy could then be obtained by connecting the $x$-direction erosive efficacies obtained at each $y$, using the same MATLAB code as that used to create 3D profiles in Section 4.2.4.1. Figure 4-4d shows an example of the result for three adjacent strips.

Since step (ii) predicts the velocity required to produce the centerline of each strip in one direction only, it cannot directly predict the effect of overlapping adjacent strips. The resolution of final pattern is thus related to the relative magnitudes of the strip width $w_{s}$, the size of overlapped area $w_{o}$, and the width of the erosive efficacy $w_{e}$. As will be shown in Chapter 5 , it was found that the difference between predicted and measured texture was less than $7 \%$ when $w_{s}>0.75 w_{e}$ which ensured that the effect of summing the erosive efficacy of adjacent strips had a minimal effect on the centerline profiles of the adjacent strips. 


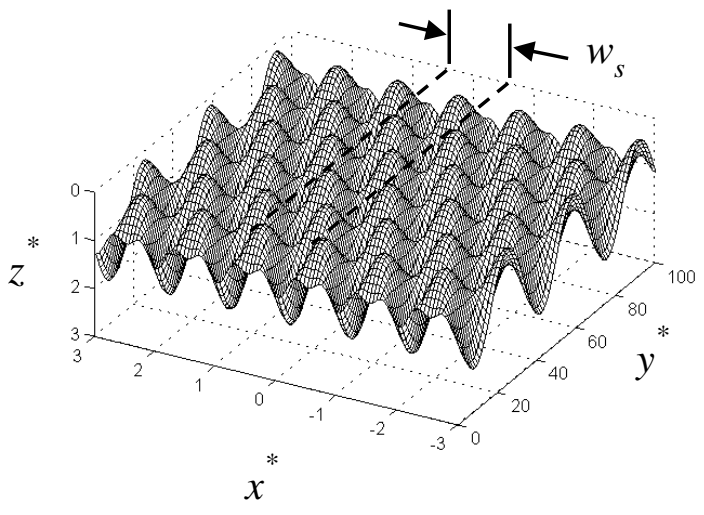

(a)

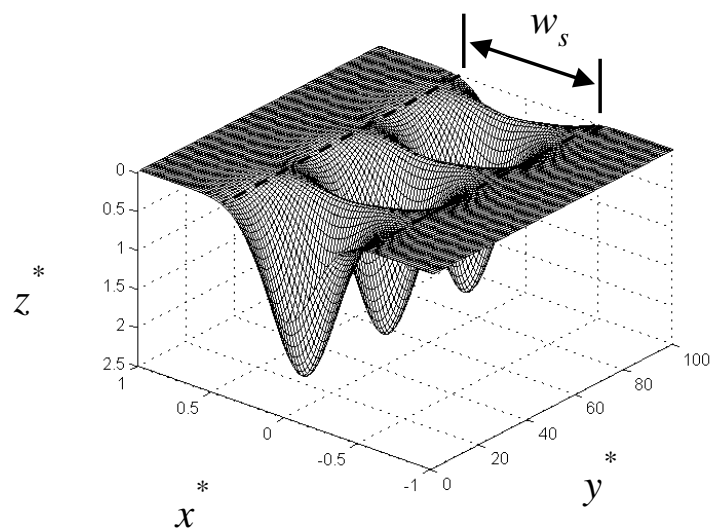

(c)

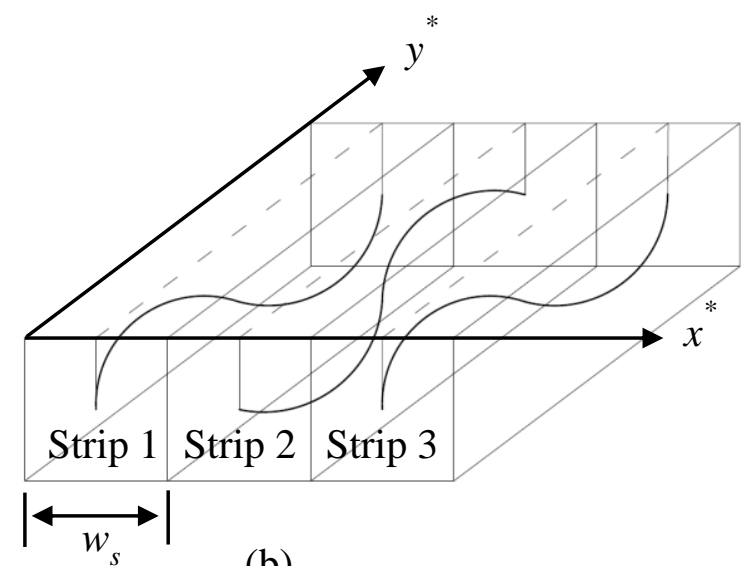

(b)

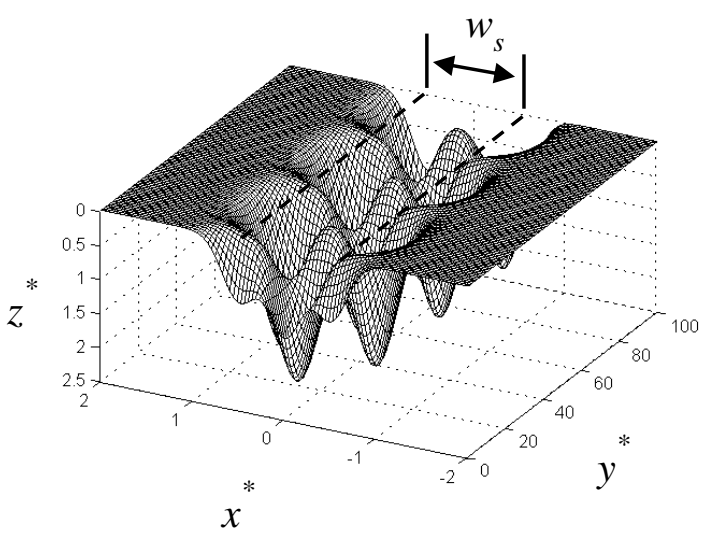

(d)

Figure 4-4 Prediction of source velocity and path to obtain desired pocket texture. (a) Desired double sinusoidal texture on a pocket having wavelength and amplitude of, respectively, 2 and 1, and 100/3 and 1, in the $x$ and $y$ directions, respectively. (b) Centerline profiles of three adjacent strips. (c) Predicted profile of one strip. (d) Resultant texture from three adjacent strips. 


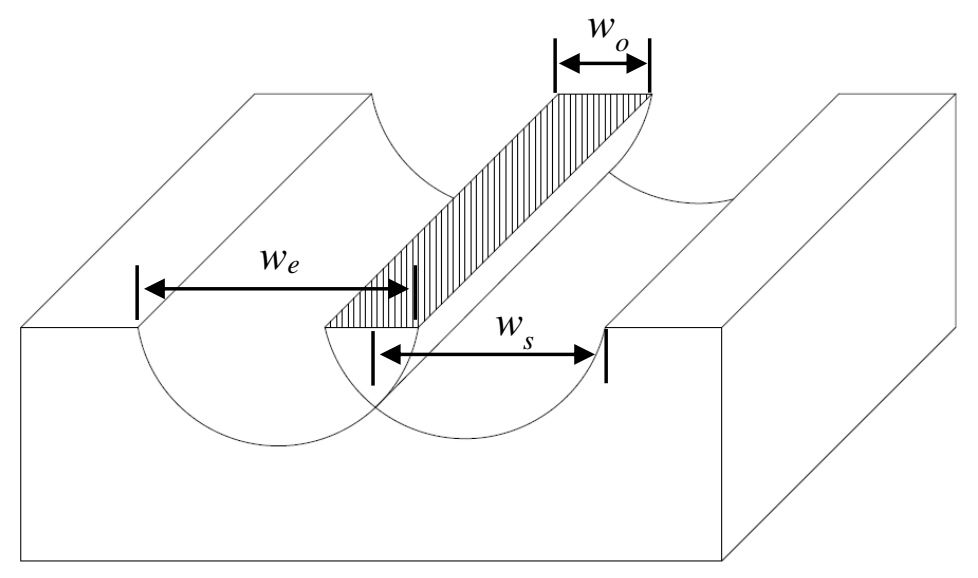

Figure 4-5 Schematic of two adjacent strips showing strip width $\left(w_{s}\right)$, width of erosive efficacy $\left(w_{e}\right)$ and width of overlapped area $\left(w_{o}\right)$.

To predict deeper pocket textures, the centerline of each strip can be used in the surface evolution equations described in Section 4.2.3. The 3D profile of each strip would be then formed using these deep centerlines and the final texture can be calculated using the same summation method as described above for shallow textures.

\subsubsection{Channel with prescribed cross-sectional shape - Gaussian oscillating source}

If the aim is to control the cross-sectional shape of a channel at a constant lengthwise depth, then, as discussed in Chapter 3, the nozzle can be moved back and forth across the channel width at variable velocity in the $x$-direction, while using a (much slower) constant scan speed in the length direction, $y$ (Figure 4-6). Ghobeity et al. [76] created wide (>6mm) flat pockets by oscillating the nozzle in this manner. However, in that case, the amplitude was so high that the source travelled at essentially a constant speed across the pocket width, yielding a virtually constant depth, i.e., the deceleration of the nozzle relative to the surface when changing direction could be neglected, and there was no need to solve the inverse problem to determine the required velocity. The present work considers the case where the velocity changes much more rapidly across the width, utilizing the nozzle acceleration to sculpt a desired cross-sectional shape. Since relatively small $(<1 \mathrm{~mm})$ features will be machined, the width of the source footprint and desired 
feature will be comparable, necessitating the use of inverse techniques to determine the source velocity for the desired shape.

Figure 4-6 shows the path taken by the centerline of a source as it moves at some velocity $v_{y}$ in the $y$ direction, while moving back and forth in the $x$ direction with an amplitude of $A$. It is noted that the motion need not be sinusoidal as in the W-shapes of Chapter 3; indeed, the waveform will control the resulting shape.

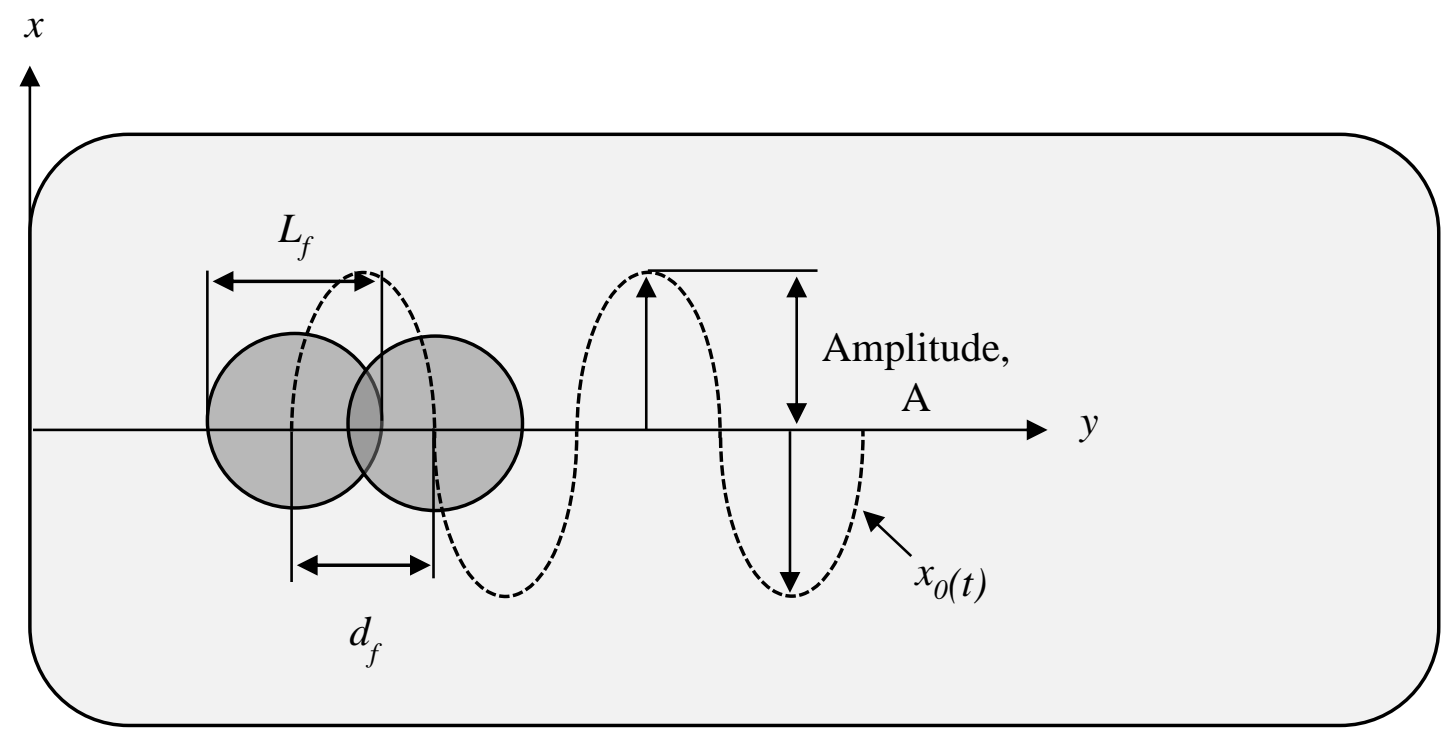

Figure 4-6 Schematic of foot prints of nozzle at the beginning and at half a period time span; the dashed line shows the path of oscillation.

\subsection{Determination of source erosive efficacy from profile of a shallow channel}

Equation (4.6) implies an $E_{s}$ that depends only on one spatial coordinate. For a source that only moves in the $y$ direction, i.e. machining a constant depth straight channel, Ghobeity et al. [31] showed that $E_{T}$ delivered to such a channel has the same functional form as the centerline erosive efficacy $\left(E_{s}\right)$ of a stationary source. This allowed surface evolution equations developed for holes to also be used for channels, i.e., the problem remained two-dimensional. Using a similar argument, it can be shown (Section 3.3.2) that for the present case where the source moves in two directions simultaneously the problem remains two dimensional. For example, if a source having 
a Guassian erosive efficacy (Eq. (4.6)) in the $x$ direction is moved at constant speed $v_{c}$ in the $y$ direction to create a channel of constant depth $z_{c}$, then the erosive efficacy anywhere except for the beginning or end of the scan will be approximately:

$$
E_{c}\left(x^{*}\right)=\frac{z_{c} v_{c}}{L_{f}} e^{-\beta^{2} x^{* 2}}
$$

where $\frac{z_{c} v_{c}}{L_{f}}$ is the average centerline erosive efficacy delivered over the footprint length $L_{f}$. The problem again is two-dimensional, and Eq. (4.17) can be used as the source erosive efficacy $E_{s}$ in Eq. (4.5) when it oscillates in the $y$ direction. If $z_{1}^{*}=\frac{z_{1}}{A}, x^{*}=\frac{x}{A}, v_{s}^{*}=\frac{v_{s}}{E_{c}(0)}, E_{T}^{*}=\frac{E_{T}}{E_{c}(0)}$, $T^{*}=\frac{T E_{c}(0)}{A}$ where $E_{c}(0)=\frac{z_{c} v_{c}}{L_{f}}$ then Eq. (4.5) becomes

$$
z_{0}^{*}=\int_{-1}^{1} \frac{e^{-\beta^{2}\left(x^{*}-x_{0}\right)^{2}}}{v_{s}^{*}\left(x_{0}{ }^{*}\right)} d x_{0}^{*}=E_{T}^{*} T^{*}
$$

\subsection{Expected shape of profile}

The expected resulting shape of the dimensionless profile, $z_{o}{ }^{*}$ due to the oscillation can be approximated by considering that, since the direction of movement changes abruptly at the end of the oscillation at $x^{*}= \pm 1$, then $v_{s}\left(x^{*}= \pm 1\right)=0$. Therefore, at the extremities of the movement, $z^{*}$ is expected to be of the same shape (but not magnitude) as the source efficacy. For a Gaussian source, this implies the periphery $y$ (i.e. for $x^{*>1}$ and $x^{*<-1)}$ follow the form of Eq. (4.6) with an appropriate multiplying factor, $C_{1}$. Furthermore, the finite width and rate of deceleration of the source as it approaches the periphery at $x^{*}= \pm 1$ and changes direction will determine the location and maximum magnitude of $z^{*}$. Assuming for a moment a point source, Eq. (4.15) implies $z^{*}$ should approach infinity at those points, i.e., where the instantaneous velocity of traverse is zero. The simplest general form for the profile in the region $-1 \leq x^{*} \leq 1$ satisfying this requirement is of 
order $1 /\left(1-x^{2}\right)^{n}$. In reality, since the source is of finite size, the profile $z^{*}$ will of course not be infinite at these points.

The eroded shape $z_{o}{ }^{*}$ one can thus expect from the oscillatory movement of a Gaussian source is shown in Figure 4-7, where the constant $C_{1}$ defining the height of the Gaussian at the periphery be determined by setting the profiles defined in (i) and (ii) equal to each other at the maximum occurring at $x^{*}= \pm D$ to obtain

$$
\begin{aligned}
& z_{o}^{*}\left(x^{*}\right)=\frac{C_{2}}{\left(1-x^{* 2}\right)^{n}}\left[H\left(x^{*}+D\right)-H\left(x^{*}-D\right)\right]+e^{-\beta^{2}\left(x^{*}-D\right)^{2}} \frac{C_{2}}{\left(1-D^{2}\right)^{n}}\left[H\left(x^{*}-D\right)\right]+ \\
& e^{-\beta^{2}\left(x^{*}+D\right)^{2}} \frac{C_{2}}{\left(1-D^{2}\right)^{n}}\left[H\left(-x^{*}-D\right)\right]
\end{aligned}
$$

where $C_{2}$ is the desired non-dimensional centerline depth. The location of the maximum $D$ is related to how quickly the deceleration of the source occurs as it approaches the periphery, and the factor $n$ controls the shape of the central portion, i.e. how quickly it increases as $x^{*}= \pm 1$ is approached. Equation (4.19) describes a family of possible desired 'W-shapes' that could be machined, should appropriate velocity functions be found. Such channels might find use, for example, in micro-particle sorting [67,88].

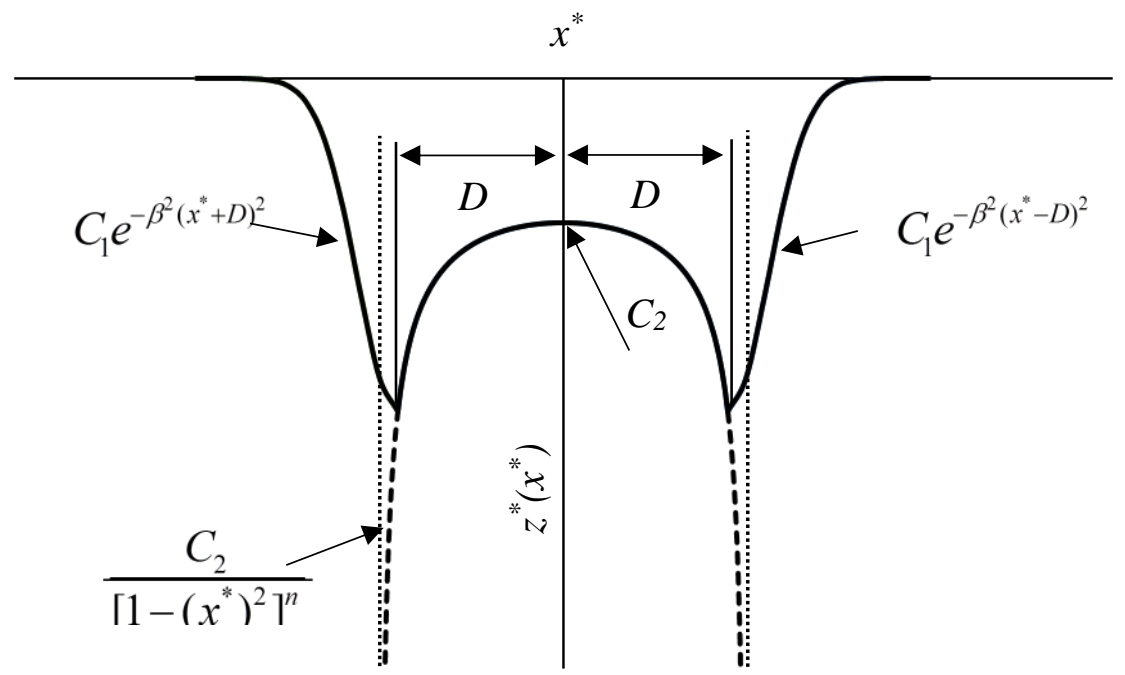

Figure 4-7 Expected shape of feature resulting from oscillation of a Gaussian source in range $1<\mathrm{x}^{*}<1$ 


\subsection{Determining the velocity function required to machine a desired $\mathrm{W}$ shape}

If the source gives a Gaussian kernel, since the bounds on the nozzle movement are finite (equal to the amplitude of oscillation), the infinite bounds convolution integral solution presented in Section 4.2.2.1 cannot be directly used. Nevertheless, if appropriate modifications are made, it can still be used to obtain a very good estimate of the oscillating velocity function. Assume for a moment that a Gaussian source moves, as shown in Figure 4-8, from $-\infty$ to $+\infty$ in the positive $x$ direction with a velocity $v_{s}{ }^{*}\left(x^{*}\right)+C$, where $v_{s}{ }^{*}\left(x^{*}\right)$ represents the non-uniform velocity to create the W-shaped feature within $-1<x^{*}<1$.

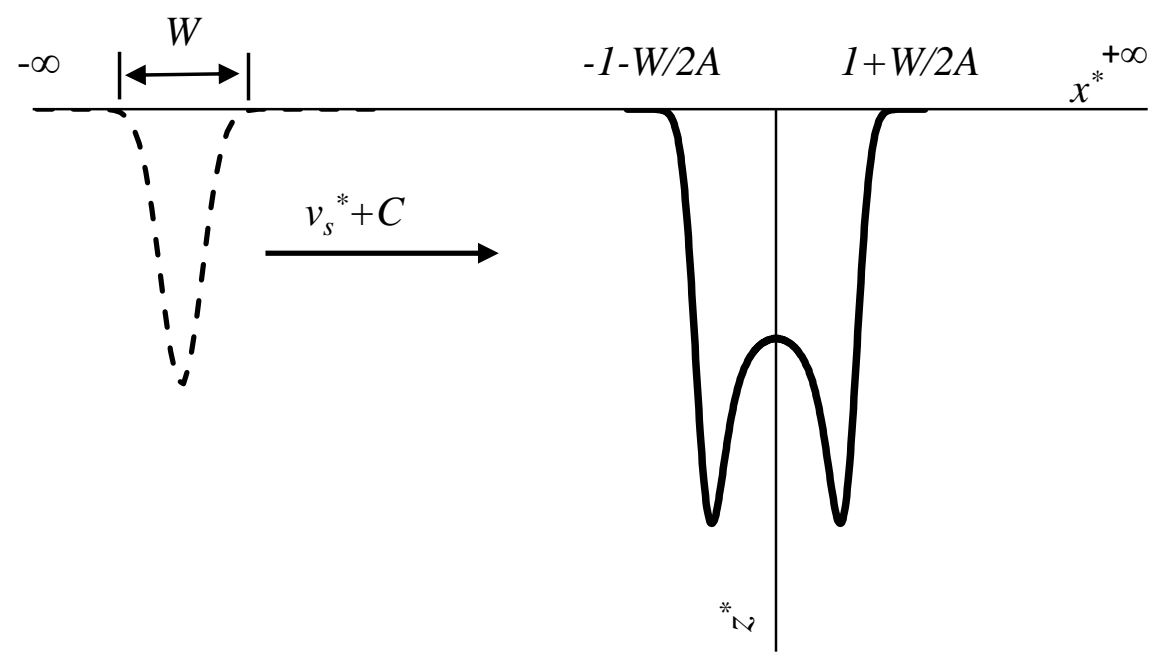

Figure 4-8 A Gaussian source (dashed line) moving in the $x$-direction with velocity $v_{s}^{*}+C$ to create the desired feature (solid line) centered at $x^{*}=0$ with bounds at $x^{*}=1+W / 2 A$ and $-1-W / 2 A . W$ is the width of the source.

The profile resulting from such a movement will be

$$
z^{*}=\int_{-\infty}^{\infty} \frac{e^{-\beta^{2}\left(x^{*}-x_{0}^{*}\right)^{2}}}{v_{s}^{*}\left(x_{0}^{*}\right)+\mathrm{C}} d x_{0}^{*}=\left(\int_{-\infty}^{-1} \frac{e^{-\beta^{2}\left(x^{*}-x_{0}^{*}\right)^{2}}}{v_{s}^{*}\left(x_{0}^{*}\right)+\mathrm{C}} d x_{0}^{*}+\int_{-1}^{1} \frac{e^{-\beta^{2}\left(x^{*}-x_{0}^{*}\right)^{2}}}{v_{s}^{*}\left(x_{0^{*}}\right)+\mathrm{C}} d x_{0}^{*}+\int_{1}^{\infty} \frac{e^{-\beta^{2}\left(x^{*}-x_{0}^{*}\right)^{2}}}{v_{s}^{*}\left(x_{0}^{*}\right)+\mathrm{C}} d x_{0}^{*}\right)
$$


As $C \rightarrow 0$, then the second term on the right hand side of Eq. (4.20) is the desired profile $z_{o}$ due to the oscillating source as it moves in the limited range $-1<x^{*}<1$. Since $v_{s}^{*}$ describes movement only for $-1<x^{* *}<1$, then $v_{s}{ }^{*}=0$ in the $1^{\text {st }}$ and $3^{\text {rd }}$ terms. Multiplying by $\frac{\beta}{\sqrt{\pi}}$, Eq. (4.20) thus becomes

$$
\frac{\beta}{\sqrt{\pi}} \int_{-\infty}^{\infty} \frac{e^{-\beta^{2}\left(x^{*}-x_{0}^{*}\right)^{2}}}{v_{s}^{*}\left(x_{0}^{*}\right)+C} d x_{0}^{*}=\frac{\beta}{\sqrt{\pi}}\left\{\frac { 1 } { 2 C } \frac { \sqrt { \pi } } { \beta } \left[1-\operatorname{erf}\left(\beta\left(x^{*}+1\right)\right]+z_{o}^{*}+\frac{1}{2 C} \frac{\sqrt{\pi}}{\beta}\left[1+\operatorname{erf}\left(\beta\left(x^{*}-1\right)\right]\right\}\right.\right.
$$

where $\operatorname{erf}()$ is the error function that arises when the Gaussian function is integrated.

Comparison of Eqs. (4.21) and (4.7) shows that the solution for $v_{s}^{*}$ in Eq. (4.8) can now be used with the right hand side of Eq. (4.21) in place of $\frac{\beta}{\sqrt{\pi}} z_{1}{ }^{*}$, and by making $C$ sufficiently small to approach the exact solution. Use of this solution to determine the oscillation velocity waveform necessary to create W-shaped channels will be demonstrated in Section 4.3.3.

\subsection{Application to various topographies}

In the following sections, the application of the inverse methods of Section 4.2 are illustrated for various classes of desired topographies. Chapter 5 will use the results of micro-machining experiments to assess the performance of these methods for many of the same topographies.

\subsubsection{Channels with desired texture along their length}

Table 4-1 shows examples of desired textures and their required velocity functions. In each case the desired texture profile function was initially expressed as the first 15 terms of a Fourier series, by finding the Fourier series coefficients from Eqs. (4.11), (4.12) and (4.13). These coefficients were used in Eq. (4.10) to predict the required velocity function for a Gaussian kernel of finite size (Figure 4-2). The calculated coefficients can be found in Appendix A. 
Table 4-1 Examples of desired non-dimensional microchannel textures and velocity functions necessary to produce them using method of Section 4.2.2.1. Gaussian source erosive efficacy with $\beta / z_{1}^{\min }=5.9 / 550 \mu \mathrm{m}=0.0107 \mu \mathrm{m}^{-1}$.

$\begin{array}{lccc}\text { Desired centerline } & \text { Desired shallow channel 3D } & \text { 2D profile of desired shallow channel } & \text { Required scan velocity: } v^{*}\left(y^{*}\right) \\ \text { texture: } & \text { topography: } z_{1}^{*}\left(x^{*}, y^{*}\right) & \text { centerline: } z_{1}^{*}\left(x^{*}=0, y^{*}\right) & \end{array}$
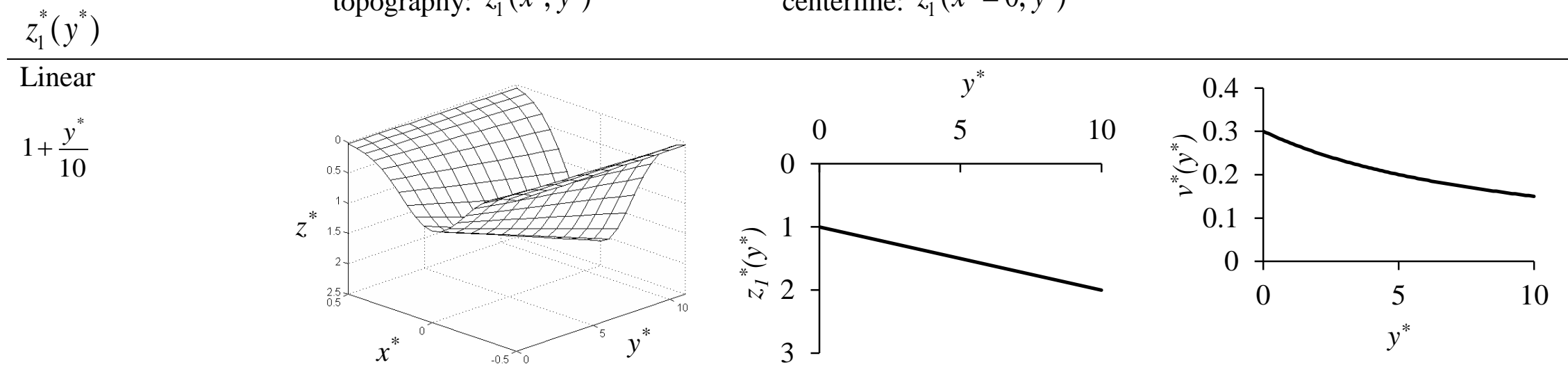

\section{Parabolic}

$$
-\frac{y^{* 2}}{25}+2 \frac{y^{*}}{5}+1
$$
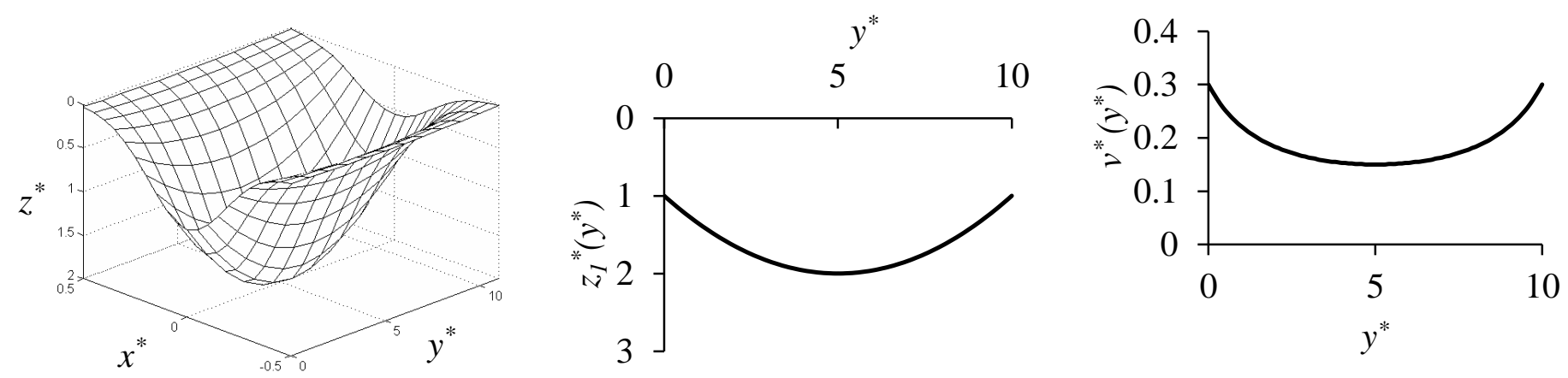
Sinusoidal

$\sin \left(\frac{2 \pi y^{*}}{10}\right)+2$

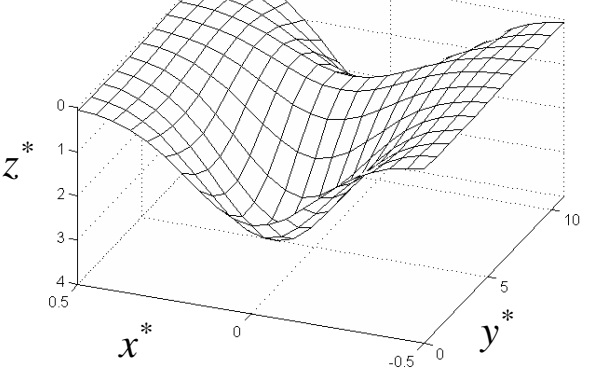

Half circle $-\sqrt{25-\left(y^{*}-5\right)^{2}}+6$

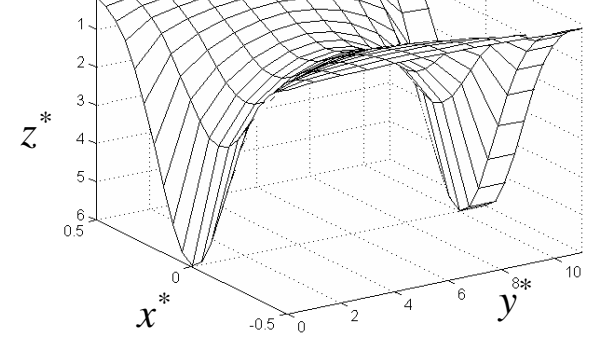

Inversed half circle $\sqrt{25-\left(y^{*}-5\right)^{2}}+1$

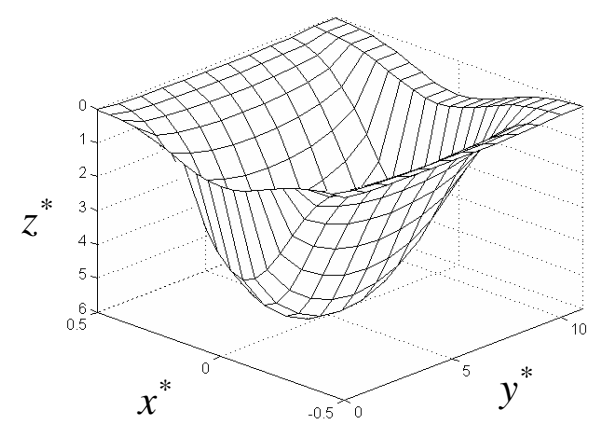

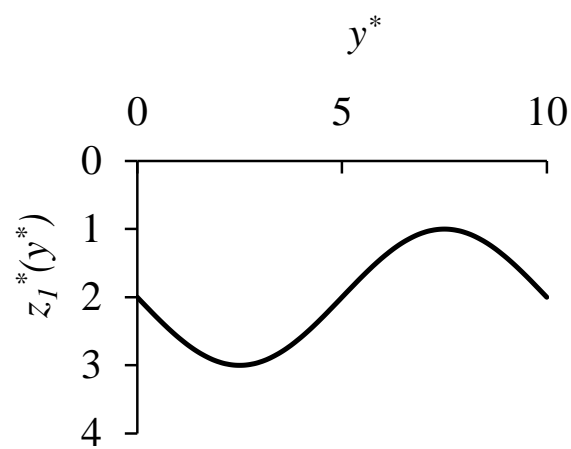
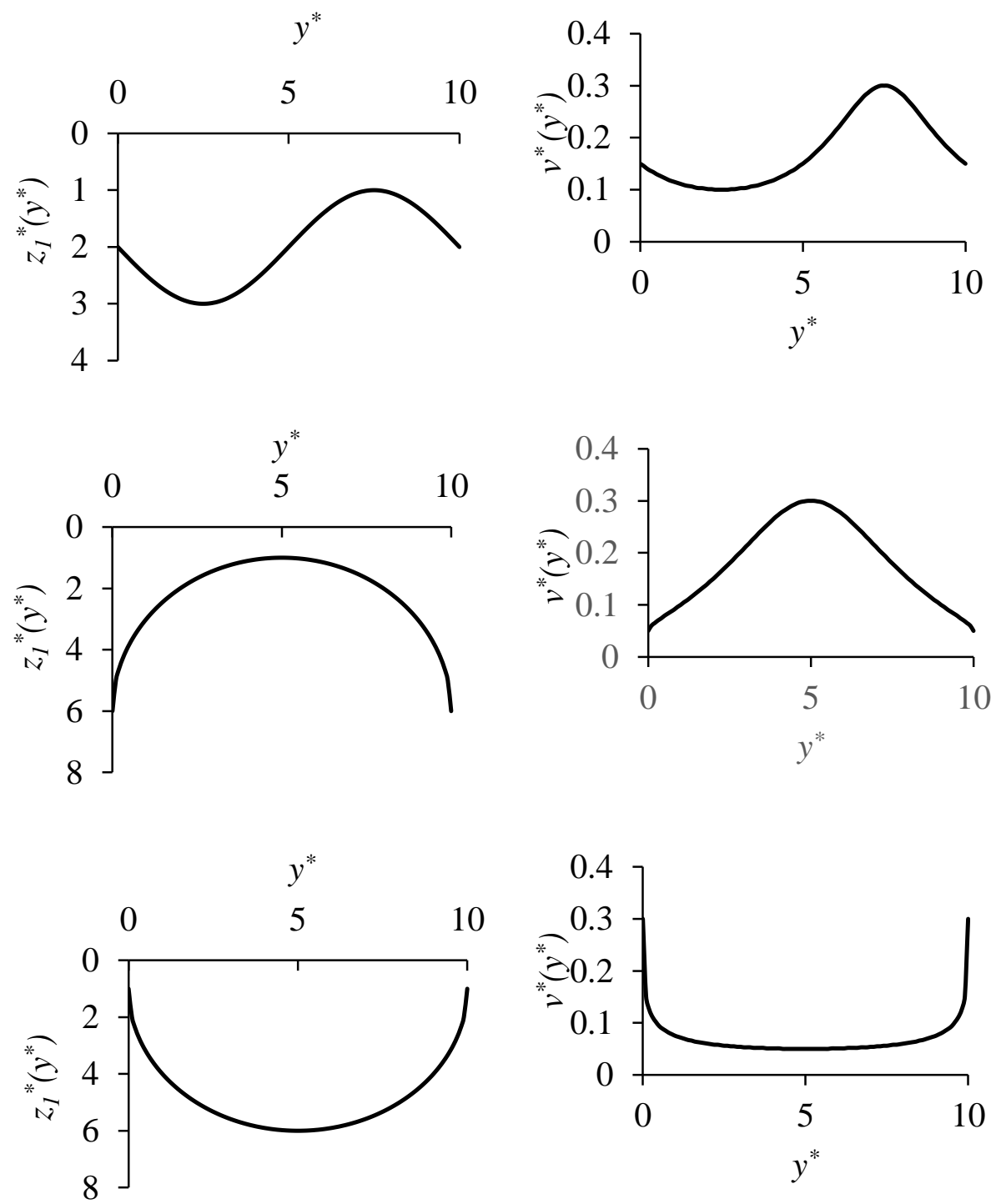
Since the feature sizes in Table 4-1 are relatively large, i.e. 5-10 times the source width, the point source solution in Eq. (4.15) could also have been used to obtain a very good approximation of the required velocity function. However, as the feature length becomes smaller (or the source larger), the point and Gaussian source solutions can deviate. For example, Figure 4-9 (circles) shows a desired sine wave of lower wavelength. The required velocities to machine this function using both Eqs. (4.10) (Gaussian source) and (4.15) (point source) are shown in Figure 4-10, where significant differences can be seen. If these velocity functions are used in Eq. (4.5) to predict the resulting shallow profiles (using appropriate conversion between dimensionless and dimensional quantities), Figure 4-9 shows that the point source solution significantly underestimates the amplitude, while the finite source solution matches the desired one. Experimental evidence for these differences will be presented in the Chapter 5.

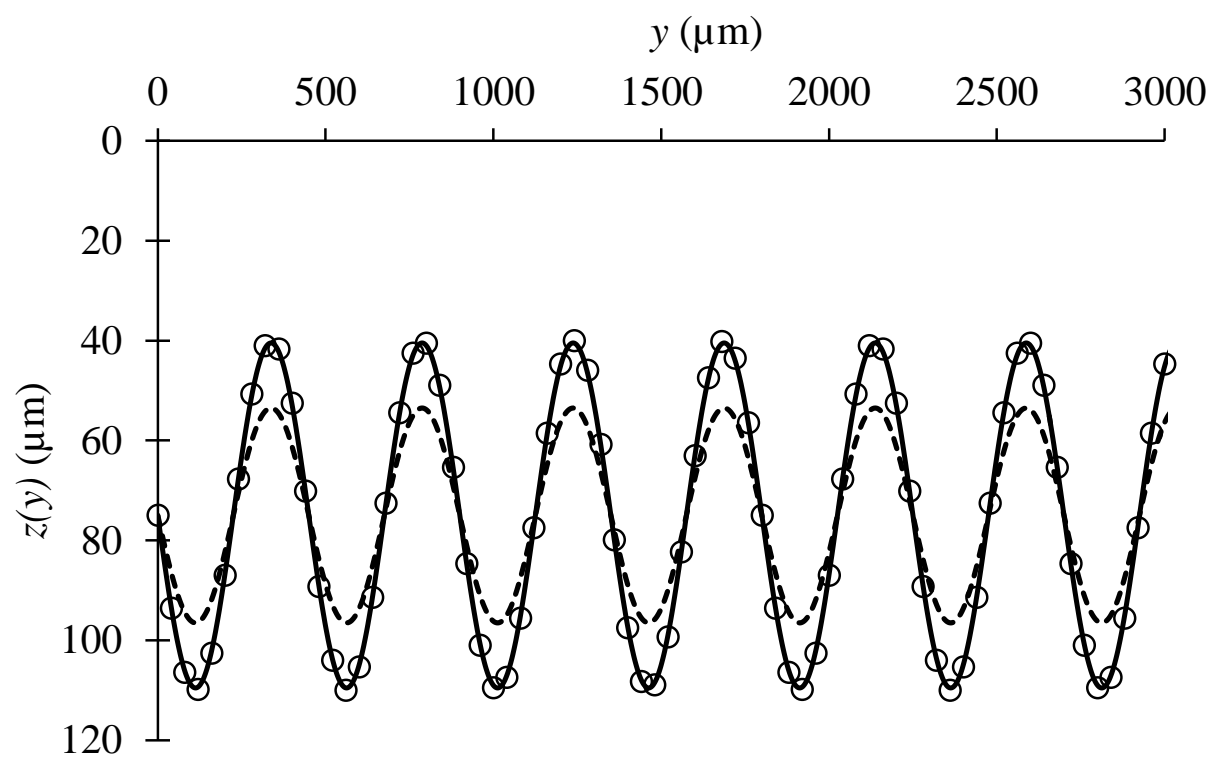

Figure 4-9 Predicted profiles using finite Gaussian (solid line) and point source (dashed line) solutions for a desired (circles, wavelength $=452 \mu \mathrm{m}$, amplitude $=35 \mu \mathrm{m}$ and $\beta=0.4$ ) sinusoidal texture of lower wavelength. It has been assumed that the point source strength, $K=z_{c} v_{c}=1$. 


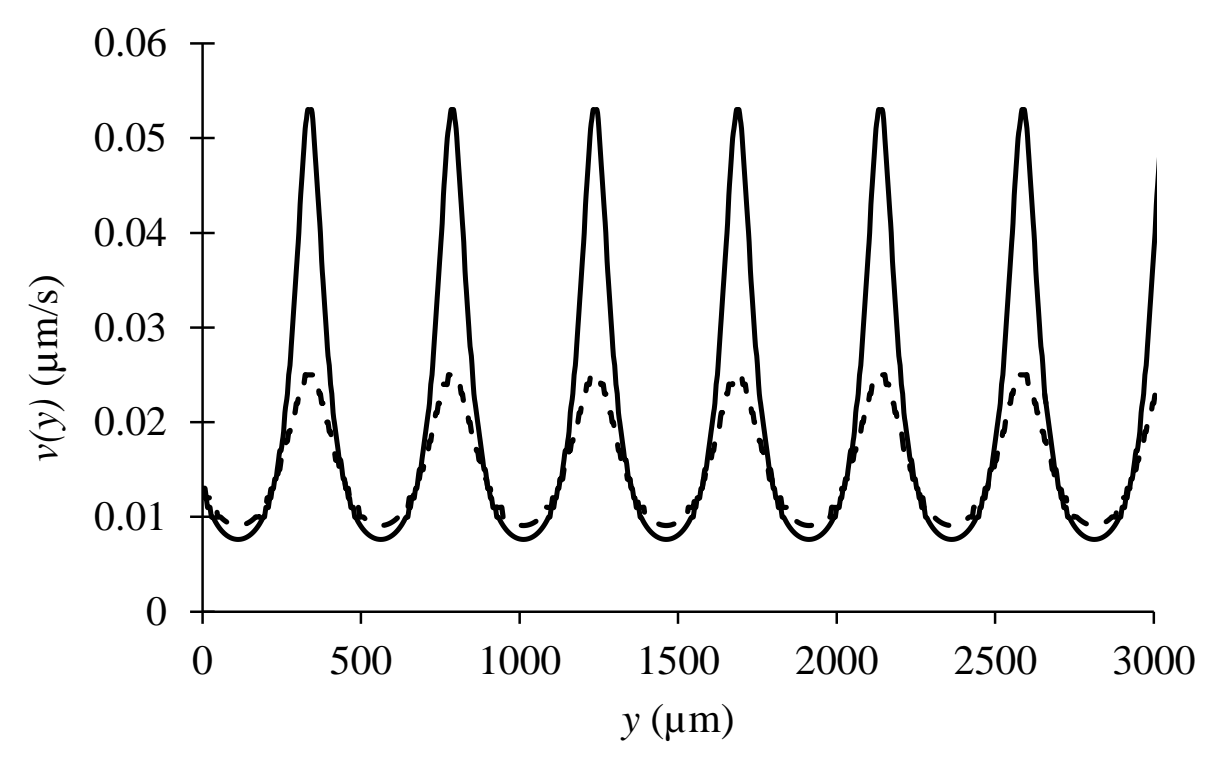

Figure 4-10 Predicted velocities to machine the desired profile of Figure 4-9 using Gaussian finite (solid line) and point source (dashed line) solutions to inverse problem. It has been assumed that the point source strength, $K=z_{c} v_{c}=1$.

In general, the error in the machined profile introduced by assuming a point source when a Gaussian source is actually used will increase as the source becomes larger ( $\beta$ decreases) and when the second and higher derivatives of $z_{d}{ }^{*}$ are significant. This error can be expressed as

$$
\frac{z_{1}^{*}\left(s^{*}\right)}{z_{p t}^{*}\left(s^{*}\right)}=1+\frac{\int_{s_{i}^{*}}^{s_{f}^{*}} e^{-\beta^{2}\left(s^{*}-s_{0}^{*}\right)^{2}} \sum_{j=1}^{\infty} \frac{1}{\mathrm{j} !}\left(\frac{-1}{4 \beta^{2}}\right)^{j} \frac{d^{2 j} z_{1}^{*}}{d s^{* 2 j}} d s_{0}{ }^{*}}{\int_{s_{i}^{*}}^{s_{f}^{*}} e^{-\beta^{2}\left(s-s_{0}\right)^{2}} z_{1}^{*} d s_{0}}
$$

where $z p t{ }^{*}$ is the profile that will result when a velocity predicted using the point source solution is used to machine the desired feature $z_{1}{ }^{*}$ by moving the source over the interval $s_{0}{ }^{*}=\left[s_{i}{ }^{*}, s_{f}{ }^{*}\right]$.

\subsubsection{Pockets with texture in two directions}

Assume that it is desired to machine a pocket with a repeating pattern that changes in the $y^{*}$ direction every $\Delta x^{*}=0.75$, i.e. at $x^{*}=0$, it is a cosine, at $x^{*}=0.75$ it is a positively sloped surface, at $x^{*}=1.5$ it is cosine and at $x^{*}=2.25$ it is a negatively sloped surface, where this pattern repeats. (Figure 4-11). 


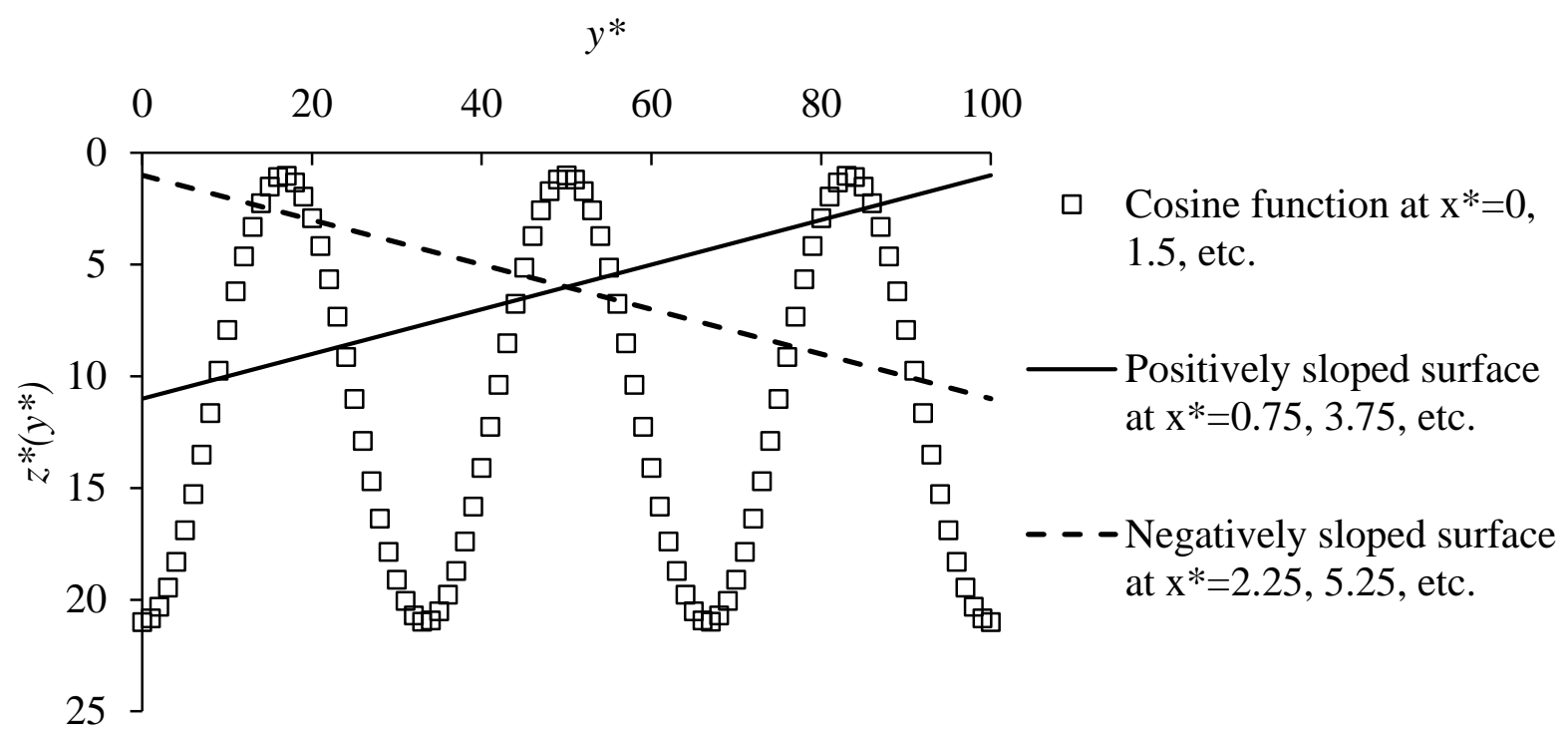

Figure 4-11 Desired patterns of $z^{*}\left(y^{*}\right)=11+10 \cos \left(6 \pi y^{*} / 100\right)$ (squares), $z^{*}\left(y^{*}\right)=11-y^{*} / 10$ (line) and $z^{*}\left(y^{*}\right)=1+y^{*} / 10$ (dashed line) that change every $\Delta x^{*}=0.75$.

Following the recommendation of section 4.2.4.2 the width of strips, $w_{s}$, considered to be 0.75 since the erosive efficacy of Figure 4-2 was used with $w_{e}=1$. The velocity functions necessary to machine the centerline of these strips (Figure 4-12) were found following the procedure of section 4.2.2.1 and by using Eq. (4.8). 


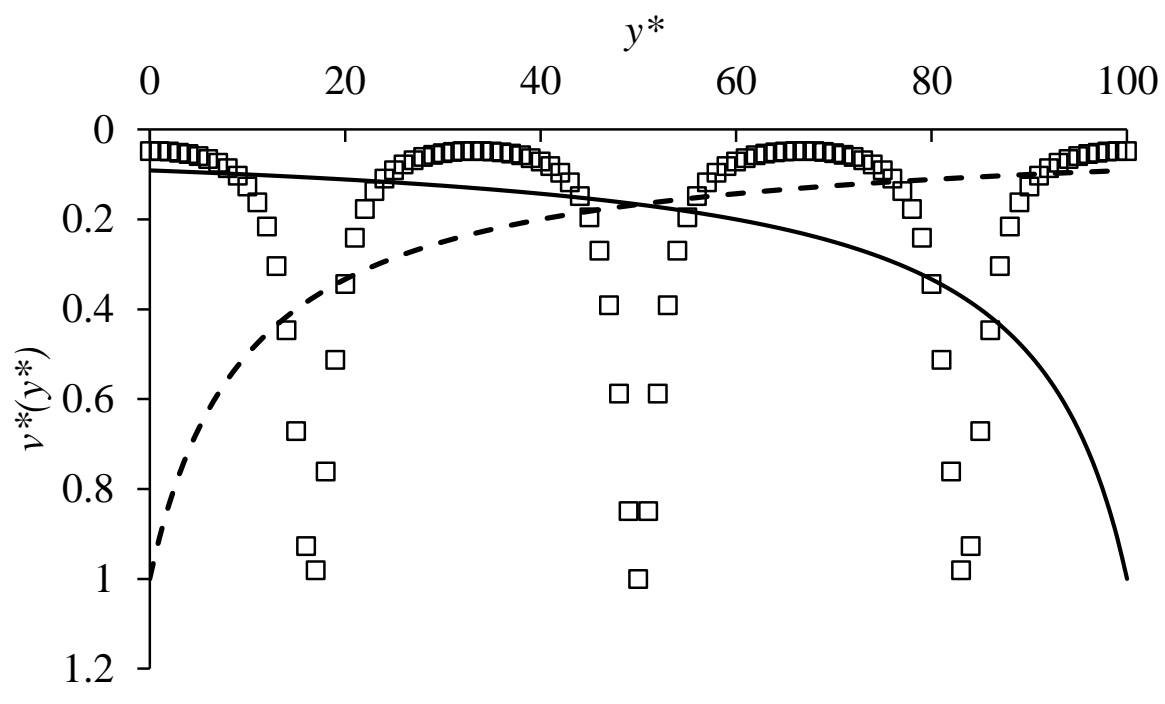

Figure 4-12 Velocity functions required to machine the profiles of Figure 4-11 (symbols match with the ones in Figure 4-11).

The profile of each strip based on this predicted velocity was calculated using Eq. (4.5). Using the procedure of section 4.2.4.2, the final predicted 3D profile is shown in Figure 4-13.

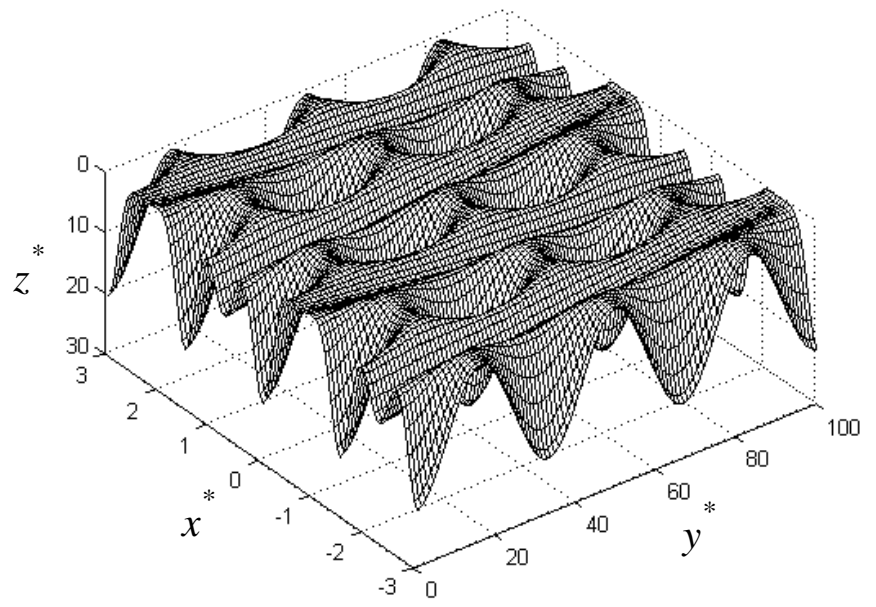

Figure 4-13 Predicted pocket with desire texture of Figure 4-11 using a Gaussian foot print with $\beta / z_{1}^{\text {min }}=3.89 / 50 \mu m=0.0778 \mu m^{-1}$ with strip width $w_{s}=0.75 w_{e}$ 
As discussed in section 4.2.4.2, Figure 4-14 shows that if the offset distance is below the suggested value of $0.75 w_{e}$, the erosive efficacies from adjacent strips will overlap to an extent that makes it impossible to create the desired texture.

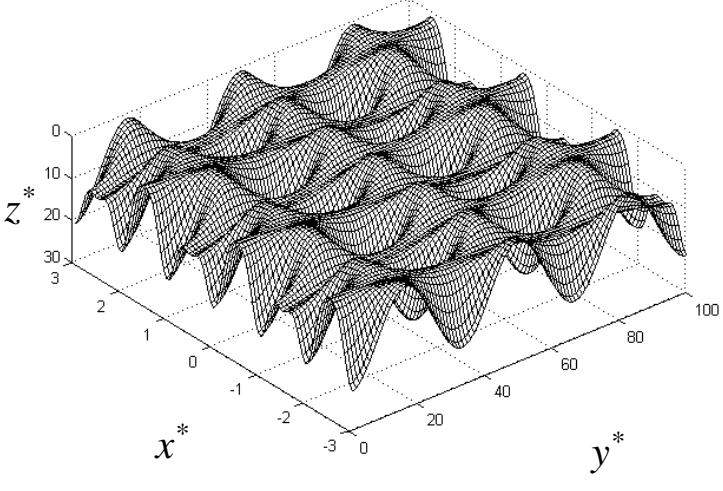

(a)

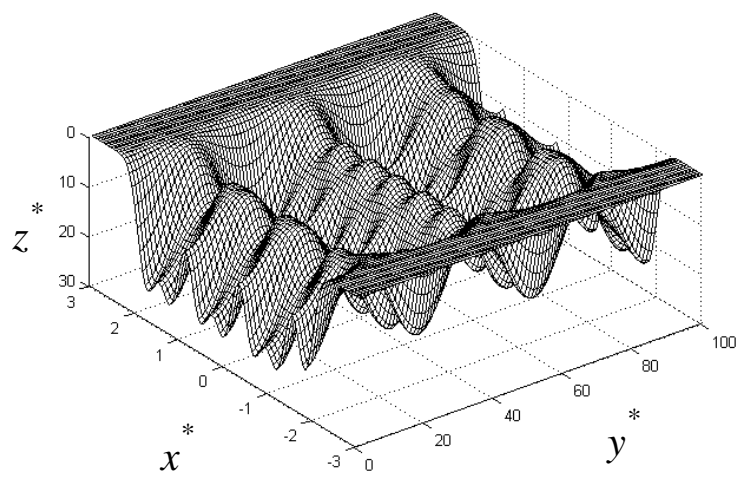

(c)

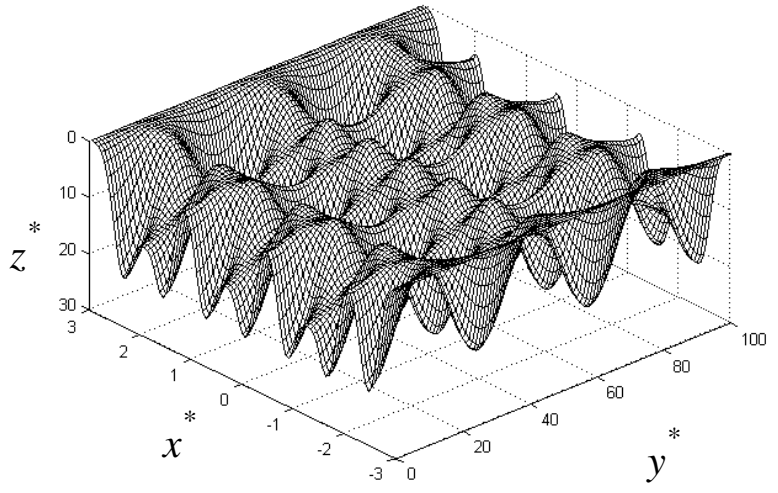

(b)

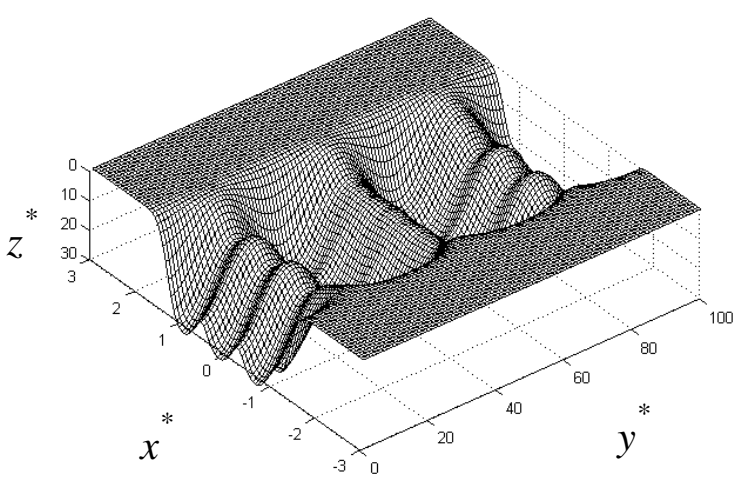

(d)

Figure 4-14 Predicted texture for strip to efficacy width ratios $w_{s} / w_{e}$ of (a) 0.5 , (b) 0.4 (c) 0.3 and (d) 0.2. As the offset becomes smaller, the pattern deviates from the desired one.

\subsubsection{Channels with prescribed cross sectional shape}

Assume that it is desired to obtain a micro-channel of constant depth in the $y$ direction, with a W-shaped cross section (Figure 4-15) having $n=1 / 2, D=0.9$, and $C_{2}=0.5$ (Eq. (4.19)) using a Gaussian source with $\beta / A=4.25 / 500=0.0085 \mu m^{-1}$. 


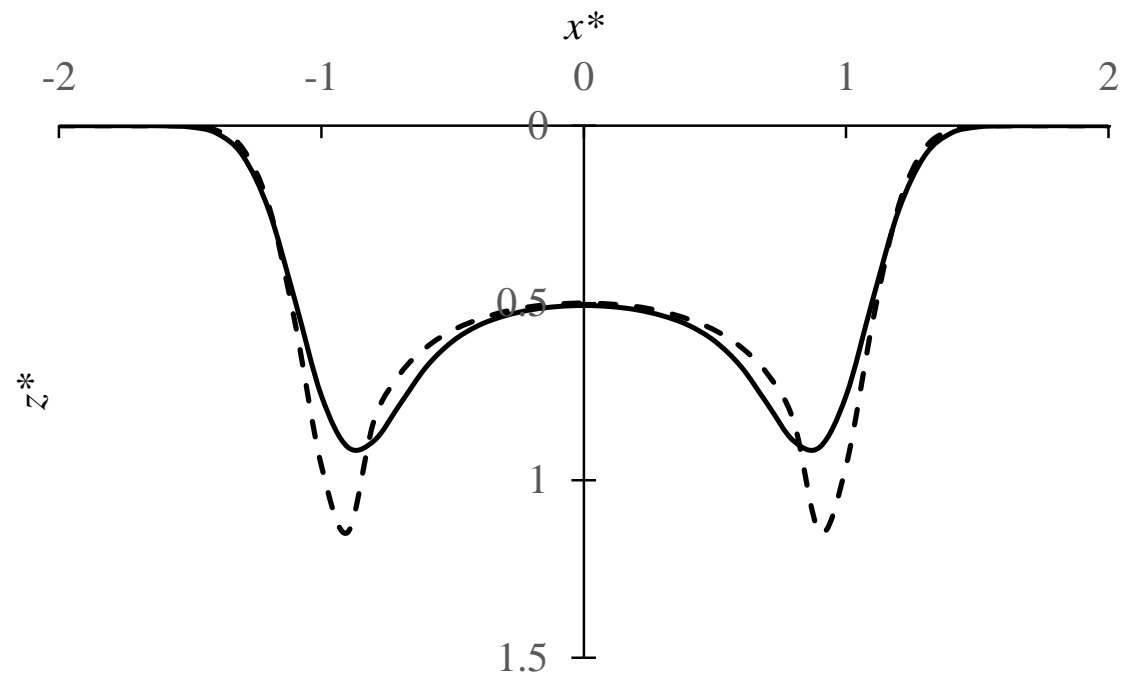

Figure 4-15 Desired W-shaped channel cross section with $n=1 / 2, D=0.9$, and $C_{2}=0.5$ (dashed line) and that predicted to be machined using Gaussian source $\left(\beta / A=0.0085 \mu \mathrm{m}^{-1}\right)$ with $v_{s}{ }^{*}=$ $0.834 \sqrt{1-x_{0}^{* 2}}$ (line).

The method of Section 4.2.4.3 is appropriate in this case, i.e., Eq. (4.19) is used to define $z_{0}^{*}$ in Eq. (4.21). Then, using the right hand side of Eq. (4.21) as the left hand side of Eq. (4.7), the solution to Eq. (4.10) for $v_{s}$ can be obtained, and is shown in Figure 4-16.

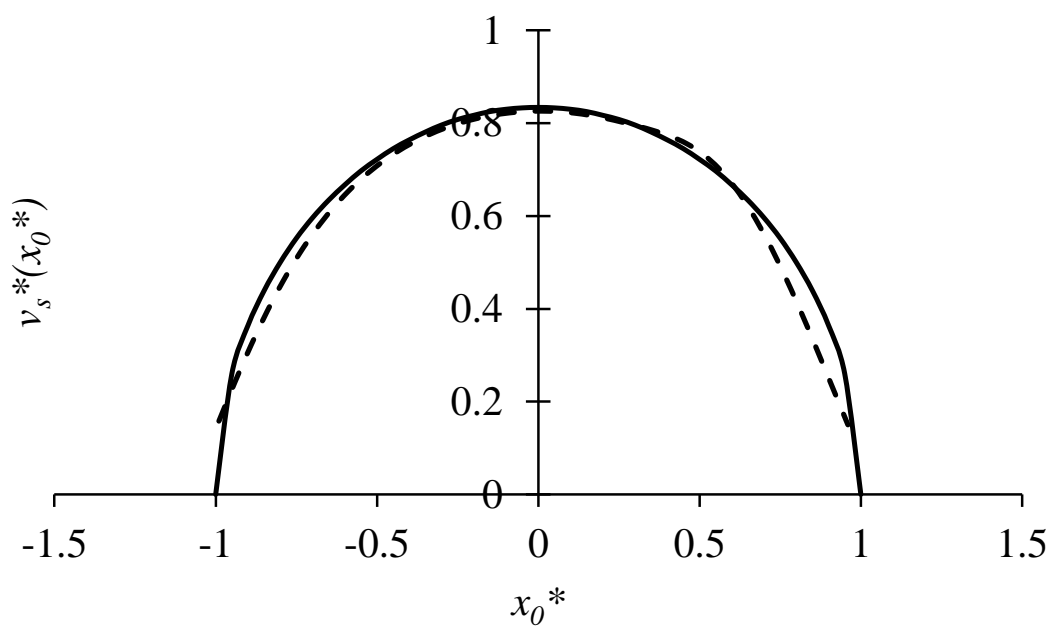

Figure 4-16 The predicted required velocity function to machine the $\mathrm{W}$ - shaped channel with $\mathrm{n}=1 / 2$ and $\mathrm{D}=0.9$ (dashed line) in Figure 4-15. Solid line represents $v_{s}{ }^{*}=0.834 \sqrt{1-x_{0}^{* 2}}$ (line). 
As can be seen in Figure 4-16, the solution approaches

$$
v_{s}^{*}\left(x_{0}^{*}\right)=\frac{\sqrt{\pi}}{\beta C_{2}} \sqrt{1-x_{0}^{* 2}}
$$

for a small value of $C$ in Eq. (4.20). When transferred to the time domain using $v_{s}{ }^{*}=d x_{0}{ }^{*} / d t^{*}$, and enforcing $x_{0}{ }^{*}=-1$ at $t^{*}=0$, this is immediately recognized as

$$
x_{s}^{*}\left(t^{*}\right)=-\cos \left(\frac{\sqrt{\pi}}{\beta C_{2}} t^{*}\right)
$$

Thus, the sinusoidal source motion used to produce the $\mathrm{W}$-shapes in Chapter 3 corresponds closely to the desired feature shape with $\mathrm{D}=0.9$ and $n=1 / 2$. Figure 4-15 compares the predicted cross sections machined using the two velocity functions in Figure 4-16, i.e, the result of equation (4.5) with these two velocity functions. As it can be seen the two profiles are very close.

\subsection{Summary}

Novel inverse methodologies were introduced to predict the instantaneous source velocity functions required to micro-machine a wide variety of desired shallow feature profiles into brittle and ductile targets using gradient-etching AJM. It was demonstrated that the inverse problem can be expressed as a Fredholm integral equation of the first kind. Assuming motion in a single direction of a typical AJM source having a Gaussian erosive efficacy distribution, it was demonstrated that the equation can be solved in closed form using the theory of Gauss transforms that take as input a Fourier series representation of the desired eroded profile. Such movement was shown to be useful for creating surface texture. For oscillating movement of the source, useful for sculpting microchannel cross sectional shape, an approximate solution was presented. For nonGaussian sources, an approximate approach assuming a point source must be used. Once the velocity functions to create shallow profiles are obtained, predictions of deeper ones can be obtained using existing surface evolution models. 
Since any profile can in principle be expressed as a Fourier series, the inverse methodology was demonstrated by example to have the potential to be used in a wide variety of applications, including sculpting desired channel cross-sectional shapes, lengthwise micro-channel texture, pocket texture, and combinations. The limits of applicability of the methodologies in terms of, e.g. relative source and feature size, were also established. Finally, methodologies for recreating visual representations of $2 \mathrm{D}$ and $3 \mathrm{D}$ textures were presented. To the knowledge of the authors, this is the first time inverse methods have been used for path planning in AJM operations. In Chapter 5, the velocities predicted by the presented methodologies will be used to micro-machine a variety of desired features, and the resulting profiles will be compared to the originally desired ones, in order to validate the approach. 


\section{Chapter 5 Experimental verification of inverse methods to gradient etch three-dimensional features with prescribed topographies}

This chapter is based on the following published paper:

M. R. Sookhak Lari, M. Teti, and M. Papini. "Inverse methods to gradient etch three-dimensional features with prescribed topographies using abrasive jet micro-machining: Part II - Verification with micro-machining experiments" Precision Engineering, vol. 45, pp. 262-271, 2016.

\subsection{Introduction}

As reviewed in detail in Chapter 4, the topography of micro-channels in micro-fluidic devices can be changed using gradient etching techniques, which may allow desirable changes in the flow characteristics that, in turn, can affect the thermal and pressure distribution and dissipation. As also discussed in Chapter 4, although gradient etching has numerous applications, its three-dimensional nature limits the use of traditional micro-fabrication technologies. Moreover, there is a lack of methodologies that allow determination of the process parameters required to machine a particular desired feature topography.

Chapter 4 presented analytical inverse techniques that could be used to predict the velocity functions required to machine desired topographies using abrasive jet micro-machining (AJM), a nontraditional microfabrication technology. Case studies performed in Chapter 4 established the promise of these techniques for machining a wide variety of three-dimensional topographies. This chapter presents experimental data that verifies the use of these models for machining channels and pockets having various prescribed surface textures and cross-sectional shapes.

\subsection{Experiments}

As explained in the following sections, the inverse techniques of the Chapter 4 were used to determine, for a given erosive source efficacy, the velocity functions required to machine a variety of prescribed shallow-depth feature topographies, and then the features were machined using these functions. For cases where relatively deep features were desired, the predicted velocity 
functions were used to determine the resulting erosive efficacies, and existing surface evolution equations were then used to predict the deeper feature topographies.

The etched 3D topography of the machined micro-channels and pockets were measured using an optical profilometer (Nanovea ST400 Micro Photonics Inc., Irvine, CA, USA), which had a depth resolution of $25 \mathrm{~nm}$ and a lateral resolution of $0.1 \mu \mathrm{m}$. Multiple scans along the feature length were performed at locations separated by $30 \mu \mathrm{m}$ along the feature width. The step size between data points along the feature length was $5 \mu \mathrm{m}$. The resulting multiple $2 \mathrm{D}$ profiles were then plotted to obtain the 3D shape. Each reported profile represented the average of 15 data sets, i.e., three separately repeated repetitions and five profiles along the length of each channel. The measured machined topographies that had an average depth closest to the average of those repeats were compared to the originally desired topographies. The maximum difference in depths for the profiles within a given channel, and between repeats of different channels was typically less than $5 \%$. The measured topographies resulting from machining with the predicted velocity functions were then compared to those that were originally desired.

All profiles and velocity functions were presented in their dimensional form, using appropriate conversion from the dimensionless quantities presented in Chapter 4.

\subsubsection{Micro-machining apparatus}

An AccuFlo AF10 Micro-Abrasive Blaster, (Comco, Inc. Burbank, CA, USA) operating at $200 \mathrm{kPa}$ air pressure was used for all experiments. Two programmable computer-controlled linear stages (Aerotech Inc., Pittsburgh, PA, USA) with positioning resolutions in two independent perpendicular directions of $0.5 \mu \mathrm{m}$ were used to move the target relative to the stationary nozzle with various position-dependent velocities. More details on the experimental setup can be found in Chapter 2. Borosilicate glass (Borofloat, Swift glass Co. Inc., Elmira, NY, USA), and polymethylmethacrylate (PMMA) (type ACRYLITE ${ }^{\circledR}$ FF, CYRO Industries, Rockaway, NJ, USA) target materials were used to represent brittle and ductile, respectively, erosive systems. The abrasive powder was aluminum oxide $\left(\mathrm{Al}_{2} \mathrm{O}_{3}\right)$ (Comco, Inc. Burbank, CA, USA) of $\sim 25 \mu \mathrm{m}$ nominal diameter.

Two different direct-write AJM techniques were evaluated to avoid the need for attaching patterned masks to the surfaces. In the first, the previously developed adjustable shadow-mask of 
Chapter 2 was used. The system utilizes adjustable masks which are attached to a rectangular nozzle $(0.3 \mathrm{~mm}$ by $3.8 \mathrm{~mm})$ and made to contact the surface, in order to create a jet footprint whose width can be controlled. Previous work utilized the shadow mask to machine single pass straight channels, which exposed the mild steel mask to relatively low particle doses. Use of multiple passes at various traverse velocities in the present work, however, exposed the mask to much higher particle doses, and the mild steel masks were found to fail before the machining experiments could be completed. A number of metal mask materials (tungsten carbide, spring steels, tool steels and copper) were evaluated, but tungsten carbide was found to erode between 2-4 times slower than any of them, so tungsten carbide was used in all of the present experiments. The mask opening was controlled in the range of $150-450 \mu \mathrm{m}$ by using feeler gauges. The nozzle to surface standoff for the shadow mask machining experiments was kept constant at $20 \mathrm{~mm}$ while the mask to surface distance was $<100 \mu \mathrm{m}$. The erosive efficacy distribution through the shadow mask was typically Gaussian [89]. In the second technique, a much smaller $0.46 \mathrm{~mm}$ round nozzle was used with no mask, and with a very small (100 $\mu \mathrm{m}$, controlled using a feeler gauge) nozzle to surface standoff distance, in order to limit the footprint of the jet to between 500 and $570 \mu \mathrm{m}$. The erosive efficacy in this case was non-Gaussian. For both maskless machining and machining with the shadow mask, the jet was incident perpendicular to the target surface. The mass flow rate at the nozzle exit for the maskless and shadow mask techniques (larger rectangular nozzle) were $2.8 \mathrm{~g} / \mathrm{min}$ and 5.6 $\mathrm{g} / \mathrm{min}$ respectively. As was suggested in Chapter 4, Gaussian and spline curve fits of the measured profiles of shallow (aspect ratio < 0.1) constant-depth single pass channels were used to characterize the shadow mask and maskless source erosive efficacies, respectively.

\subsubsection{Effect of machining step size}

The computer controlled stages could move at a constant velocity during each motion step. Thus, in order to be able to move the stage with a given velocity function, the function was discretized into small segments, each of which had a different constant velocity. It is clear that as the segments, or steps, become smaller, the generated velocity function will be closer to the intended one and the accuracy will be higher. The lower limit of step size was $0.5 \mu \mathrm{m}$ based on the resolution of the stage. The upper limit for the step size depended on the nozzle footprint of the nozzle and the resolution of the feature to be machined. 
For the footprint $(\sim 200-550 \mu \mathrm{m})$ and feature sizes $(\sim 250 \mu \mathrm{m}-5 \mathrm{~mm})$ considered in the present work, it was found that a $50 \mu \mathrm{m}$ step was adequate to reproduce the velocity functions faithfully and thus not produce undesired texture. Although the stage was able to generate much smaller step sizes, the accuracy was negatively affected because of the finite time necessary to accelerate the stage to the desired velocity within a given step.

\subsubsection{Micro-machining channels with desired texture along their length}

To demonstrate the flexibility of AJM to gradient etch texture into micro-channels, straight micro-channels with depths that changed linearly, parabolically, and sinusoidally along their depth were machined in glass using the maskless technique at a standoff of $100 \mu \mathrm{m}$ by continuously varying the speed at which the target moved relative to the stationary nozzle, using the velocity functions predicted by the point source solution of Section 4.2.2.2. Visualizations of the full desired and measured 3D profiles were constructed using the methods of Section 4.2.4.1, and compared. The linearly varying depth channels were machined at slopes varying between 2 and 6 \%for depths between $30 \mu \mathrm{m}$ and $200 \mu \mathrm{m}$. The micro-channels that had depths that varied parabolically had a maximum depth between $140 \mu \mathrm{m}$ and $250 \mu \mathrm{m}$ over a length of $5 \mathrm{~mm}$. The sinusoidally varying depth micro-channels were machined at a maximum depth of $230 \mu \mathrm{m}$ with an amplitude of $181 \mu \mathrm{m}$, wavelengths between 1.7 and $5 \mathrm{~mm}$, and widths between $506 \mu \mathrm{m}$ and $515 \mu \mathrm{m}$.

\subsubsection{Pockets with texture in two directions}

Using the maskless technique at a standoff of $100 \mu \mathrm{m}$, pockets with different prescribed sinusoidal patterns in two perpendicular directions were machined in glass using multiple overlapping adjacent passes, as described in Section 4.2.4.2. The point source inverse solution provided the required velocities for each offset pass. The visualization of the full desired and measured 3D profiles were also constructed and compared. 


\subsection{Results and discussion}

\subsubsection{Source erosive efficacy}

As suggested in Chapter 4, the source erosive efficacies resulting from AJM can either be Gaussian or non-Gaussian, depending on the type of source. Figure 5-1 shows typical erosive efficacies resulting from the shadow-mask (Gaussian) and maskless at a small standoff (nonGaussian) sources used in this chapter. These were obtained directly from spline (maskless) and Gaussian (shadow mask) curve fits of the eroded profiles of shallow channels machined at a constant velocity, as explained in Section 5.2.1. The equivalent point source strengths, $K=z_{c} v_{c}$, where $z_{c}$ and $v_{c}$ are, respectively, the shallow channel centerline depth and scan velocity used to create it, were between $75 \times 10^{3}-128 \times 10^{3}{\mu m^{2}}^{2} \mathrm{~s}$.

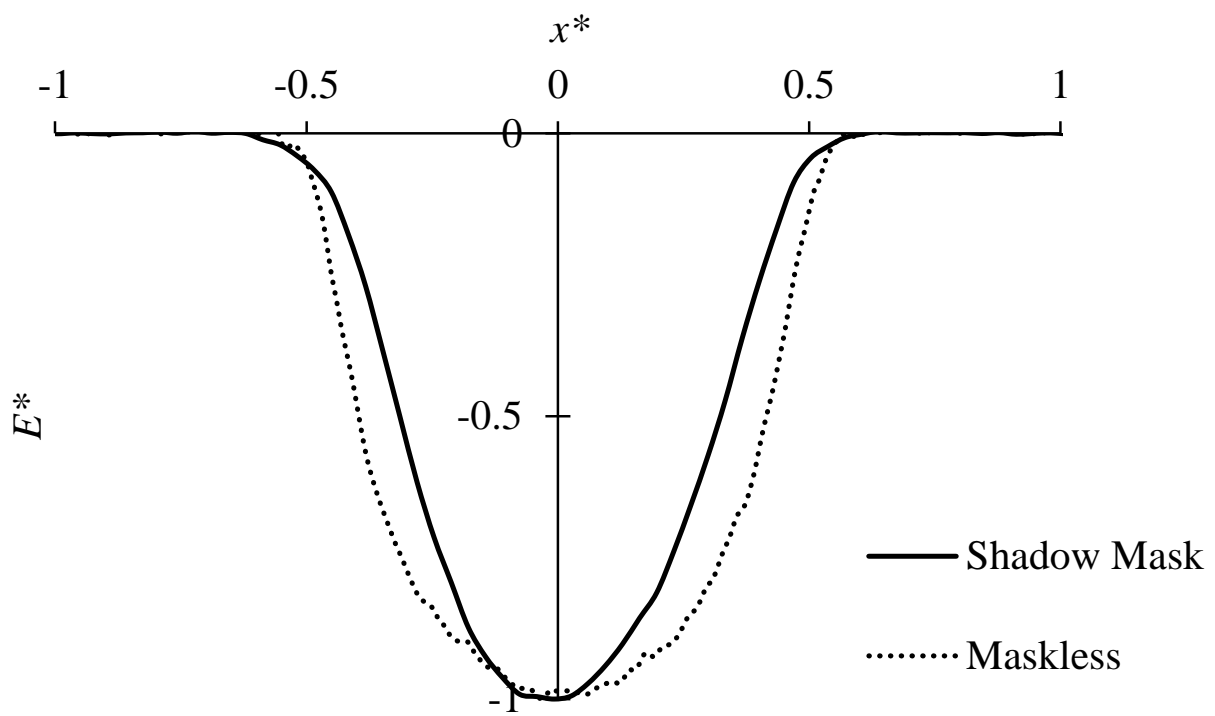

Figure 5-1 Erosive efficacies of shadow mask and maskless nozzle, normalized in width, $W$, by $411 \mu \mathrm{m}$ and $515 \mu \mathrm{m}$ respectively, and in depth by the value of erosive efficacy at $x=0$. For the

$$
\text { shadow mask, } E^{*}\left(x^{*}\right)=e^{-\left(\beta x^{*}\right)^{2}} \text { with } x^{*}=\frac{x}{411 \mu m} \text { and } \beta=3.873 \text {. }
$$

\subsubsection{Micro-machining of channels with desired texture along their length}

As explained in Section 5.2.3, gradient etched channels whose depth changed in a variety of prescribed ways were machined using the velocities predicted using the inverse methods of Chapter 4. In the following sections, the measured profiles are compared to the originally 
prescribed (desired) ones. In addition, since a point source solution to the inverse problem was used to predict the velocity, where in reality a finite source was used to machine the channels, the 'predicted' profile is also presented. This predicted profile results from using the point-source predicted velocity, $v_{s}(y)$ and the actual source erosive efficacy $E_{s}(y)$ in Eq. (4.5), i.e. $z_{1}(y)=\int_{y_{i}}^{y_{f}} E_{s}\left(y-y_{0}\right) / v_{s}\left(y_{0}\right) d y_{0}$. The difference between the predicted and desired profiles thus represents the expected error introduced by assuming a point source. The error is small, because, as discussed in Chapter 4, the feature length is large compared to the source. A case where the error is not small is presented in Section 5.3.2.4.

\subsubsection{Predicted and measured channels with linearly changing depth}

Figure 5-2 (a) (squares) shows an example of a desired centerline micro-channel profile that changes linearly according to $z_{1}(y)=51+128 y / 5000 \mu \mathrm{m}$. The predicted velocity function needed to produce it is shown in Figure 5-2(b), and the resulting measured profile machined using this velocity is shown by the circles in Figure 5-2(a). The solid line in Figure 4-3(a) represents the predicted profile using the predicted velocity. Figure 5-3 shows the predicted and measured 3D channels. The slope and depth of the measured channel were within $3.2 \%$ and $5.6 \%$ respectively, of that desired, indicating that the inverse method solution was able to accurately predict the velocity required to machine the desired linear gradient etch topography. 

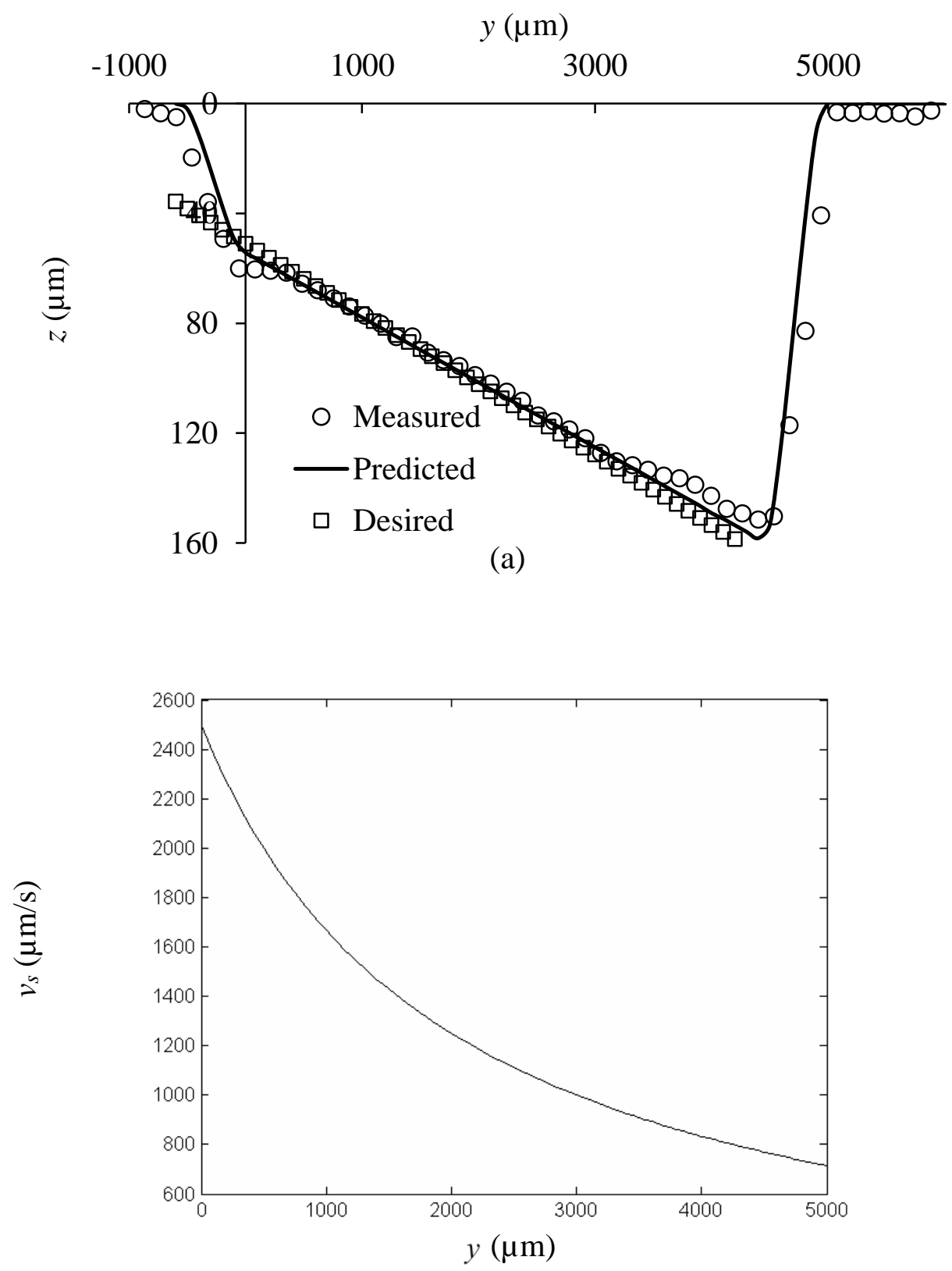

(b)

Figure 5-2 (a) Desired, predicted, and measured channel centerline profiles for a desired linear depth of $z_{1}(y)=51+128 y / 5000 \mu \mathrm{m}$; (b) predicted velocity function required to machine the desired profile. A maskless source was used with an equivalent point source strength of

$$
K=z_{c} v_{c}=128 \times 10^{3} \mu m^{2} / s .
$$




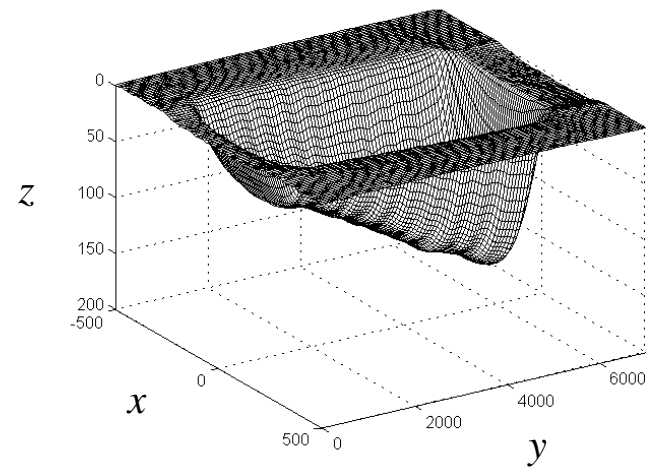

(a)

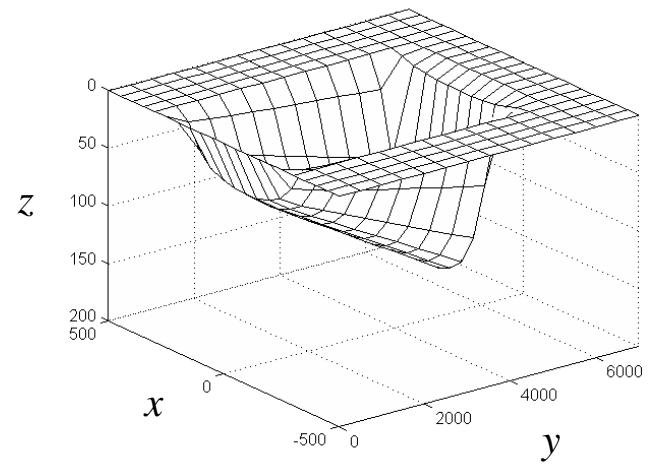

(b)

Figure 5-3 (a) Measured and (b) predicted 3D profile for channel of Figure 5-1. Dimensions in $\mu \mathrm{m}$.

\subsubsection{Predicted and measured channels with depth changing parabolically}

Figures 5-4 and 5-5 show that the inverse method solution was able to accurately predict the velocity required to machine a micro-channel whose desired profile varies parabolically as $z_{1}(y)=-490(y / 5000)^{2}+490 y / 5000+49 \mu \mathrm{m}$. The point-by-point average difference between the measured and desired channel depth was less than $5.9 \%$.

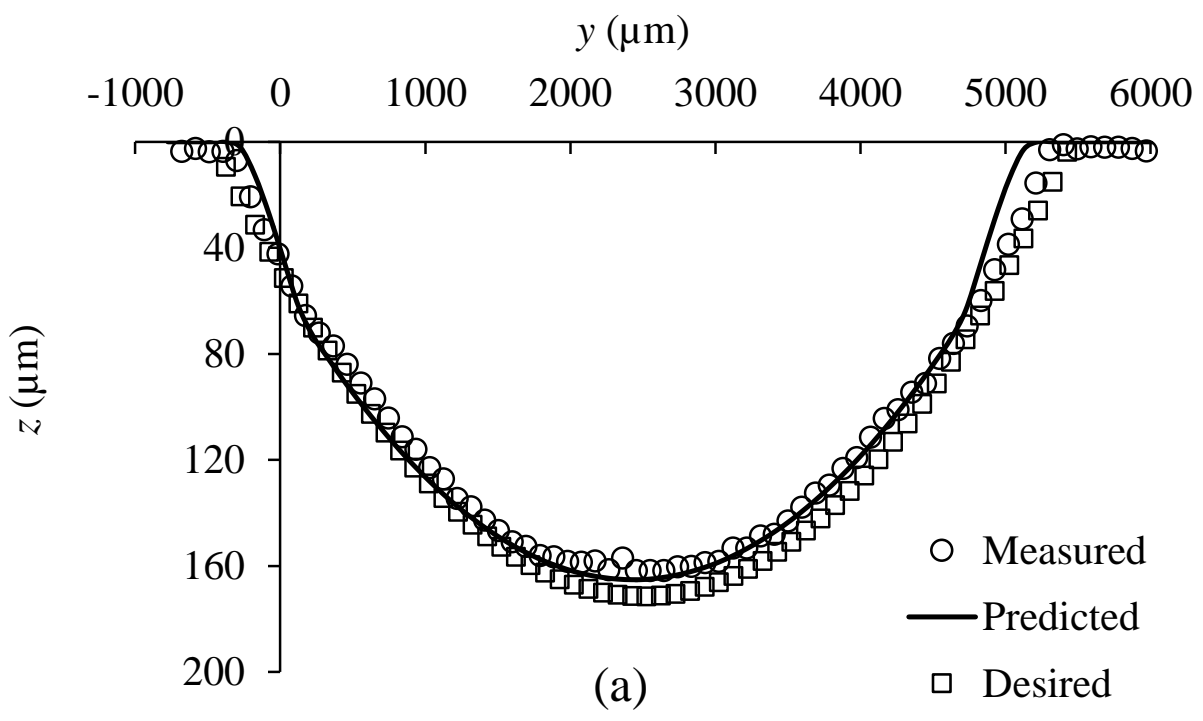




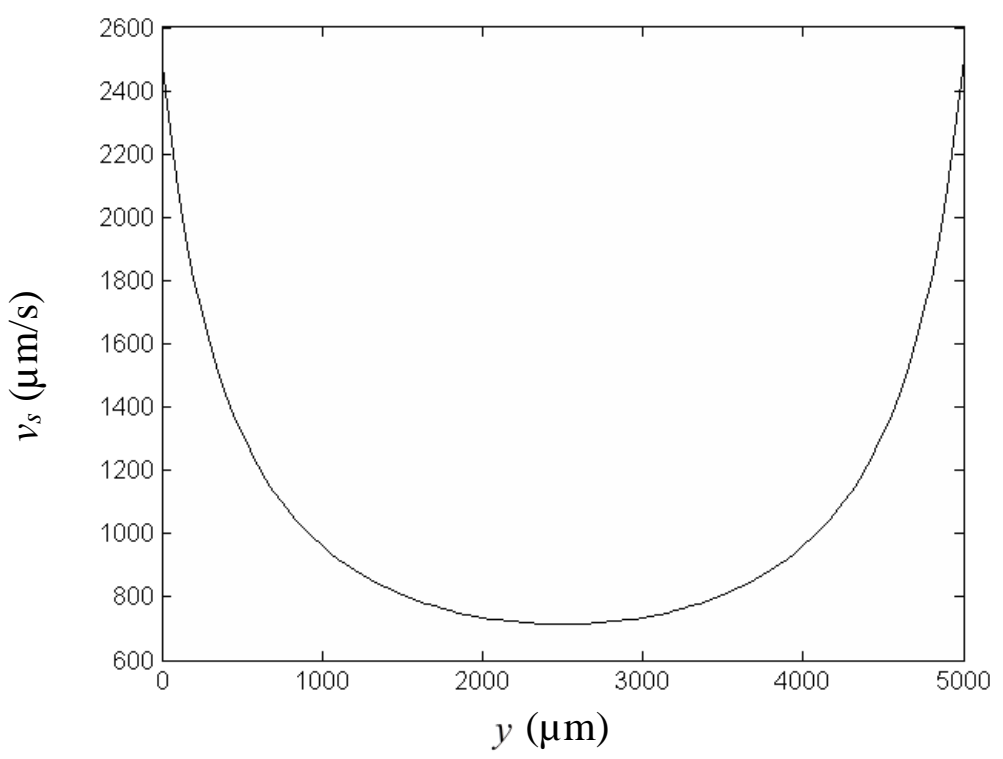

(b)

Figure 5-4 Desired, predicted, and measured channel centerline profiles for a desired parabolic depth of $z_{1}(y)=-490(y / 5000)^{2}+490 y / 5000+49 \mu \mathrm{m}$; (b) predicted velocity function required to machine the desired profile. A maskless source was used with an equivalent point source strength of $K=z_{c} v_{c}=123 \times 10^{3} \mu \mathrm{m}^{2} / \mathrm{s}$.

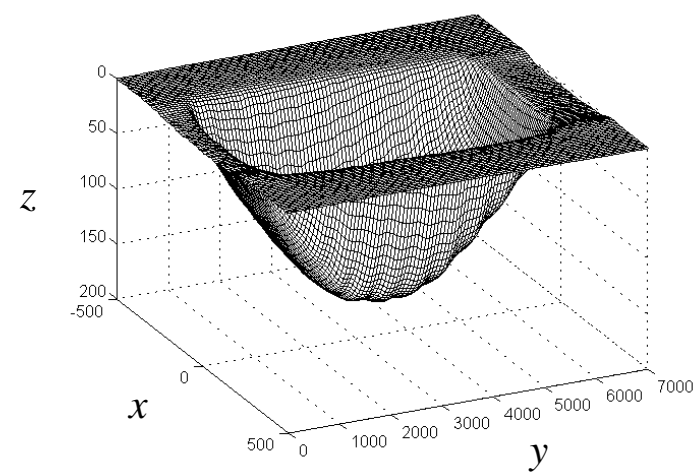

(a)

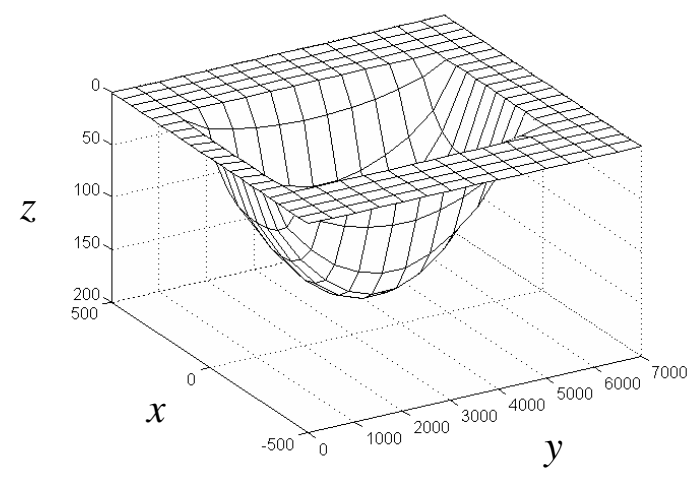

(b)

Figure 5-5 (a) Measured and (b) predicted 3D profile for channel of Figure 5-4. Dimensions in $\mu \mathrm{m}$. 


\subsubsection{Predicted and measured channels with depth varying sinusoidally with long wavelength}

Figures 5-6 and 5-7 show that the inverse method solution was able to accurately predict the velocity required to machine a micro-channel whose desired profile varies sinusoidally over 2 periods as $z_{1}(y)=140+100 \cos (4 \pi y / 5000) \mu \mathrm{m}$. The measured centerline profile is in excellent agreement in both amplitude and frequency with the originally desired one, with a maximum point by point average error in depth of less than $7.8 \%$. In other trials (not shown), the predictions were found to be equally good regardless of the overall length (i.e., number of periods the pattern repeated) of the channel. Figure 5-8 shows a scanning electron micrograph (SEM) image of a typical channel machined in this manner.

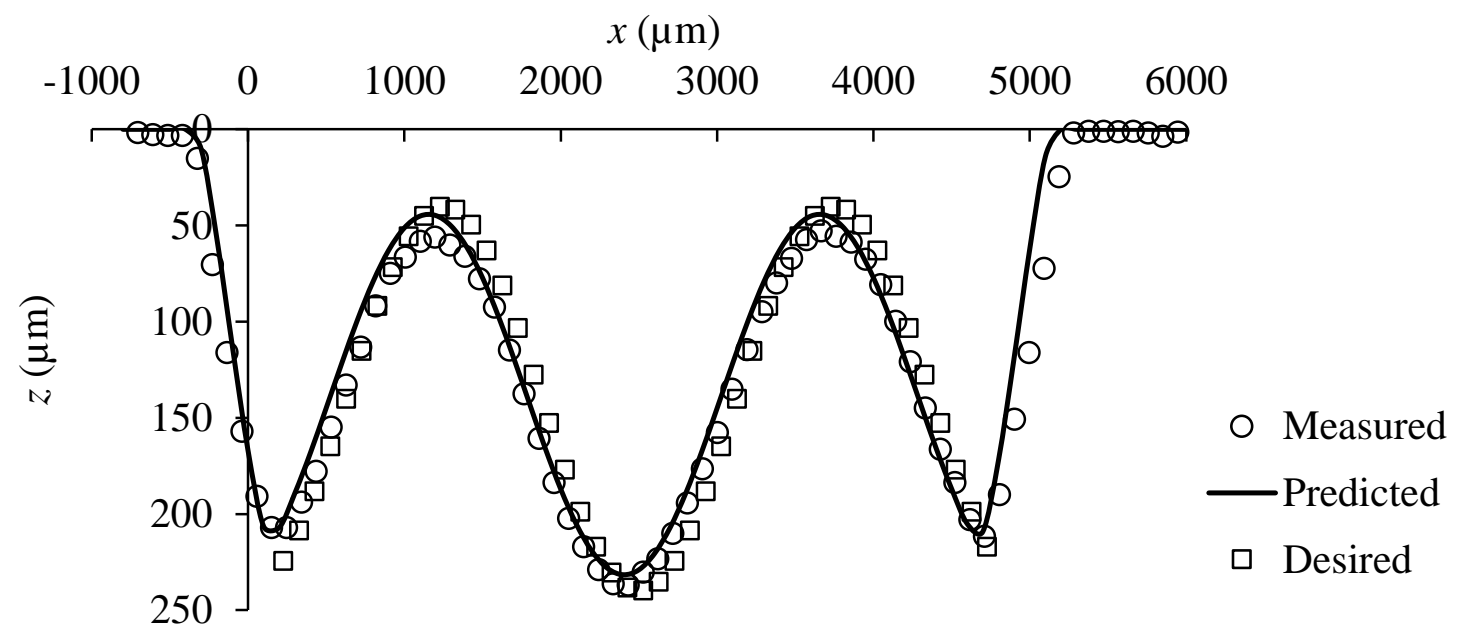

(a) 


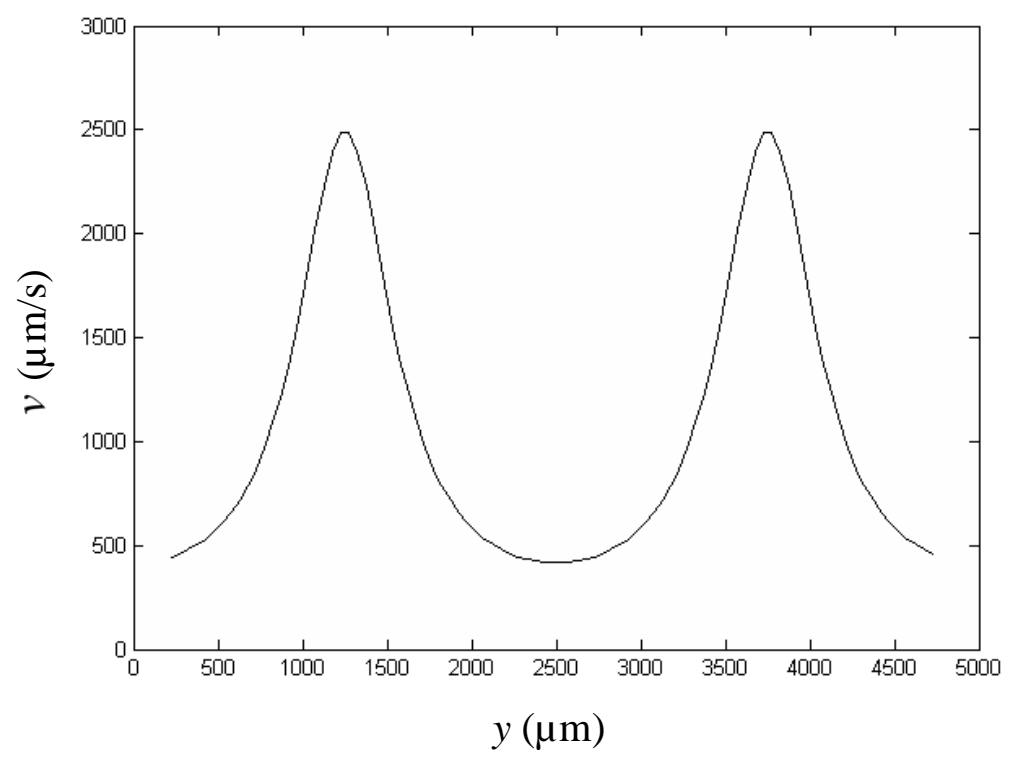

(b)

Figure 5-6 Desired, predicted, and measured channel centerline profiles for a desired sinusoidal depth of $z_{1}(y)=140+100 \cos (4 \pi y / 5000) \mu \mathrm{m}$; (b) predicted velocity function required to machine the desired profile. A maskless source was used with an equivalent point source strength of $K=z_{c} v_{c}=100 \times 10^{3} \mu^{2} / s$.

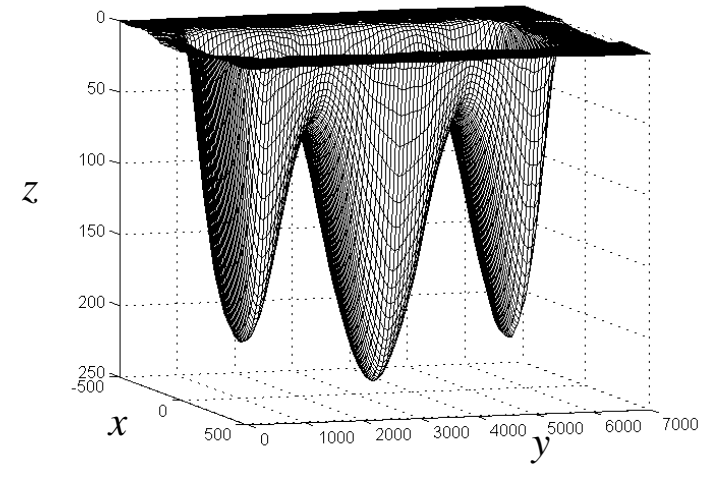

(a)

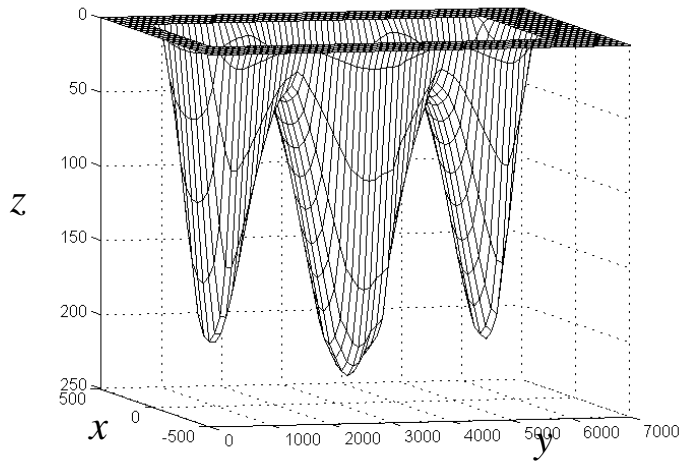

(b)

Figure 5-7 (a) Measured and (b) predicted 3D profile for channel of Figure 5-6. Dimensions in $\mu \mathrm{m}$. 


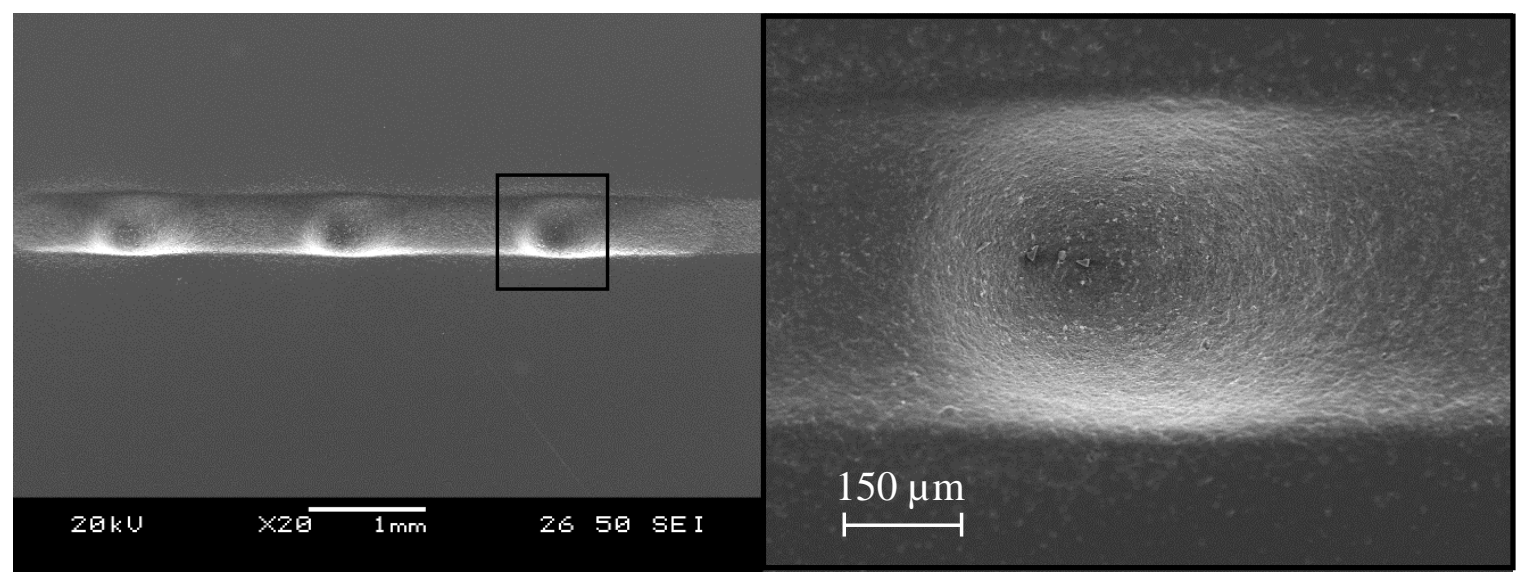

(a)

(b)

Figure 5-8 SEM image (a) of a channel machined using the maskless technique with depth varying sinusoidally for 3 periods over a $5 \mathrm{~mm}$ length; (b) shows a magnified view of the square region highlighted in (a).

\subsubsection{Conditions under which a point source solution will fail}

In Sections 5.3.2.1-5.3.2.3, it was demonstrated that the point source inverse solution could be used to accurately determine the velocity required to machine a variety of topographies having features (e.g. distance between two consecutive extremums) that were relatively large compared to the size of the source. It will now be shown that if the features are of similar size as the source, the point source solution cannot be used.

Figure 5-9 shows the predicted velocities corresponding to point and Gaussian solutions to the inverse problem for the machining of a channel having sinusoidal texture of $z_{1}(y)=75+35 \sin (6 \pi y / 5000) \mu \mathrm{m}$, i.e. with a shorter wavelength than in Figure 5-6. To machine the channel, the $460 \mu \mathrm{m}$ nozzle was used at a much higher $(10 \mathrm{~mm}$, rather than $100 \mu \mathrm{m})$ stand-off distance than previously used. At this larger standoff, a Gaussian erosive efficacy resulted with a footprint that was approximately $1.7 \mathrm{~mm}$ wide. In other words, the source footprint width and the peak-to-peak distance of the sinusoid were approximately the same. In order to be consistent with the defined normalizing factor of the minimum desired depth $z_{1 \text { min }}$ in Chapter 4 , this implies a Gaussian source with $\beta=0.11$. As Figures 5-9 and 5-10 show, the two predicted velocities, and thus the resulting machined profiles, deviate significantly from each other because changes in the desired profile depth occur over lengths comparable to the source width. As predicted in the Chapter 4, there is a significant error introduced by using the point source solution in this case, i.e. 
the predicted profile obtained using the Gaussian solution fits the desired profile much closer than that predicted using the point source solution. This contrasts with the cases considered in Section 5.3.2.3, where the changes in depth occurred over larger distances, and the Gaussian and point source solutions were found to work equally well.

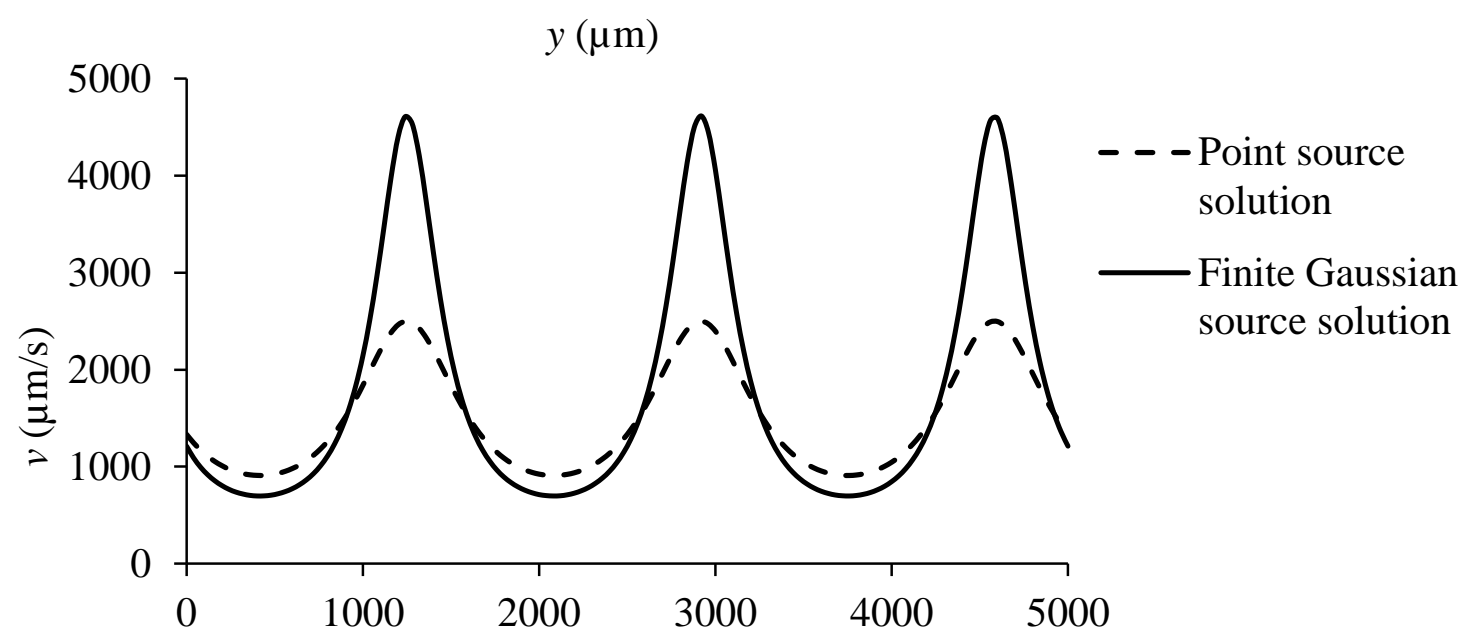

Figure 5-9 Velocity function predicted by finite Gaussian source solution and point source solution.

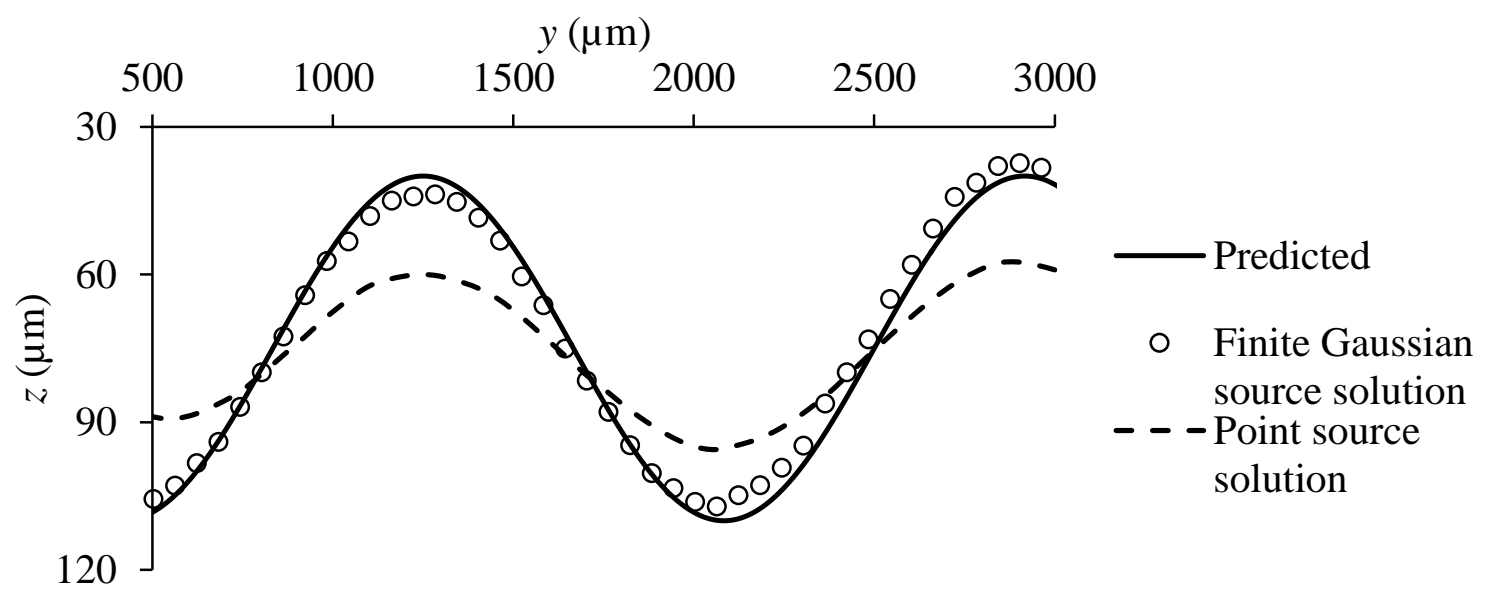

Figure 5-10 Measured profiles resulting from machining with velocity function predicted by finite Gaussian source solution and point source solution, compared to desired profile with wavelength of $5000 / 3 \mu \mathrm{m}$. The Gaussian footprint of the source was approximately $1.7 \mathrm{~mm}$ wide $(\beta=0.11)$. 


\subsubsection{Pockets with texture in two directions}

A pocket with a desired texture of $z(y)=85+55 \cos (6 \pi y / 5000)$ and $z(y)=85+55 \sin (6 \pi y / 5000)$ alternating every $\Delta x=500 \mu \mathrm{m}$ is shown in Figure 5-11(a). The predicted velocity functions in order to machine strips centered every $\Delta x$ are given in Figure 5-12. As Figure 5-11 shows, the desired and measured textures match closely, e.g., the profiles at any $x$ location were within $7.1 \%$ of each other.

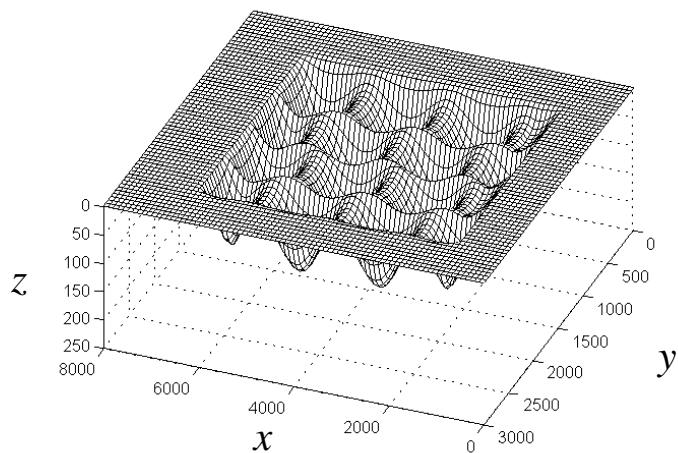

(a)

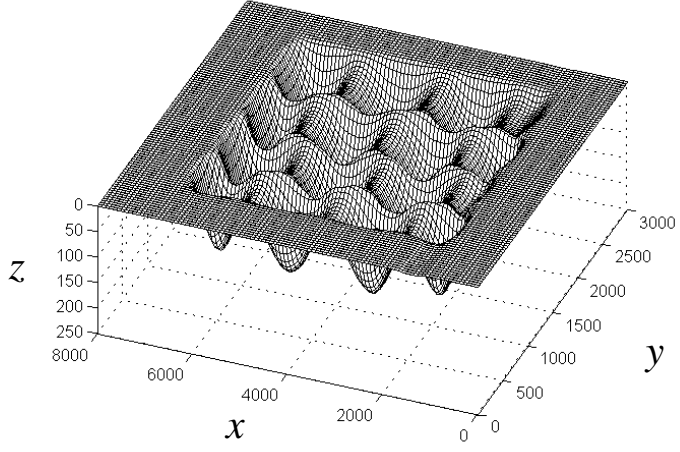

(b)

Figure 5-11 (a) Desired 3D pocket texture and (b) texture resulting from machining using the predicted velocities in Figure 5-12. A maskless source was used with an equivalent point source strength of $K=z_{c} v_{c}=75 \times 10^{3} \mu m^{2} / s$. Dimensions are in $\mu \mathrm{m}$.

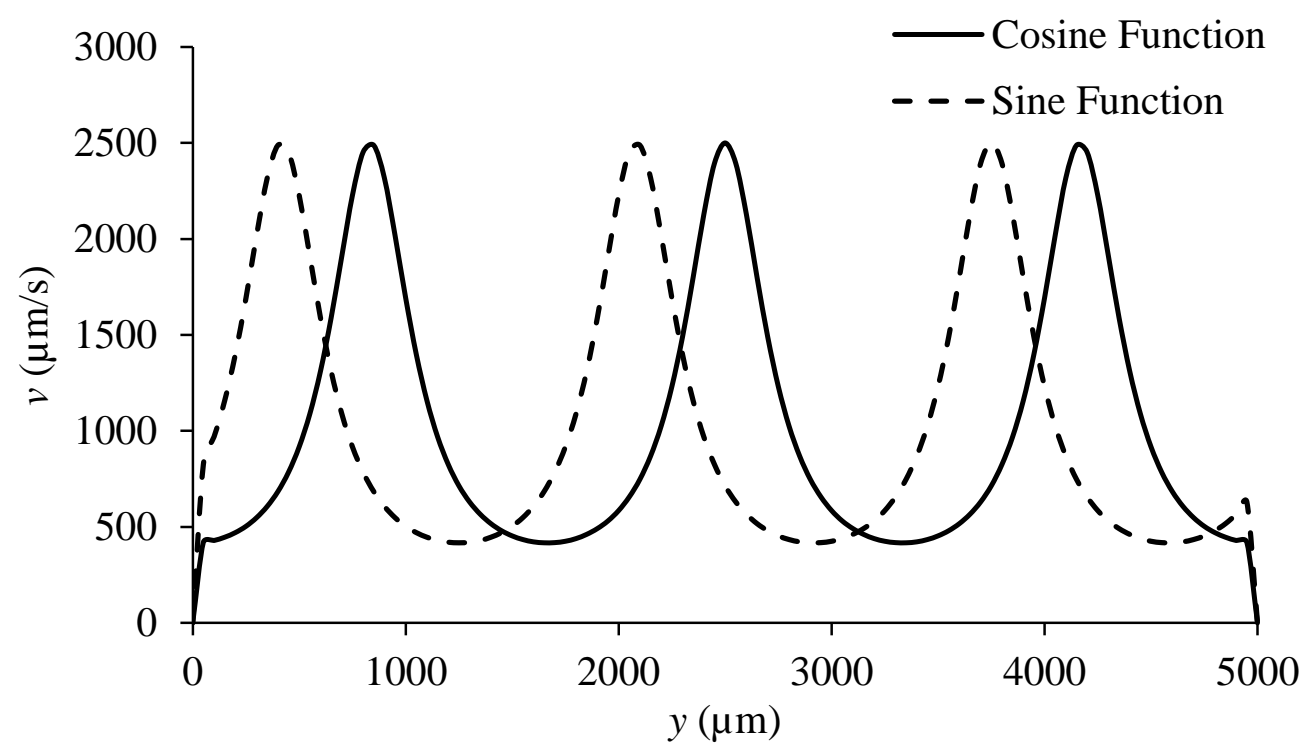

Figure 5-12 Velocity functions required to machine the desired 3D pocket texture in Figure 5-11 (a). 
The effect of varying the offset distance $\Delta x$ used between the desired alternating textures described above is investigated in Figure 5-13. Although the texture resulting from use of the velocities in Figure 5-12 at all offsets could still be predicted reasonably well, the use of $\Delta x<500$ $\mu \mathrm{m}$ resulted in textures that visibly differed from that desired (Figure 5-11(a)). This provides experimental verification for the minimum strip size recommendations presented in Section 4.2.4.2. An image of the machined pockets using $\Delta x=500 \mu \mathrm{m}$ (Figure 5-11(a)) and $\Delta x=400 \mu \mathrm{m}$ (Figure 5-13(b)) is shown in Figure 5-14.
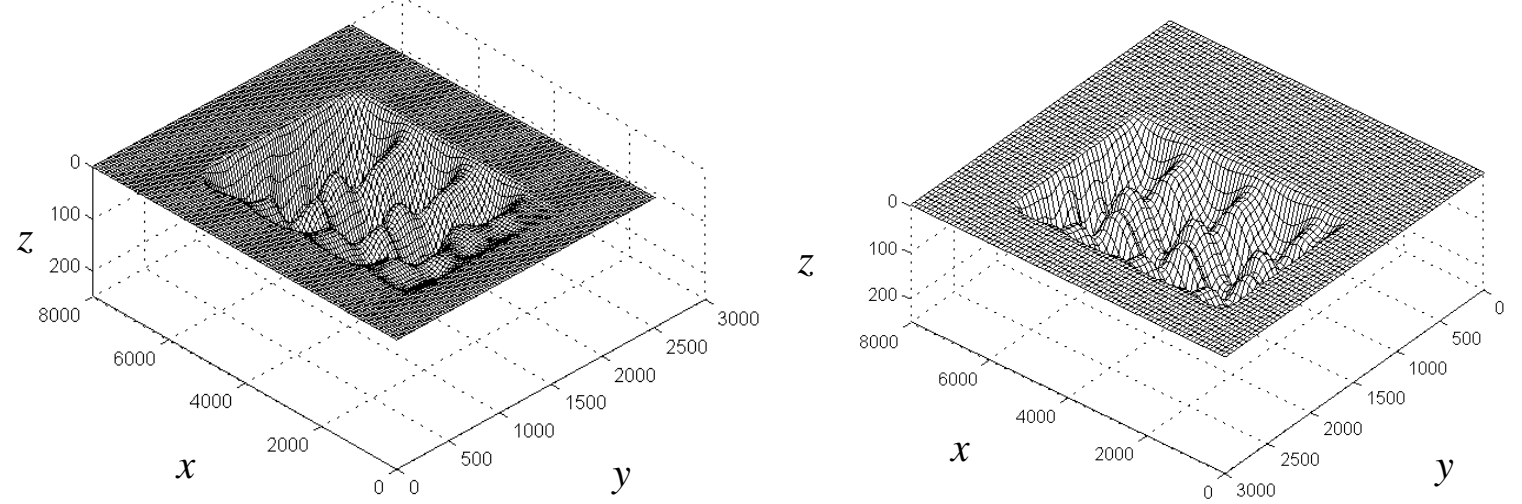

(a)
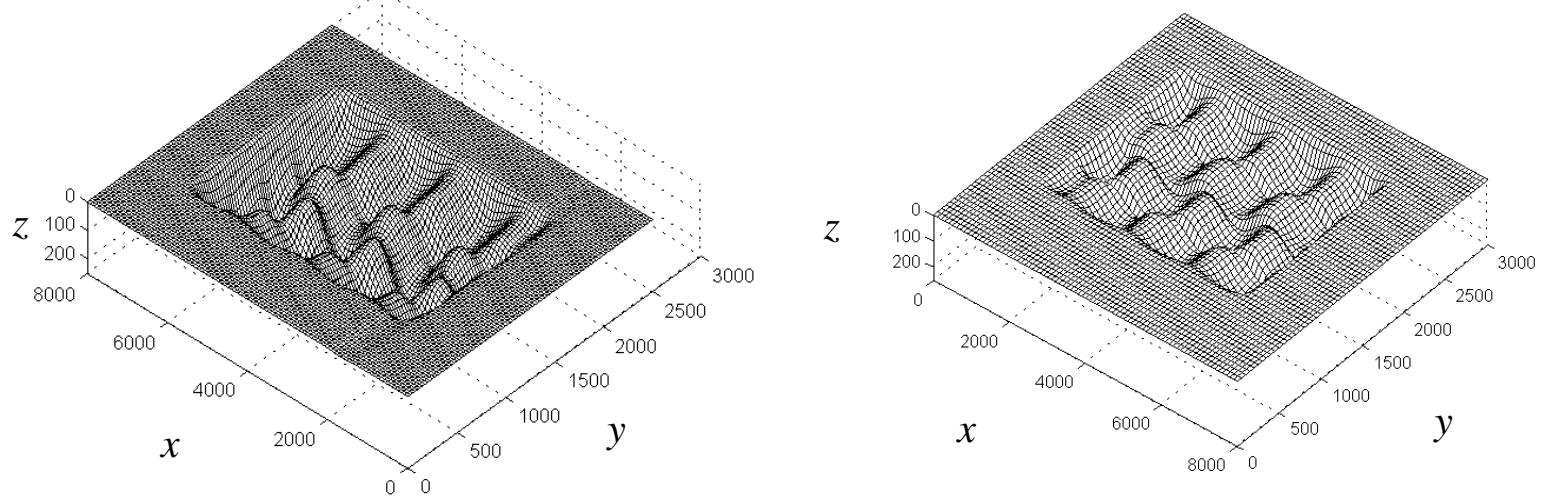

(b)

Figure 5-13 Predicted (right side) versus experimental (left side) textures for offset of (a) 300 and (b) $400 \mu \mathrm{m}$. As the offset becomes smaller, the pattern deviates from the desired one in Figure 5-11(a). Dimensions are in $\mu \mathrm{m}$. 


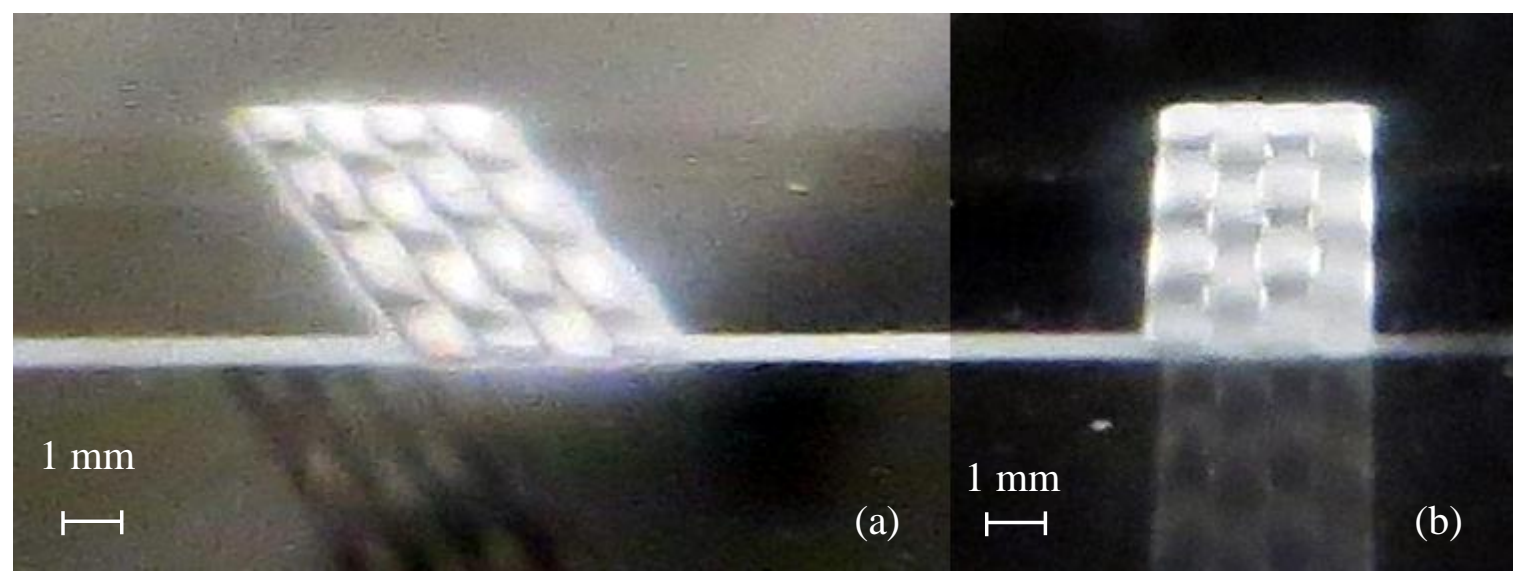

Figure 5-14 Images of textures machined using maskless technique with (a) $\Delta x=500 \mu \mathrm{m}$ and (b) $\Delta x=400 \mu \mathrm{m}$. The camera was tilted to capture the patterns.

\subsection{Summary}

Inverse methods presented in Chapter 4, which were used to predict the path and velocity of an AJM source, must take in order to sculpt a variety of prescribed three-dimensional topographies. The predictions were then used in machining experiments. Overall the following conclusions were reached:

(viii) The inverse methods of Chapter 4 were experimentally verified to be effective for both gradient-etching specified textures into channels and pockets, and for controlling the channel cross sectional shape, using AJM. Typically, the maximum error between the measured topographies on features machined using the predicted velocities, and the originally desired topographies was less than 7\%. The methodology is relatively simple because it is in the form of an analytical solution rather than a numerical algorithm.

(ix) When the source size was comparable to the distance between two consecutive extremums in the desired feature, inverse solutions assuming a Gaussian source were required to predict the correct source velocity. The error introduced by assuming a point source in all other cases is generally small, and can be predicted using the equation provided in Chapter 4. 
(x) When machining pockets with texture in two directions, a critical offset distance exists (0.75 times source width), below which the machined texture deviated from the desired one.

Chapter 6 will introduce a novel rotating mask apparatus that can be used to produce a much wider range of eroded topographies than is possible by simply changing the source velocity. An alternative optimization-based approach, to the inverse problem of determining the erosive efficacy to produce a desired topography will also be introduced. 


\section{Chapter 6 A rotating mask system for sculpting of three-dimensional features using abrasive jet micro-machining}

This chapter is based on the following submitted paper:

M. R. Sookhak Lari, A. Ghazavi, and M. Papini. "A rotating mask system for sculpting of threedimensional features using abrasive jet micro-machining" Journal of Materials Processing Technology, under review.

\subsection{Introduction}

Recently, there has been increased interest in applications for micro- channels with nonconventional shaped cross sections, especially in the microfluidics, where flow and heat transfer characteristics may depend on shape [2]. Di Carlo [90] presented a critical review on the effect of fluid inertia in micro-fluidic applications such as mixing, particle separation and bioparticle focusing, and discussed how secondary flow in curved channels of various cross-sectional shapes can affect the migration of particles. Theoretical investigations have revealed that the lift force acting on particles depends on their position within a micro-channel, which can depend on its cross sectional profile [91,92]. Sharma et al. [68] investigated performance of rectangular and trapezoidal (A and V-type) micro-channels with water and liquid gallium as coolants. They found that the cross sectional geometry is an important factor in temperature dissipation and required pump power. Akbari et al. [69] investigated an approximate model for pressure drop in a microchannel of arbitrary cross section, and found that cross-sectional perimeter was a suitable characteristic length scale to be used in the proposed model. Bahrami et al. [70] also presented an approximate model for the pressure drop of laminar flow in micro-channels of arbitrary crosssection.

As discussed in detail in Chapters 4 and 5, most microfabrication technologies capable of sculpting desired micro-channel shapes are limited in the range of shapes they can produce. These include high speed multiphoton absorption polymerization (MAP) [55], backside diffused-light exposure [56], $\mathrm{KOH}$-anisotropic etching technique [93] and gradient chemical etching [74]. Abrasive jet micromachining (AJM) uses jets of impacting abrasive particles to mechanically etch 
the target surface. Its directional etch capability makes it well suited for the sculpting of a wide variety of micro-features with three-dimensional (3D) topographies. It has been used to fabricate features such as micro-pumps in glass [47], monolithic millimeter high microstructures [58], accelerometers and millimeter size cantilever beams devices in glass [44].

Traditionally AJM has been performed by attaching a set of masks with a desired opening directly to a target surface. The machined feature size in this case simply depends on the size of the mask opening. Various types of metallic, polymeric, and elastomeric masks have been used. The metallic masks, usually stainless steel or copper, are patterned with openings and usually clamped to the target surface [58]. A notable exception is the work that was presented in Chapter 2, who introduced an adjustable metallic shadow clamp apparatus that could move with nozzle attached to it, making it possible to change the size of the erosive footprint for a given nozzle pass. Polydimethylsiloxane (PDMS) masks have been cast by creating a mold of polyester polyurethane or SU8 epoxy using photolithography $[40,94]$. Photosensitive resins have also directly been used as masks. For example, Liao et al. [95] investigated the effect of rebounding particles on the erosion of holes in a photosensitive resin mask during sand blasting, and presented a relationship between the desired hole size and required mask opening. Wensink et al. [29] tested polyimide, a negative liquid resist coating, as a mask material for powder blasting, but found that its high erosion rate made it unsuitable for deep powder blasting. Slikkerveer et al. [96] presented the erosion behavior of three photosensitive polyurethane acrylates (Ordyl BF410, LF55G1 and Ebecryl 270), and found a relation between their erosion resistance and the blasting impact angle.

Self-adhesive ultraviolet (UV) cured elastomeric films such as RapidMask (IKONICS Imaging, Duluth, MN, USA) have also recently been developed for use as masks in AJM applications. In this case, patterns are created on transparencies which are placed over the films and then exposed to UV light. The cured portion of the mask becomes brittle, and when attached to the target material and exposed to an abrasive jet, is eroded quickly to produce the patterned openings in the surrounding uncured, erosion resistant mask. Ghobeity et al. [33] showed that such masks can be used with AJM to reliably micro-machine features as small as $50 \mu \mathrm{m}$ in micro-fluidic chips. Achtsnick et al. [34] evaluated four types of photosensitive films concluding that, although their poor erosion resistance made them limited in terms of achievable feature depth, they were nonetheless easy to pattern and thus suitable for a complex designs. 
Regardless of mask material, in traditional AJM systems, the particle jet is scanned directly over the mask openings, so that the depth of the resulting feature is controlled by increasing the particle mass flux, the blasting pressure, or the number of nozzle scans. The overall shape of the resulting feature, however, is not controllable, i.e., the cross-sectional shape of a channel is always approximately $\mathrm{V}$-shaped for brittle materials, and U-shaped for ductile ones, with no control over, e.g., the channel sidewall slope [39]. Existing examples of the use of abrasive jet technology to control feature shape includes the work of Ghobeity et al. [76] for AJM, Billingham et al. [79] for abrasive water jets, and Tamannaee et al. [77] for abrasive slurry jets, who all used superposition of adjacent overlapping constant traverse speed passes to control the final shape of relatively shallow and wide machined features. Such adjacent pass approaches, however, are relatively time-consuming since they require many relatively rapid nozzle traverses in order to avoid complications associated with the dependence of the erosion rate on the slope of the kerf produced in a given pass [77].

In Chapters 4 and 5, a solution to the inverse problem of determining the non-uniform nozzle traverse velocity required to achieve any net desired 'erosive efficacy' [39] in an AJM operation was presented. In this context, the erosive efficacy, i.e., the sum of the superimposed incident particle flux and velocity distributions when moving the jet 'footprint' over the target surface, is directly proportional to the initial shallow shape of the machined surface. As it was demonstrated in Section 4.3, the methodology could be effectively used to create essentially any desired non-uniform depth texture along the length of micro-channels or in micro-pockets. Using an oscillation technique in Chapter 3, it was showed that it was possible to create a family of microchannels having various $\mathrm{W}$-shaped cross-sections. However, the range of feature shapes that can be made in this manner is limited due to the finite time required to accelerate and decelerate the nozzle as it moves from one point to another.

This chapter presents a novel rotating mask apparatus (RMA) that allows the instantaneous control of the AJM footprint size and shape so that any desired net erosive efficacy can be produced. 


\subsection{Experiments}

\subsubsection{Basic concept of the RMA}

In contrast to traditional AJM which uses a single mask with a continuous pattern on it, the RMA system uses a series of patterned elastomeric masks that are attached to a disk that rotates between a single source (nozzle) and the target material (Figure 6-1). The disk has openings along the circumference over which the patterned masks, consisting of series of holes placed in different configurations, are placed. The jet of abrasive particles is directed towards the disk, passing through the patterns in the masks such that the erosive efficacy reaching the target in a given rotation is the sum of the individual erosive efficacies passing through the holes in the mask. The holes can be thought of as the origins of small erosive efficacy sources; thus, by changing their radial positions in a pattern, a full rotation simulates the desired movement of a single source across the target surface. Since the radial position of the holes can be chosen discretely in a wide variety of combinations, simulation of a wide variety of movements of single source can be achieved, including near instantaneous movement from one location to a relatively distant one, which is not physically possible by, e.g., moving a nozzle. The size and number of holes at different radial locations controls the size and number of sources seen by the target in a given rotation, i.e. the relative size and magnitude of the net erosive efficacy reaching the target at a given $x$ (Figure 6-1). By appropriate pattern design (Section 6.3.3), virtually any erosive efficacy, and thus desired eroded topography, can be created. 


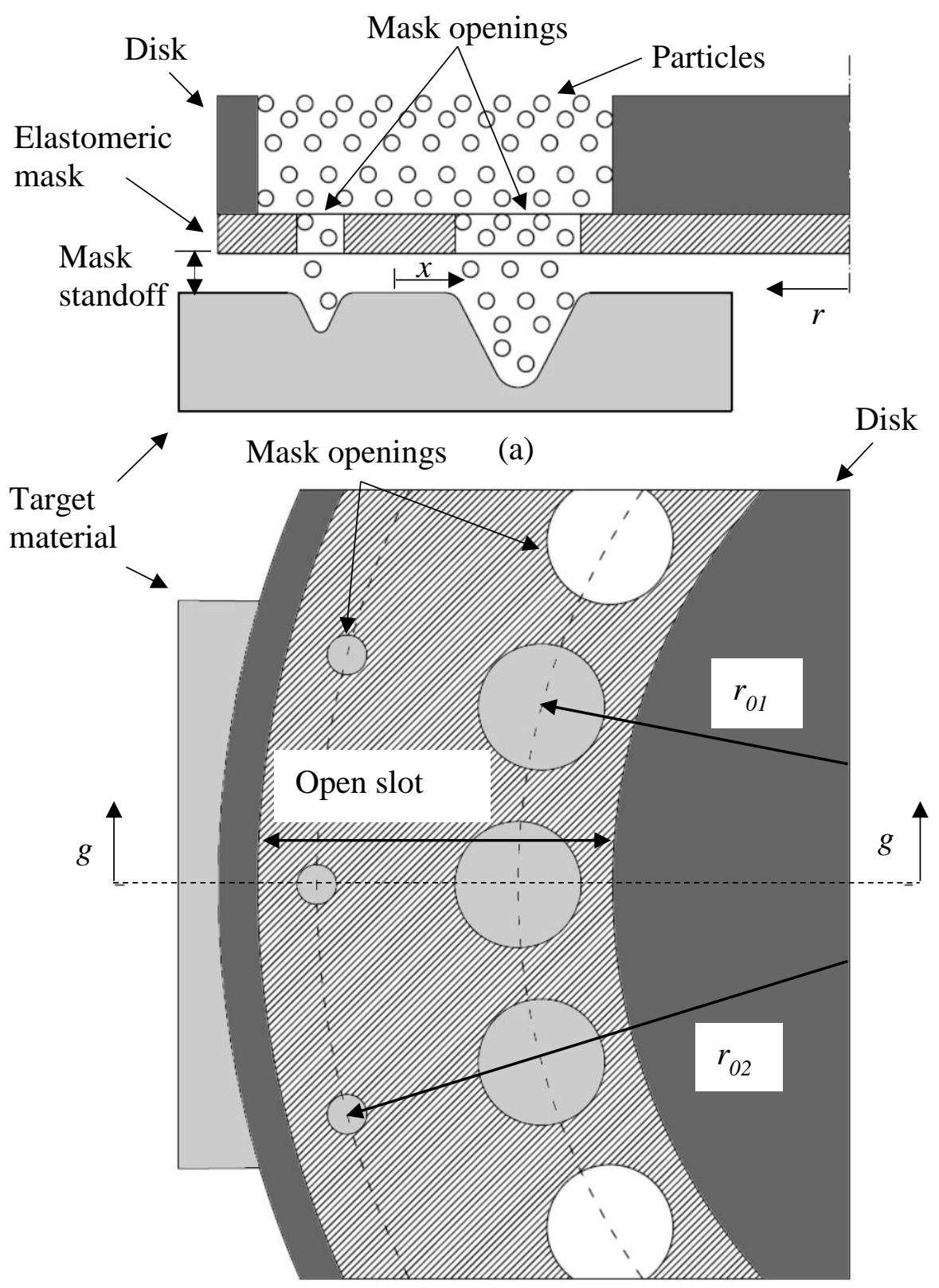

(b)

Figure 6-1 (a) Side (section g-g) and (b) top views of conceptual schematic of RMA disk, showing elastomeric mask suspended across disk slot. $r_{0 i}$ are the radial positions of base arcs on which holes were cured in the mask material. Crosshatched region is the open slot in the disk over which the patterns in the mask are suspended. Schematic is not to scale. 


\subsubsection{Detailed RMA Setup}

Figure 6-2 shows schematic of the RMA. An AccuFlo AF10 Micro-Abrasive Blaster (Comco, Inc. Burbank, CA, USA) created the jet consisting of a mixture of $200 \mathrm{kPa}$ pressurized air and aluminum oxide particles of $25 \mu \mathrm{m}$ nominal diameter that was made to pass through a 0.3 $\mathrm{mm}$ by $3.8 \mathrm{~mm}$ rectangular nozzle. More information on the blasting apparatus can be found in Chapter 2. The Borofloat glass (Swift glass Co. Inc., Elmira, NY, USA) target samples were moved below the fixed nozzle and RMA by two computer controlled, perpendicular stages (Aerotech Inc., Pittsburgh, PA, USA) with positioning resolutions of $0.5 \mu \mathrm{m}$.

A gearhead electric motor (RobotShop Inc. Mirabel, Quebec, Canada) with built in encoder was used to rotate a $1 \mathrm{~mm}$ thick and $50 \mathrm{~mm}$ diameter 6061 aluminum alloy disk which had four $24 \mathrm{~mm}$ long by $3 \mathrm{~mm}$ wide circumferential slots machined into it. The center-arc of the slots were $20.5 \mathrm{~mm}$ away from the axis of the shaft. The elastomeric mask (Section 6.2.3) was attached to the bottom of the disk, with the patterned portion aligned with the slots. To prevent wobble of the disk, the $1 \mathrm{~cm}$ steel shaft on which the disk was mounted passed through two aligned precision bearings. The motor was connected to a PID controller which allowed rotational speed control using a personal computer via a serial port. The apparatus was attached to a linear manual stage which allowed the mask standoff to be adjusted to be $\sim 300+/-10 \mu \mathrm{m}$ from the $3 \mathrm{~mm}$ thick glass target surface (Borofloat, Swift glass Co. Inc., Elmira, NY, USA). The distance between the nozzle exit and the top of the mask was held constant at $10 \mathrm{~mm}$. 


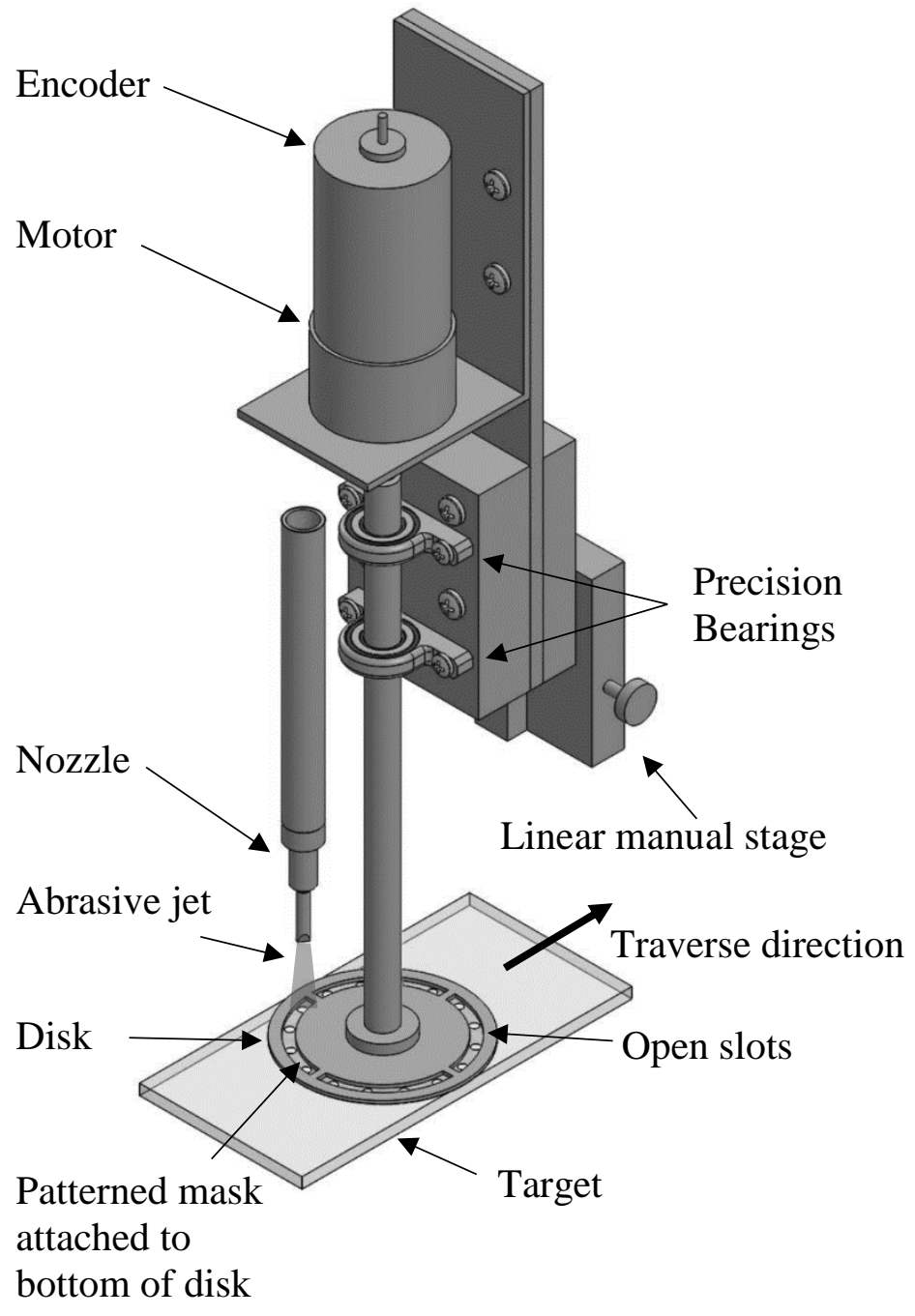

Figure 6-2 Schematic of rotating disk mechanism.

\subsubsection{Circular mask fabrication}

The circular masks were made from $100 \mu$ m thick High Tack (HT) Rapid Mask (IKONICS Imaging, Duluth, MN, USA), a flexible self-adhesive ultraviolet curing elastomeric mask material. Each master mask included two concentric guide circles for alignment purposes. The first one was used to define the boundaries of the disk on the cured masks, and coincided with the outer circumference of the disk (Figure 6-3, diameter=50 mm). The second was at a radial offset of 9 $\mathrm{mm}$ (Figure 6-3, diameter $=32 \mathrm{~mm}$ ) and was used to align the mask with an identical circle inscribed on the disk. The base arcs upon which patterns of holes on the mask were placed were in the region between the two circles, aligned with the position of the slots on the disk. For 
example, two base arcs were placed $19.95 \mathrm{~mm}$ and $21.05 \mathrm{~mm}$ away from the centre, allowing for $0.4 \mathrm{~mm}$ clearance from each side of the slot in the cylinder. Four U-shaped patterns (Figure 6-3 (a)) were also put on the mask in order to aid in aligning the mask and the opening slots circumferentially. To improve the alignment accuracy, a USB-microscope was used during the attachment process, such that the total radial wobble of the base arcs was measured with the USB microscope to be $<50 \mu \mathrm{m}$.

The area surrounding the patterns were hatched solid black. The number of base arcs on which the patterns were positioned (Figure 6-3 (a)), and the radius of the individual holes directly influenced the accuracy and shape of the resulting erosive efficacy (directly proportional to the shallow feature shape). By increasing the number of holes or the number of base arcs, the final cross-sectional geometry of the eroded shallow feature more closely resembled the desired feature. The minimum machinable feature size and its resolution is largely controlled by the size of the individual printed holes making up the pattern. In the present work, the holes were between 200 $\mu \mathrm{m}$ and $600 \mu \mathrm{m}$ in diameter, suitable for creating footprints that were $<1 \mathrm{~mm}$ wide. However, in principal, much smaller footprints might be made if high definition (HD) Rapid Mask were used instead of the currently used high tack (HT), since holes as small as $50 \mu \mathrm{m}$ can be made using HT [33]. An example of a final hatched pattern is depicted in Figure 6-3 (b).

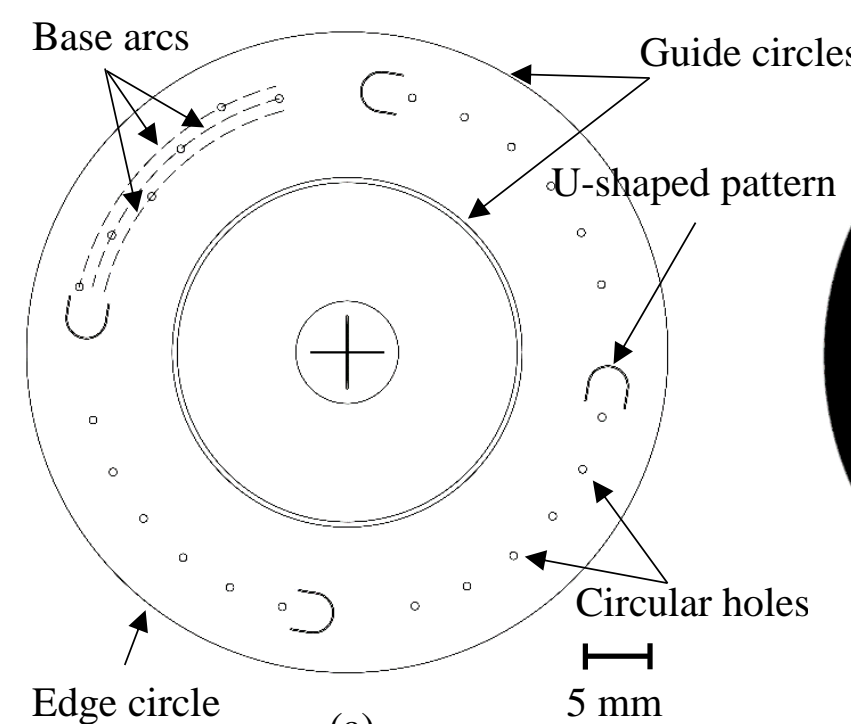

(a)

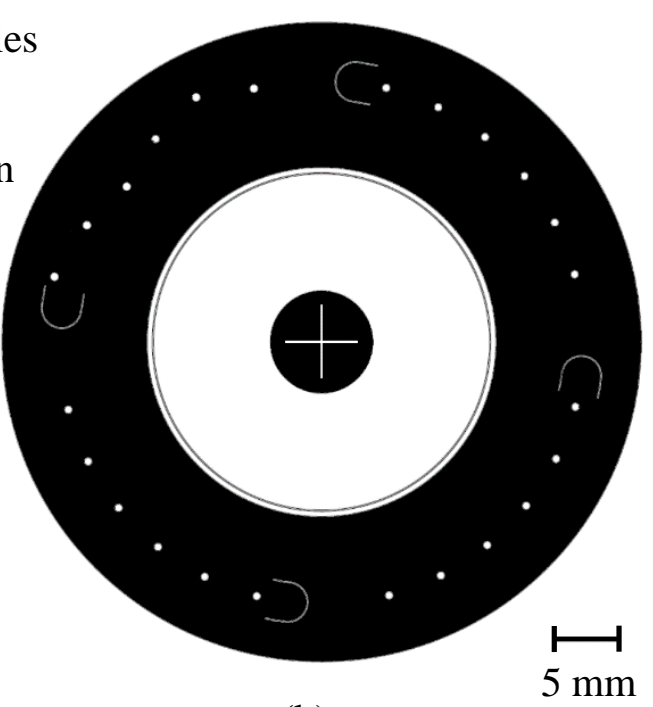

(b)

Figure 6-3 (a) Schematic of a mask showing the base arcs and alignment features, and (b) The mask master printed on the transparency 
The appropriate pattern needed to generate a desired erosive efficacy was determined using the methods presented in Section 6.3, and then created in AutoCAD (Autodesk Inc. San Rafael, California, U.S.). The visible layer which included the hatches was laser printed with a resolution of 25400 dpi (CAD/Art Services Inc. Oberman Lane Bandon, Oregon, U.S.) on transparency film. The high tack Rapid Mask was cut in a $55 \mathrm{~mm}$ by $55 \mathrm{~mm}$ square, laid over the printed pattern on the transparency film, and secured by taping its sides. A cylindrical UV curing unit (Letralite, IKONICS Imaging, Duluth, MN, USA) was used to subject the Rapid Mask with the attached patterned transparency to 2 minutes of UV radiation using the protocols suggested by the mask manufacturer [97]. In so doing, the Rapid Mask below the unhatched areas on the printed master transparency was exposed to UV light and cured. The UV exposure resulted in a pattern of cured (easily eroded brittle material) circles surrounded by uncured (difficult to erode elastomeric) mask. The abrasive jet was then run without the target below the disk, and the motor was spun at 1000 rpm for $\sim 6$ minutes. After that, the circles were 'open', i.e. the brittle cured portion was removed leaving holes in the mask suitable for use in the machining experiments.

\subsubsection{Mask erosion measurements}

Since the RMA apparatus utilized a non-traditional configuration in which patterned masks were suspended across open slots in the disk, rather than attached to a surface, it was of interest to measure their erosion resistance. This was investigated for three different configurations, each with three repeat trials, using the same nozzle, pressure, particles, and nozzle to surface standoff as described in Section 6.2.1:

1. Traditional configuration: A mask with a single $300 \mu \mathrm{m}$ circle on it was prepared and directly attached to a glass target. The mask was preconditioned to 'open the circle', i.e., exposed to the abrasive jet for $7 \mathrm{~s}$, sufficient to erode the cured brittle Rapid Mask within the circle, resulting in a hole. The hole in the mask was subjected to the abrasive jet for $1 \mathrm{~s}$ increments, and then visually examined with a light source underneath in order to determine whether the shape and size of the hole changed. The durability of the mask was considered as the time required for a $\sim 20 \%$ increase in hole diameter. 
2. Suspended stationary mask: A mask was attached to the bottom of the rotary disk setup as in Figure 6-1(b), but with only a single $300 \mu \mathrm{m}$ circle in a single base arc. Similar to (1), the mask was preconditioned by blasting for $13 \mathrm{~s}$ with the disk stationary, in order to create the hole. With the hole lined up with the jet and the disk not rotating, a channel was machined by moving the glass target at a constant scan speed of $0.1 \mathrm{~mm} / \mathrm{s}$ until the mask was completely worn out ( $60 \mathrm{~s})$. The machined channel centerline profile was measured along its length using an optical profilometer (NANOVEA ST400 Micro Photonics Inc., Irvine, CA, USA), and the point at which the mask failed was identified as an abrupt change in channel depth, which correlates with channel width due to the so-called blast lag effect that sees deeper channels produced by wider mask openings [98]. The durability was calculated by dividing the measured scan length to failure by the scan speed.

3. Suspended rotating mask: A mask with a pattern of 24 circles, each with a $300 \mu \mathrm{m}$ diameter, and each separated by $4 \mathrm{~mm}$, was attached to the bottom surface of the rotary disk. The mask was preconditioned by blasting while the disk spun at $1000 \mathrm{rpm}$ for $6 \mathrm{~min}$, resulting in a pattern of holes in the mask. The RMA was then used at $1000 \mathrm{rpm}$ to machine a channel into a glass target by moving it at a scan speed of $0.025 \mathrm{~mm} / \mathrm{s}$. Since the change in the channel depth for this case was more gradual compared to 2), failure of the mask was defined as the point at which the channel became $20 \%$ deeper than original due to erosion of the mask. Similar to 2), the time until failure of the mask was determined by dividing the length of channel until failure by the scan speed

\subsubsection{Effect of mask standoff on pattern transfer}

The ability of the suspended mask to transfer a pattern was examined for mask to surface standoff distances (Figure 6-1) between $300 \mu \mathrm{m}$ and $1.5 \mathrm{~mm}$. A line of circles with diameters between 100 and $600 \mu \mathrm{m}$, each with a 'bridge length' (closest distance between edges of two adjacent holes) of $800 \mu \mathrm{m}$ were cured on a piece of Rapid Mask. As shown in Figure 6-4, the cured mask was placed between a slotted holder plate and two feeler gauges whose thickness controlled the mask to target standoff distance. The construct was fixed together using hot glue, and mounted on a glass target which moved at a scan speed of $30 \mathrm{~mm} / \mathrm{s}$ below a stationary nozzle blasting 
abrasive particles. This very high scan speed ensured that the resulting features were less than $\sim 10$ $\mu \mathrm{m}$, thus eliminating under-etching that would serve to widen them at higher particle doses. Each experiment at a given mask standoff was repeated 3 times. The ability of the mask to transfer the pattern was assessed as the percentage difference between the radii of the holes in the mask and the holes eroded in the glass target, as measured using an optical profilometer.

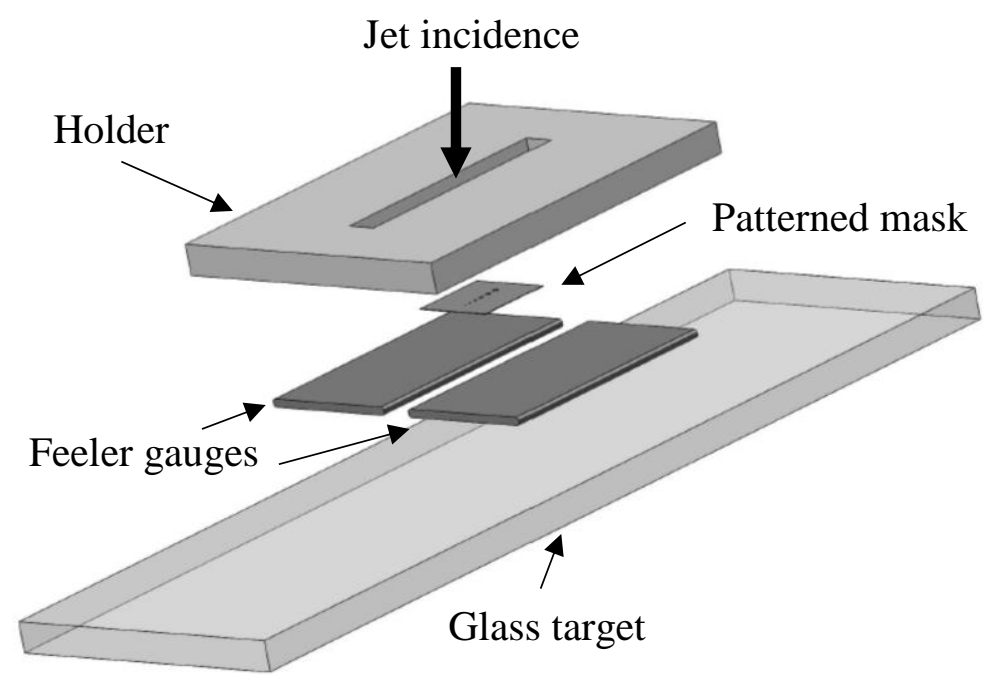

Figure 6-4 Fixture used for mask standoff experiments.

\subsubsection{Effect of distance between circles on mask}

Preliminary experiments showed that if the holes in the mask were too close together, an abrupt tear between them occurred while machining. It was of interest to determine the minimum allowable distance between holes in order to maximize the number of holes and base arcs, and thus improve machined feature resolution. A series of experiments was conducted where the bridge length $d_{c}$, (distance between the edges of the holes as shown in Figure 6-5) for both $300 \mu \mathrm{m}$ and $600 \mu \mathrm{m}$ holes was varied in the range $300 \mu \mathrm{m}-1300 \mu \mathrm{m}$ in $100 \mu \mathrm{m}$ increments. The mask was attached to the RMA and preconditioned as explained in Section 6.2.3 in order to open the holes in the pattern. The RMA was then run at $1000 \mathrm{rpm}$ while exposed to the abrasive jet (mass flow rate $=2.4-4.8 \mathrm{~g} / \mathrm{min})$ in intervals of $20 \mathrm{~s}(+/-1 \mathrm{~s})$. After each $20 \mathrm{~s}$ interval, the mask was inspected to determine whether abrupt mask tearing between holes had occurred. In this manner, the blasting time to tearing was determined, which could then be converted to particle dose by multiplying by the particle mass flow rate. 
The size of the nozzle footprint also affected the minimum practical distance between holes. The radial size of the footprint, $d_{m}$, of the jet on the disk largely determined the maximum usable radial distance between base arcs, $d_{r}$ (Figure 6-5). A larger footprint in the radial direction was desirable to minimize differences in particle flux between adjacent base arcs, and to facilitate alignment of nozzle with the mask. However, a small circumferential footprint, $d_{o}$, was desirable in order to localize the particle flux to the intended holes directly below the nozzle, i.e. to limit the amount of particles passing through holes further along the arc. For base arc diameters of $\sim 40$ $\mathrm{mm}$, a $0.3 \mathrm{~mm}$ by $3.8 \mathrm{~mm}$ rectangular nozzle at a $10 \mathrm{~mm}$ standoff oriented with the $3.8 \mathrm{~mm}$ dimension radially was found to be adequate.

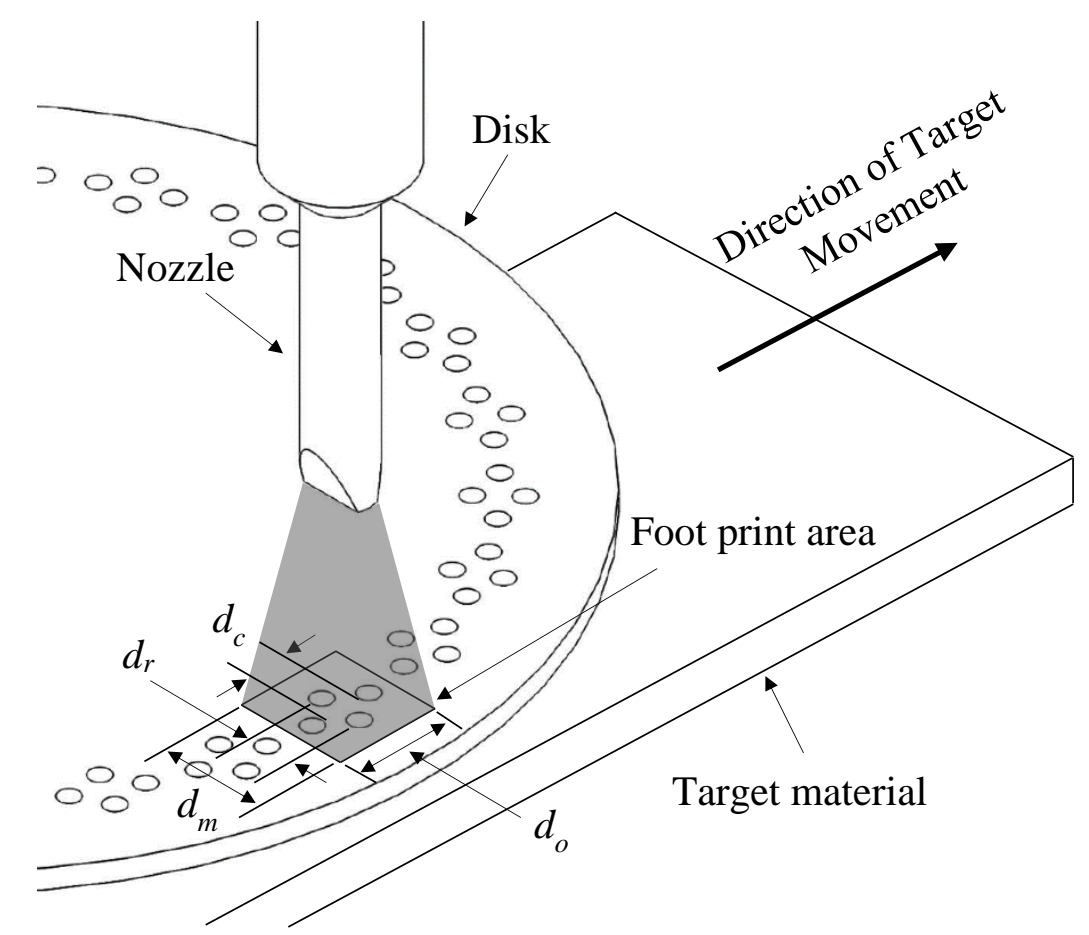

Figure 6-5 Schematic of hole placement on the disk and foot print of nozzle. Figure is not to scale.

\subsubsection{Effect of disk rotational speed}

The minimum $\omega_{\min }(\mathrm{rpm})$ and maximum $\omega_{\max }(\mathrm{rpm})$ rotational speeds required to ensure that the resulting channel was free of undue waviness, yet provided enough time (Figure 6-6) for particles to pass through the mask in a given rotation, were determined as 


$$
\begin{gathered}
\omega_{\min }=\frac{60 v}{d} \\
\omega_{\max }=\frac{30 v_{p} d}{\pi r\left(t_{m}+d_{p}\right)}
\end{gathered}
$$

where $v, d, t_{m}, d_{p}, r$ and $v_{p}$ are the target scan speed, mask hole diameter, mask thickness, nominal particle diameter, average base arc radius and average particle velocity, respectively. For typical conditions used in this chapter, i.e., $v=0.05 \mathrm{~mm} / \mathrm{s}, d=300 \mu \mathrm{m}, t_{m}=100 \mu \mathrm{m}, d_{p}=25 \mu \mathrm{m}, r=20.5 \mathrm{~mm}$ and $v_{p}=130 \mathrm{~m} / \mathrm{s}, \omega_{\min } \sim 10 \mathrm{rpm}$ and $\omega_{\max } \sim 145000 \mathrm{rpm}$. All experiments were performed at angular velocities between these values. For example, at $1000 \mathrm{rpm}$ and $0.1 \mathrm{~mm} / \mathrm{s}$ scan speed, the maximum waviness of a machined channel with depth of $100 \mu \mathrm{m}$ was found to be $<0.5 \mu \mathrm{m}$ which was similar to waviness of a nonrotating mask.

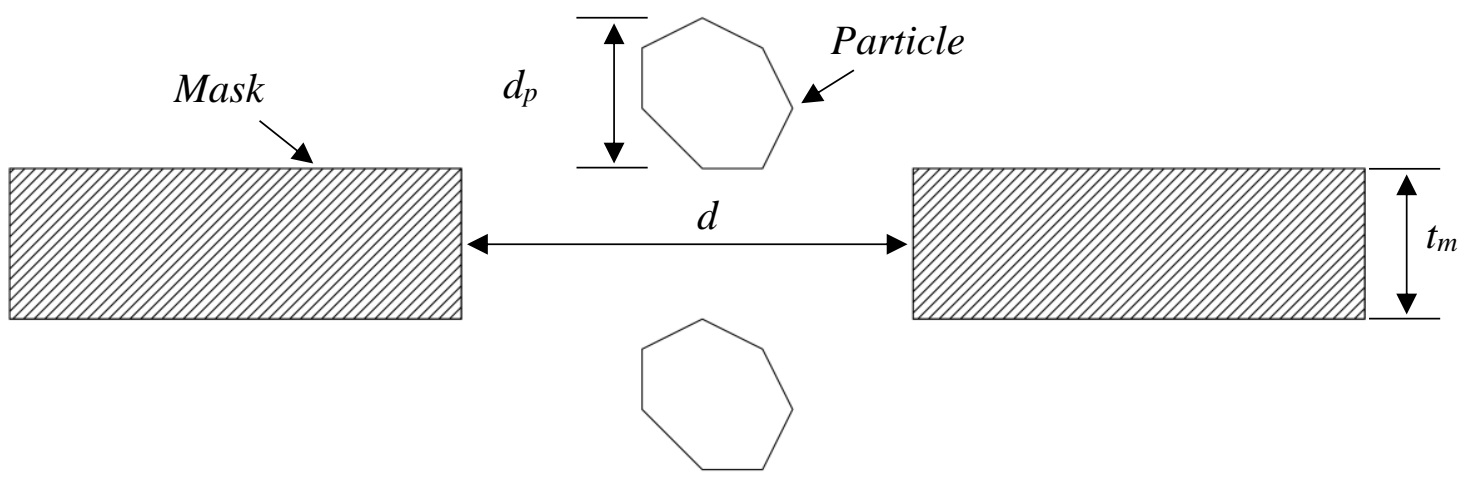

Figure 6-6 Schematic of a particle passing through a hole in the mask.

\subsubsection{Machining of channels of various cross-sectional shapes using the RMA}

The sidewall slope of channels machined using traditional AJM depends only on the material properties and size of the source erosive efficacy footprint. Thus for a given material and source size, the slope cannot be controlled unless the complicated machining procedure that Chapters 4 and 5 suggested is followed. One of the advantages of the presented apparatus is that it 
makes it possible to change the wall slope to desired values by changing the diameter of the printed holes. To demonstrate control of micro-channel sidewall slope, channels were machined using RMA masks with 24 holes on a $20.5 \mathrm{~mm}$ diameter base arc in three different patterns. The first used $300 \mu \mathrm{m}$ diameter holes, the second $600 \mu \mathrm{m}$ diameter holes, and the third with an equal number of alternating 300 and $600 \mu \mathrm{m}$ holes. The scan speed for all channels was kept constant at 0.1 $\mathrm{mm} / \mathrm{s}$.

Shallow W-shape, trapezoidal and wedge channels of various shapes were also machined in glass in order to illustrate the ability of the RMA to control the footprint shape. In all cases but one (Section 6.4.4.7 where $200 \mu \mathrm{m}$ holes were used), patterns of $300 \mu \mathrm{m}$ holes placed on different base arcs were used. The number of base arcs was inferred from the model of section 6.3.2 so that W-shaped, trapezoidal and wedge shaped footprints required 2, 5 and 5 base arcs, respectively. The number of required holes on each base arc for each desired footprint shape was determined using the optimization method of Section 6.3.3. The masks were all initially preconditioned for 6 minutes while the disk was rotating to open the circular holes in the cured mask material. Then, in order to avoid transients in particle abrasive mass flow rate and disk rotational speed, a protector shield was placed between the nozzle and the disk for $20 \mathrm{~s}$ before beginning to scan with a speed of $0.05 \mathrm{~mm} / \mathrm{s}$, an abrasive mass flow rate between $4 \mathrm{~g} / \mathrm{min}-6 \mathrm{~g} / \mathrm{min}$, and a rotational speed of $1000 \mathrm{rpm}$.

In all cases, the resulting machined channel profiles were scanned with the optical profilometer at a resolution of $25 \mathrm{~nm}$. Five cross sections were scanned along each channel. Each extracted profile was processed using a Matlab (The Mathworks, Inc.) code following the guidelines of Chapter 2 to determine its width. Each experiment was repeated three times and reported profiles were the ones closest to the average.

\subsection{Modelling}

\subsubsection{Functional form of the erosive efficacy through a series of holes in a given base arc}

Since the channels were all shallow, the total erosive efficacy footprint delivered to the surface, due to all particles that passed through the holes as the disk rotated, can be considered as directly proportional to the channel depth. In other words, $E^{*}=z^{*}$ where $E^{*}=E / E_{\max }$ and $z^{*}=z / z_{\max }$ are the normalized erosive efficacy and shallow channel depth, respectively $[77,79]$. 
Figure 6-7 shows an example of the measured footprint for $24,300 \mu \mathrm{m}$ holes on a $41 \mathrm{~mm}$ diameter base arc at $\omega=1000 \mathrm{rpm}$ and $\nu=0.1 \mathrm{~mm} / \mathrm{s}$.

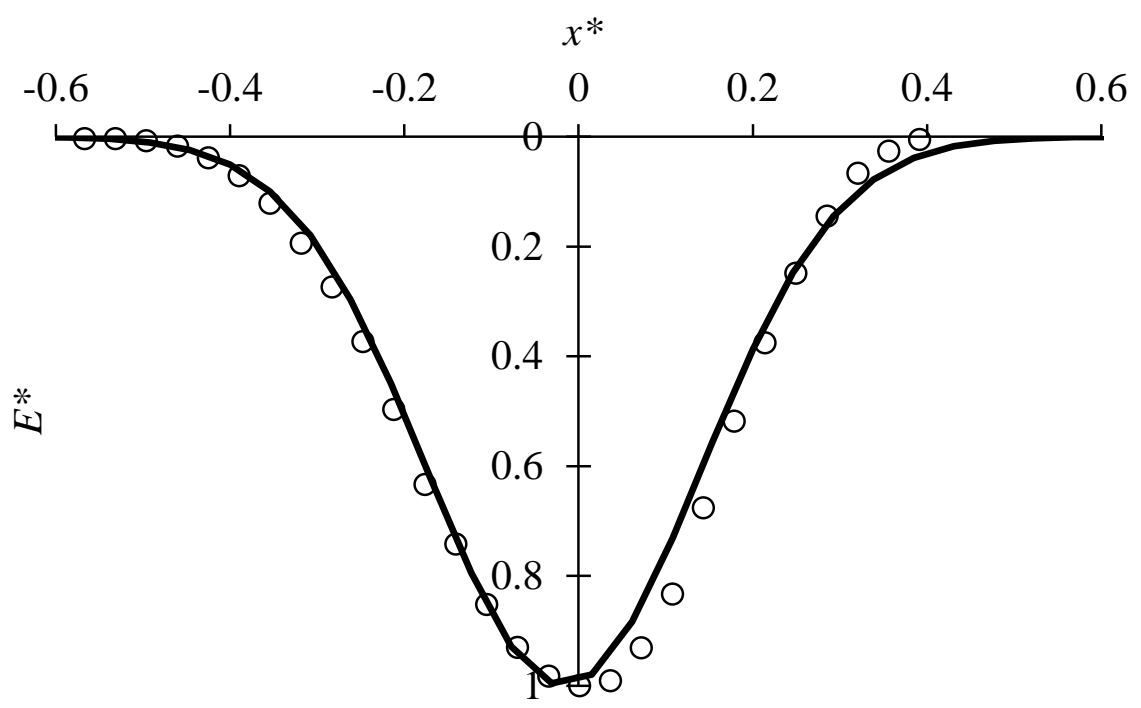

Figure 6-7 A non-dimensionalized channel $\left(x^{*}=x / 575\right.$ and $\left.E^{*}=z^{*}=z / 112\right)$ machined with RMA and a $300 \mu \mathrm{m}$ hole pattern with single base arc (circles). Solid line shows the best fit curve of Eq.(6.3) with $\beta=4.5$.

Similar to that measured for circular nozzles, and through contact and shadow masks [39], Figure 6-7 shows an approximately Gaussian non-dimensional net erosive efficacy $\left(E^{*}\right)$ footprint, i.e.:

$$
E^{*}\left(x^{*}\right)=e^{-\beta^{2} x^{* 2}}
$$

where $\beta$ describes the footprint size, and $x^{*}=x / w$ where $w$ is the width of the channel.

\subsubsection{Net erosive efficacy through a pattern of holes on different base arcs}

A given $x$-location on the surface is exposed to an erosive efficacy each time a hole above it passes through the abrasive jet. The greater the number of holes at that location, the greater the erosive efficacy. If holes on adjacent base arcs partially overlap, then a surface may see erosive efficacy from multiple base circles. The total normalized erosive efficacy $E_{T} *$ to a given location 
on the surface is thus simply the superposition of the erosive efficacies passing through the holes above the surface at that location:

$$
E_{T}^{*}\left(i, j, d_{i, j}^{*}, \beta_{i, j}, x^{*}, x_{0 i}^{*}\right)=\sum_{i=1}^{i_{\max }} \sum_{j=1}^{j_{\max }} d_{i, j}^{*} e^{-\beta_{i, j}{ }^{2}\left(x^{*}+x_{0 i}^{*}\right)^{2}}
$$

where $i$ is the base arc number, $j$ is the hole number on a given base arc (Figure 6-8 (b)), $d_{i, j} *$ is normalized depth (i.e., depth of one hole divided by the maximum depth of the resulting superposition). The values of $i_{\max }$ and $j_{\max }$ represent the total number of base arcs and holes on a given base arc, respectively. As shown in Figure 6-8 (a), $x^{*}$ and ${ }_{x_{\mathbf{O} i}^{*}}^{*}$ are the normalized (by width of a single hole footprint) locations of the point and the base arcs with respect to the feature center. The values of $x_{\mathbf{O}_{i}}^{*}$ were selected based on of the methodology of Section 6.4.3. 


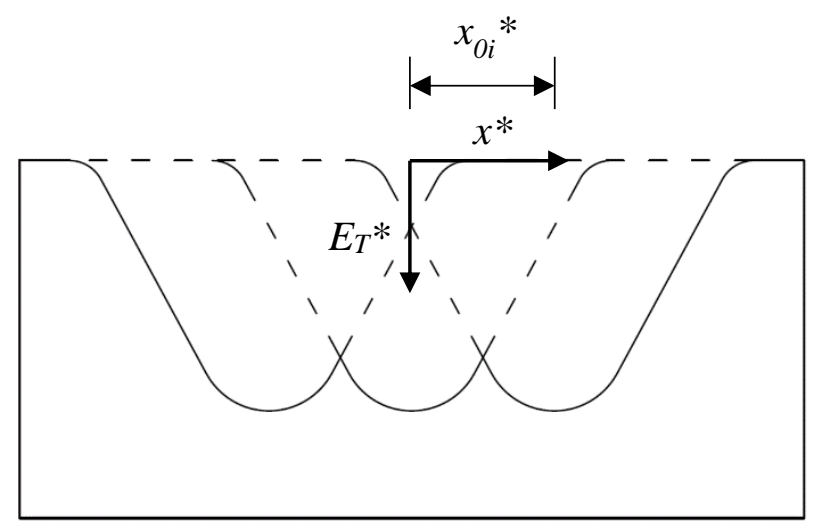

(a)

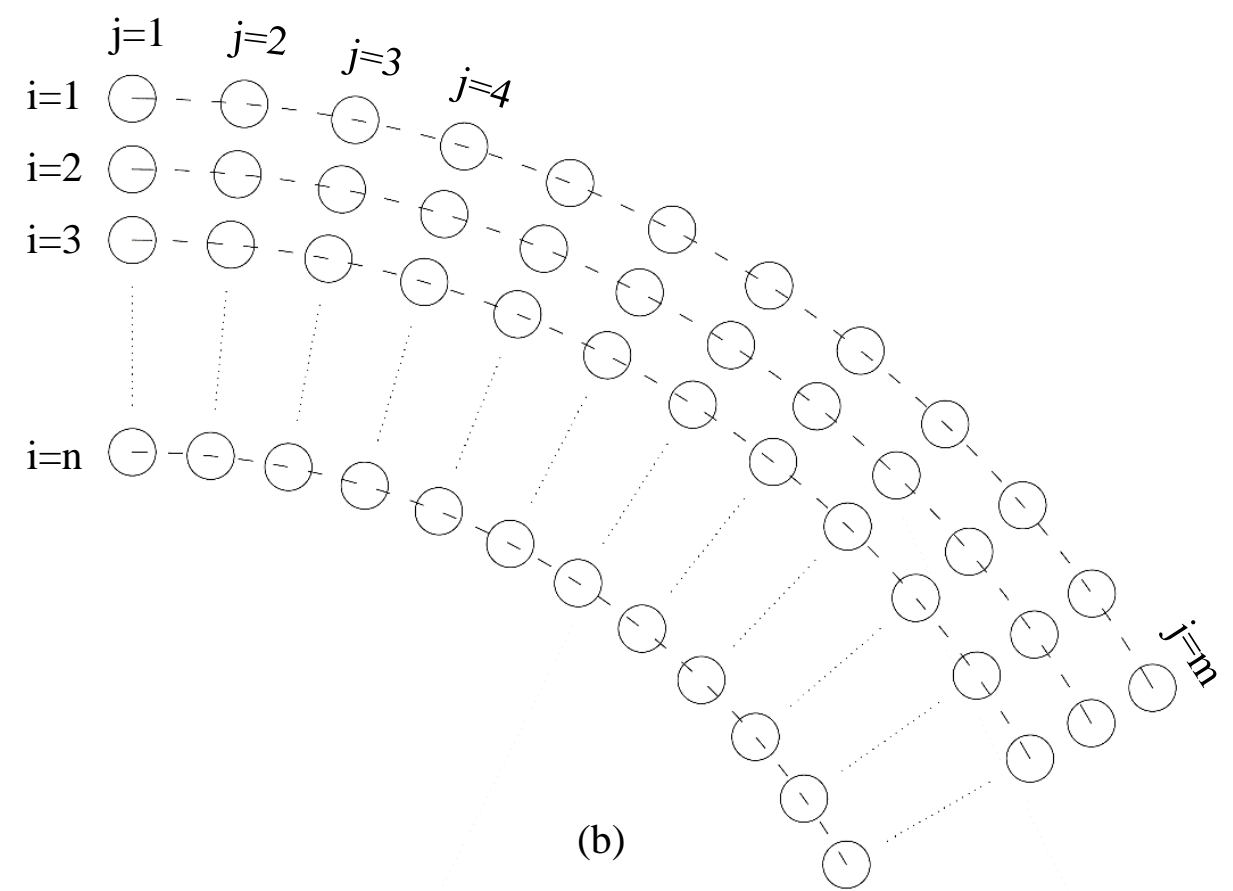

Figure 6-8 Schematic of (a) positive directions used for Eq. (6.4) and (b) " $i$ " and " $j$ " numbering for a $60^{\circ}$ portion of a pattern. " $\mathrm{n}$ " is the $\mathrm{n}^{\text {th }}$ base arc and " $\mathrm{m}$ " is the $\mathrm{m}^{\text {th }}$ hole on that arc.

\subsubsection{Determination of pattern needed to produce a desired erosive efficacy footprint}

The chosen number of base arcs, $i_{\max }$ to produce a desired erosive efficacy footprint strongly affected the fit of the resulting erosive efficacy to that desired. In general, the result is better with more base arcs, although there is an upper limit where additional base arcs become redundant (Section 6.4.4.7). Since there were four slots in the disk (Figure 6-2), adjacent base arcs 
could be placed over different slots, so that their proximity was not limited by the minimum bridge length before failure determined from the experiments of Section 6.2.6 (results in Section 6.4.3). In fact, they could be placed in this manner such that their associated erosive efficacies overlapped over a rotation. Nevertheless, $i_{\max }$ for a given hole size was constrained by the dimensions of the slots over which the masks were placed. Given this, the distance between two adjacent base arcs was typically $<0.5 \mathrm{~mm}$. For example, for a $2 \mathrm{~mm}$ slot width $i_{\max }=5$. With $i_{\text {max }}$ fixed in this manner, total number of holes that could be placed on any base arc was limited to $\sim 120$ by the $700 \mu \mathrm{m}$ minimum bridge length of Section 6.2.6. With this determined, the relative number of holes required on each base arc, taking into account the partial overlap of adjacent erosive efficacies, still needed to be determined. The problem reduces to determining set of parameters that minimizes the residual function $R^{*}$ representing the difference between Eq. (6.4) and the desired nondimensional efficacy footprint profile, $F^{*}\left(x^{*}\right)$, i.e.,

$$
R^{*}=\sum_{i=1}^{i_{\max }} \sum_{j=1}^{j_{\max }} d_{i, j}^{*} e^{-\beta_{i, j}^{2}\left(x^{*}+x_{0 i}^{*}\right)^{2}}-F^{*}\left(x^{*}\right)
$$

A nonlinear least square optimization method with "Trust region reflective" and "Levenberg-Marquardt" algorithms [99] was implemented in Matlab (The MathWorks, Inc., Natick, MA, USA) and used to solve this optimization problem for the required number of holes on each base arc. Values of $d_{i, j}$ and $\beta_{i, j}$ were both related to the size of the hole. These values were found by fitting a Gaussian curve over the footprint of a hole or a series of holes on one base arc (Section 6.3.1). The initial values of $i, j, d_{i, j}, \beta_{i, j}$ and $x_{0 i}$ were provided by the user. Based on the value of $R$ from Eq. (6.5), new values for those three variables were considered by the algorithm. This process was repeated by the software to approach the minimum value for $R$ resulting in the optimized parameters that gave the closest possible shape to the desired one. The stopping criteria for the optimization was when the difference in $R$ between two successive iterations was less than $10^{-6}$. The output were the oprimized $i_{\max }, j_{\max }, d_{i, j}, \beta_{i, j}$ and $x_{0 i}$.

For the simplified case where all the holes are assumed the same size, and the number of base arcs user specified, the problem reduces to optimizing the number of holes on each base arc $a_{i}$, i.e., 


$$
R^{*}=\sum_{i=1}^{i_{\max }} a_{i} d^{*} e^{-\beta^{2}\left(x^{*}+x_{0 i}^{*}\right)^{2}}-F^{*}\left(x^{*}\right)
$$

in which $a_{i}$ is the number of holes on the $i^{\text {th }}$ base arc.

In all cases, different initial guesses were used to initialize the optimization procedure. The maximum difference in $R^{*}$ using these initial guesses was always found to be $<1 \%$, indicating that the optimization converged to a global minimum.

\subsection{Results and discussion}

\subsubsection{Mask erosion}

Figure 6-9 shows that the durability (Section 6.2.4) of a hole on a mask attached to the target in the traditional manner was not significantly different ( $t$-test, $p$-value $=0.405)$ to that suspended on a stationary disk. The small difference may be due to different mechanisms of transferring the impact energy of the particles to the mask, i.e., for the case of the suspended mask, a portion of the impact energy was dissipated as a result of the mask flexing. On the rotating disk, the apparent durability of the suspended mask was much higher since the dose of particles incident to any point on the mask was much lower than in the other cases. In other words, the mask erosion was shared over the much larger circumferential area on a base arc, compared to a stationary hole that was exposed to all incoming particles. As a result, by using RMA it is possible to machine deeper channels, simply by increasing the radius of the base arc over which the pattern was printed to be able to fit more holes. 


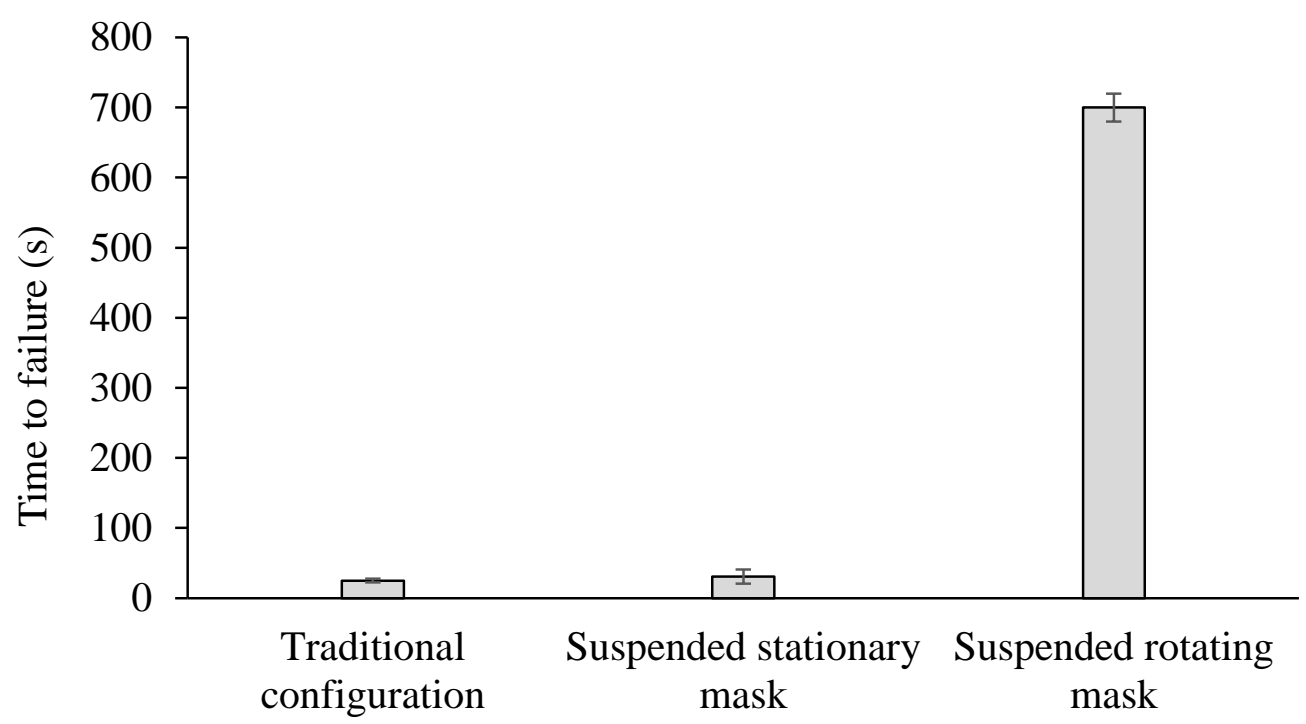

Figure 6-9 Mask durability for three configurations. Mass flow rate was $\sim 2.1 \mathrm{~g} / \mathrm{s}$ in all cases. The scatter bars represent \pm 1 standard deviation for 3 measurements.

\subsubsection{Effect of mask standoff on pattern transfer}

As Figure 6-10 shows, the pattern transfer resolution (Section 6.2.5) was very good in all cases, and only changed between 2 and $4 \%$ for mask standoffs between $0.3 \mathrm{~mm}$ and $1.5 \mathrm{~mm}$. Therefore, mask standoffs of $\sim 300 \mu \mathrm{m}$ were used in all other experiments.

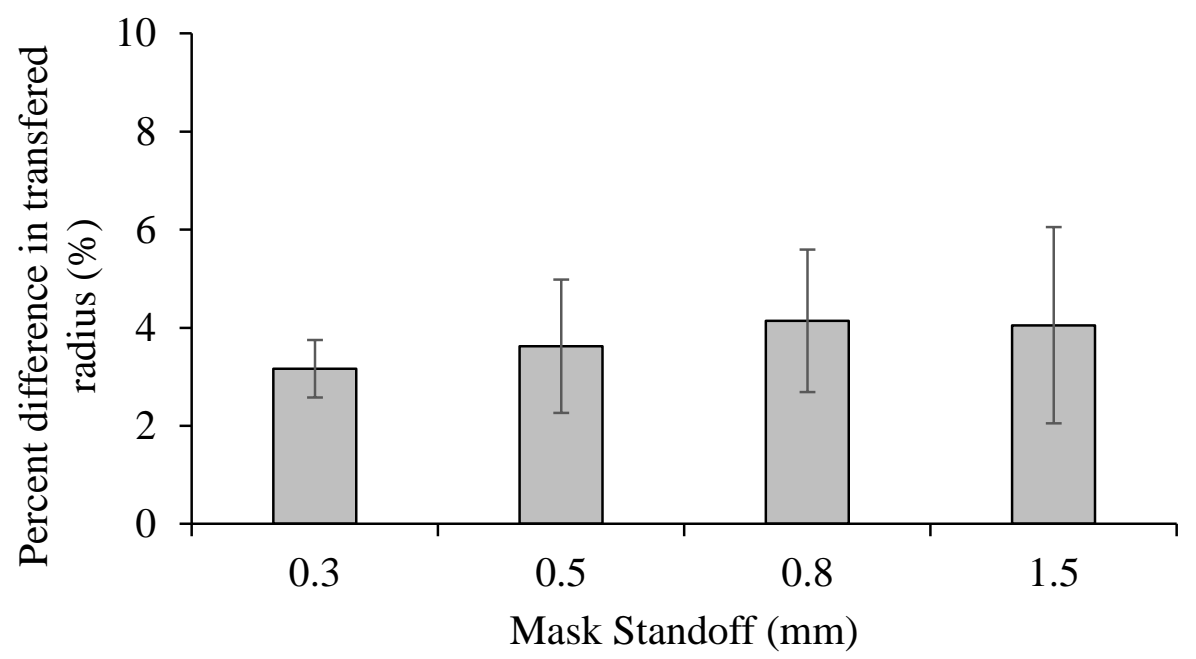

Figure 6-10 Effect of mask standoff on pattern transfer. The scatter bars represent \pm 1 standard deviation for 5 repetitions 


\subsubsection{Effect of distance between circles on mask}

Figure 6-11 shows three different modes of failure observed during the experiments described in Section 6.2.6. The first resulted in an abrupt tear in the mask between the two holes (Figure 6-11 (a)), and in the second, the 'bridge' between the holes eroded so that they connected (Figure 6-11 (b)). For a set of $300 \mu \mathrm{m}$ holes, these failures occurred for bridge lengths $<400 \mu \mathrm{m}$. In the third mode, the mask failed due to bulk erosion of the mask material (Figure 6-11 (c)). The first two failure modes are undesirable because they occur relatively rapidly, and can abruptly change the erosive efficacy that the target surface sees. The third mode is most desirable since it utilizes the maximum hole resistance and does not result in any abrupt change in resulting erosive efficacy during the machining.

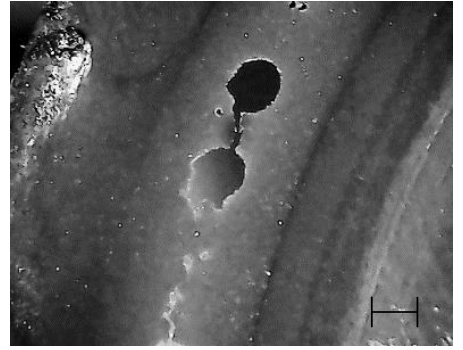

(a)

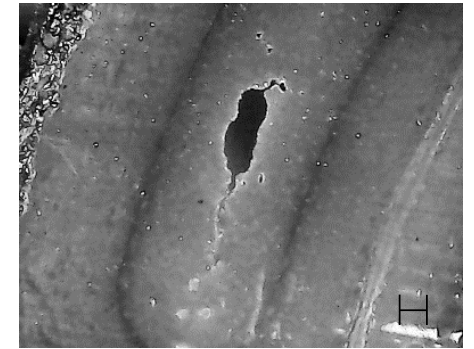

(b)

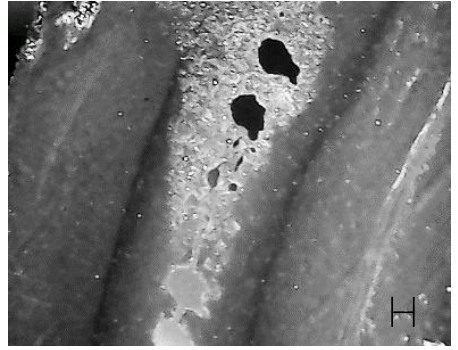

(c)

Figure 6-11 Three modes of failure in elastomeric mask. (a) Abrupt tear between two adjacent holes (bridge length=400 $\mu \mathrm{m}$ ). (b) Two holes became connected (bridge length=200 $\mu \mathrm{m}$ ). (c) failure of the mask material (bridge length=600 $\mu \mathrm{m}$ ). The scales on the figures are $300 \mu \mathrm{m}$.

Figure 6-12 shows that for a bridge length $>700 \mu \mathrm{m}$, the particle dose to failure was approximately constant at its maximum value, regardless of hole size. The experiments indicated that the eroded holes failed according to the gradual bulk erosion ( $3^{\text {rd }}$ mode described above). It is thus recommended that each hole be placed with a bridge length $>700 \mu \mathrm{m}$. 


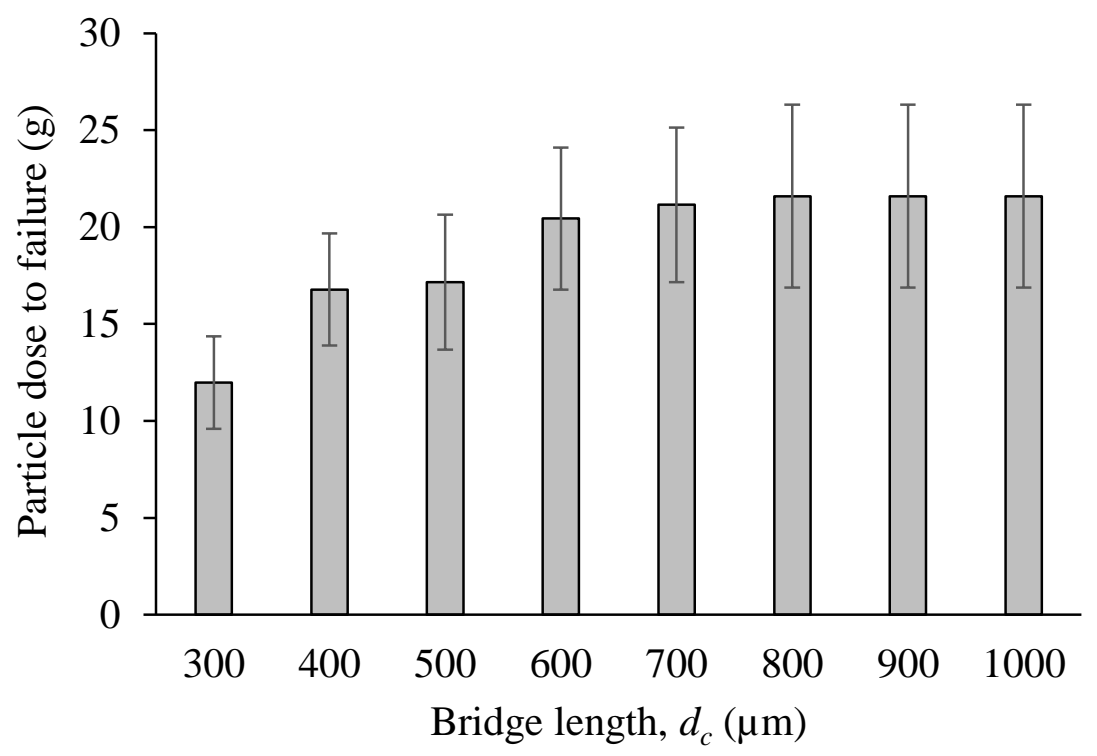

(a)

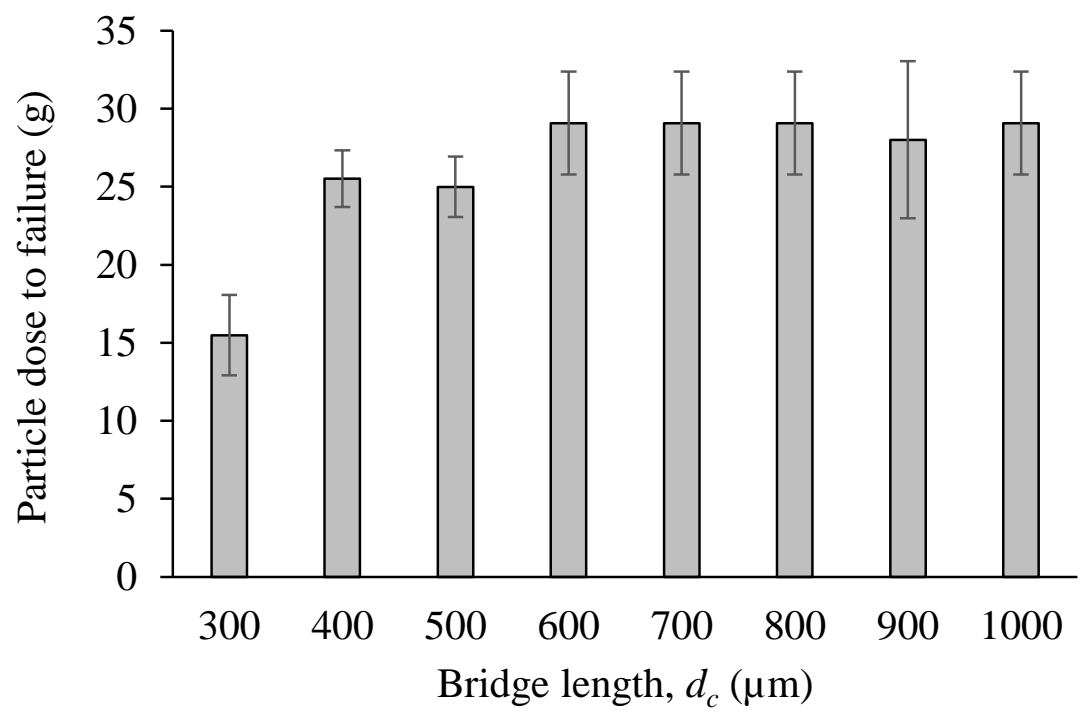

(b)

Figure 6-12 Average dose to failure from 3 repetition of the test for different bridge lengths of holes with diameter of (a) $300 \mu \mathrm{m}$ and (b) $600 \mu \mathrm{m}$. The scatter bars represent \pm 1 standard deviation for 3 measurements. 


\subsubsection{Machining of channels of various cross-sectional shapes}

As described in Section 6.2.8, different cross-sectional shapes were machined using the pattern prediction methodology of Section 6.3.3, and the results were compared to prediction of Eq. (6.4) in Section 6.3.2. The RMA was first used to illustrate that the sidewall slope on a typical $\mathrm{V}$ shaped channel could be controlled. Symmetric and asymmetric W-shaped micro channels were machined to illustrate that the RMA could be used to control the local shape of micro-channels, and that the overlap of adjacent erosive efficacies could be accurately predicted. Trapezoidal and wedge shape micro-channels were chosen as two practical examples of interest in microfluidics $[67,68,74]$ and to verify the model of Section 6.3 . The mask patterns used to machine each feature are given in Appendix B.

\subsubsection{Sidewall slope control}

Figure 6-13 compares the profiles of the 3 channels machined using $300 \mu \mathrm{m}, 600 \mu \mathrm{m}$, and alternating $300 \mu \mathrm{m}$ and $600 \mu \mathrm{m}$ holes. The difference in depth between the measured and predicted profiles was less than $7.2 \%$. To eliminate the effect of depth on the side wall slope comparison, each channel depth data point was normalized by dividing by the maximum depth. Although, for the same depth, the $300 \mu \mathrm{m}$ hole pattern was expected to result in a narrower channel with steeper sidewalls than the $600 \mu \mathrm{m}$, the combination was expected to produce an intermediate slope at the same width as that produced by the $600 \mu \mathrm{m}$ pattern. Indeed, Figure 6-13 shows that the normalized slope of combined holes $\left(77.2^{\circ}\right)$ was between the normalized slopes of the other two $\left(80.5^{\circ}\right.$ and $69.4^{\circ}$ for $300 \mu \mathrm{m}$ and $600 \mu \mathrm{m}$ respectively) for the case that the number of $300 \mu \mathrm{m}$ and $600 \mu \mathrm{m}$ holes were similar. Additional slopes can be obtained by simply changing the ratio between the two hole sizes on a given arc length. The solid line in Figure 6-13 shows that Eq. (6.4) can be used to effectively predict the resulting profile. 


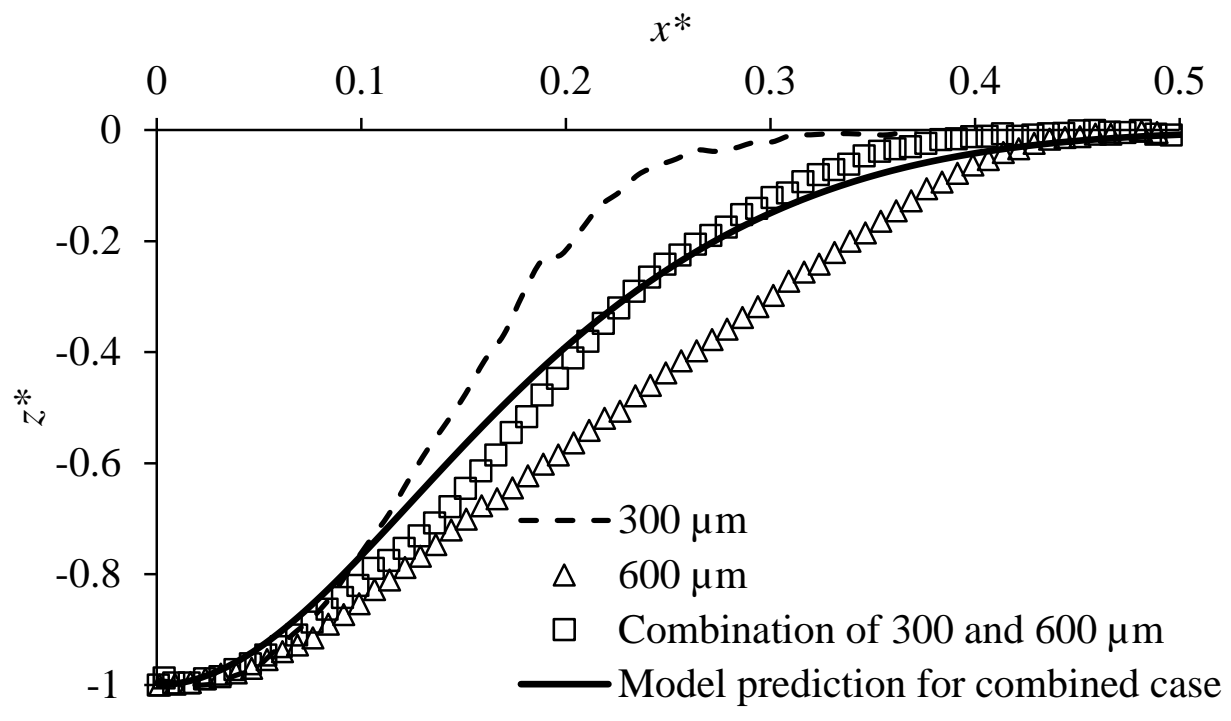

Figure 6-13 Comparison between side wall slopes of normalized channels (normalized by 872 $\mu \mathrm{m}$ in width) machined with hole diameters of $300 \mu \mathrm{m}, 600 \mu \mathrm{m}$ and the combination of two with equal number of holes from each. Solid line shows the prediction of Eq. (6.4).

\subsubsection{Symmetric W-shape}

The machined W-shapes illustrate how the instantaneous movement of an erosive efficacy source from one point to another can be simulated using the RMA with holes on multiple adjacent base arcs. They also investigate the assumption of Eq. (6.4), which was that the final shape could be found by adding the partially over-lapping erosive efficacies on adjacent base arcs. For example, it was desired to have a $\mathrm{W}$-shape erosive efficacy profile that was $900 \mu \mathrm{m}$ wide, with a center peak that was $80 \mu \mathrm{m}$ high. The obvious choice was to use patterns on two base arcs that partially overlapped. The optimization of Section 6.3.3 was used to determine the number of $300 \mu \mathrm{m}$ diameter holes on each base arc that were required. Figure 6-14 shows the resulting W-shaped channel machined using the RMA with this pattern. The difference between the depth of measured and predicted/desired (Eq. (6.4)) profiles was less than $9.1 \%$.

W-shaped channels were machined in Chapter 3 using oscillation of the target surface; however, that method only allowed a single maximum to minimum height ratio of $\sim 2$ (i.e. the ratio of the depth of the two sides to the depth of the center peak) to be achieved. The RMA, however, made it possible to have a full control over the size of the various peaks and valleys and channel width. For example, Figure 6-15 shows a $800 \mu \mathrm{m}$ wide W-shaped channel with smaller centre peak of $60 \mu \mathrm{m}$ which was achieved by decreasing the distance between the base arcs. 


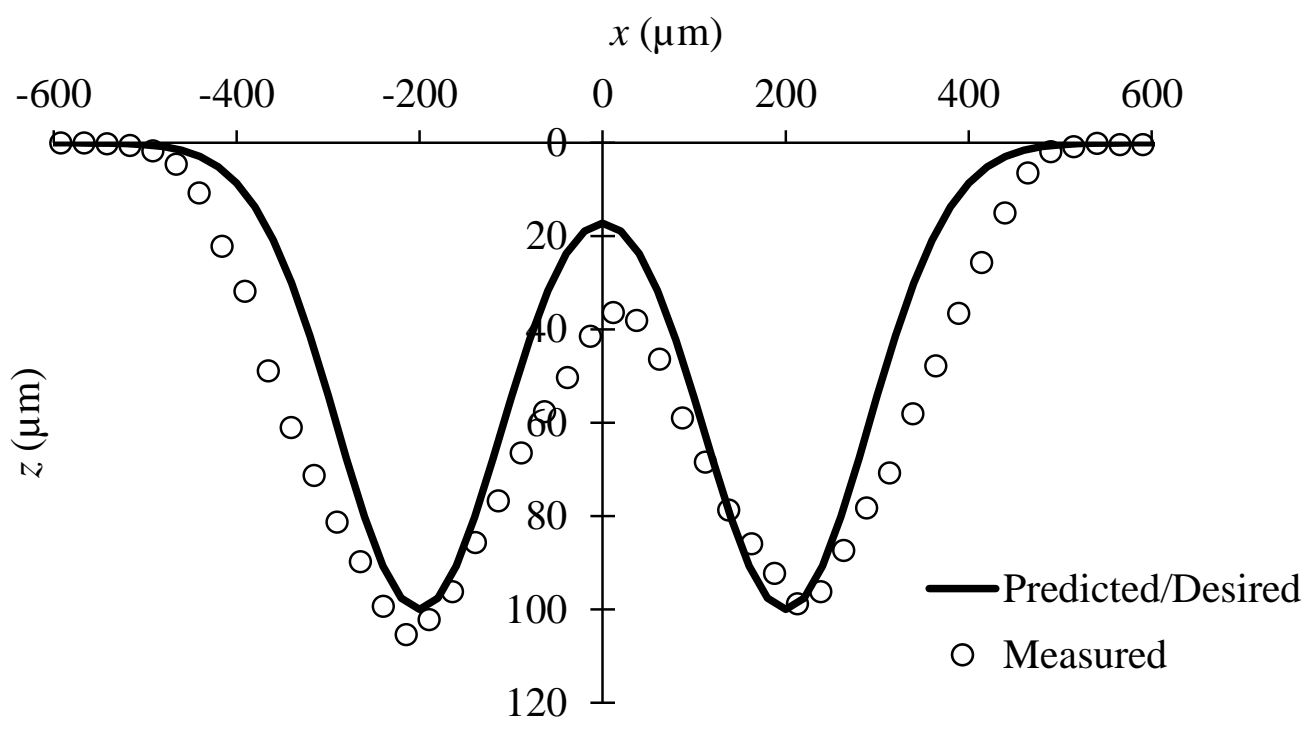

Figure 6-14 Profile of a symmetric W-shaped micro-channel. Two base arcs were used with radiuses of $20.3 \mathrm{~mm}$ and $20.7 \mathrm{~mm}$ each of which had 24 holes on it (mass flow rate=5.7 $\mathrm{g} / \mathrm{s}$ ).

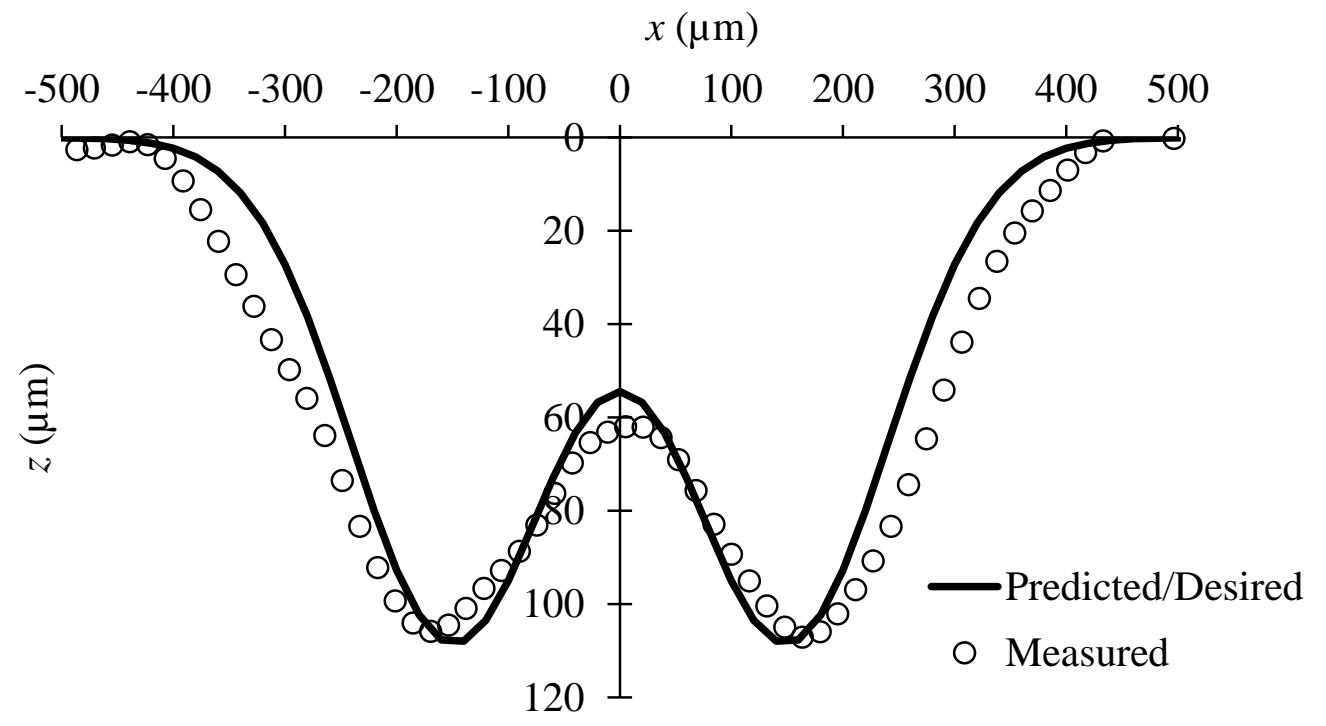

Figure 6-15 Profile of a symmetric W-shaped micro-channel. Two base arcs were used with radiuses of $20.35 \mathrm{~mm}$ and $20.65 \mathrm{~mm}$ each of which had 24 holes on it (mass flow rate=5.7 $\mathrm{g} / \mathrm{s}$ ).

\subsubsection{Asymmetric W-shape}

It was desired to machine an asymmetric $\mathrm{W}$-shape erosive efficacy profile that was 800 $\mu \mathrm{m}$ wide, with a depth difference of $40 \mu \mathrm{m}$ on the two valleys. Similar to Section 6.4.4.2, the 
obvious choice was to use patterns on two base arcs that partially overlapped. The optimization of Section 6.3.3 was used to determine the number of $300 \mu \mathrm{m}$ diameter holes on each base arc that were required (Appendix B). Figure 6-16 shows the resulting asymmetric W-shaped channel machined using the RMA with this pattern. The difference between the depth of measured and predicted/desired (Eq. (6.4)) profiles was less than $8.5 \%$.

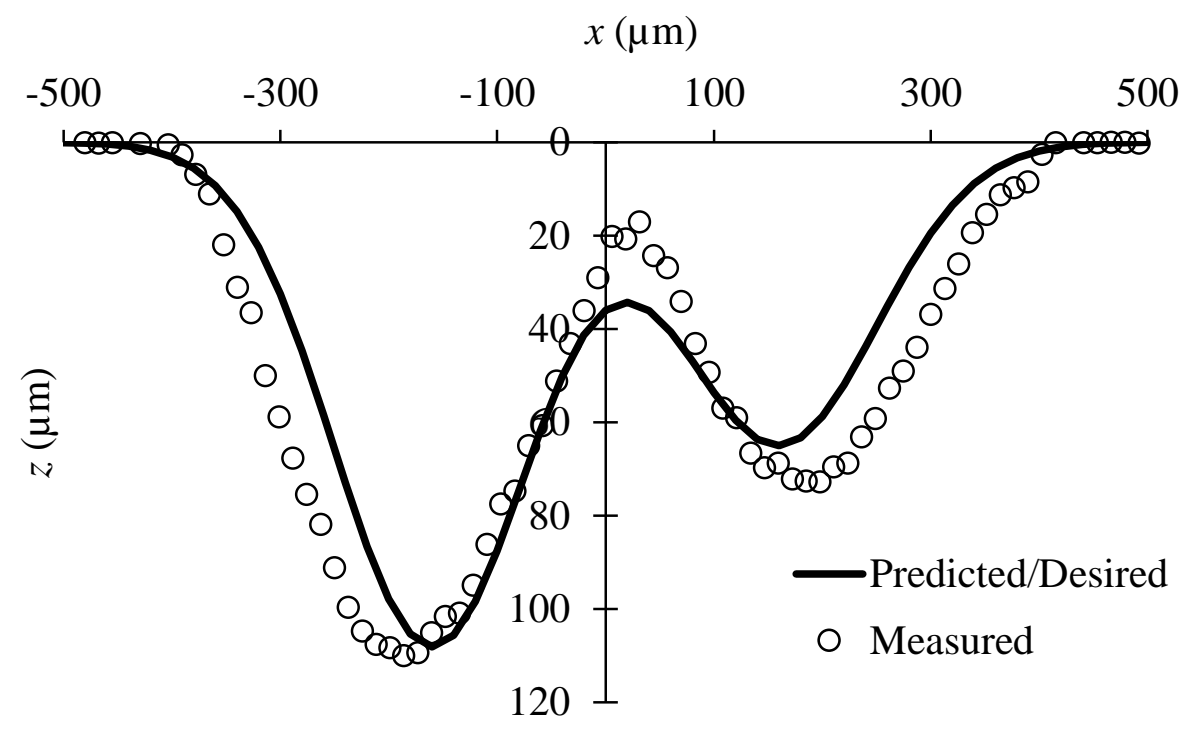

Figure 6-16 Profile of an asymmetric W-shaped micro-channel. Two base arcs were used with radiuses of $20.34 \mathrm{~mm}$ and $20.66 \mathrm{~mm}$ with 40 and 24 cured holes on them, respectively (mass flow rate $=5.6 \mathrm{~g} / \mathrm{s}$ ).

\subsubsection{Trapezoidal}

As discussed in Section 6.1, trapezoidal micro-channels have many applications in microfluidic devices. Using the RMA, it was possible to machine such a channel with full control over the width of the flat bottom and the side wall slope (Section 6.4.4.1). For example, it was desired to machine a $1600 \mu \mathrm{m}$ trapezoidal micro-channel with a $500 \mu \mathrm{m}$ wide flat surface in the middle. Following the results of Section 6.4 .3 and by examining different number of base arcs in Eq. (6.4), the optimum number of base arcs was found to be 5 for this case. The optimization of Section 6.3.3 was used to determine the number of $300 \mu \mathrm{m}$ diameter holes on each base arc that were required (Appendix B). Figure 6-17 shows the resulting trapezoidal micro-channel that was machined. The difference between the depth of measured and predicted/desired (Eq. (6.4)) profiles was less than $4.9 \%$. 


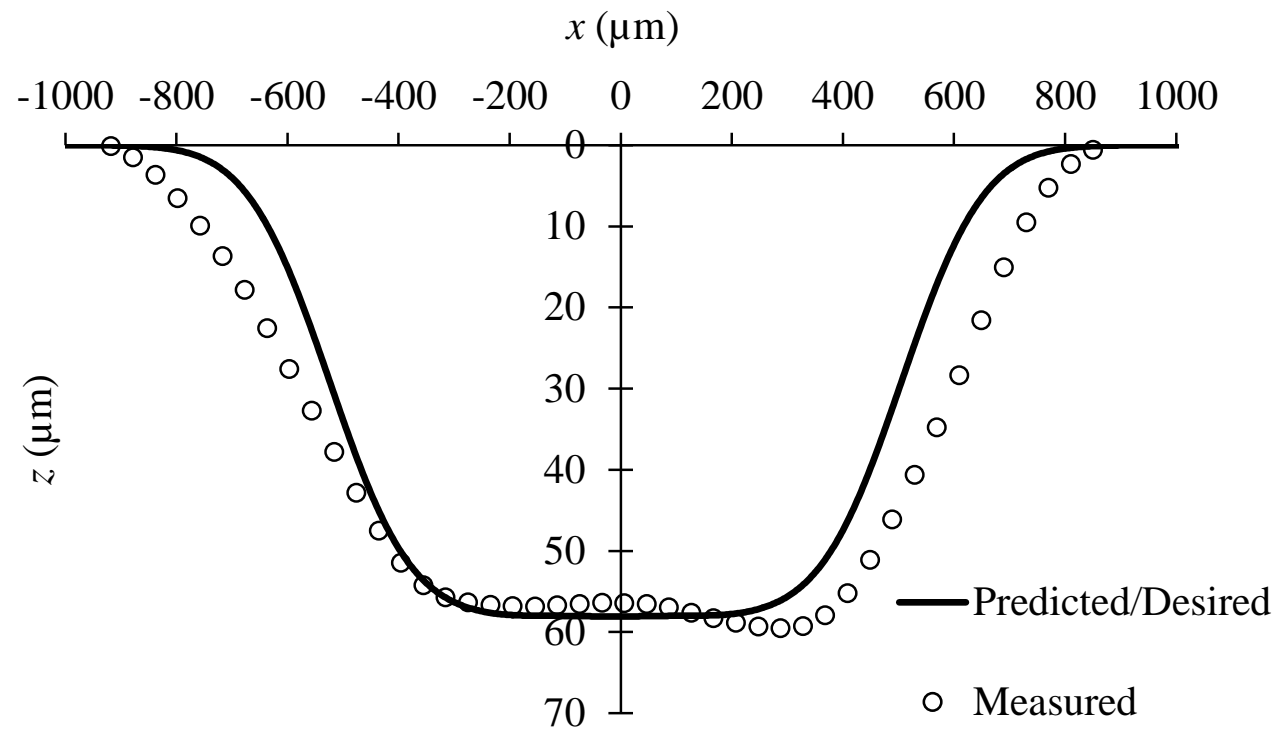

Figure 6-17 Normalized profile of a trapezoidal W-shaped micro-channel $\left(\mathrm{z}^{*}=\mathrm{z} / 59.9\right.$ and $\left.\mathrm{x}^{*}=\mathrm{x} / 575\right)$. Five base arcs were used with radiuses of $19.95 \mathrm{~mm}, 20.19 \mathrm{~mm}, 20.5 \mathrm{~mm}, 20.78 \mathrm{~mm}$ and $21.05 \mathrm{~mm}$ each of which had 16 holes on it (mass flow rate=4.6 g/s).

\subsubsection{Wedge shape}

Using the RMA, it was possible to machine a wedge shaped channel with full control over the width and the angle of the central portion. For example, it was desired to machine a $1600 \mu \mathrm{m}$ wedge shaped micro-channel with a $550 \mu \mathrm{m}$ wide wedge shaped surface in the middle. Similar to Section 6.4.4.4, and by following the results of Section 6.4.3 and Eq. (6.4), the optimum number of base arcs was found to be 5. The optimization of Section 6.3.3 was used to determine the number of $300 \mu \mathrm{m}$ diameter holes on each base arc that were required (Appendix B). Figure 6-18 shows the resulting wedge shaped micro-channel that was machined. The difference between the depth of measured and predicted/desired (Eq. (6.4)) profiles was less than $5.1 \%$. 


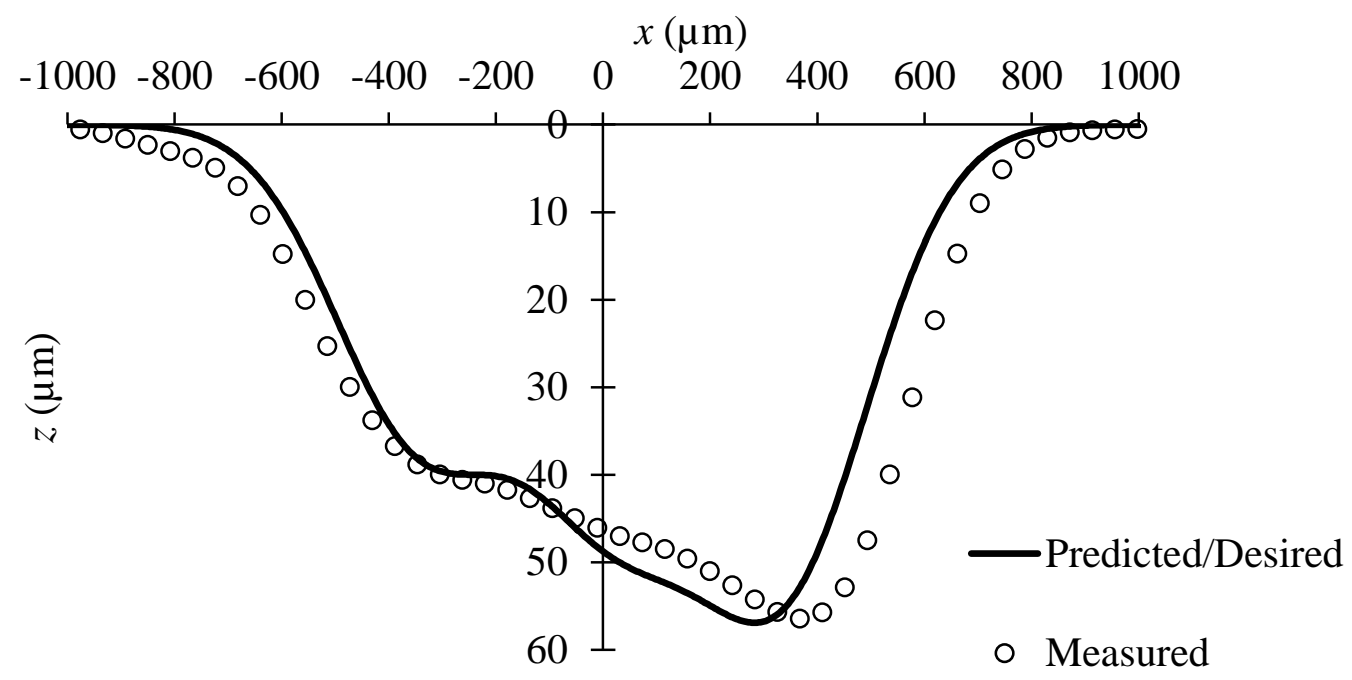

Figure 6-18 Profile of a trapezoidal W-shaped micro-channel. Five base arcs were used with radiuses of $19.95 \mathrm{~mm}, 20.19 \mathrm{~mm}, 20.5 \mathrm{~mm}, 20.78 \mathrm{~mm}$ and $21.05 \mathrm{~mm}$ with 20, 24, 28, 30 and 32 cured holes on them, respectively (mass flow rate $=4.2 \mathrm{~g} / \mathrm{s}$ ).

\subsubsection{Effect of base arc offset}

As mentioned in Section 6.2.3, the chosen number of base arcs and the resulting accuracy of a machined desired shape are closely related. If the number of chosen base arcs is insufficient, a wavy profile will result. For example, Figure 6-19 and Figure 6-20 show, respectively, examples of trapezoidal and wedge shaped channels that were desired to be $1600 \mu \mathrm{m}$ wide, machined using 3 base arcs. Although the optimization method of Section 6.3.3 was used to determine the number of $300 \mu \mathrm{m}$ diameter holes on each base arc, the number of base arcs was not adequate and the resulting profiles were wavy. Nevertheless, the proposed model (Eq. (6.4)) of Section 6.3.2 could still predict this waviness (solid line in Figure 6-19 and Figure 6-20). Therefore, Eq. (6.4) can be used with the optimization routine in a trial-and-error fashion to determine the number of base arcs necessary to produce a desired feature to any desired tolerance of waviness. To maintain an adequate amount of overlap of erosive efficacy from adjacent base arcs, the distance between them should not be greater than half of erosive efficacy of the holes. 


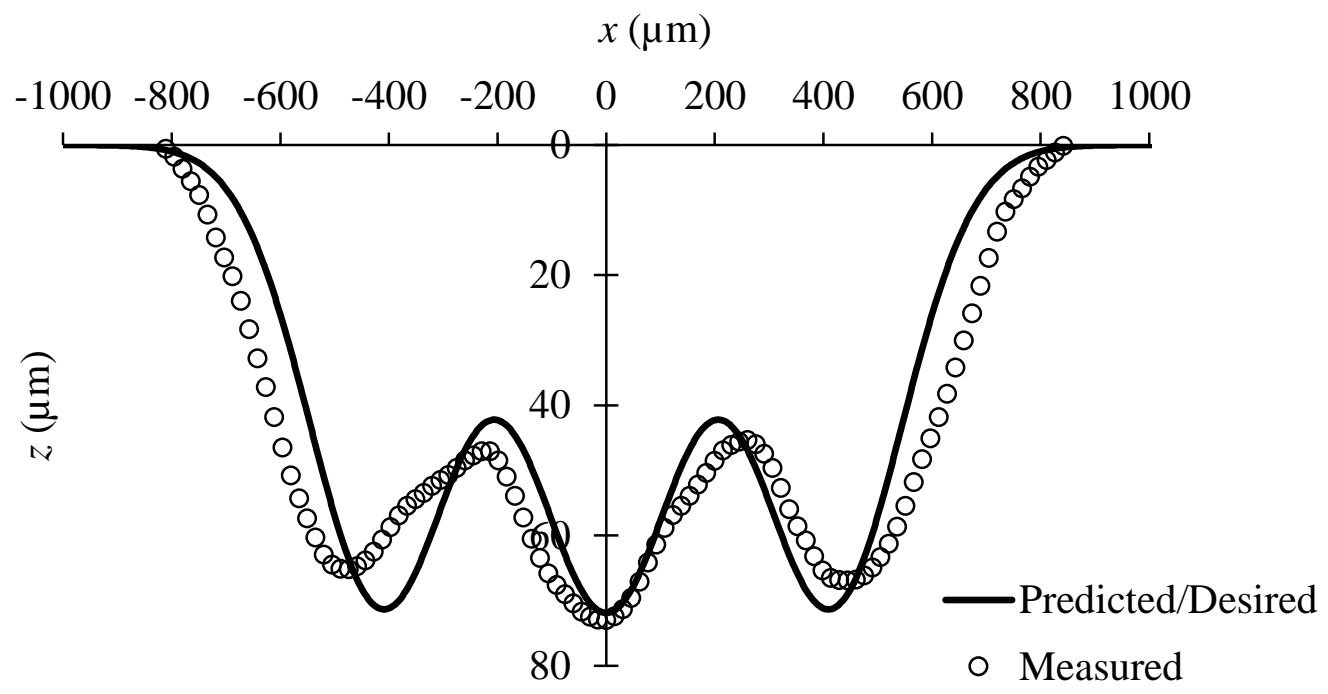

Figure 6-19 Profile of a wavy trapezoidal micro-channel. Three base arcs were used with radiuses of $19.95 \mathrm{~mm}, 20.5 \mathrm{~mm}$ and $21.05 \mathrm{~mm}$ each of which had 24 holes on it.

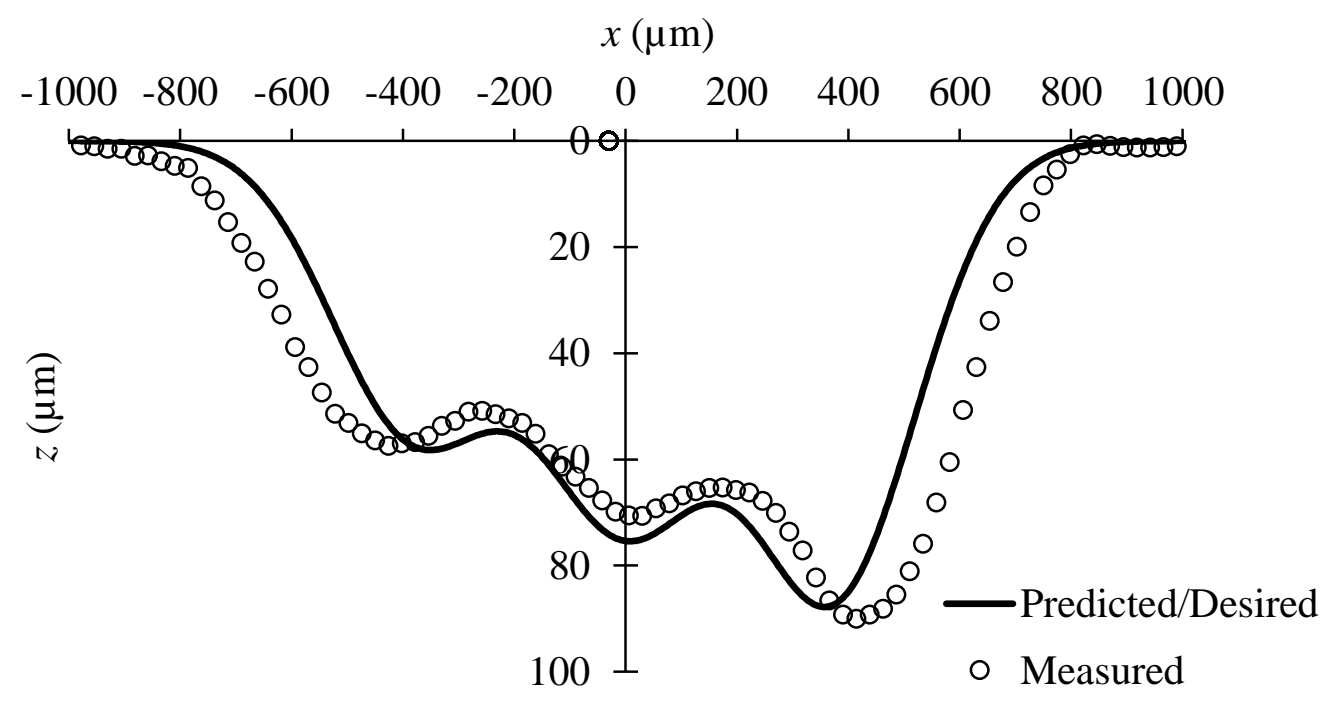

Figure 6-20 Profile of a wavy wedge shape micro-channel. Three base arcs were used with radiuses of $19.95 \mathrm{~mm}, 20.5 \mathrm{~mm}$ and $21.05 \mathrm{~mm}$ with 13,17 and 21 holes on them, respectively.

\subsubsection{Higher aspect ratio micro-channels}

The previous sections demonstrated how the RMA can be used to control the erosive efficacy footprint so that different shallow channel cross sectional shapes can be made. As mentioned earlier, one of the benefits of the RMA is its increased mask durability compared to traditional masks attached to a substrate. This also allows higher aspect ratio micro-features to be 
machined, as shown in the deeper trapezoidal and wedge shaped micro-channels of Figure 6-21. In both cases, five base arcs were considered. For the trapezoidal micro-channel, the optimization routine of Eq. (6.6) suggested zero holes on the $2^{\text {nd }}$ and $4^{\text {th }}$ base arcs, suggesting that machining on these arcs offers no additional benefit. This illustrates the benefits of using the optimization routine that could eliminate extra base arcs that were redundant. Although not shown, the final shape of these deeper channels could be predicted accurately using the predicted erosive efficacy of Eq. (6.4) in the surface evolution equations developed in previous work [3].

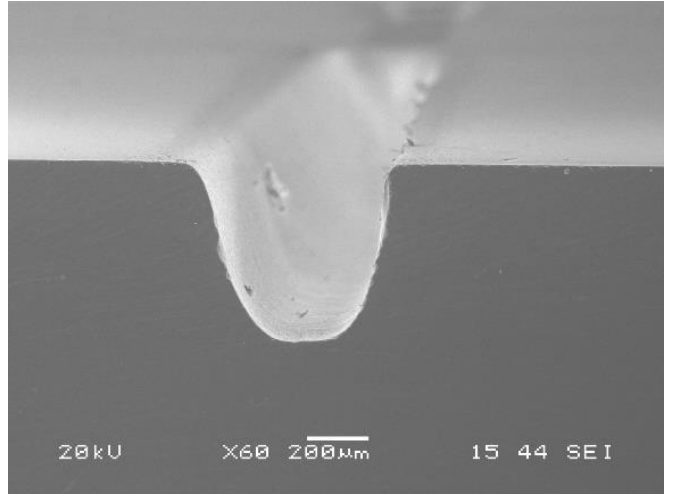

(a)

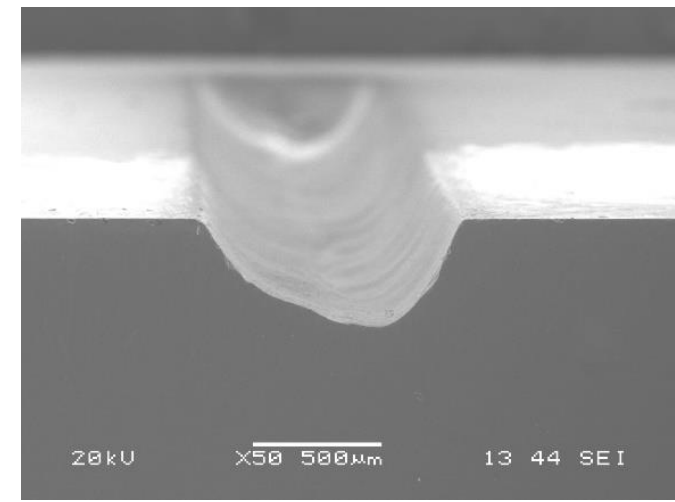

(b)

Figure 6-21 Higher aspect ratio narrow micro-channels machined using RMA with $200 \mu \mathrm{m}$ holes: (a) Trapezoidal channel using 3 base arcs with base arc radii of $20.4 \mathrm{~mm}, 20.5 \mathrm{~mm}$ and $20.6 \mathrm{~mm}$ (41, 14 and 41 holes on each arc). (b) Wedge shape microchannel using 5 base arcs with base arc radii of $20.1 \mathrm{~mm}, 20.3 \mathrm{~mm}, 20.5 \mathrm{~mm}, 20.7 \mathrm{~mm}$ and $20.9 \mathrm{~mm}(11,16,21,27$ and 32 holes on each arc).

\subsection{Summary}

A novel rotating mask apparatus (RMA) was developed to control the shape of the erosive efficacy reaching the surface, and therefore the cross-sectional shape of the resulting microchannels. The erosion of the suspended elastomeric masks that the RMA utilized was quantified and compared to traditional attached masks, and it was found the RMA configuration can increase the overall achievable machining time. An optimum mask stand-off was found to be approximately $300 \mu \mathrm{m}$. Criteria for designing the mask patterns were discussed and it was found that the minimum bridge length between holes should be $>700 \mu \mathrm{m}$ to avoid abrupt tearing and undesired connection of adjacent holes. 
A model was presented that could predict the final shape of shallow micro-channels machined using any mask design. The model was further used in an optimization code to predict the required pattern for machining any desired cross-sectional shape. The RMA apparatus was successfully used to control the side wall slope of the micro channels by changing the hole sizes on a single base arc. Based on the suggestions of the optimization routine, shallow symmetric Wshpae, asymmetric W-shape, trapezoidal and wedge shape micro-channels of desired topography were machined and the maximum error between the desired and produced topography was found to be less than $9.1 \%$ in all cases.

Overall, the results indicate that the RMA is a promising technique for the sculpting of the next generation of three-dimensional components for the micro-technology sector. The benefits of the RMA include: (i) the ability to generate a very wide range of erosive efficacies, including those that mimic moving a source (e.g. nozzle) or multiple sources of various sizes instantly from one location to another; (ii) improved mask longevity; and (iii) use of a single traverse in a straight line, rather than multiple adjacent passes, in order to sculpt desired 3D micro-feature shapes. 


\section{Chapter 7 Summary and Conclusions}

\subsection{Summary}

This dissertation was mainly focused on the problem of controlling and machining microchannels with desired and predictable shapes. In Chapter 2 an untraditional adjustable shadow mask was developed consisting of two parallel metal strips attached to a nozzle for the abrasive jet micro-machining of straight channels. The effects of nozzle standoff, mask standoff, particle size and mask thickness on the channel width, depth and size of the frosted region were investigated and could be explained in terms of changes in the particle velocity and impact location. Metal strips were also investigated in cross formation. It was demonstrated that this crossed-mask configuration had the additional advantage that it could also be used to direct write curved features.

A novel AJM oscillating target methodology to sculpt a variety of $\mathrm{U}, \mathrm{W}, \mathrm{V}$ and trapezoidal cross-sectional micro-channel shapes into brittle (glass) and ductile (PMMA) targets was presented and modeled in Chapter 3. Two techniques of obtaining a relatively small erosive efficacy footprint (maskless and shadow mask) were used with a sinusoidal target oscillation to create $\mathrm{W}$ and Vshaped cross sections. The W-shaped profiles could be further processed to obtain trapezoidal (glass), U-shaped (PMMA), and other unique cross sections.

Novel inverse methodologies were introduced in Chapter 4 to predict the instantaneous source velocity functions required to micro-machine, using both a small nozzle at a low standoff distance, and the shadow mask of Chapter 2, a wide variety of desired shallow feature profiles into brittle and ductile targets using gradient-etching AJM. The inverse methodology was demonstrated by example to have the potential to be used in a wide variety of applications, including sculpting desired channel cross-sectional shapes, lengthwise micro-channel texture, pocket texture, and combinations. The limits of applicability of the methodologies in terms of, e.g. relative source and feature size, were also established. Finally, methodologies for recreating visual representations of $2 \mathrm{D}$ and $3 \mathrm{D}$ textures were presented. This was the first time inverse methods have been used for path planning in AJM operations.

In Chapter 5, the velocities predicted by the methodologies of Chapter 4 were used to micro-machine a variety of desired features, and the resulting profiles were compared to the originally desired ones, in order to validate the approach of Chapter 4. Predicted (or desired) and 
measured channels with linearly, parabolically and sinusoidally changing depth along with pockets with texture in two directions were compared and verified to match within typically $7 \%$.

In Chapter 6 an innovative rotating mask apparatus (RMA) was developed to control the shape of the erosive efficacy reaching the surface, and therefore the cross-sectional shape of the resulting micro-channels. The erosion of the suspended elastomeric masks that the RMA utilized was quantified and compared to traditional attached masks. A model was presented that could predict the final shape of shallow micro channels machined using any mask design. The model was further used in an optimization code to predict the required pattern for machining any desired cross-sectional shape. The RMA apparatus was used to verify the model experimentally. Overall, the results indicated that the RMA is a promising technique for the sculpting of the next generation of three-dimensional components for the micro-technology sector.

\subsection{Conclusions}

The main conclusions of this dissertation are:

1. The shadow mask eliminates the need for costly and time consuming mask fabrication and attachment to the substrate, and allows for AJM to be used as a direct write microfabrication technology. Using the shadow mask, it was possible to machine micro-channels that were only approximately $10 \%$ wider than those machined using traditional masks up to an aspect ratio of approximately 0.5. By using two sets of crossed shadow masks, it was possible to directly write and machine curved channels with any desired pattern in the substrate.

2. A target oscillation technique was shown to be useful for machining W-shaped microchannels. The scan speed, oscillation amplitude and size of the source erosive efficacy were the main parameters that affected their shape. Previously developed surface evolution models could be used with an analytically predicted erosive efficacy to successfully predict their shape with less than $7 \%$ error. The $\mathrm{W}$-shaped profiles could be transformed into trapezoidal or U-shaped ones by performing additional passes along their centers, using an appropriate scan speed. 
3. For a Gaussian erosive efficacy distribution, a closed form solution was found to the inverse problem of finding the required velocity function to machine a desired eroded profile. For non-Gaussian sources, there is no closed form solution and an approximate approach assuming a point source must be used. The inverse methodology has the potential to be used in a wide variety of applications, including sculpting desired channel crosssectional shapes, lengthwise micro-channel texture, pocket texture, and combinations. Typically, the maximum error between the measured topographies on features machined using the predicted velocities, and the originally desired topographies was less than $7 \%$.

4. The RMA apparatus introduced in this thesis was found to be able to generate a very wide range of erosive efficacies, including those that mimic moving a source (e.g. nozzle) or multiple sources of various sizes instantly from one location to another. Together with the presented optimization methodology, it allows virtually any desired micro-channel crosssectional topography to be machined using AJM. It also allows predictable control of micro-channel side wall slope by a simple change in pattern hole size. For example, it was shown that the mask patterns required to machine symmetric and asymmetric W-shape, trapezoidal and wedge shape micro-channels could be predicted such that the resulting etched topography agreed well with that desired ( $<9.1 \%$ error in all cases). Because it can sculpt desired 3D micro-feature shapes in a single pass, the RMA decreases the required machining time compared to traditional methods which overlap adjacent passes. It also increases the maximum machining time before masks wear out, since multiple masks are used to share the erosion. The elastomeric mask patterns, however, must have a minimum bridge length between holes of $>700 \mu \mathrm{m}$ to avoid abrupt tearing and undesired connection of adjacent holes. 


\subsection{Contributions}

The novel contributions of this dissertation can be summarized as:

1. A novel shadow mask apparatus was presented and tested for machining straight microchannels and direct write using parallel and crossed mask, respectively.

2. A new definition of channel width was presented which made it possible to distinguish between width and edge frosting.

3. For the first time, inverse methods were presented that allowed prediction of the machining path necessary to produce a given desired feature shape using AJM. This was demonstrated by machining a wide variety of desired textures and cross sectional shapes into microchannels and pockets.

4. A novel rotating mask apparatus was introduced that could be used to mimic virtually any movement of a source, including near-instantaneous movement from one point to another, something that has previously not been possible.

5. A novel optimization method was developed and verified that allowed prediction of the rotating mask pattern required to machine a desired cross-sectional feature shape. Because of its unprecedented flexibility in creating a wide variety of micro-channel topographies, the device is extremely promising for the rapid prototyping of novel microfluidic devices.

6. As part of the rotating mask development, the erosion resistance of Rapid Mask selfadhesive masks was characterized to an unprecedented degree in a wide variety of configurations.

\subsection{Recommendations for future work}

There is potential to extend the work in this dissertation in the following ways:

I. The design of the shadow mask should be improved to allow mounting the nozzle at oblique angles of attack and to have finer control over the mask opening. This would make it easier to machine asymmetric features, and extend the variety of machinable features. For example, more abrupt changes in channel depth (higher slopes in the machining direction) could be achieved by a masked oblique incident jet. 
II. The inverse solution models presented in Chapter 4 for prediction of the machining traverse velocity and path could be extended to abrasive water and slurry jet machining. Because of the complex flow of liquid slurries in machined features, this would most likely require the results of computational fluid dynamics to be implemented in the models.

III. The inverse techniques can be improved to predict desired high aspect ratio shapes. For deeper channels, the surface evolution equation needs to be solved in order to predict the required erosive efficacy. This can be done by introducing an optimization routine to minimize the difference between surface-evolution-modeled and desired profiles of deep channels (a residual function).

IV. The inverse solution models could be used to remove (i.e., smooth) roughness and waviness from micro-channels machined using abrasive water jets, which are particularly prone to process fluctuations. In such a scheme, the center line profile of the wavy micro-channels would be obtained using profilometer, and the inverse method would then be used to predict the velocity required to remove the waviness without significantly impacting the channel depth and shape.

V. The RMA apparatus could be modified to use metallic masks patterned (perhaps using laser micro-machining) with pre-aligned hole patterns. This would allow a higher mask erosion resistance in order to machine higher aspect ratio channels of non-traditional shape.

VI. The RMA could be used with varying target speed in order to create texture along the length of channels with any desired cross-sectional shape. This would require first finding the appropriate disk mask pattern using the optimization methods presented in Chapter 6 (to create the channel cross sectional profile) and then solving the inverse problem of Chapter 4 to find the scan velocity required for the desired longitudinal texture. 


\section{Appendices}

Appendix A Coefficients of Fourier series for features in Table 4-1

\begin{tabular}{|c|c|c|c|c|c|c|c|c|c|c|c|c|c|c|}
\hline & \multicolumn{2}{|c|}{ Linear } & \multicolumn{3}{|c|}{$\underline{\text { Parabolic }}$} & \multicolumn{3}{|c|}{$\underline{\text { Sinusoidal }}$} & \multicolumn{3}{|c|}{ Half circle } & \multicolumn{3}{|c|}{ Inversed half circle } \\
\hline$a_{0}$ & $a_{n}$ & $b_{n}$ & $a_{0}$ & $a_{n}$ & $b_{n}$ & $a_{0}$ & $a_{n}$ & $b_{n}$ & $a_{0}$ & $a_{n}$ & $b_{n}$ & $a_{0}$ & $a_{n}$ & $b_{n}$ \\
\hline \multirow[t]{15}{*}{2.00} & 0.00 & 0.64 & \multirow[t]{15}{*}{-0.67} & 1.62 & 2.55 & \multirow[t]{15}{*}{4.00} & 0.00 & 0.00 & \multirow[t]{15}{*}{8.07} & 2.48 & -2.83 & \multirow[t]{15}{*}{5.93} & -2.48 & 2.83 \\
\hline & 0.00 & -0.32 & & -0.41 & -1.27 & & 0.00 & 1.00 & & 0.71 & -1.90 & & -0.71 & 1.90 \\
\hline & 0.00 & 0.21 & & 0.18 & 0.85 & & 0.00 & 0.00 & & 0.35 & -0.47 & & -0.35 & 0.47 \\
\hline & 0.00 & -0.16 & & -0.10 & -0.64 & & 0.00 & 0.00 & & 0.27 & -0.99 & & -0.27 & 0.99 \\
\hline & 0.00 & 0.13 & & 0.07 & 0.51 & & 0.00 & 0.00 & & 0.15 & -0.21 & & -0.15 & 0.21 \\
\hline & 0.00 & -0.11 & & -0.05 & -0.42 & & 0.00 & 0.00 & & 0.15 & -0.68 & & -0.15 & 0.68 \\
\hline & 0.00 & 0.09 & & 0.03 & 0.36 & & 0.00 & 0.00 & & 0.09 & -0.13 & & -0.09 & 0.13 \\
\hline & 0.00 & -0.08 & & -0.03 & -0.32 & & 0.00 & 0.00 & & 0.10 & -0.51 & & -0.10 & 0.51 \\
\hline & 0.00 & 0.07 & & 0.02 & 0.28 & & 0.00 & 0.00 & & 0.06 & -0.09 & & -0.06 & 0.09 \\
\hline & 0.00 & -0.06 & & -0.02 & -0.26 & & 0.00 & 0.00 & & 0.07 & -0.42 & & -0.07 & 0.42 \\
\hline & 0.00 & 0.06 & & 0.01 & 0.23 & & 0.00 & 0.00 & & 0.04 & -0.06 & & -0.04 & 0.06 \\
\hline & 0.00 & -0.05 & & -0.01 & -0.21 & & 0.00 & 0.00 & & 0.05 & -0.35 & & -0.05 & 0.35 \\
\hline & 0.00 & 0.05 & & 0.01 & 0.20 & & 0.00 & 0.00 & & 0.03 & -0.05 & & -0.03 & 0.05 \\
\hline & 0.00 & -0.05 & & -0.01 & -0.18 & & 0.00 & 0.00 & & 0.04 & -0.30 & & -0.04 & 0.30 \\
\hline & 0.00 & 0.04 & & 0.01 & 0.17 & & 0.00 & 0.00 & & 0.02 & -0.04 & & -0.02 & 0.04 \\
\hline
\end{tabular}


Appendix B RMA pattern arrangement for different cross sectional profiles

\begin{tabular}{lll} 
Cross sectional profile & Number of base arcs & Number of holes \\
\hline Asymmetric W-shape & 2 & 40,24 \\
Symmetric W-shape & 2 & 24,24 \\
Trapezoidal & 5 & $16,16,16,16,16$ \\
Trapezoidal (narrow) & 3 & $41,14,41$ \\
Wedge shape & 5 & $20,24,28,30,32$ \\
Wedge shape (narrow) & 5 & $11,16,21,27,32$ \\
Wavy trapezoidal & 3 & $24,24,24$ \\
Wavy wedge shape & 3 & $13,17,21$ \\
\hline
\end{tabular}




\section{References}

[1] T. Burzynski, M. Papini, Level set methods for the modelling of surface evolution in the abrasive jet micromachining of features used in MEMS and microfluidic devices, J. Micromechanics Microengineering. 20 (2010) 85004. doi:10.1088/0960-1317/20/8/085004.

[2] S. Ghosal, Lubrication theory for electro-osmotic flow in a microfluidic channel of slowly varying cross-section and wall charge, J. Fluid Mech. 459 (2002) 103-128. doi:10.1017/S0022112002007899.

[3] J.H.M. ten Thije Boonkkamp, P.J. Slikkerveer, Mathematical modelling of erosion by powder blasting, Surv. Math. Ind. 10 (2000) 89-106.

[4] J. Bitter, A study of erosion phenomena part I, Wear. 6 (1963) 5-21.

[5] J. Bitter, A study of erosion phenomena part II, Wear. 6 (1963) 169-190.

[6] D.R. Andrews, An analysis of solid particle erosion mechanisms, J Phys Appl Phys. 14 (1981) 1979-1991. doi:10.1088/0022-3727/14/11/006.

[7] I. Finnie, Erosion of surfaces by solid particles, Wear. 3 (1960) 87-103.

[8] I.M. Hutchings, Deformation of metal-surfaces by oblique impact of square plates, Int J Mech Sci. 19 (n.d.) 45.

[9] M. Papini, J.K. Spelt, Impact of rigid angular particles with fully-plastic targets Part I: Analysis, Int. J. Mech. Sci. 42 (2000) 991-1006. doi:10.1016/S0020-7403(99)00023-5.

[10] M. Papini, J.K. Spelt, Impact of rigid angular particles with fully-plastic targets Part II: Parametric study of erosion phenomena, Int. J. Mech. Sci. 42 (2000) 1007-1025. doi:10.1016/S0020-7403(99)00024-7.

[11] M. Takaffoli, M. Papini, Finite element analysis of single impacts of angular particles on ductile targets, Wear. 267 (2009) 144-151. doi:10.1016/j.wear.2008.10.004.

[12] M. Takaffoli, M. Papini, Material deformation and removal due to single particle impacts on ductile materials using smoothed particle hydrodynamics, Wear. 274-275 (2012) 50-59. doi:10.1016/j.wear.2011.08.012.

[13] M. Takaffoli, M. Papini, Numerical simulation of solid particle impacts on Al6061-T6 part I: Three-dimensional representation of angular particles, Wear. 292-293 (2012) 100-110. doi:10.1016/j.wear.2012.05.028.

[14] M. Takaffoli, M. Papini, Numerical simulation of solid particle impacts on Al6061-T6 Part II: Materials removal mechanisms for impact of multiple angular particles, Wear. 296 (2012) 648-655. doi:10.1016/j.wear.2012.07.022.

[15] I.M. Hutchings, R.E. Winter, J.E. Field, Solid Particle Erosion of Metals: The Removal of Surface Material by Spherical Projectiles, Proc. R. Soc. Math. Phys. Eng. Sci. 348 (1976) 379-392. doi:10.1098/rspa.1976.0044.

[16] V. Jardret, H. Zahouani, J.L. Loubet, T.G. Mathia, Understanding and quantification of elastic and plastic deformation during a scratch test, Wear. 218 (1998) 8-14. doi:10.1016/S0043-1648(98)00200-2.

[17] D.G. Rickerby, N.H. MacMillan, The erosion of aluminum by solid particle impingement at normal incidence, Wear. 60 (1980) 369-382. doi:10.1016/0043-1648(80)90235-5.

[18] Y. Oka, M. Matsumura, T. Kawabata, Relationship between surface hardness and erosion damage caused by solid particle impact, Wear. 162 (1993) 688-695.

[19] Y.I. Oka, K. Okamura, T. Yoshida, Practical estimation of erosion damage caused by solid particle impact Part 1: Effects of impact parameters on a predictive equation, Wear. 259 (2005) 95-101. 
[20] Y.I. Oka, T. Yoshida, Practical estimation of erosion damage caused by solid particle impact: Part 2: Mechanical properties of materials directly associated with erosion damage, Wear. 259 (2005) 102-109. doi:10.1016/j.wear.2005.01.040.

[21] I. Finnie, D.H. McFadden, On the velocity dependence of the erosion of ductile metals by solid particles at low angles of incidence, Wear. 48 (1978) 181-190. doi:10.1016/00431648(78)90147-3.

[22] D.R. Andrews, J.E. Field, Temperature dependence of the impact response of copper: erosion by melting, J. Phys. Appl. Phys. 15 (1982) 2357. doi:10.1088/0022-3727/15/11/027.

[23] N. P Suh, The delamination theory of wear, Wear. 25 (1973) 111-124.

[24] S. Jahanmir, The mechanics of subsurface damage in solid particle erosion, Wear. 61 (1980) 309-324. doi:10.1016/0043-1648(80)90294-X.

[25] I. Finnie, Some Reflections on the Past and Future of Erosion, Wear. 186-187 (1995) 1-10. doi:10.1016/0043-1648(95)07188-1.

[26] H. Wensink, M.C. Elwenspoek, A closer look at the ductile-brittle transition in solid particle erosion, Wear. 253 (2002) 1035-1043.

[27] P.J. Slikkerveer, P.C.P. Bouten, F.H. in't Veld, H. Scholten, Erosion and damage by sharp particles, Wear. 217 (1998) 237-250. doi:10.1016/S0043-1648(98)00187-2.

[28] A.G. Pawlowski, A. Sayah, M.A.M. Gijs, Accurate masking technology for high-resolution powder blasting, J. Micromechanics Microengineering. 15 (2005) 60.

[29] H. Wensink, H.V. Jansen, J.W. Berenschot, M.C. Elwenspoek, Mask materials for powder blasting, J. Micromechanics Microengineering. 10 (2000) 175-180.

[30] Y.S. Liao, L.C. Chen, A method of etching and powder blasting for microholes on brittle materials, J. Mater. Process. Technol. 209 (2009) 4390-4394. doi:10.1016/j.jmatprotec.2008.11.032.

[31] a Ghobeity, H. Getu, M. Papini, J.K. Spelt, Surface evolution models for abrasive jet micromachining of holes in glass and polymethylmethacrylate (PMMA), J. Micromechanics Microengineering. 17 (2007) 2175-2185. doi:10.1088/0960-1317/17/11/003.

[32] T. Burzynski, M. Papini, A level set methodology for predicting the effect of mask wear on surface evolution of features in abrasive jet micro-machining, J. Micromechanics Microengineering. 22 (2012) 75001. doi:10.1088/0960-1317/22/7/075001.

[33] A. Ghobeity, H.J. Crabtree, M. Papini, J.K. Spelt, Characterisation and comparison of microfluidic chips formed using abrasive jet micromachining and wet etching, J. Micromechanics Microengineering. 22 (2012) 25014. doi:10.1088/0960-1317/22/2/025014.

[34] M. Achtsnick, J. Drabbe, A.M. Hoogstrate, B. Karpuschewski, Erosion behaviour and pattern transfer accuracy of protecting masks for micro-abrasive blasting, J. Mater. Process. Technol. 149 (2004) 43-49. doi:10.1016/j.jmatprotec.2003.10.037.

[35] J.H.M. Ten Thije Boonkkamp, J.K.M. Jansen, An analytical solution for mechanical etching of glass by powder blasting, J. Eng. Math. 43 (2002) 385-399. doi:10.1023/A:1020359220269.

[36] P.J. Slikkerveer, F.H.I. Veld, Model for patterned erosion, Wear. 233-235 (1999) 377-386. doi:10.1016/S0043-1648(99)00177-5.

[37] H. Getu, A. Ghobeity, J.K. Spelt, M. Papini, Abrasive jet micromachining of polymethylmethacrylate, Wear. 263 (2007) 1008-1015. doi:10.1016/j.wear.2007.01.063.

[38] A. Ghobeity, M. Papini, J.K. Spelt, Computer simulation of particle interference in abrasive jet micromachining, Wear. 263 (2007) 265-269. doi:10.1016/j.wear.2007.01.112. 
[39] A. Ghobeity, T. Krajac, T. Burzynski, M. Papini, J.K. Spelt, Surface evolution models in abrasive jet micromachining, Wear. 264 (2008) 185-198. doi:10.1016/j.wear.2007.02.020.

[40] H. Yagyu, O. Tabata, Micro-powder blasting simulation with mask erosion using cellular automaton, 2007 IEEE 20th Int. Conf. Micro Electro Mech. Syst. MEMS. 2 (2007) 215-218. doi:10.1109/MEMSYS.2007.4433139.

[41] N. Shafiei, Computer Simulation of Developing Erosion Profiles including Interference Effects [MASc thesis], Ryerson University, 2008.

[42] D. Ciampini, M. Papini, A cellular automata and particle-tracking simulation of abrasive jet micromachining that accounts for particle spatial hindering and second strikes, J. Micromechanics Microengineering. 20 (2010) 45025. doi:10.1088/0960-1317/20/4/045025.

[43] A. Ghobeity, D. Ciampini, M. Papini, An analytical model of the effect of particle size distribution on the surface profile evolution in abrasive jet micromachining, J. Mater. Process. Technol. 209 (2009) 6067-6077. doi:10.1016/j.jmatprotec.2009.05.026.

[44] E. Belloy, A. Sayah, M.A.M. Gijs, Micromachining of glass inertial sensors, J. Microelectromechanical Syst. 11 (2002) 85-90.

[45] S. Schlautmann, H. Wensink, R. Schasfoort, M. Elwenspoek, A. van den Berg, Powderblasting technology as an alternative tool for microfabrication of capillary electrophoresis chips with integrated conductivity sensors, J. Micromechanics Microengineering. 11 (2001) 386-389.

[46] D.S. Park, T.I. Seo, M.W. Cho, Mechanical etching of micro pockets by powder blasting, Int. J. Adv. Manuf. Technol. 25 (2005) 1098-1104.

[47] C. Yamahata, F. Lacharme, Y. Burri, M. Gijs, A ball valve micropump in glass fabricated by powder blasting, Sens Actuators B. 110 (2005) 1-7. doi:10.1016/j.snb.2005.01.005.

[48] S. Masaki, S. Toshihiko, H. Kohei, I. Moriyasu, Gas-Particle Two-Phase Jet Flow from Slot Nozzle and Micro-Blasting Process, Trans. Jpn. Soc. Mech. Eng. B. 71 (2005) 2450-2457.

[49] T. Lomas, A. Wisitsoraat, F. Chevasuvit, A. Tuantranont, A precision hot embossing mold fabricated by high-resolution powder blasting with polydimethylsiloxane and SU-8 masking technology, J. Micromechanics Microengineering. 19 (2009) 35002.

[50] A. Ghobeity, H. Getu, T. Krajac, J.K. Spelt, M. Papini, Process repeatability in abrasive jet micro-machining, J. Mater. Process. Technol. 190 (2007) 51-60. doi:10.1016/j.jmatprotec.2007.03.111.

[51] D.B. Marshall, B.R. Lawn, A.G. Evans, Elastic/plastic indentation damage in ceramics: the lateral crack system, J. Am. Ceram. Soc. 65 (1982) 561-566.

[52] H.Z. Li, J. Wang, J.M. Fan, Analysis and modelling of particle velocities in micro-abrasive air jet, Int. J. Mach. Tools Manuf. 49 (2009) 850-858.

[53] M. Papini, D. Ciampini, T. Krajac, J.. Spelt, Computer modelling of interference effects in erosion testing: effect of plume shape, Wear. 255 (2003) 85-97. doi:10.1016/S00431648(03)00125-X.

[54] R.V. Wilson, A.O. Demuren, Numerical simulation of turbulent jets with rectangular crosssection, Inst. Comput. Appl. Sci. Eng. (1997).

[55] G. Kumi, C.O. Yanez, K.D. Belfield, J.T. Fourkas, High-speed multiphoton absorption polymerization: fabrication of microfluidic channels with arbitrary cross-sections and high aspect ratios, Lab. Chip. 10 (2010) 1057. doi:10.1039/b923377f.

[56] N. Futai, W. Gu, S. Takayama, Rapid Prototyping of Microstructures with Bell-Shaped Cross-Sections and Its Application to Deformation-Based Microfluidic Valves, Adv. Mater. 16 (2004) 1320-1323. doi:10.1002/adma.200400595. 
[57] J.R. Culham, Pressure Drop of Fully-Developed, Laminar Flow in Microchannels of Arbitrary Cross-Section, J. Fluids Eng. 128 (2006) 1037.

[58] E. Belloy, A.-G. Pawlowski, A. Sayah, M.A.M. Gijs, Microfabrication of high-aspect ratio and complex monolithic structures in glass, J. Microelectromechanical Syst. 11 (2002) 521527. doi:10.1109/JMEMS.2002.803418.

[59] H. Getu, J.K. Spelt, M. Papini, Thermal analysis of cryogenically assisted abrasive jet micromachining of PDMS, Int. J. Mach. Tools Manuf. 51 (2011) 721-730. doi:10.1016/j.ijmachtools.2011.05.003.

[60] Y. Kim, H. Lee, K. Lee, Y. Kim, Formation of Barrier Ribs for Plasma Display Panels via a Powder-Blasting Process: Part I, Effects of Binder Content and Baking Treatment, J. Am. Ceram. Soc. 87 (2004) 342-347. doi:10.1111/j.1551-2916.2004.00342.x.

[61] Y. Kim, H. Lee, K. Lee, Y. Kim, Formation of Barrier Ribs for Plasma Display Panels via Powder-Blasting Process: Part II, Effects of Powder-Blasting Angle, J. Am. Ceram. Soc. 87 (2004) 348-351. doi:10.1111/j.1551-2916.2004.00348.x.

[62] S. Ballandras, M. Wilm, M. Gijs, A. Sayah, E. Andrey, J.-J. Boy, L. Robert, J.-C. Baudouy, W. Daniau, V. Laude, Periodic arrays of transducers built using sand blasting and ultrasound micromachining techniques for the fabrication of piezocomposite materials, in: 2001: pp. 871-874 vol.2. doi:10.1109/ULTSYM.2001.991859.

[63] P.J. Slikkerveer, P.C.P. Bouten, F.C.M. De Haas, High quality mechanical etching of brittle materials by powder blasting, Sens. Actuators Phys. 85 (2000) 296-303.

[64] H. Getu, A. Ghobeity, J.K. Spelt, M. Papini, Abrasive jet micromachining of acrylic and polycarbonate polymers at oblique angles of attack, Wear. 265 (2008) 888-901. doi:10.1016/j.wear.2008.01.013.

[65] Y.I. Oka, H. Ohnogi, T. Hosokawa, M. Matsumura, The impact angle dependence of erosion damage caused by solid particle impact, Wear. 203-204 (1997) 573-579. doi:10.1016/S0043-1648(96)07430-3.

[66] T. Burzynski, M. Papini, Modelling of surface evolution in abrasive jet micro-machining including particle second strikes: A level set methodology, J. Mater. Process. Technol. 212 (2012) 1177-1190. doi:10.1016/j.jmatprotec.2012.01.002.

[67] G. Guan, L. Wu, A.A. Bhagat, Z. Li, P.C.Y. Chen, S. Chao, C.J. Ong, J. Han, Spiral microchannel with rectangular and trapezoidal cross-sections for size based particle separation, Sci. Rep. 3 (2013). doi:10.1038/srep01475.

[68] D. Sharma, P.P. Singh, H. Garg, Comparative Study of Rectangular and Trapezoidal Microchannels Using Water and Liquid Metal, Procedia Eng. 51 (2013) 791-796. doi:10.1016/j.proeng.2013.01.113.

[69] M. Akbari, D. Sinton, M. Bahrami, Viscous flow in variable cross-section microchannels of arbitrary shapes, Int. J. Heat Mass Transf. 54 (2011) 3970-3978. doi:10.1016/j.ijheatmasstransfer.2011.04.028.

[70] M. Bahrami, M.M. Yovanovich, J.R. Culham, Pressure Drop of Fully-Developed, Laminar Flow in Microchannels of Arbitrary Cross-Section, J. Fluids Eng. 128 (2006) 1036. doi:10.1115/1.2234786.

[71] Z. Duan, M.M. Yovanovich, Pressure drop for laminar flow in microchannels of arbitrary cross-sections, in: Semicond. Therm. Meas. Manag. Symp. 2009 SEMI-THERM 2009 25th Annu. IEEE, $\quad$ IEEE, 2009 : pp. 111-120. http://ieeexplore.ieee.org/xpls/abs_all.jsp?arnumber=4810751 (accessed May 4, 2016). 
[72] G. Croce, P. D'agaro, C. Nonino, Three-dimensional roughness effect on microchannel heat transfer and pressure drop, Int. J. Heat Mass Transf. 50 (2007) 5249-5259. doi:10.1016/j.ijheatmasstransfer.2007.06.021.

[73] V. Hessel, H. Löwe, F. Schönfeld, Micromixers - a review on passive and active mixing principles, Chem. Eng. Sci. 60 (2005) 2479-2501. doi:10.1016/j.ces.2004.11.033.

[74] F. Köhler, S. Schicho, B. Wolfrum, A. Gordijn, S.E. Pust, R. Carius, Gradient etching of silicon-based thin films for depth-resolved measurements: The example of Raman crystallinity, Thin Solid Films. 520 (2012) 2605-2608. doi:10.1016/j.tsf.2011.11.029.

[75] E. Belloy, A. Sayah, M.A.M. Gijs, Oblique powder blasting for three-dimensional micromachining of brittle materials, Sens. Actuators Phys. 92 (2001) 358-363.

[76] A. Ghobeity, M. Papini, J.K. Spelt, Abrasive jet micro-machining of planar areas and transitional slopes in glass using target oscillation, J. Mater. Process. Technol. 209 (2009) 5123-5132. doi:10.1016/j.jmatprotec.2009.02.012.

[77] N. Tamannaee, J.K. Spelt, M. Papini, Abrasive Slurry Jet Micro-machining of Edges, Planar Areas and Transitional Slopes in a Talc-Filled Co-Polymer, Precis. Eng. (2015). doi:10.1016/j.precisioneng.2015.06.009.

[78] M.C. Kong, S. Anwar, J. Billingham, D.A. Axinte, Mathematical modelling of abrasive waterjet footprints for arbitrarily moving jets: Part I-single straight paths, Int. J. Mach. Tools Manuf. 53 (2012) 58-68. doi:10.1016/j.ijmachtools.2011.09.010.

[79] J. Billingham, C.B. Miron, D.A. Axinte, M.C. Kong, Mathematical modelling of abrasive waterjet footprints for arbitrarily moving jets: Part II-Overlapped single and multiple straight paths, Int. J. Mach. Tools Manuf. 68 (2013) 30-39. doi:10.1016/j.ijmachtools.2013.01.003.

[80] S.M. Booij, Fluid jet polishing: possibilities and limitations of a new fabrication technique, [s.n.], 2003.

[81] H. Fang, P. Guo, J. Yu, Dwell function algorithm in fluid jet polishing, Appl. Opt. 45 (2006) 4291-4296.

[82] M.J. Vasile, J. Xie, R. Nassar, Depth control of focused ion-beam milling from a numerical model of the sputter process, J. Vac. Sci. Technol. B Microelectron. Nanometer Struct. 17 (1999) 3085. doi:10.1116/1.590959.

[83] A. Bilbao Guillerna, D. Axinte, J. Billingham, The linear inverse problem in energy beam processing with an application to abrasive waterjet machining, Int. J. Mach. Tools Manuf. 99 (2015) 34-42. doi:10.1016/j.jimachtools.2015.09.006.

[84] S.M. Booij, O.W. Fähnle, J.J. Braat, Shaping with fluid jet polishing by footprint optimization, Appl. Opt. 43 (2004) 67-69.

[85] A.D. Polianin, A.V. Manzhirov, Handbook of integral equations, 2nd ed, Chapman \& Hall/CRC, Boca Raton, 2008.

[86] I.I. Hirschman, D.V. Widder, The convolution transform, Courier Corporation, 2012.

[87] P. Rooney, A generalization of an inversion formula for the Gauss transformation, Cand Math Bull. 6 (1963) 45-53.

[88] D.R. Gossett, W.M. Weaver, A.J. Mach, S.C. Hur, H.T.K. Tse, W. Lee, H. Amini, D. Di Carlo, Label-free cell separation and sorting in microfluidic systems, Anal. Bioanal. Chem. 397 (2010) 3249-3267. doi:10.1007/s00216-010-3721-9.

[89] M.R. Sookhak Lari, M. Papini, Inverse methods to gradient etch three-dimensional features with prescribed topographies using abrasive jet micro-machining: Part I - Modeling, Precis. Eng. (2016). doi:10.1016/j.precisioneng.2016.03.004. 
[90] D. Di Carlo, Inertial microfluidics, Lab. Chip. 9 (2009) 3038. doi:10.1039/b912547g.

[91] E.S. Asmolov, The inertial lift on a spherical particle in a plane Poiseuille flow at large channel Reynolds number, J. Fluid Mech. 381 (1999) 63-87. doi:10.1017/S0022112098003474.

[92] J.-P. Matas, J.F. Morris, éLisabeth Guazzelli, Inertial migration of rigid spherical particles in Poiseuille flow, J. Fluid Mech. 515 (2004) 171-195. doi:10.1017/S0022112004000254.

[93] G.L. Morini, LAMINAR-TO-TURBULENT FLOW TRANSITION IN MICROCHANNELS, Microscale Thermophys. Eng. 8 (2004) 15-30. doi:10.1080/10893950490272902.

[94] A.G. Pawlowski, A. Sayah, M.A.M. Gijs, Precision poly-(dimethyl siloxane) masking technology for high-resolution powder blasting, Microelectromechanical Syst. J. Of. 14 (2005) 619-624.

[95] Y.S. Liao, L.C. Chen, Determination of mask opening size in creating a fluid hole on brittle material by double-side sand blasting, Int. J. Adv. Manuf. Technol. 29 (2006) 511-517. doi:10.1007/BF02729103.

[96] P.J. Slikkerveer, M.H.A. van Dongen, F.J. Touwslager, Erosion of elastomeric protective coatings, Wear. 236 (1999) 189-198.

[97] RapidMask HT UsersGuide II, (n.d.). http://www.ikonics.com/pdf/user-guide/ikonicsimaging/RapidMask_HT_UsersGuide_II.pdf.

[98] H. Wensink, J.W. Berenschot, H.V. Jansen, M.C. Elwenspoek, High resolution powder blast micromachining, in: 2000: pp. 769-774. doi:10.1109/MEMSYS.2000.838615.

[99] A. Grace, M. Works, Optimization Toolbox: For Use with MATLAB: User's Guide, Math works, 1990. 